City University of New York (CUNY) CUNY Academic Works

\title{
Novel Strategies For Glutamate Clearance in the Glia-Deprived Synaptic Hub of $C$. elegans
}

Joyce Chan

The Graduate Center, City University of New York

\section{How does access to this work benefit you? Let us know!}

More information about this work at: https://academicworks.cuny.edu/gc_etds/4627

Discover additional works at: https://academicworks.cuny.edu

This work is made publicly available by the City University of New York (CUNY).

Contact: AcademicWorks@cuny.edu 
NOVEL STRATEGIES FOR GLUTAMATE CLEARANCE IN THE GLIA-DEPRIVED SYNAPTIC HUB OF C. ELEGANS

by

JOYCE CHAN

A dissertation submitted to the Graduate Faculty in Biology in partial fulfillment of the requirements for the degree of Doctor of Philosophy, The City University of New York 
(C) 2021

\section{JOYCE CHAN}

All Rights Reserved 
Novel Strategies For Glutamate Clearance in the Glia-Deprived Synaptic Hub of C. elegans

$$
\text { by }
$$

Joyce Chan

This manuscript has been read and accepted for the Graduate Faculty in Biology in satisfaction of the dissertation requirement for the degree of Doctor of Philosophy

Date

Date
Jonathan Levitt

Chair of Examining Committee

Cathy Savage-Dunn

Executive Officer

Supervisory Committee:

Chris Li

Jeremy Dittman

Alexander Sobolevsky

THE CITY UNIVERSITY OF NEW YORK 


\begin{abstract}
Novel Strategies For Glutamate Clearance in the Glia-Deprived Synaptic Hub of C. elegans by Joyce Chan
\end{abstract}

Advisor: Itzhak Mano

As the major excitatory neurotransmitter in the mammalian brain, Glutamate (Glu) is critical for normal neuronal physiology. Disruption in Glu clearance results in hyper-stimulation of glutamatergic circuits, potentially leading to excitotoxic neurodegeneration. The canonical model of brain connectivity describes glutamatergic synapses as well insulated and enveloped by glia. These glia express Glu Transporters (GluTs) which work to clear Glu following synaptic activity. However, critical areas of the brain such as the mammalian hippocampus display poor synaptic isolation, which may result in Glu spillover between adjacent synapses and subsequent loss of circuit specificity. How accurate signal transmission is achieved in these glia-deprived areas remains unclear. We approach this question using the C. elegans system, which faces the same dilemma as the mammalian hippocampus. Using a combination of behavioral assays and imaging of transgenic animals expressing $\mathrm{Ca}^{2+}$-sensitive reporter GCaMP or Glu-sensitive reporter iGluSnFR, we demonstrate a combined role of synaptic location and pharyngeal pulsatility in efficient Glu clearance of the nerve ring. We find that perfusion of Glu-rich interstitial fluid plays a vital role in GluT-mediated synaptic clearance, shown in our experiments where we induce paralysis of pharyngeal and head muscles to inhibit local movement. This study provides novel insights to mechanisms of effective Glu clearance in the absence of glia, a feature shared between nematodes and vital areas of the mammalian brain. Investigating novel mechanisms of Glu clearance can inform us in seeking candidate targets for therapeutic interventions in brain ischemia, and potential ways of manipulating brain pulsations and heart rate to promote clearance of solutes and aggregates (such as amyloid $\beta$ ) in patients to slow the onset of Alzheimer's disease and other neuropathies. 


\section{Acknowledgements}

My work over the course 5 years in Mano Lab would not have been possible without the support of my beloved mentors, colleagues, friends, and family. I dedicate this work to my family in hopes that I make them proud and to honor their efforts in providing their children the best possible future. My project has been funded by NIH under grant number 5R21NS098350-02.

I also want to thank my colleagues, both past and present, from the Mano lab. I thank Itzik for providing me this opportunity to work on this fascinating project that have driven my curiosity for the past 5 years. Genevieve Feldmann and Ayesha Chowdhury provided instruction early on and helped me acclimate to working as a doctoral student in the lab environment. Zelda Mendelowitz, who has been in Mano lab with me since the very beginning and through discussions have provided me much insight and clarity. Adem Idrizi believed that I could microinject (and was also capable of so much more!), and gave me the push that I needed before pandemic struck and I had to stand on my own. I also thank Kyungwha Lee, who I share first coauthorship of my first manuscript with, for paving the road with experiments that were the foundation for the AVA project. Itzik and Irving provided valuable input and expertise in techniques in advancing my projects, and I thank also the undergrads for giving me the opportunity to teach them and for the lessons that they taught me in return.

I would also like to thank my mentors who have accompanied me at CDI and continually push me to become a stronger mentor, a better person, and a supportive friend until the end. My first CCNY Women in Science mentor, Sruti Patoori, who has helped me navigate through undergrad and then the exciting journey through graduate school, and who I have shared countless many adventures with that I would not trade for anything else in the world. Brian Wey, for always being frank, supportive, and endlessly talented, in addition to humoring my nerdy, non-science interests. Adanna Alexander, for being the best listener that I know of, and for helping me find my ground when I needed it the most. I also express gratitude towards Shirley Chan; I don't know how it would've been possible to survive that first year back in lab during a pandemic amidst all the uncertainty without her, and realizing I still have much to learn about myself. You all have helped me so much in making sense of navigating academia and the spaces outside of it.

I also express thanks to Dr. Shubha Govind and Dr. Ana Carnaval, who took me on as an undergraduate research student and continually inspired and supported me, even after I moved on from working in their labs. I also thank our Dean of Sciences, Dr. Susan Perkins, for her tireless efforts in ensuring that students learn and researchers work and that everything runs as smoothly and safely as possible. Even the smallest of actions and kindest of words helped to immensely boost morale for me and other students who have been fortunate enough to work with them, and I aspire to have the same strength to support and inspire the next generation of students and researchers.

I want to thank my committee members: Dr. Chris Li, Dr. Jonathan Levitt, Dr. Jeremy Dittman, and Dr. Alexander Sobolevsky. All the members provided helpful advice, feedback, and their expertise that drove my research to success. I thank the Li and Emerson labs for being wonderful 
lab neighbors, whether it is for borrowing a reagent or hearing their experiences with different techniques.

I also want thank the GAP Alliance, APALA, the enormous and impactful activists driving the BLM movement, and 18 Million Rising for helping me make sense of the social patterns and histories that have shaped our world. I thank CCNY Women in Science for providing an space where we can work towards the advancement of women and minoritized scientists. To quote from the second Eurasian Women's Forum: "The world need science, and science needs women". I also want to acknowledge the noble contributions of doctors and scientists worldwide that have worked hard to ensure our survival during the pandemic. I hope that one day the full scope of their contributions will be properly rewarded and recognized in a future where our society places greater value on empathy and science rather than fear and judgement clouded by misinformation and personal prejudices.

Thank you all for believing in me, and trusting that I can overcome the obstacles that came with pursuing my doctorate's degree. This truly would have been an impossible journey without you all! 


\section{Table of Contents}

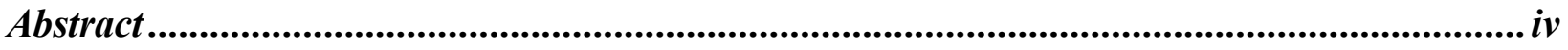

Acknowledgements...........................................................................................................v

Table of Contents....................................................................................................... vii

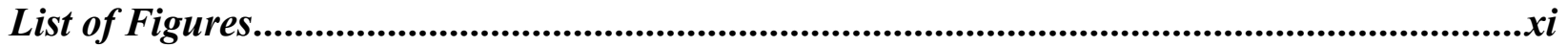

Chapter 1: Introduction and Background..........................................................................1

1.1.1. Neuronal processing depends on circuits insulation, Glu clearance, and avoiding

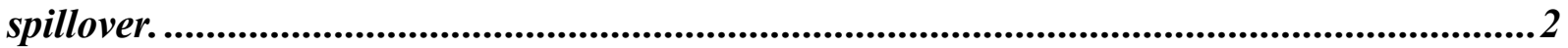

1.1.2. The choice of Glutamate as a major excitatory neurotransmitter is unusual. ..............3

1.1.3. Critical brain areas defy the canonical model of Glu clearance................................3

1.1.4. Extracellular space takes up a larger portion of brain volume than previously

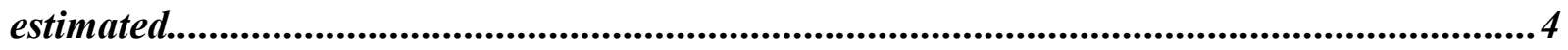

1.1.5. The physiology and structure of Glu Transporters (GluTs) enable robust clearance...5

1.1.6. Caenorhabditis elegans is an advantageous model for studying GluT activities. ........ 7

1.1.7. The C. elegans nerve ring is a dense synaptic hub deprived of glial insulation............ 7

1.1.8. Nematode GluTs can be divided into Proximal and Distal classes ............................8

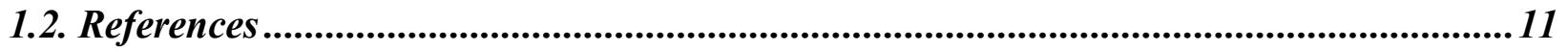


Chapter 2: Novel Strategies for Glutamate Clearance in the Glia-Deprived Synaptic Hub of C.

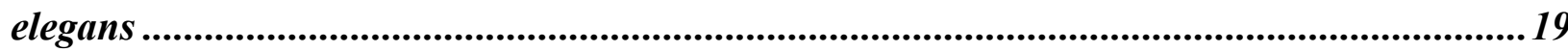

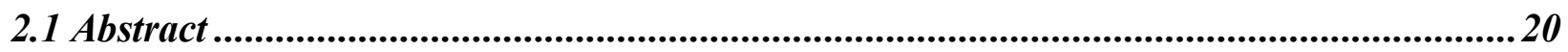

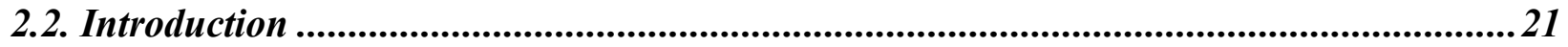

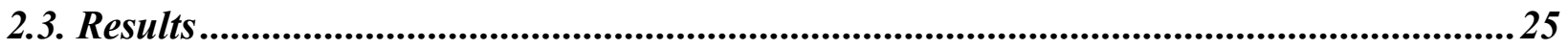

2.3.1. Behaviors mediated by the avoidance circuit are strongly sensitive to manipulation of

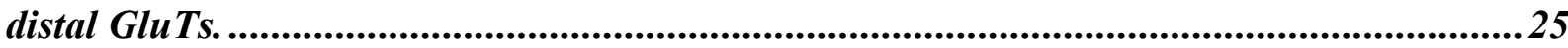

2.3.2. Hyperstimulation of the avoidance circuit in distal GluT KO animals is evident in calcium and glutamate reporter recordings.........................................................................26

2.3.3. Reduction of proximal Glu clearance generates avoidance response to low concentration of $\mathrm{NaCl}$.

2.3.4. Reduction of proximal Glu clearance causes abnormal AVA activation in response to low $\mathrm{NaCl}$ concentration increase. ...............................................................................34

2.3.5. Proximal and distal GluTs are at least partially non-interchangeable, suggesting they are endowed with distinct functional properties. .............................................................41

2.3.6. Differential localization of ASH and ASE synapses in the nerve ring might contribute to the differential effect of proximal and distal Glu clearance.............................................46

2.4. Discussion and Conclusion .............................................................................56

2.5. Materials and Methods........................................................................................ 62

2.6. Acknowledgements ............................................................................................... 70

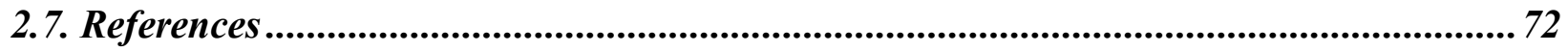


Chapter 3: Synaptic maintenance and differentiation of signals within a neuron where Glu is both an excitatory and inhibitory signal.

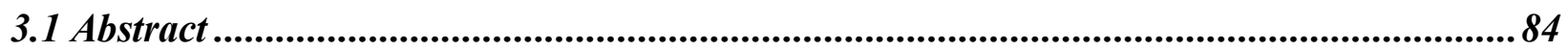

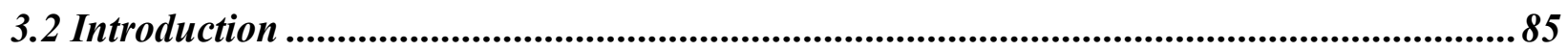

3.2.1. Glu signaling in the ASE->AIB system salt chemotaxis circuit is asymmetrical and highly complex. .............................................................................................................. 85

3.2.2. Location of ASER->AIB and ASEL->AIB and differential localization of GluRs may be a determinant of how Glu signals are interpreted by AIB. .........................................8 87

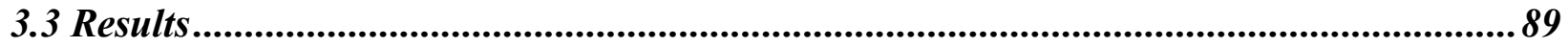

3.3.1. GluT function is needed to mediate effective clearance of ASE->AIB synapses. .......89

3.3.2. Motility of the head and pharynx is critical to preserving the fidelity of AIB synaptic

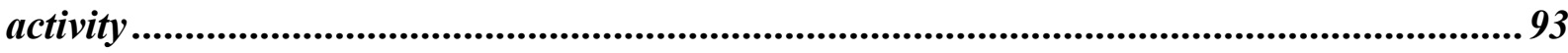

3.4 Discussion and Conclusion .................................................................................................. 110

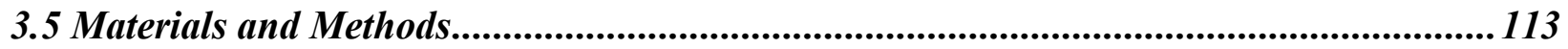

3.6 Acknowledgements.............................................................................................. 118

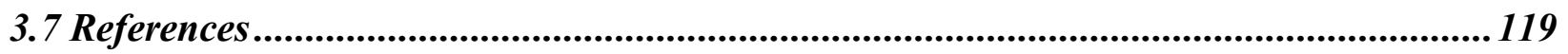

Chapter 4: Conclusions, Discussions, and Further Experiments ......................................... 124

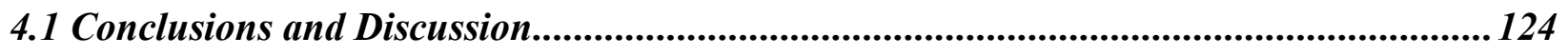

4.2 Future Experiments .................................................................................................. 129

4.2.1. Analyzing GluT properties through heterologous expression in Xenopus oocytes. .. 129 
4.2.2. Determining how AIB neurons distinguishes between excitatory and inhibitory signals when Glu serves as the neurotransmitter for both responses.

4.2.3. Tracking diffusion and localization of Glu released from different points of origin.

4.2.4. Investigating where GLT-4 acts pre-synaptically as a chloride-conducting GluT to negatively regulate Glu release.

4.4.1. Members of the mammalian Excitatory Amino Acid Transporter (EAAT) family... 138

4.4.2. Strains used in behavioral assays and imaging experiments 


\section{List of Figures}

1. Transport dynamics of GluTs 5

2. Sequence alignment of C. elegans GluTs reveal unique differences in core binding domains 6

3. Glu is the major excitatory neurotransmitter used in C. elegans 8

4. Proximal and distal GluTs in C. elegans 9

5. Predictive modeling of $C$. elegans GluTs reveals high structural similarities with bacterial GltPh 23

6. Distal GluT KOs disrupt behaviors mediated by the ASH $->$ AVA avoidance circuit 25

7. Distal GluT KO causes exaggerated response of AVA to ASH stimulation, and is accompanied by increase of Glu signals on AVA neurites

8. ASH is the source of Glu signals that directly result in the exaggerated AVA responses seen in Distal GluT KO animals in response to stimulation by $1 \mathrm{M}$ glycerol

9. ASH neurons respond only to high concentration $\mathrm{NaCl}$

10. Absence of proximal GluTs abnormally switches worms response to low concentration $\mathrm{NaCl}$ from attraction to avoidance

11. Proximal GluT KOs cause AVA to respond to ASEL stimulation, accompanied by Glu signal on the AVA neurites

12. AVA spillover responses to $50 \mathrm{mM} \mathrm{NaCl}$ increase are also affected by proximal GluT $\mathrm{KO}$ and depend on Glu release from ASEL (similar to responses to $1 \mathrm{mM} \mathrm{NaCl}$ increase)

13. A $1 \mathrm{mM} \mathrm{NaCl}$ decrease does not stimulate AVA neurons of GluT KO animals 
14. The effect of proximal GluT KO on AVA response to ASEL stimulation is mediated by spillover of Glu released from ASEL

15. ASEL-specific eat-4 rescues respond to neither $1 \mathrm{mM}$ decrease in $\mathrm{NaCl}$ concentration nor $1 \mathrm{M}$ glycerol stimulation

16. ASH-specific rescue of Glu release in proximal GluT KO animals in a mutant eat-4 background fails to restore putative spillover onto AVA in response to $1 \mathrm{mM} \mathrm{NaCl}$ stimulation

17. Distal and proximal GluTs are partially distinct in their molecular function.

18. glt-1 mutant animals overexpressing GluTs do not display exaggerated AVA responses to ASH stimulation, nor do glt-3 mutant animals display aberrant AVA responses to ASEL stimulation

19. Analysis of EM data from the C. elegans nerve ring suggests that synapses of different circuits are found in great proximity

20. Analysis of EM data from the C. elegans nerve ring suggests that synapses of different circuits are found in great proximity

21. ASH responds to $1 \mathrm{M}$ glycerol before and during paralysis with $2 \mathrm{mM}$ tetramisole

22. Sequential stimulation of worms with $1 \mathrm{M}$ glycerol or $1 \mathrm{mM} \mathrm{NaCl}$ does diminish not exaggerated responses in AVA or putative spillover from ASE

23. Paralysis eliminates or masks the exaggerated and ectopic postsynaptic responses of AVA

24. BDM-induced paralysis eliminates exaggerated responses in the ASH -> AVA synapse to $1 \mathrm{M}$ glycerol 
26. The two-tier model of Glu clearance in C. elegans

27. Visualization of ASE and AIB synaptic connections

28. GluT KO mutants display heightened Glu-mediated responses of AIB to stimulation of sensory neuron ASER by a $50 \mathrm{mM} \mathrm{NaCl}$ downstep

29. GluT KO mutants display prolonged and more intense Glu-mediated inhibition of AIB to stimulation of sensory neuron ASEL by a $50 \mathrm{mM} \mathrm{NaCl}$ upstep 90

30. Paralysis eliminates excitatory AIB responses to $50 \mathrm{mM} \mathrm{NaCl}$ downstep

31. Paralysis eliminates AIB inhibition response to $50 \mathrm{mM} \mathrm{NaCl}$ upstep

32. Paralysis eliminates excitatory AIB responses to $1 \mathrm{mM} \mathrm{NaCl}$ downstep

33. Paralysis eliminates $\mathrm{AIB}$ inhibition responses to $1 \mathrm{mM} \mathrm{NaCl}$ upstep 98

34. Specific markers allow for distinction between proximal and distal AIB neurites 99

35. GluT KO mutants do not display significantly different $\mathrm{Ca}^{2+}$ responses in proximal AIB neurites to an excitatory $50 \mathrm{mM} \mathrm{NaCl}$ downstep

36. GluT KO mutants do not display significantly different $\mathrm{Ca}^{2+}$ responses in distal AIB neurites to an excitatory $50 \mathrm{mM} \mathrm{NaCl}$ downstep

37. GluT KO mutants do not display significantly different $\mathrm{Ca}^{2+}$ responses in proximal AIB neurites to an inhibitory $50 \mathrm{mM} \mathrm{NaCl}$ upstep

38. GluT KO mutants do not display significantly different $\mathrm{Ca}^{2+}$ responses in distal AIB neurites to an inhibitory $50 \mathrm{mM} \mathrm{NaCl}$ upstep

39. Alignment of $C$. elegans GluT domain TM2 with hEAAT5

40. Predictive modeling of $C$. elegans GluTs reveals high structural similarities with bacterial Glt

41. Differences in orientation of conserved residues can potentially affect $\mathrm{Na}^{+}$ion affinity 
42. Differences in orientation of conserved residues can potentially affect ligand affinity

43. Schematic for distinguishing between proximal and distal neurite regions for AIB-

133 specific iGluSnFR 


\title{
Chapter 1: Introduction and
}

\author{
Background
}




\subsubsection{Neuronal processing depends on circuits insulation, Glu clearance, and avoiding}

\section{spillover.}

In the mammalian brain, accurate neurotransmission between members of neuronal circuits is essential in forming specific cell assemblies that encode information and mediate behavioral output in response to distinct stimuli and environmental conditions ${ }^{1,2,3,4}$. Synaptic (wired) communication requires accurate synaptic transmission and neuronal processing, depending on clear distinction of synapses and circuits by insulation and robust clearance, warding off "spillover", the spread of neurotransmitter between adjacent synapses ${ }^{5,6}$. Glia cells that provide anatomical separation of synapses in the central nervous system (CNS) express neurotransmitter transporters that function to sequester and maintain low levels of chemical signals at the synapse 7,8. Loss of transporter function can result in neuronal hyperactivity and spillover between circuits, leading to behavioral defects and, in extreme cases, excitotoxic neurodegeneration ${ }^{9,10,11}$. However, it should also be noted that spillover is a vital component in configurations that utilize volume transmission (not only for localized Glu signaling in the hippocampus, but also for normal dopamine transmission in the striatum $)^{6,12}$. Glu spillover is also needed in areas such as basal dendrites of cortical pyramidal neurons, where activation of extrasynaptic NMDA receptors at dendrites and nearby spines generates NMDA spikes, even in the absence of AMPA receptor activation ${ }^{13}$. More recent studies on long-term potentiation (LTP) have also unveiled a complex relationship where spillover and synaptic cross-talk between neighboring synapses negatively regulates glial coverage, a form of synaptic remodeling observed when perisynaptic astroglia withdraw their processes over time in response to the heightened frequency of signal spillover triggered during $\mathrm{LTP}^{14,15}$. Much remains to be understood in synaptic organization, diffusion, and diversity of transporter properties. 


\subsubsection{The choice of Glutamate as a major excitatory neurotransmitter is unusual.}

Glutamate (Glu) is the primary fast excitatory neurotransmitter of the nervous system, serving roles in learning and memory, long-term potentiation, and synaptic plasticity ${ }^{3,16,17,37}$. Despite its widespread use in the vertebrate CNS, Glu has several characteristics that make synaptic regulation more difficult compared to neurotransmitters such as acetylcholine (ACh), which has wide use in most invertebrates. Unlike Ach, Glu does not require a unique neurotransmitter synthesis pathway, and lacks enzymatic inactivation of Glu excesses at the synapse ${ }^{18}$. Instead, clearance depends on expression of cell surface Glu Transporters (GluTs) to move Glu into glia cells. Thus, previously released Glu is sequestered from synaptic Glu Receptors (GluRs), and can be recycled through the glutamate-glutamine cycle ${ }^{19-21}$. However, this arrangement makes the system highly sensitive to malfunction or absence of these GluTs, leading to incomplete Glu clearance of synapses. This increases the potential of spillover, especially when the majority of neurons and synapses are glutamatergic, resulting in over-excitation and blurred circuit precision $^{22}$. Glu accumulation at high enough levels can lead to excitotoxic neurodegeneration as excess amounts of $\mathrm{Ca}^{2+}$ enter neurons through $\mathrm{GluRs}^{9,23,24}$.

\subsubsection{Critical brain areas defy the canonical model of Glu clearance.}

The canonical model of brain connectivity describes Glu synapses as well insulated and enveloped by glia ${ }^{25,26}$. While high coverage of perisynaptic glia are seen in some parts of the CNS, other critical regions of the mammalian brain (e.g., hippocampus) show low glial coverage and a high percentage of uninsulated synapses $(\sim 66 \%)^{24,25}$. This arrangement is surprising, since the mammalian hippocampus is vital to regulating learning and memory ${ }^{1,27}$. The open architecture of synapses in these areas suggest that other means such as rapid diffusion ${ }^{28,29-32}$ 
may contribute to reducing the effect of spillover in these areas. The concept of glial GluTs dominating Glu clearance falls short of explaining how signaling fidelity is maintained in these glia-poor brain regions. Consequently, it is worthwhile to re-evaluate the contribution of diffusion of extracellular space (ECS) to Glu clearance.

\subsubsection{Extracellular space takes up a larger portion of brain volume than previously estimated.}

Traditional EM studies of the mammalian brain depict the neuronal parenchyma as dense, with only a small fraction of volume occupied by ECS/interstitial fluid. However, biophysical measurements suggest much larger ECS volumes ${ }^{31}$. Recent findings reveal that chemical treatments used in EM cause glial swelling and overall shrinkage of samples, significantly underrepresenting ECS volumes ${ }^{52,5333}$. This notion is further supported by super-resolution imaging of live brain tissue, showing that in layers rich in synapses (as opposed to cell bodies), the ECS fraction climbs to $20-30 \%{ }^{33,34,35}$. This new validation of the abundance of ECS/interstitial fluid in the mammalian CNS coincides with the recent recognition of the glymphatic system of the brain, where para-vascular space around capillaries and larger vessels contain cerebrospinal fluid (CSF). The flow of CSF through the interstitial fluids of the brain parenchyma to drainage sites has important implications in neurological disease and behavior, facilitating clearance of macromolecules such as $A \beta^{36-43}$. This clearance is further aided by vascular pulsatility ${ }^{38,44,45}$. Altogether, these notions suggest that Glu clearance from the immediate vicinity of synapses in these specific areas of the brain may be partly mediated by convection/bulk flow (i.e., transport by hydrodynamic flow) $)^{31,32,46}$, a larger-scale process that follows the rapid diffusion of Glu from the synapse. Whether immediately perisynaptic or at a more distal location, Glu must be eliminated from ECS/interstitial fluids by specialized cell surface transporters. 


\subsubsection{The physiology and structure of Glu Transporters (GluTs) enable robust clearance.}

GluTs are cell-surface, high-affinity, $\mathrm{Na}^{+}$-dependent transporters ${ }^{7,34,47-50}$, playing key roles in CNS physiology and prevention of pathological conditions ${ }^{11,51}$. The GluT family consists of 5 mammalian members, with glial GLT-1/EAAT2 responsible for $90 \%$ of all brain Glu clearance (see Table 1). GluT function depends on the ion gradients set by the $\mathrm{Na}^{+} / \mathrm{K}^{+}$-ATPase ${ }^{22}$. GluTs cotransport $3 \mathrm{Na}^{+}$and a $\mathrm{H}^{+}$ion coupled with a Glu (or Asp) and the counter-transport of a $\mathrm{K}^{+}$ion

(Figure 1a). This transport is electrogenic, with the coupling of ions generating a powerful driving force. This causes flux reversal at resting $\mathrm{V}_{\mathrm{m}}$, maintaining Glu levels in the nanomolar range (Figure 1b). A number of GluT are also capable of conducting $\mathrm{Cl}^{-}$, but this conductance is uncoupled from Glu transport and occurs by diffusion through an anion-channeling pathway ${ }^{22,52}$.

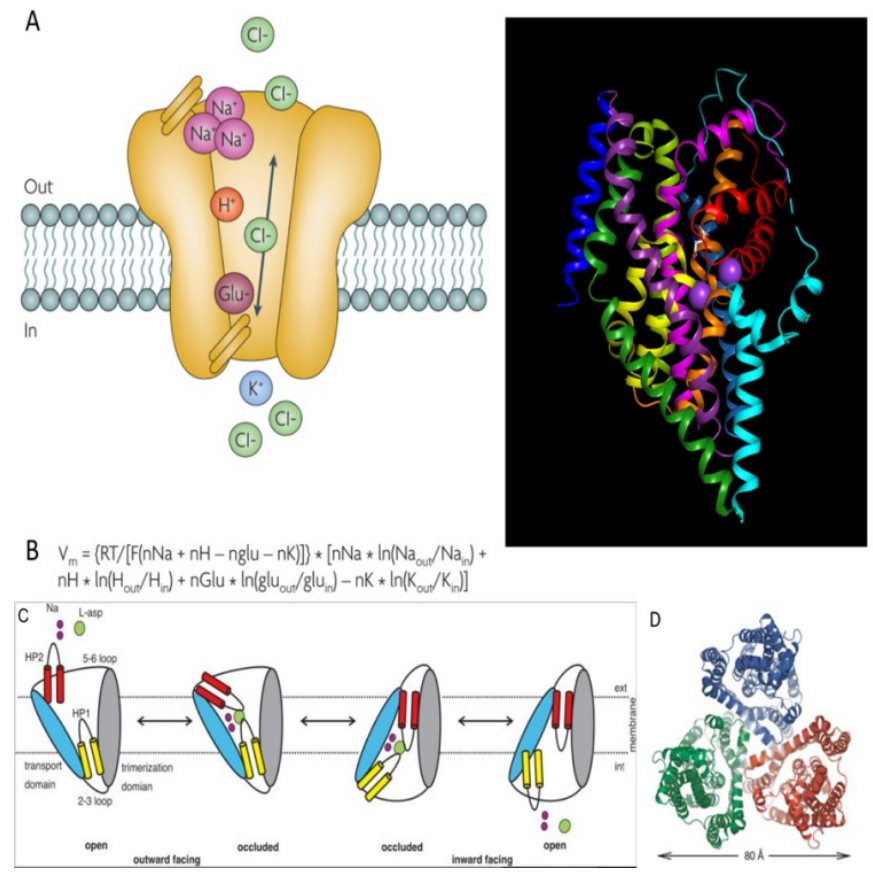
Figure 1: Transport dynamics of GluTs. (A) Left: Transport stoichiometry in GluTs, involving co- transport of Glu with 3 sodiums and a proton and counter-transport of potassium. Some GluTs also show uncoupled flux of $\mathrm{Cl}^{-}$. Right: Model of a GltPh subunit in the outward facing state, with bound Aspartate (white) and $2 \mathrm{Na}^{+}$(purple spheres). The core binding domains are color-coded the following: HP1 in yellow, TM7 in orange, HP2 in red, and TM8 in magenta. (B) Goldman zero-flux equation predicting the individual permeabilities of ions and Glu at both sides of the membrane during equilibrium. Modified from Tzingounis and Wadiche, 2014. (C) Schematic of outward facing and inward facing conformations of GltPh. Grey region indicates the immobile trimerization domain, while blue denotes the mobile transport domain. Schematic acquired from Reyes et al., 2009. (D) Ribbon representation of GltPh fully assembled as a homotrimer, viewed from the extracellular side of the membrane. Modified from Yernool et al., 2004.

$\mathrm{Cl}^{-}$conductance differs between family members, and has the greatest consequence in presynaptically expressed GluTs of the retina (EAAT5) ${ }^{22}$. Initial crystallography of a bacterial GluT homolog from Pyrococcus horikoshii revealed the topology of GluTs to consist of eight transmembrane (TM) and two hairpin loop (HP) domains ${ }^{53,54}$. GluTs form a homotrimeric 
complex with independently-functioning subunits $22,53,55$. The substrate-binding core domain consist of specific residues located on HP1, TM7, HP2, and TM8 ${ }^{16,56,56,57}$. While TM1-TM6 form a stable scaffold, the core domain forms an elevator structure that shuttles the substrates in and out of cells. A notable feature highly conserved across GluT subtypes and species is a 'NMDGT' motif in TM7 (Figure 2), which has been shown to interact with Glu and $\mathrm{Na}^{+}$. Both $\mathrm{GltPh}$ and human Excitatory Amino Acid Transporter 1 (EAAT1) have been shown to bind Aspartate (Asp), which has structural similarity to Glu. Although GltPh solely binds Asp and not Glu, EAAT1 has a stronger Glu preference ${ }^{55,56}$. Importantly, Asp/Glu affinity is dependent on $\mathrm{Na}^{+}$ concentration; $\mathrm{Na}^{+}$binding induces a conformational change in the binding domain to become more conducive to binding Asp/Glü5.

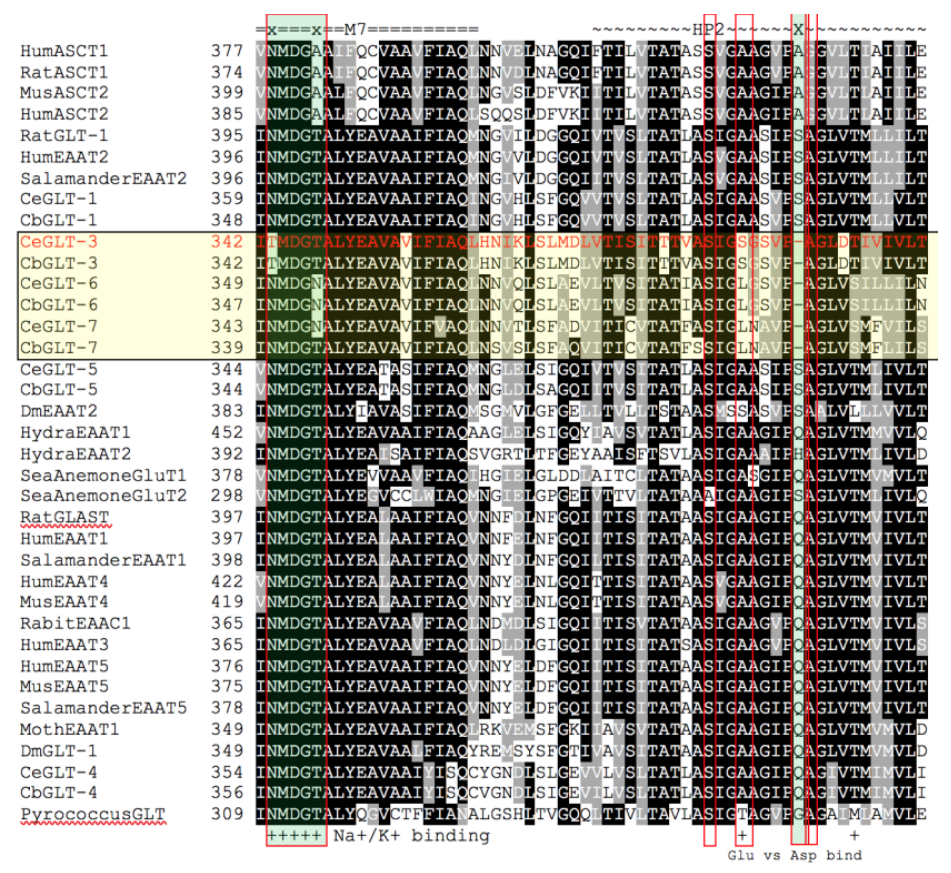

Figure 2. Sequence alignment of $C$. elegans GluTs reveal unique differences in core binding domains. Unique differences have been identified in nematode Distal GluT sequences, particularly those that have the potential to affect substrate binding dynamics. These residues include those involved in binding of $\mathrm{Na}^{+} / \mathrm{K}^{+}$ions, Glu / Asp binding, or $\mathrm{Cl}^{-}$ channel gating.

While great progress has been made in recent years in understanding synaptic anatomy and the biophysical properties of GluTs, a full schema of how anatomical organization and clearance physiology work in tandem to maintain circuit specificity remains incomplete. In particular, mammalian studies are hindered by the fact that most studies are performed ex-vivo, 
or require intrusive operations that interfere with fluid physiology. It is therefore a high priority to study systemic Glu clearance in an intact animal model that allows for accessibility and precise monitoring of activity in specific synapses and circuits.

\subsubsection{Caenorhabditis elegans is an advantageous model for studying GluT activities.}

To elucidate these clearance strategies in intact animals, we use the nematode Caenorhabditis elegans as a model. The nematode bypasses the major limitations of traditional mammalian models used in neuroscience studies; its transparency allows real-time optogenetic imaging of any neuron in vivo, without the use of intrusive procedures. Additionally, all neuronal circuits are well characterized, from individual neurons and synapses to their functional behavioral output ${ }^{58}$. In contrast, the full human and mouse connectome is overly complex and has yet to be mapped. The molecular building blocks of Glu signaling are well conserved; nematode GluRs and GluTs are highly homologous to their mammalian counterparts in sequence and function, as Glu is a major excitatory neurotransmitter in central synapses ${ }^{53,59}$. As such, the C. elegans system is ideal for our studies.

\subsubsection{The C. elegans nerve ring is a dense synaptic hub deprived of glial insulation.}

Out of the nematode's total 302 neurons, 78 release Glu as a neurotransmitter, and 118 express GluRs (Figure 3) ${ }^{60,61}$. Many glutamatergic neurons are located in the head ganglion, forming synaptic connections at the nerve ring the nematode's largest synaptic hub. It is situated between the two bulbs of the pharynx, with the inner rim facing the psuedocoelomic space separating it from the pharynx, a region directly exposed to body fluids churned by pharyngeal pulsations ${ }^{58}$. Only four glial cells (the cephalic sheath glia) enwrap the nerve ring from the outside, but do not 
physically separate individual synapses ${ }^{58}$.

A

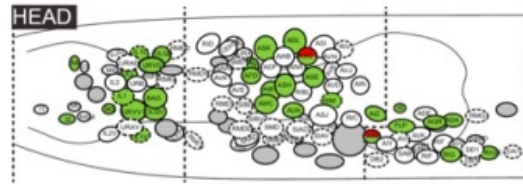

78/302 neurons release Glu as a NT

B

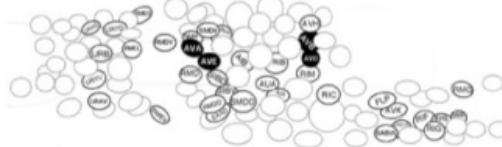

118/302 express postsynaptic excitatory Glutamate Receptors (GluRs)

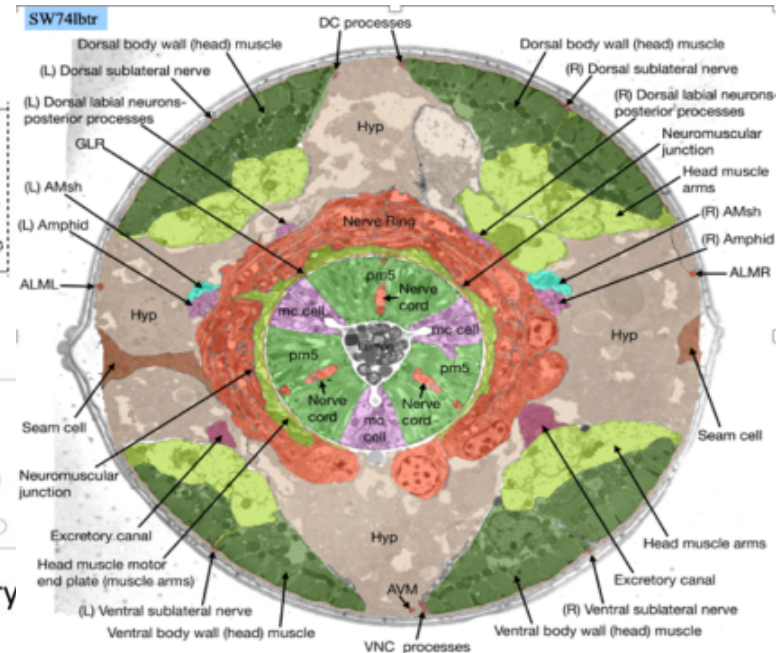
Figure 3: Glu is the major excitatory neurotransmitter used in the C. elegans. (A) The C. elegans head
ganglia, glutamatergic neurons shaded in green. (B) GluR-expressing neurons of the head ganglia. Each labeled neuron expresses at least one of 6 nematode GluR subunits. Neurons filled in black are interneurons expressing many GluRs. (C) Transverse cross-section of the nematode head region, displaying location of the nerve ring (red) relative to the pharynx, hypodermis and head muscles. Adapted from WormAtlas (Altun, 2018).

\subsubsection{Nematode GluTs can be divided into Proximal and Distal classes}

Our lab has previously found that the six C. elegans GluTs are expressed in distinctive patterns.

Proximal GluTs primarily expressed in the nematode head region are: GLT-1, which is expressed in the body wall muscles of the head and hypodermis, and glia ${ }^{62,63}$; and GLT-4, which has been found on some pre-synaptic neurons (Figure 4) ${ }^{62}$. Intriguingly, we found that three GluTs (GLT3, GLT-6, and GLT-7) are expressed on the capillary canal cell spanning the length of the body (at least $7 \mu \mathrm{m}$ ), and act distally from the glutamatergic synapses in nerve ring to affect efficient clearance. Nonetheless, $\mathrm{KO}$ of these distal GluTs have specific effects on known Glu-mediated behaviors, consistent with the effects of GluT KO on raising synaptic Glu levels. The role of distal GluT in synapse physiology is further emphasized by the observation that, in a sensitized background, $\mathrm{KO}$ of distal (but not proximal) GluTs causes excitotoxic neurodegeneration ${ }^{64}$, despite exhibiting few major deviations from consensus sequences in the core binding area 
(Figure 2). These differences are expected to modify substrate interactions, suggesting that distal GluTs have unique functional properties.

The unusual glia-deprived organization of synapses in the nerve ring and the existence of distal GluTs prompted us to study the activities of different GluT in depth, and evaluate the
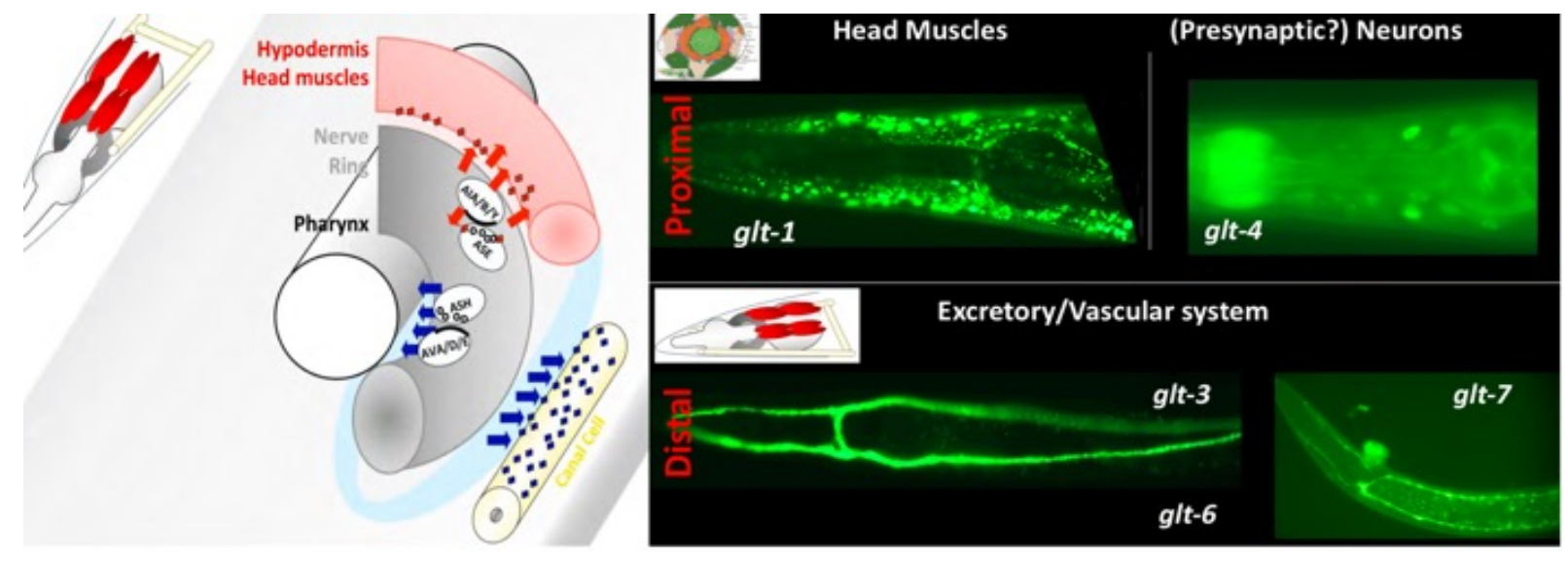

Figure 4: Proximal and distal GluTs in C. elegans. (Left) Model of Glu clearance in the C. elegans head. Synapses facing the inner rim of the nerve ring (e.g. ASH->AVA/AVD/AVE) are preferentially cleared by distal GluTs, whereas synapses on the outer rim (e.g ASE-> AIA/AIB/AIY) are preferentially cleared by proximal GluTs. (Right) Expression of $C$. elegans GluT using GFP fusion proteins. glt- 1 is expressed in head muscles and hypodermis, glt-4 is expressed in some pre-synaptic head neurons, and glt-3, along with $g l t-6$ and $g l t-7$, are expressed on canal cells.

effect of GluT KOs in modifying behaviors mediated by glutamatergic synapses. In this study, we ask if the challenges of a dense synaptic hub are addressed in C. elegans by a complimentary Glu clearance system, whereby the lack of anatomical synaptic barriers is compensated by clearance-mediated physiological separation to maintain the accuracy of Glu signaling and circuit resolution. We hypothesize that GluTs display differential activity over specific synapses, based on arrangement of synaptic location and dependence on flow of extracellular fluids (Figure 4). The concept of perfusion-mediated clearance in nematodes is also in line with the anatomical observation that nematode synapses are aspiny (in contrast to mammalian neurons that are rich in dendritic spines), and formed en passant ${ }^{65-67}$, promoting perisynaptic diffusion. With my data, I demonstrate that a glutamatergic circuit located on the inner rim of the nerve 
ring relies heavily on distal GluT activity for synaptic clearance. I further demonstrate that an adjacent glutamatergic circuit located on the outer rim of the nerve ring relies on the combined efforts of proximal and distal GluTs in maintain low synaptic Glu levels. Lastly, I show evidence that successful clearance of both inner rim and outer rim synapses requires mobility of the head and pharynx.

Our overall hypothesis is that Glu clearance from synapses of aspiny neurons in absence of glial insulation relies on particularly robust clearance, combining an array of GluTs and bulk flow. GluTs differing in expression pattern and function (the proximal and distal GluTs) must work in concert to prevent Glu over-accumulation and intersynaptic spillover, safe-guarding neurons against excitotoxicity. 


\subsection{References}

1. Neves, G., Cooke, S. F. \& Bliss, T. V. P. Synaptic plasticity, memory and the hippocampus: a neural network approach to causality. Nature Reviews Neuroscience 9, 65-75 (2008).

2. Rogerson, T. et al. Synaptic tagging during memory allocation. Nature Reviews Neuroscience 15, 157-169 (2014).

3. Yoshio, S. How do cell assemblies encode information in the brain? Neuroscience \& Biobehavioral Reviews 23, 785-796 (1999).

4. Buzsáki, G. Neural Syntax: Cell Assemblies, Synapsembles, and Readers. Neuron 68, $362-385$ (2010).

5. Huang, Y. H. \& Bergles, D. E. Glutamate transporters bring competition to the synapse. Current Opinion in Neurobiology 14, 346-352 (2004).

6. Taber, K. H. \& Hurley, R. A. Volume Transmission in the Brain: Beyond the Synapse. $J N P$ 26, iv-4 (2014).

7. Danbolt, N. C. Glutamate uptake. Progress in Neurobiology 65, 1-105 (2001).

8. Herman, M. A. \& Jahr, C. E. Extracellular Glutamate Concentration in Hippocampal Slice. Journal of Neuroscience 27, 9736-9741 (2007).

9. Fricker, M., Tolkovsky, A. M., Borutaite, V., Coleman, M. \& Brown, G. C. Neuronal Cell Death. Physiological Reviews 98, 813-880 (2018).

10. Choi, D. W. Excitotoxic cell death. Journal of Neurobiology (1992) doi:10.1002/neu.480230915. 
11. Rothstein, J. D. et al. Knockout of Glutamate Transporters Reveals a Major Role for Astroglial Transport in Excitotoxicity and Clearance of Glutamate. Neuron 16, 675-686 (1996).

12. Wiencke, K., Horstmann, A., Mathar, D., Villringer, A. \& Neumann, J. Dopamine release, diffusion and uptake: A computational model for synaptic and volume transmission. PLoS Comput Biol 16, e1008410 (2020).

13. Chalifoux, J. R. \& Carter, A. G. Glutamate Spillover Promotes the Generation of NMDA Spikes. Journal of Neuroscience 31, 16435-16446 (2011).

14. Henneberger, C. et al. Astroglia withdraw from potentiated synapses boosting intersynaptic cross-talk. (2018) doi:10.1101/349233.

15. Henneberger, C. et al. LTP Induction Boosts Glutamate Spillover by Driving Withdrawal of Perisynaptic Astroglia. Neuron 108, 919-936.e11 (2020).

16. Attwell, D. \& Gibb, A. Neuroenergetics and the kinetic design of excitatory synapses. Nature Reviews Neuroscience 6, 841-849 (2005).

17. Willard, S. S. \& Koochekpour, S. Glutamate, glutamate receptors, and downstream signaling pathways. International Journal of Biological Sciences 9, 948-959 (2013).

18. Kandel, E. R., Schwartz, J. H., Jessell, T. M., Siegelbaum, S. A. \& Hudspeth, A. J. Principles of Neural Science 5th Edition. (McGraw Hill, 2013).

19. Bröer, S. \& Brookes, N. Transfer of glutamine between astrocytes and neurons: Glutamine transfer between astrocytes and neurons. Journal of Neurochemistry 77, 705-719 (2001).

20. Edwards, R. H. The Neurotransmitter Cycle and Quantal Size. Neuron 55, 835-858 (2007). 
21. Shen, K. \& Scheiffele, P. Genetics and Cell Biology of Building Specific Synaptic Connectivity. Annual Review of Neuroscience 33, 473-507 (2010).

22. Tzingounis, A. V. \& Wadiche, J. I. Glutamate transporters: Confining runaway excitation by shaping synaptic transmission. Nature Reviews Neuroscience 8, 935-947 (2007).

23. Choi, D. W. \& Rothman, S. M. The Role of Glutamate Neurotoxicity in HypoxicIschemic Neuronal Death. Annu. Rev. Neurosci. 171-182 (1990).

24. Meldrum, B. S. Glutamate as a neurotransmitter in the brain: review of physiology and pathology. The Journal of nutrition 130, 1007S-1015S (2000).

25. Ventura, R. \& Harris, K. M. Three-dimensional relationships between hippocampal synapses and astrocytes. The Journal of neuroscience : the official journal of the Society for Neuroscience 19, 6897-6906 (1999).

26. Reichenbach, A., Derouiche, A. \& Kirchhoff, F. Morphology and dynamics of perisynaptic glia. Brain Research Reviews 63, 11-25 (2010).

27. Bliss, T. V. P. \& Collingridge, G. L. A synaptic model of memory: long-term potentiation in the hippocampus. Nature 361, 31-39 (1993).

28. Barbour, B. \& Häusser, M. Intersynaptic diffusion of neurotransmitter. Trends in Neurosciences vol. 20 377-384 (1997).

29. Clements, J. D. Transmitter timecourse in the synaptic cleft: its role in central synaptic function. Trends in Neurosciences 19, 163-171 (1996).

30. Franks, K. M., Bartol, T. M. \& Sejnowski, T. J. A Monte Carlo Model Reveals Independent Signaling at Central Glutamatergic Synapses. Biophysical Journal 83, 2333-2348 (2002). 
31. Syková, E. \& Nicholson, C. Diffusion in Brain Extracellular Space. Physiological Reviews 88, 1277-1340 (2008).

32. Nicholson, C. \& Hrabětová, S. Brain Extracellular Space: The Final Frontier of Neuroscience. Biophysical Journal 113, 2133-2142 (2017).

33. Korogod, N., Petersen, C. C. H. \& Knott, G. W. Ultrastructural analysis of adult mouse neocortex comparing aldehyde perfusion with cryo fixation. eLife (2015) doi:10.7554/eLife.05793.

34. Tønnesen, J., Inavalli, V. V. G. K. \& Nägerl, U. V. Super-Resolution Imaging of the Extracellular Space in Living Brain Tissue. Cell 172, 1108-1121 (2018).

35. Hrabětová, S., Cognet, L., Rusakov, D. A. \& Nagerl, U. V. Unveiling the Extracellular Space of the Brain: From Super-resolved Microstructure to In Vivo Function. Journal of Neuroscience 38, 9355-9363 (2018).

36. Rasmussen, M. K., Mestre, H. \& Nedergaard, M. The glymphatic pathway in neurological disorders. The Lancet Neurology 17, 1016-1024 (2018).

37. Carare, R. O. et al. Solutes, but not cells, drain from the brain parenchyma along basement membranes of capillaries and arteries: significance for cerebral amyloid angiopathy and neuroimmunology. Neuropathology and Applied Neurobiology 34, 131-144 (2008).

38. Bakker, E. N. T. P. et al. Lymphatic Clearance of the Brain: Perivascular, Paravascular and Significance for Neurodegenerative Diseases. Cellular and Molecular Neurobiology 36, 181-194 (2016).

39. Arbel-Ornath, M. et al. Interstitial fluid drainage is impaired in ischemic stroke and Alzheimer's disease mouse models. Acta Neuropathologica 126, 353-364 (2013). 
40. Tarasoff-Conway, J. M. et al. Clearance systems in the brain-implications for Alzheimer disease. Nature Reviews Neurology 11, 457-470 (2015).

41. Boland, B. et al. Promoting the clearance of neurotoxic proteins in neurodegenerative disorders of ageing. Nature Reviews Drug Discovery 17, 660 (2018).

42. Da Mesquita, S., Fu, Z. \& Kipnis, J. The Meningeal Lymphatic System: A New Player in Neurophysiology. Neuron 100, 375-388 (2018).

43. Simon, M. J. \& Iliff, J. J. Regulation of cerebrospinal fluid (CSF) flow in neurodegenerative, neurovascular and neuroinflammatory disease. Biochimica et Biophysica Acta (BBA) - Molecular Basis of Disease 1862, 442-451 (2016).

44. Iliff, J. J. et al. Cerebral Arterial Pulsation Drives Paravascular CSF-Interstitial Fluid Exchange in the Murine Brain. Journal of Neuroscience 33, 18190-18199 (2013).

45. Mestre, H., Kostrikov, S., Mehta, R. I. \& Nedergaard, M. Perivascular spaces, glymphatic dysfunction, and small vessel disease. Clinical Science 131, 2257-2274 (2017).

46. Abbott, N. J. Evidence for bulk flow of brain interstitial fluid: significance for physiology and pathology. Neurochemistry International 45, 545-552 (2004).

47. Jiang, J. \& Amara, S. G. New views of glutamate transporter structure and function: Advances and challenges. Neuropharmacology 60, 172-181 (2011).

48. Kanner, B. I. Structure and Function of Sodium-coupled GABA and Glutamate Transporters. The Journal of Membrane Biology 213, 89-100 (2006).

49. Kanai, Y. et al. The SLC1 high-affinity glutamate and neutral amino acid transporter family. Molecular Aspects of Medicine 34, 108-120 (2013).

50. Vandenberg, R. J. \& Ryan, R. M. Mechanisms of Glutamate Transport. Physiological Reviews 93, 1621-1657 (2013). 
51. Sheldon, A. L. \& Robinson, M. B. The role of glutamate transporters in neurodegenerative diseases and potential opportunities for intervention. Neurochemistry International 51, 333-355 (2007).

52. Chen, I. et al. Glutamate transporters have a chloride channel with two hydrophobic gates. Nature 591, 327-331 (2021).

53. Yernool, D., Boudker, O., Jin, Y.\& Gouaux, E. Structure of a glutamate transporter homologue from Pyrococcus horikoshii. Nature 431, 811-8 (2004).

54. Krishnamurthy, H., Piscitelli, C. L. \& Gouaux, E. Unlocking the molecular secrets of sodium-coupled transporters. Nature 459, 347 (2009).

55. Boudker, O., Ryan, R. M., Yernool, D., Shimamoto, K. \& Gouaux, E. Coupling substrate and ion binding to extracellular gate of a sodium-dependent aspartate transporter. Nature $\mathbf{4 4 5}$, $387-393$ (2007).

56. Bastug, T. et al. Position of the Third $\mathrm{Na}+$ Site in the Aspartate Transporter Glt Ph and the Human Glutamate Transporter ,. PLoS ONE 7, 13-17 (2012).

57. Reyes, N., Ginter, C. \& Boudker, O. Transport mechanism of a bacterial homologue of glutamate transporters. Nature 462, 880-885 (2009).

58. Altun, Z. F. et al. Wormatlas. http://www.wormatlas.org/ (2002).

59. Brockie, P. J., Madsen, D. M., Zheng, Y., Mellem, J. \& Maricq, A. V. Differential expression of glutamate receptor subunits in the nervous system of Caenorhabditis elegans and their regulation by the homeodomain protein UNC-42. The Journal of neuroscience : the official journal of the Society for Neuroscience 21, 1510-22 (2001).

60. Brockie, P. Ionotropic glutamate receptors: genetics, behavior and electrophysiology. WormBook (2006) doi:10.1895/wormbook.1.61.1. 
61. Serrano-Saiz, E. et al. Modular control of glutamatergic neuronal identity in C.elegans by distinct homeodomain proteins. Cell 155, 1-27 (2013).

62. Mano, I., Straud, S. \& Driscoll, M. Caenorhabditis elegans glutamate transporters influence synaptic function and behavior at sites distant from the synapse. Journal of Biological Chemistry 282, 34412-34419 (2007).

63. Katz, M., Corson, F., Iwanir, S., Biron, D. \& Shaham, S. Glia Modulate a Neuronal Circuit for Locomotion Suppression during Sleep in C. elegans. Cell Reports 22, 2601-2614 (2018).

64. Mano, I. \& Driscoll, M. Caenorhabditis elegans glutamate transporter deletion induces AMPA-receptor/adenylyl cyclase 9-dependent excitotoxicity. Journal of Neurochemistry 108, 1373-1384 (2009).

65. White, J. G., Southgate, E., Thomson, J. N. \& Brenner, S. The Structure of the Nervous System of the Nematode Caenorhabditis elegans. Philosophical Transactions of the Royal Society of London 314, 1-340 (1986).

66. Cook, S. J. et al. Whole-animal connectomes of both Caenorhabditis elegans sexes. Nature 571, 63-71 (2019).

67. Brittin, C. A., Cook, S. J., Hall, D. H., Emmons, S. W. \& Cohen, N. A multi-scale brain map derived from whole-brain volumetric reconstructions. Nature 591, 105-110 (2021).

66. Shipley, F. B., Clark, C. M., Alkema, M. J. \& Leifer, A. M. Simultaneous optogenetic manipulation and calcium imaging in freely moving C. elegans. Front. Neural Circuits $\mathbf{8}$, (2014). 
67. Larsch, J., Ventimiglia, D., Bargmann, C. I. \& Albrecht, D. R. High-throughput imaging of neuronal activity in Caenorhabditis elegans. Proc. Natl. Acad. Sci. 110, E4266-E4273 (2013).

68. Piggott, B. J., Liu, J., Feng, Z., Wescott, S. A. \& Xu, X. Z. S. The Neural Circuits and Synaptic Mechanisms Underlying Motor Initiation in C. elegans. Cell 147, $922-933$ (2011).

69. Marvin, J. S. et al. An optimized fluorescent probe for visualizing glutamate neurotransmission. Nat. Methods 10, 162-170 (2013). 


\section{Chapter 2}

Novel Strategies for Glutamate Clearance in the Glia-Deprived Synaptic Hub of C. elegans

A manuscript (in revision)

Joyce Chan ${ }^{1,2}$, Kirsten KyungHwa Lee ${ }^{1}$, Irving Estevez ${ }^{1,3}$, Jenny Chan Ying Wong ${ }^{1,3}$, Paola Morocho ${ }^{1,4}$, and Itzhak Mano ${ }^{1,2 *}$.

1) Cellular, Molecular, and Biomedical Science, CUNY School of Medicine, City College of New York (CCNY), The City University of New York (CUNY).

2) $\mathrm{PhD}$ Program in Biology, The CUNY Graduate Center

3) M.A. Program in Biology, CCNY.

4) Undergraduate Program in Biology, CCNY.

* Corresponding author:

Dr. Itzhak Mano

Cellular, Molecular, and Biomedical Science, CUNY School of Medicine, City College of New York (CCNY), The City University of New York (CUNY).

Center for Discovery \& Innovation (CDI), Room 3-382

85 St. Nicholas Terrace, New York, NY 10031

E-mail: imano@med.cuny.edu Phone: 212-650-7965

\section{Authors contribution}

Itzhak Mano, Joyce Chan, Kirsten KyungHwa Lee, and Irving Estevez designed the project, analyzed the data, and wrote the manuscript; Joyce Chan, Kirsten KyungHwa Lee, and Irving Estevez produced transgenic constructs and strains; Kirsten KyungHwa Lee performed the initial imaging experiments, while Joyce Chan obtained nearly all imaging data presented here; Jenny Chan Ying Wong and Paola Morocho performed the behavioral experiments. 


\subsection{Abstract}

In the mammalian CNS, different GluTs are expressed in different regions and cell types (e.g., glia vs. neurons), differing in proximity from Glu synapses ${ }^{1}$. Though these GluTs also differ slightly in their functional properties, it remains unclear what is the significance or "necessity" of using different GluTs. Though the importance of the ECS, interstitial fluid, and the glymphatic system has recently been recognized, it remains unclear how bulk flow contributes to preventing spillover and ensuring effective clearance ${ }^{2}$. These questions can be studied with great precision in C. elegans ${ }^{3}$, facilitated by its fully documented circuit wiring diagram (a map of synapses at the EM level) and accessibility to a wealth of well-established molecular tools. By stimulating specific circuits, we quantify both extracellular Glu levels using iGluSnFR ${ }^{4,5}$, and postsynaptic responses using $\mathrm{GCaMP}^{6}$ in intact animals. Additionally, "promoter swapping" (swapping the expression of these GluTs, i.e., ectopically expressing one transporter instead of the other) of proximal and distal GluTs provides insight as to whether functional properties exist between these different GluT or if their differences are actually attributed to expression pattern. 


\subsection{Introduction}

2.2.1. The nociceptive ASH circuit and a link to perfusion-mediated clearance. In the $C$. elegans system, the ASH sensory neurons are a bilateral pair of glutamatergic polymodal nociceptive sensory neurons that mediate to avoidance responses from noxious stimuli, including hyperosmolarity, nose touch, high salt and alkaloids (such as quinine) $)^{7,8}$. Upon stimulation, ASH transmits excitatory Glu signals to GluR-expressing postsynaptic target neurons AVA, AVD, and AVE (referred to here as the ASH circuit) and subsequently to motor neurons in order to trigger backwards locomotion (the avoidance response) ${ }^{9}$. Backwards locomotion can also initiate spontaneously in freely-moving worms, in the absence of noxious stimuli or sensory cues ${ }^{10}$. While interneurons AVA/D/E are important for reversal initiation, they are not essential and worms can still initiate reversal (albeit at reduced frequency) when these neurons are ablated ${ }^{9}$

Asides from the glutamatergic nature of ASH signaling and the well-characterized behaviors mediated by the circuit, another relevant key feature of the ASH circuit is the location of the synapses at the inner rim of the nerve ring. At the anterior of the nematode exists the accessory pseudocoelom, a narrow space containing interstitial fluid is formed between the pharynx and basal laminae ${ }^{7}$. This pseudocoelomic fluid is directly connected to the main reservoir of body fluids (the pseudocoelom) ${ }^{7}$. Since the basal membrane anatomically separating tissues in this area is permeable to small molecules, solutes in the pseudocoelomic fluid in this region might have unfettered access to the inner rim of the nerve ring. We considered a potential link between the ability of distal GluTs to control of ambient Glu concentration in pseudocoelomic body fluids and the location of ASH->AVA/AVD/AVE synapses closer to the inner rim. It remains unclear how the distal GluTs expressed on the canal cell might 
preferentially affect ASH connections, located closer to the pharynx, yet we observe later on in our behavioral studies that loss of function of distal GluTs significantly impairs ASH-mediated responses of the nematode. Since the capillaries of canal cells expressing the glt-3, glt-6, and glt7 are directly exposed to pseudocoelomic body fluids, distal GluTs are likely to regulate ambient Glu concentrations. It is possible that the pulsations of the pharynx and the increased mobility in the nematode head region facilitates perfusion of the inner rim with body fluids. Such perfusion can replace Glu-rich synaptic fluids with fresh body fluids containing ambient Glu levels. If normal Glu clearance in the inner rim of the nerve ring benefits from mechanical washout and perfusion by body fluids, then absence of the distal GluTs will increase ambient Glu concentration, hampering Glu diffusion from these synapses under conditions of increased Glu synaptic activity. This led us to hypothesize that agitation of body fluids and perfusion of the inner rim of the nerve ring underlie the privileged effect of distal GluTs on the ASH synapses.

\subsubsection{Sequence differences between proximal vs distal GluTs may have an effect on ligand}

affinity and transport capacity. Sequence difference and the resulting possible structural differences between proximal and distal GluTs may correlate to potential differences in affinity and capacity. TM7 and HP2 display residue differences between the two types. Both domains are directly involved substrate transport, and are predicted to confer unique affinities for $\mathrm{Glu}^{11}$. The unusual sequence in the binding sites (particularly the site for $\mathrm{Na}^{+}$) seen in distal GluTs may reduce the driving force of the transporter, increase $\mathrm{K}_{\mathrm{m}}$, and reduce overall affinity (Figure 2) ${ }^{12}$. Reduced transporter affinity is usually coupled with increased turn-over rate (transport capacity). A blend of low-affinity/high-capacity transporters and high-affinity/low-capacity transporters 
facilitate quick uptake when the solute is abundant, and slow uptake of residual solute when substrate is scarce. Examples can be seen with bacterial adjustment to environments in flux ${ }^{13}$, renal glucose reabsorption ${ }^{14}$, and hepatic uptake of cationic amino acids ${ }^{15}$. Additionally, differences have been reported on the ability of some GluTs to mediate uncoupled $\mathrm{Cl}^{-}$ conductance, effectively turning these mediocre transporters into effective Glu-gated $\mathrm{Cl}^{-}$ channels ${ }^{16-18}$. This property is especially important in GluTs expressed on presynaptic terminals, where they may functions as "sensors" in a feedback mechanism to curtail excessive Glu release (i.e., as in the retina, where large amounts of Glu are tonically released in a graded fashion) ${ }^{12}$. However, it remains unclear how the ensemble of different GluTs act in concert to regulate overall Glu physiology in the nervous system ${ }^{12}$. We therefore want to explore the functional properties of the nematode GluTs, and determine if expression patterns correlate with their different functional properties, creating a range of GluT functions that address the different needs of Glu clearance in the worm. Such differences may have the combined effect of providing a wide range of clearance capabilities for different glutamatergic events. We speculate that GluTs located away from the synapse deal with large, Glu surges that may come from global nerve ring signaling, while high affinity/low capacity GluTs manage and maintain low Glu concentrations at the vicinity of specific synapses. GluTs with high $\mathrm{Cl}^{-}$conductance can curtail further Glu signaling. An understanding of how the activities of different GluTs modulate Glu physiology may be useful in future studies of GluTs in higher organisms. While bacterial and mammalian GluTs have been relatively well-characterized, no attempts have yet been made to model nematode GluTs. We performed sequence alignments to compare the conservation of residue identity in the nematode GluTs against other known related amino acid transporters. With help from D. Jeruzalmi (a structural biologist in CCNY), I 
submitted GluT sequences to the I-TASSER program to build predictive 3D models and identify putative ligand binding sites and interacting side chains ${ }^{19-21}$. I was able to build nematode GluTs around hEAAT1, then visualize structures in PyMOL modeling software ${ }^{22}$ (Figure 5).

While I had observed especially high conservation in the overall structure between the bacterial and nematode transporters from the 3D models, there were visible differences in a key Glu- and $\mathrm{Na}^{+}$- binding motif in TM7: distal GluTs (GLT-3, GLT-6, and GLT-7) display deviations in sequence at the 'NMDGT' motif, deviations that are not observed in the proximal GluTs (GLT-1 and GLT-4) (Figure 2). We propose that such differences in sequence affect transporter affinities for substrates. While the high conservation of the 'NMDGT' motif across species may suggest that transporters with any deviation from the motif sequence can have a negative impact on clearance, it is entirely possible that the deviations observed at this motif in distal GluTs can enhance transporter activity.

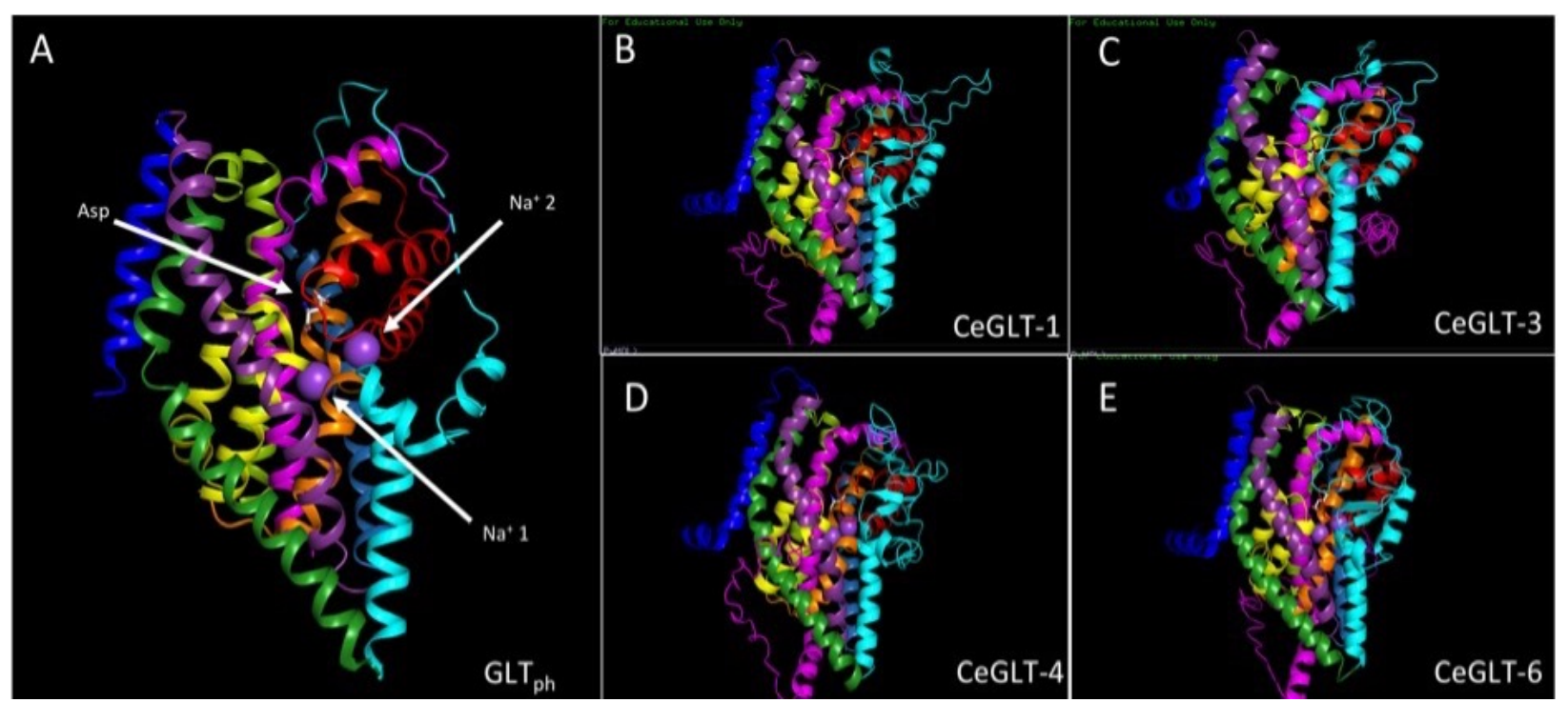

Figure 5. Predictive modeling of $C$. elegans GluTs reveals high structural similarities with bacterial GitPh. White arrows indicate the position of substrates bound to GltPh. Core binding domains are colored as follows: HP1 (yellow), TM7 (orange), HP2 (red), TM8 (magenta). For each panel, the following structures are depicted: GltPh (A), C. elegans GLT-1 (B), C. elegans GLT-3 (C), C. elegans GLT-4 (D), and C. elegans GLT-6 (E). 


\subsection{Results}

\subsubsection{Behaviors mediated by the avoidance circuit are strongly sensitive to manipulation of distal GluTs.}

Past experiments in our lab have centered on aversive responses mediated by the glutamatergic nociceptive neuron ASH and its GluR-expressing targets, interneurons AVA, AVD, and AVE (which comprise the ASH circuit) ${ }^{23,24}$. My results build off of the work of former lab member Jenny Wong, who extended these behavioral analyses to all GluT mutants, and former postdoc KyungWha Lee, who set up a microfluidics-based imaging system ${ }^{6}$ and used AVA-expressed GCaMP ${ }^{25-27}$ to provide preliminary documentation on the roles of different GluTs in clearance of AVA synapses. We find that KO of the distal GluT glt-3 shortens the duration of spontaneous forward mobility, while elimination of either glt-3 alone or the glt-3, glt-6, \& glt-7 KO combination disrupts nose touch response (Figure 6). Both behaviors are known to be mediated by the ASH circuit 23,24 . 


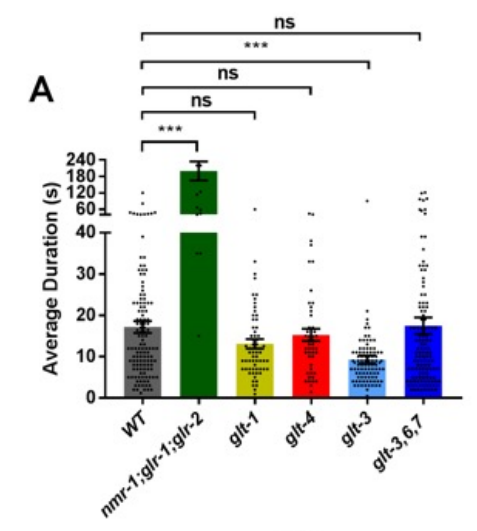

Figure 6. Distal GluT KOs disrupt behaviors mediated by the ASH $>$ AVA avoidance circuit.

(A) Duration of forward runs in spontaneous mobility assay. glt-3 (distal GluT KO) animals are impaired in forward run duration, although the duration of forward run of proximal GluT KO animals ( $g l t-1$,or $g l t-4)$ is intact. The $n m r-1 ; g l r-2 ; g l r-1$ strain (where all ionotropic GluR responses of the avoidance circuit are eliminated) is used as a control where Glu signaling is reduced, resulting in excessive forward mobility.

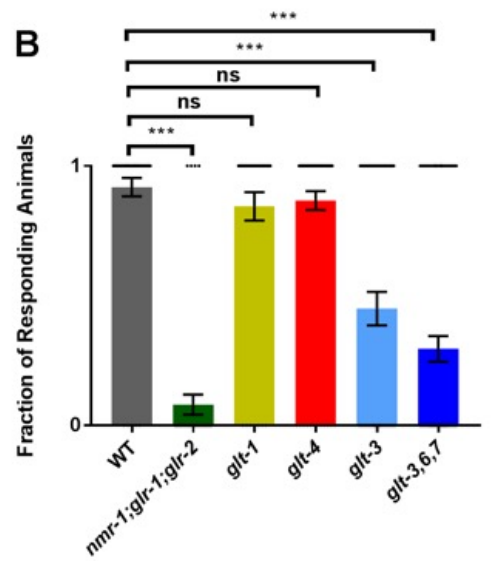
(B) Nose touch response of distal GluT KOs, glt-3 and glt-3, glt-6, glt-7 $\mathrm{KO}$ is reduced. Colored bars represent the fraction of animals responding to nose touch. Both underactive (nmr-1; glr-2; glr-1 animals) and overactive (glt-3 and glt-3, glt-6, glt-7 animals) Glu signaling causes impaired response to nose touch. Significance of differences from control (WT, N2 strain) mean (A) or distribution (B) is indicated by asterisks. ${ }^{* *} P=0.10 ; * * * P<0.001$. Student's t-test with Welch's correction was used in both (A) and (B). Error bars indicate SEM; $n=$ 15-120 per genotype (A), $\mathrm{n}=45-90$ per genotype $(\mathbf{B})$.

\subsubsection{Hyperstimulation of the avoidance circuit in distal GluT KO animals is evident in} calcium and glutamate reporter recordings.

Though behavioral studies in C. elegans often reflect actual changes in functional circuitry, we wanted to study these changes at the neurophysiological level. To examine the effect of GluT $\mathrm{KO}$ on physiological responses of the postsynaptic neurons in the avoidance circuit more directly, we set up a nematode microfluidics and imaging system similar to the one pioneered by the Bargmann $1 a b^{6,25,28,29}$. In this setup, the worm is restrained in a microfluidic chip in absence of paralytic agents, restricting exposure of stimulant solutions to the nose (and consequently, sensory amphid neuron endings). The nematode's transparency allows for non-invasive, in vivo recording of physiological neuronal activity, which we monitor using genetically encoded fluorescent $\mathrm{Ca}^{2+}$ sensor (GCaMP) expressed in identified neurons (as a transgene driven by a cell-specific promoter). This system provides us an easily accessible window into neuronal 
activity in specific postsynaptic neurons in intact animals without compromising the hydrodynamics of interstitial fluids or changes in concentrations of our neurotransmitter of interest. We used GCaMP expressed in AVA (the main mediator of avoidance responses) to monitor the activity of this neuron in response to ASH stimulation by high concentration glycerol (Figure 7A, B), measuring changes in whole-soma GCaMP fluorescence, which is correlated with membrane depolarization in this cell ${ }^{9}$. We used the QW625 (zfIs42[P rig3::GCaMP3::SL2::mCherry]) strain ${ }^{30}$ due to its inter-animal consistency in GCaMP intensity (as a strain where the transgenic reporter is integrated into the genome). Though ASH-specific nociceptive stimuli are known to produce measurable GCaMP responses in AVA in freely moving animals ${ }^{9,28,31}$, the consistency of this response is reduced in animals restrained in the microfluidic chip (in line with observations made by other labs on AVA responses in restrained vs free animals, S. Chalasani, personal communication). A small AVA response is observed when the proximal GluTs $g l t-1$ or $g l t-4$ are eliminated. In sharp contrast, elimination of the distal GluTs $g l t-3$, glt-6, \& glt-7 elicited a very prominent postsynaptic AVA response to ASH stimulation with glycerol (Figure 7A, B). KO of distal GluT therefore results in an exaggerated AVA response to nociceptive stimulation that is believed to trigger Glu release from their presynaptic partner, ASH. 

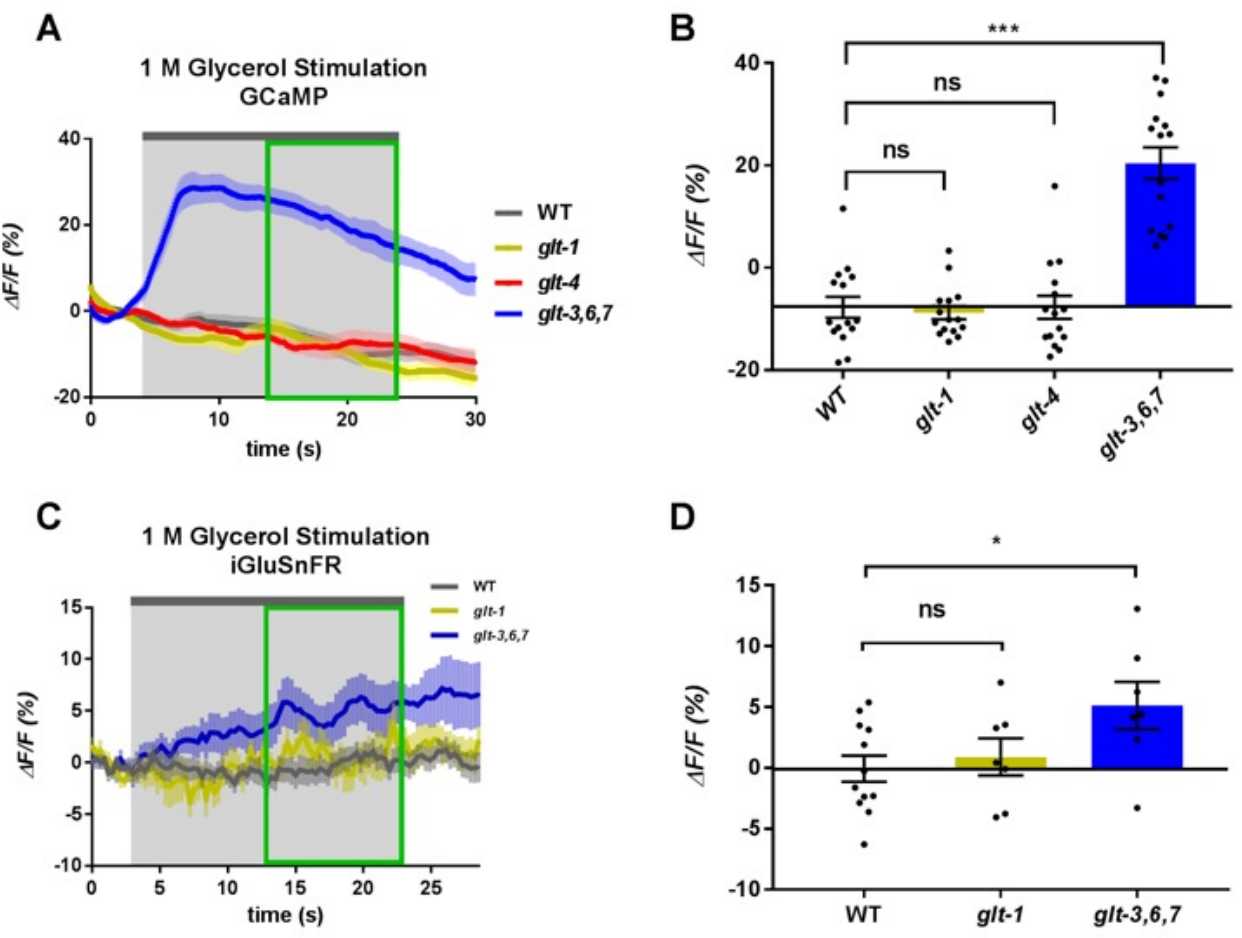

Figure 7. Distal GluT KO causes exaggerated response of AVA to ASH stimulation, and is accompanied by increase of Glu signals on AVA neurites.

A,B) When distal GluT are absent, stimulation of ASH causes an exaggerated response in AVA.

WT and GluT KO animals expressing GCaMP3 in AVA neurons were imaged in the cell soma. Light gray shading indicates $20 \mathrm{~s}$ period of exposure to a $1 \mathrm{M}$ glycerol stimulus, beginning at $t=4 \mathrm{~s}$. The first $4 \mathrm{~s}$ of the recording was averaged to serve as the baseline fluorescence, $F_{0}$. Green box indicates a window of $10 \mathrm{~s}$ after neural response to stimulation reached a relative steady state, for which the fluorescence change $(\Delta F)$ was averaged and presented in the bar graphs. ${ }^{* *} P=0.10, * * * P<0.001$, ANOVA with Bonferroni correction. Light colored shading around each curve and the error bars on bar graphs indicate SEM. $\mathrm{n}=15$ for each strain. C,D) Stimulation of ASH in distal GluT KOs promote accumulation of Glu at the AVA synapse. WT and GluT KO animals expressing iGluSnFR in AVA neurons were imaged in the area of the proximal section on the AVA neurites known to receive ASH inputs. Light gray shading indicates 20 s period of exposure to a $1 \mathrm{M}$ glycerol stimulus, beginning at $t=3 \mathrm{~s}$. The first $3 \mathrm{~s}$ of the recording was averaged to serve as the baseline fluorescence, $F_{0}$. Green box indicates a window of $10 \mathrm{~s}$ after neural response to stimulation reached a relative steady state, for which the fluorescence change $(\Delta \mathrm{F})$ was averaged and presented in the bar graphs. *** $P$ $<0.001$, ANOVA with Bonferroni correction. Light colored shading around each curve and the error bars on bar graphs indicate SEM. $\mathrm{n}=5-6$ for each strain.

We next sought to determine if the increased postsynaptic responses in AVA in distal GluT KO animals arose directly from increased concentration of perisynaptic Glu around AVA's neurites, and not from heightened response sensitivity of AVA, or from indirect connections (such as gap junctions) from other neurons affecting AVA. To directly track effects on perisynaptic Glu concentrations we used the Glu-sensitive extracellular sensor iGluSnFR, 
expressed specifically in AVA neurons in CX14652 (kyEx4787 [P rig-3 $_{3}: i G l u S n F R, ~ u n c-$ $122:: d s R e d]$ ) animals ${ }^{5}$, and measured changes in florescence on AVA's neurite segment that is known to receive the ASH input ${ }^{5,32,33}$. Measuring changes in iGluSnFR fluorescence in AVA neurites in the nerve ring is technically challenging, especially when the animal continues to perform pharyngeal pumping and local pharynx movements in the absence of pharmacological paralyzing agents. Nonetheless, in response to nociceptive stimulation of ASH we saw a small increase in Glu readouts in AVA's nerve ring neurites in glt-1 mutant and a dramatic increase in glt-3 mutants (Figure 7 C, D).

We next wanted to establish that the exaggerated response to nociceptive stimulation seen in postsynaptic AVA neurons in distal GluT KO animals is due to release of Glu from ASH, the canonical presynaptic sensory neuron in this circuit, and not from other sensory neurons that might now ectopically feed into the ASH-AVA circuit. We recorded AVA GCaMP responses in animals containing a loss-of-function mutation of the vesicular GluT (vGluT) EAT-4 (the most prominent of three vGluTs/SLC17A7 orthologs found in the nematode), which severely impacts synaptic Glu neurotransmission in the C. elegans system ${ }^{34}$. This systemic elimination of EAT-4 resulted in loss of AVA responses in wild type (WT), glt-1, and $g l t-4$ animals, and erased the exaggerated AVA response typically seen in $g l t-3, g l t-6, \&$ glt-7 mutants (Figure 8 A,B). However, a late, residual response still remains in $g l t-3,6, \& 7$ mutants, suggesting that remnant Glu release still exists in spite of the animal-wide -absence of EAT-4, a notion that was also indicated in our previous studies ${ }^{35}$. Such remnant Glu release can potentially be attributed to the activity of the remaining two vGluTs ${ }^{36,37}$, or to the activity of other members of the SLC17 transporter family ${ }^{38,39}$, such as slc-17.2. 

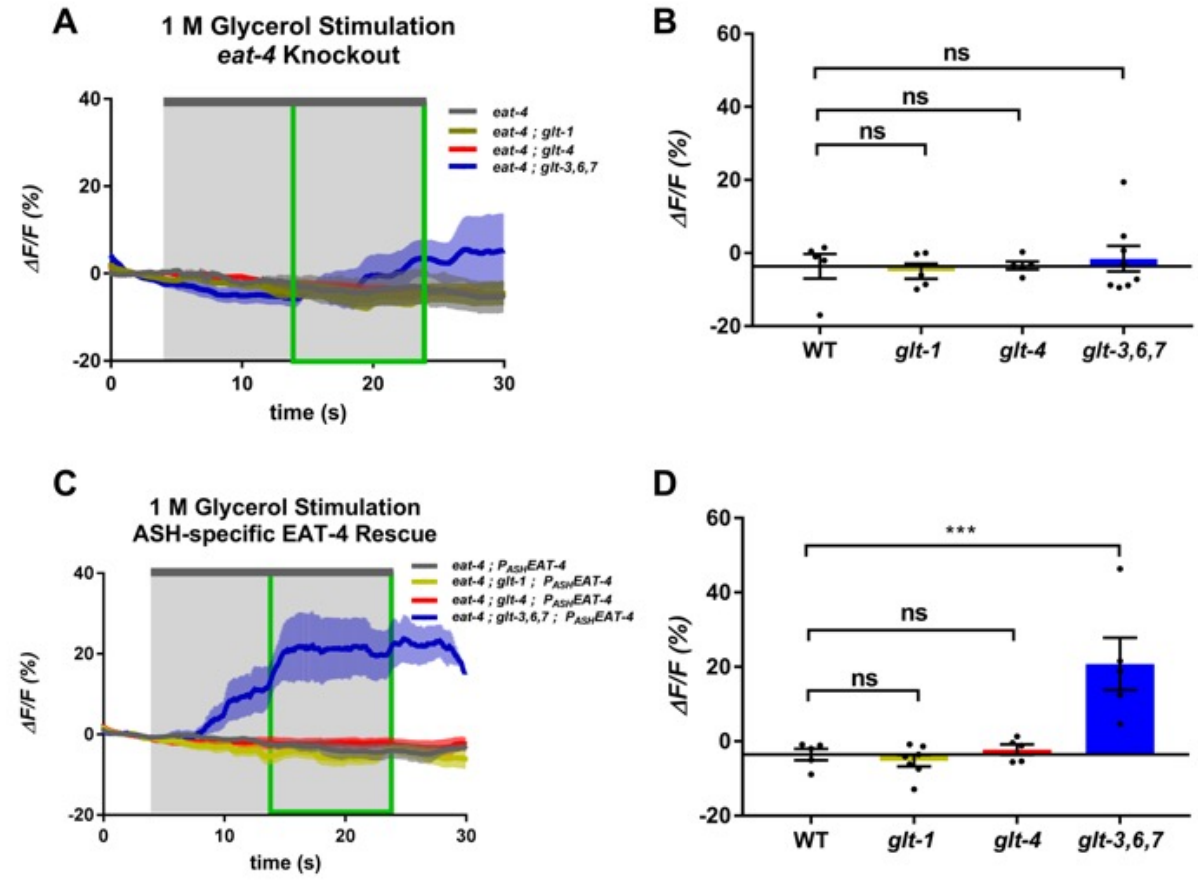

Figure 8. ASH is the source of Glu signals that directly result in the exaggerated AVA responses seen in Distal GluT KO animals in response to stimulation by $1 \mathrm{M}$ Glycerol.

$\mathrm{A} ; \mathrm{B})$ The exaggerated responses of AVA to stimulation of ASH depend (at least primarily) on the vGluT EAT-4. Glycerol-induced changes in GCaMP3 fluorescence of AVA neurons in WT and GluT KO animals with LOF mutations of vGluT eat-4. Average traces of changes in GCaMP3 fluorescence and averaged steady-state responses to $1 \mathrm{M}$ Glycerol stimulation are analyzed similarly to those in Fig 5A, B. ${ }^{* * *} P<0.001$, ANOVA with Bonferroni correction. Light colored shading around curves and the error bars on bar graphs indicate SEM. $\mathrm{n}=5-8$ for each strain.

$\mathrm{C}$;D) ASH-specific rescue of Glu release in Distal GluT KO animals fully restores response in AVA in a mutant eat-4 background. Glycerol-induced changes in GCaMP3 fluorescence of AVA neurons in WT and GluT KO animals with LOF mutations of vGluT eat-4. Extrachromosomal constructs allow for ASH-specific expression of wild-type eat-4. Average traces of changes in GCaMP3 fluorescence and averaged steady-state responses to $1 \mathrm{M}$ Glycerol stimulation are analyzed similarly to those in Fig 5A, B. ${ }^{* * *} P<0.001$, ANOVA with Bonferroni correction. Light colored shading around curves and the error bars on bar graphs indicate SEM. $\mathrm{n}=5$ for each strain.

We then used an extrachromosomal transgene expressing eat-4 cDNA under ASH-specific promoter $\left(P_{\text {sra- } 6}\right)^{9}$ to restore EAT-4 expression specifically in the ASH neurons. We observed that transgenic expression of EAT-4 in ASH is sufficient for restoring the exacerbated response in glt-3,6, \& 7 mutant animals, to a level similar to what we had seen previously in $g l t-3,6, \& 7$ mutant animals expressing endogenous, WT EAT-4 (Figure 8C, D). Though there is a notable delay in the response of glt-3,6, \& 7 mutant animals with transgenic ASH-specific rescue to 
nociceptive stimulation (compared to animals with endogenous eat-4), this difference can be attributed to variations in the degree of eat-4 cDNA expression in ASH neurons between individual transgenic animals (which is typical of extrachromosomal transgene expression).

Taken together, the GCaMP and iGluSnFR -based imaging data correlate with the behavioral observations, and provides direct evidence (at the level of cell-specific Glu release, actual changes in perisynaptic Glu concentrations, and postsynaptic response physiology) that elimination of distal Glu clearance strongly potentiates Glu signaling between ASH and AVA in the avoidance circuit, while elimination of proximal GluTs produces no effect at the physiological levels. Although distal GluTs have sequence divergence in the core transport domain (compared to other members of the SLC1A family) ${ }^{35}$, they are still bona fide transporters of Glu, and their KO causes Glu accumulation in AVA synapses. These observations strongly support that the nature of the signals resulting in exacerbated AVA response in distal glt-3,6, \& 7 mutants are: A) glutamatergic, and B) originating from ASH. Unlike proximal GluTs, distal GluTs seem to play a major role in clearing Glu from ASH -> AVA synapses. Given the challenges in measuring changes in perisynaptic Glu concentrations with iGluSnFR in nonparalyzed animals, we continued our analyses mostly using GCaMP imaging to detect changes in intracellular $\mathrm{Ca}^{2+}$ concentrations in the soma as a reasonable reporter of synaptic excitation. As we gain insight into the preferential contribution of distal vs proximal GluT to Glu clearance in the avoidance circuit, we became interested in investigating the contribution of different GluTs to the activity of a neighboring circuit that mediates chemoattraction. 


\subsubsection{Reduction of proximal Glu clearance generates avoidance response to low concentration of $\mathrm{NaCl}$.}

Low $\mathrm{NaCl}$ concentrations $(<200 \mathrm{mM})$ are chemoattractive: the bilateral ASE sensory neurons are sensitive to low $\mathrm{NaCl}$ concentrations and transmit Glu signals to AIB, AIA, and AIY interneurons to regulate chemotaxis. Combined with alternating head movements, ASEL detects an increase in salt concentration ( $\mathrm{NaCl}$ upstep), inhibiting AIBs through Glu-gated $\mathrm{Cl}^{-}$channels such as GLC-3 to suppress reversal. ASER detects a concentration decrease ( $\mathrm{NaCl}$ downstep), stimulating the AIBs through AMPA-Rs (such as GLR-1) and metabotropic GluRs, causing animal reversal ${ }^{40-42}$. In contrast to the sensitivity of ASE neurons to low salt concentrations, high $\mathrm{NaCl}$ concentrations are nociceptive and generate avoidance responses, stimulating the ASH neurons and their associated avoidance circuit ${ }^{8}$. Since the synapses of avoidance and chemoattractive circuits are localized with considerable anatomical proximity in the nerve ring, we sought to determine whether GluTs contribute to the maintenance of these circuits' functional separation, and if GluT KO might cause Glu spillover between these circuits. As a first attempt to find evidence that such a spillover occurs, we tested the possibility that low $\mathrm{NaCl}$ concentrations (which normally stimulate the ASE->AIA/AIB/AIY chemoattractive circuit) might trans-activate the ASH->AVA/AVD/AVE circuit and produce avoidance in absence of GluT function. We measured the concentration dependence of avoidance response to $\mathrm{NaCl}$ using the drop assay ${ }^{8,40}$. We found that mutations in $g l t-1$ or $g l t-4$ (the two proximal GluTs), but not glt-3, glt-6, \& glt-7 KO (the distal GluTs) dramatically changed the normal behavioral doseresponse curve of $\mathrm{NaCl}$ : an extremely small increase in $\mathrm{NaCl}$ concentration $(1 \mathrm{mM})$, which is normally sensed by ASEL and is attractive, becomes chemorepulsive in proximal GluT mutants 
(Figure 9). This result offers a preliminary indication that Glu from the ASEL -> AIA/AIB/AIY circuit might have spilled over to the ASH -> AVA/AVD/AVE circuit.

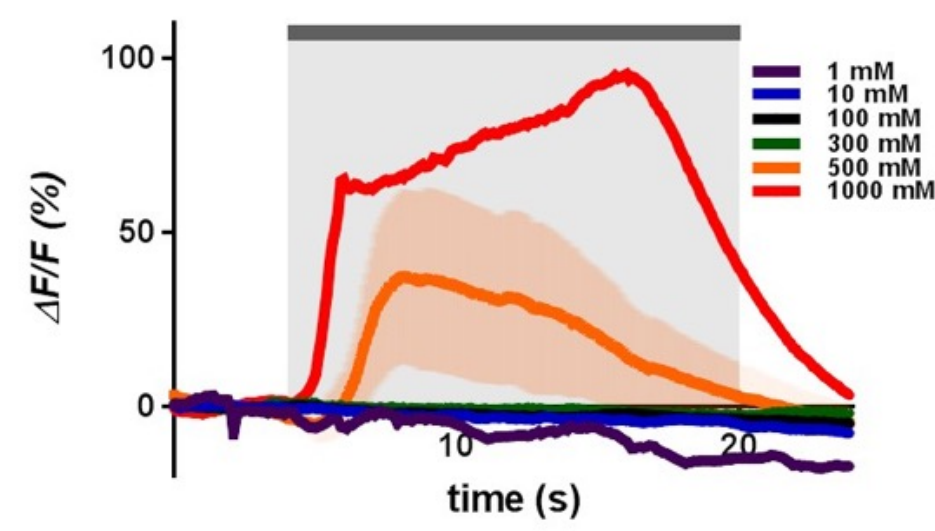

Figure 9. ASH neurons respond only to high concentration $\mathrm{NaCl}$.

Worms with ASH-specific GCaMP3 expression were imaged for changes in ASH activity in response to $\mathrm{NaCl}$ in a range of concentrations. Light gray shading indicates $10 \mathrm{~s}$ of $\mathrm{NaCl}$ application at each concentration, beginning at $t=4 \mathrm{~s}$. The fluorescence change for the first $4 \mathrm{~s}$ before stimulation was averaged to serve as the baseline fluorescence, $\mathrm{F}_{0}$. The light colored shading around each curve indicate SEM. $\mathrm{n}=2-3$ per concentration.

While spillover of Glu between these two circuits is one possible explanation for our observations, other multiple scenarios are possible. One alternative explanation is that indirect signaling between the two circuits through other neuronal connections. However, the contribution of indirect inputs to AVA from neurons such as AIB is considered relatively minor (see WormWeb.org), and should have the opposite effect (as stimulation of ASEL causes inhibition of AIB). Another possibility is that the ASH neurons are in fact also sensitive to low $\mathrm{NaCl}$ concentration and release weak Glu signals onto AVA, signals that can be potentiated by GluT KO. To negate the latter possibility, we directly monitored the activation of ASH by measuring its $\mathrm{Ca}^{2+}$ responses to different concentrations of $\mathrm{NaCl}$, using the $\mathrm{CX} 6632 \mathrm{kyEX728}$ $\left[P_{\text {sra-6: }}: G C a M P\right]$ strain $^{6}$. In accordance with other reports ${ }^{43}$, we find that ASH responds only to very high $\mathrm{NaCl}$ concentrations (Figure 10). These observations further suggest that the avoidance response to $1 \mathrm{mM} \mathrm{NaCl}$ increase in proximal GluT $\mathrm{KO}$ animals is not due to potentiation of previously weak presynaptic release from ASH neurons (that went undetected by postsynaptic measurement in GluT WT animals), and is therefore more likely to represent Glu spillover from other sensory neurons. To affirm this view, we went from testing behavior and 
ASH sensitivity to examining the effect of chemoattractive stimulation on nociceptive responses at the physiological level.

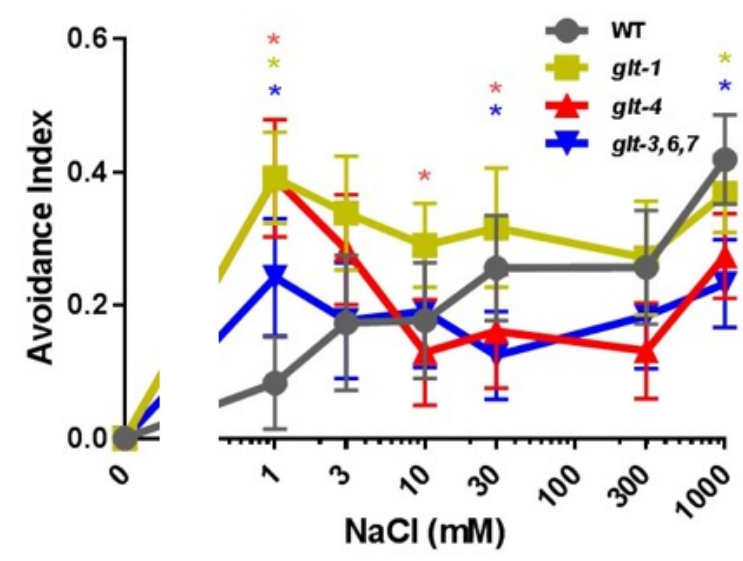

Figure 10. Absence of proximal GluTs abnormally switches worms response to low concentration $\mathrm{NaCl}$ from attraction to avoidance. A drop of buffer containing the indicated concentration of $\mathrm{NaCl}$ was introduced to the tail of a forward-moving worm. Animals presented either an avoidance response (stopping/reversing) or an attraction response (continuing forward traversal). Avoidance index is the percentage of worms that presented an avoidance response. Difference from control (WT) distribution at each $\mathrm{NaCl}$ concentration is indicated by asterisks. $* P<0.05$, Chi-square test. Error bars indicate SEM; $\mathrm{n}=11-62$ for each data point.

\subsubsection{Reduction of proximal Glu clearance causes abnormal AVA activation in response to low $\mathrm{NaCl}$ concentration increase.}

The glutamatergic sensory ASE neuron was previously shown to be responsible for responses to low $\mathrm{NaCl}$ concentrations by triggering activity in the ASE -> AIA/AIB/AIY chemoattractive circuit. This circuit became our leading candidate as the source of Glu in the abnormal avoidance responses we observed in proximal GluT KO animals under stimulation with low $\mathrm{NaCl}$ concentration upstep. We therefore progressed from the behavioral to the physiological level, recording $\mathrm{Ca}^{2+}$ responses to low $\mathrm{NaCl}$ concentration upstep in AVA neurons in GluT KO animals. We find that AVA's neural activity correlates with the avoidance responses in the $\mathrm{NaCl}$ drop assay (Figure 11A, B): while a small upstep in $\mathrm{NaCl}$ dose does not elicit an AVA response in either WT or distal GluT ( $g l t-3$, glt-6, \& glt-7) KO background, reducing proximal Glu clearance by either $g l t-1 \mathrm{KO}$ or $g l t-4 \mathrm{KO}$ results in a robust aberrant $\mathrm{AVA} \mathrm{Ca}^{2+}$ response. A similar effect is also seen with a larger upstep of $50 \mathrm{mM}$ (Figure 12A, B). We could not detect a prominent AVA response to ASER stimulation (by a small $\mathrm{NaCl}$ downstep) in either WT or GluT KO animals (Figure 13). 

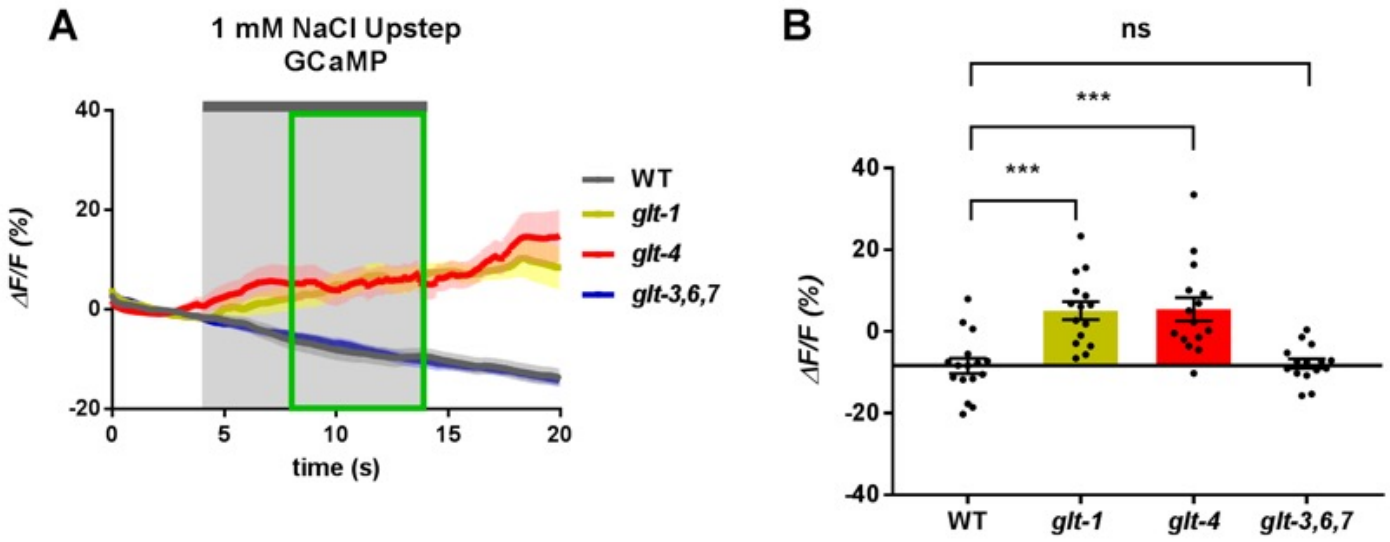

\section{C}

$1 \mathrm{mM} \mathrm{NaCl}$ Upstep iGluSnFR

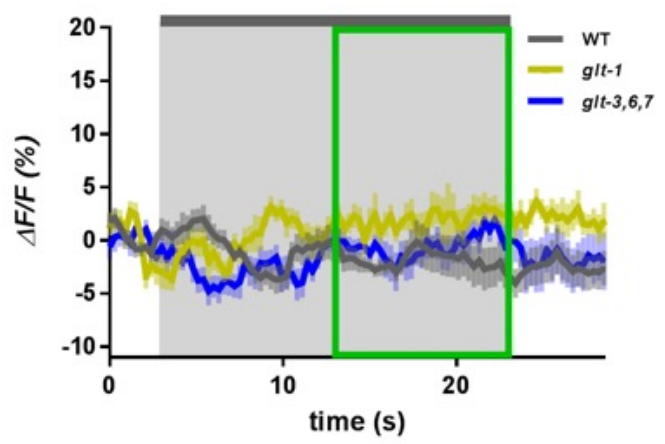

D

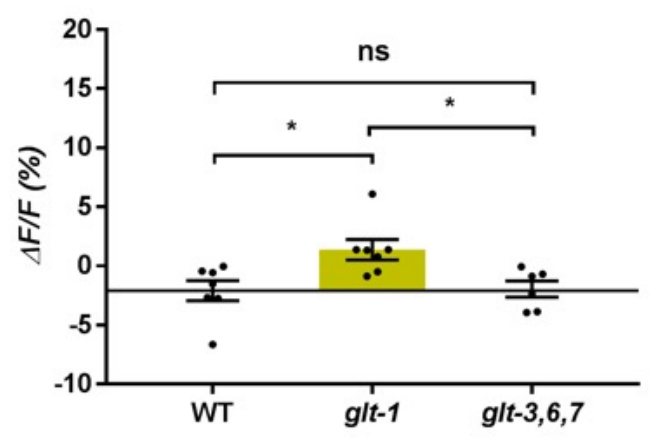

Figure 11. Proximal GluT KOs cause AVA to respond to ASEL stimulation, accompanied by Glu signal on the AVA neurites.

A, B) When proximal GluT are absent, stimulation of ASEL causes an ectopic response in AVA. WT and GluT KOs expressing GCaMP3 in AVA neurons were imaged. Light gray shading indicates $10 \mathrm{~s}$ of 1 $\mathrm{mM} \mathrm{NaCl}$ stimulus, beginning at $t=4 \mathrm{~s}$. The first $4 \mathrm{~s}$ of recording was averaged to serve as the baseline fluorescence, $F_{0}$. Green box indicates the $6 \mathrm{~s}$ when neural activity reached relative steady state, for which the fluorescence change was averaged and presented in the bar graphs. $* * * P<0.001$, ANOVA with Bonferroni correction. Light colored shading around curves and the error bars on bar graphs indicate SEM. $\mathrm{n}=15-16$ for each strain.

C, D) Stimulation of ASEL in GluT KO causes ectopic Glu signal in AVA neurites. WT and GluT KO animals expressing iGluSnFR in AVA neurons were imaged in the area of the proximal section on the AVA neurites (which usually receives ASH inputs). Light gray shading indicates 20 s period of exposure to a $1 \mathrm{mM} \mathrm{NaCl}$ stimulus, beginning at $t=3 \mathrm{~s}$. The first $3 \mathrm{~s}$ of the recording was averaged to serve as the baseline fluorescence, $F_{0}$. Green box indicates a window of $10 \mathrm{~s}$ after neural response to stimulation reached a relative steady state, for which the fluorescence change $(\Delta \mathrm{F})$ was averaged and presented in the bar graphs. $* * * P<0.001$, ANOVA with Bonferroni correction. Light colored shading around each curve and the error bars on bar graphs indicate SEM. $n=5-7$ for each strain. 

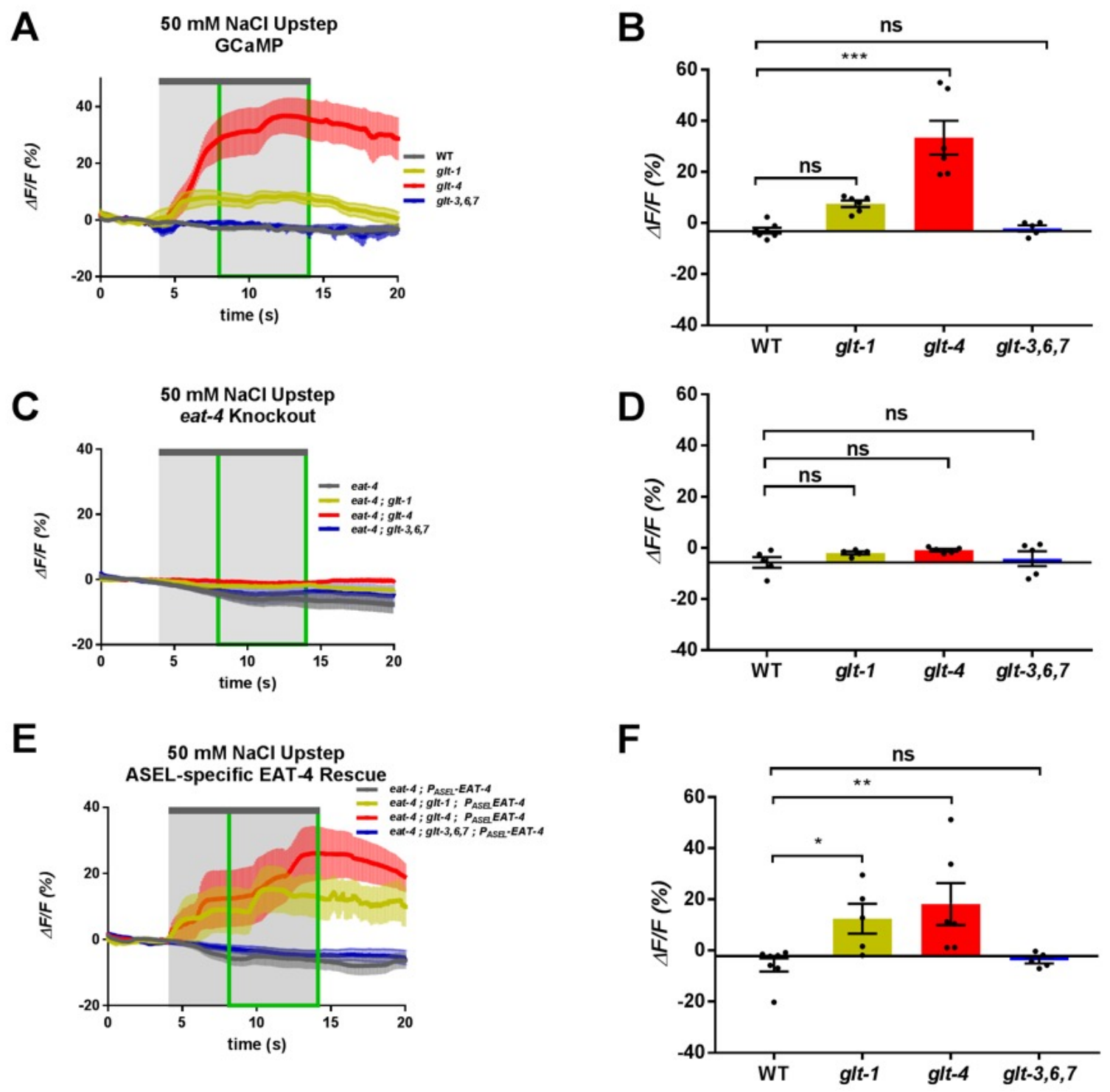

Figure 12. AVA spillover responses to $50 \mathrm{mM} \mathrm{NaCl}$ increase are also affected by proximal GluT KO and depend on Glu release from ASEL (similar to responses to $1 \mathrm{mM} \mathrm{NaCl}$ increase)

A; B) Proximal GluT KOs cause AVA to respond to $50 \mathrm{mM} \mathrm{NaCl}$ concentrations.

Low-salt-induced calcium responses in AVA neurons of WT and GluT KO animals. Average traces of changes in GCaMP3 fluorescence and averaged steady-state responses to a $50 \mathrm{mM} \mathrm{NaCl}$ stimulus are analyzed similarly to those in Figure 11A, B. ${ }^{* * *} P<0.001, * P<0.05$. ANOVA with Bonferroni correction. $\mathrm{n}=5$ for each strain.

C; D) Putative spillover onto AVA in response to $50 \mathrm{mM} \mathrm{NaCl}$ stimulation is eliminated in proximal GluT KO mutants in a mutant eat-4 background. $50 \mathrm{mM} \mathrm{NaCl}$-induced changes in Glu concentrations in nerve ring processes of AVA neurons in WT and GluT KO animals with LOF mutations of vesicular GluT eat-4. Average traces of changes in GCaMP3 fluorescence and averaged steady-state responses to $\mathrm{NaCl}$ stimulation are analyzed similarly to those in Fig 11A, B. ${ }^{* * *} P<0.001$. ANOVA with Bonferroni correction. Light colored shading around curves and the error bars on bar graphs indicate SEM. $n=5$ for each strain. 
Figure 12 (continued). E; F) ASEL-specific Glu release is sufficient for restoring putative spillover onto AVA in response to $50 \mathrm{mM} \mathrm{NaCl}$ stimulation. $50 \mathrm{mM} \mathrm{NaCl}$-induced changes in Glu concentrations in nerve ring processes of AVA neurons in WT and GluT KO animals with functional EAT-4 expressed only in ASEL neurons. Average traces of changes in GCaMP3 fluorescence and averaged steady-state responses to $\mathrm{NaCl}$ stimulation are analyzed similarly to those in Fig 11A, B. *** $P<0.001$. ANOVA with Bonferroni correction. Light colored shading around curves and the error bars on bar graphs indicate SEM. $n=5-7$ for each strain.
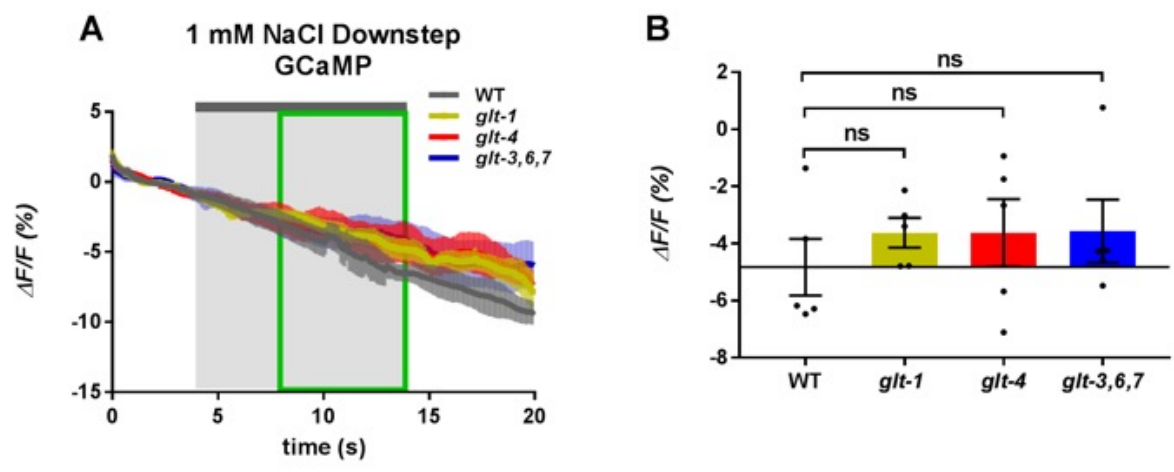

Figure 13. A 1 mM NaCl decrease does not stimulate AVA neurons of GluT KO animals. NaCl-induced changes in Glu concentrations in nerve ring processes of AVA neurons in WT and GluT KO animals. Average traces of changes in GCaMP3 fluorescence and averaged steady-state responses to a $1 \mathrm{mM} \mathrm{NaCl}$ decrease are analyzed similarly to those in Figure $11 \mathrm{~A}, \mathrm{~B}$. ${ }^{* * *} P<0.001$, ANOVA with Bonferroni correction. $\mathrm{n}=5$ for each strain.

We again verified that the aberrant somatic $\mathrm{Ca}^{2+}$ responses from GCaMP recordings are correlated with perisynaptic increases in Glu levels, as recorded with iGluSnFR in AVA neurites.

We observed a moderate increase in Glu concentrations in AVA synapses in response to stimulation of ASEL with a $1 \mathrm{mM} \mathrm{NaCl}$ upstep in glt-1 mutant animals (with a smaller effect in glt-3;6;7 mutant animals; glt-4 mutant animals were not tested for logistical reasons) (Figure 11C, D). These observations suggest that in the absence of proximal GluTs, Glu released by circuits sensitive to a small upstep in $\mathrm{NaCl}$ concentrations now reach AVA.

We also wanted to ascertain the origin of this aberrant AVA response and pinpoint the neurons responsible for this instance of Glu release. To do so, we first examined the effect of eliminating the major vGluT, EAT-4, from all cells in the nematode. We recorded AVA responses of glt mutants with eat-4 loss-of-function and found that the aberrant AVA responses 
to $1 \mathrm{mM} \mathrm{NaCl}$ upstep in glt-1 and glt-4 mutant animals are almost completely eliminated (Figure 14A, B). Similar results were obtained for ASEL stimulation with a 50mM NaCl upstep (Figure 12C, D). To confirm that ASEL is the origin of the Glu signal responsible for AVA responses to small $\mathrm{NaCl}$ upstep in glt- $1 \mathrm{KO}$ and glt-4 $\mathrm{KO}$ animals, we performed salt stimulation experiments in glt mutants containing ASEL-specific eat-4 rescue (using the ASEL -specific promoter $P_{g c y-7}$ to drive eat-4 expression, Figure 14C, D). We observe that restoring Glu release only in ASEL was sufficient in restoring the aberrant AVA response to small $\mathrm{NaCl}$ upstep in proximal glt mutants. Similar results were obtained for ASEL stimulation with a $50 \mathrm{mM} \mathrm{NaCl}$ upstep (Figure 12E, F). Neither $1 \mathrm{mM} \mathrm{NaCl}$ downstep (Figure 14A, B) nor $1 \mathrm{M}$ glycerol stimulation (Figure 15C, D) triggered a response in AVA when EAT-4 is expressed only in ASEL, confirming the specificity of the aberrant AVA response. Additionally, we ruled out the possibility of $\mathrm{ASH}$ responding to small $\mathrm{NaCl}$ upstep, since restoring EAT-4 only to ASH did not restore AVA responses to $1 \mathrm{mM} \mathrm{NaCl}$ upstep (Figure 16). 

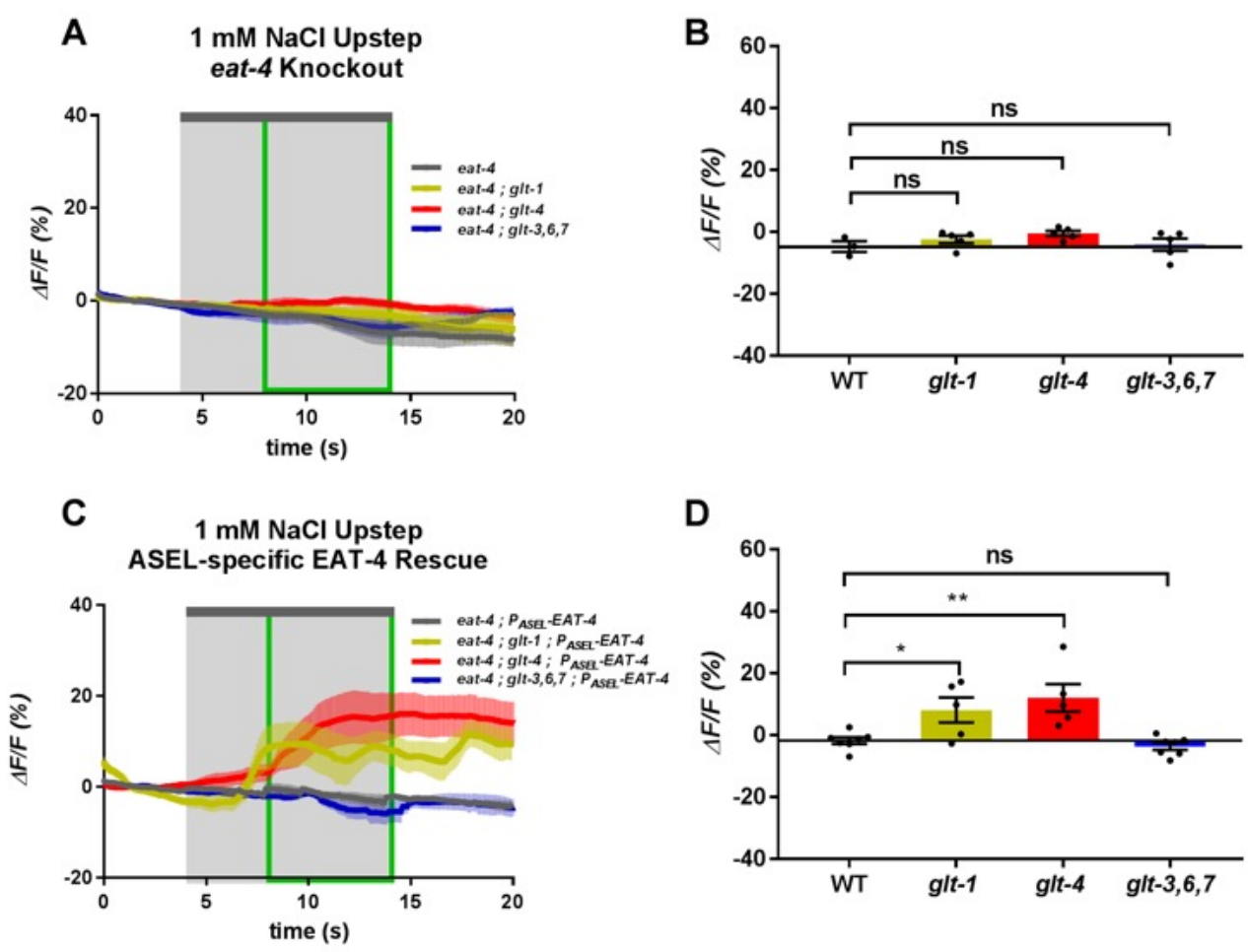

Figure 14. The effect of proximal GluT KO on AVA response to ASEL stimulation is mediated by spillover of Glu released from ASEL.

A, B) Ectopic responses of AVA to stimulation of ASEL depend (at least primarily) on the vGluT EAT-4. $1 \mathrm{mM} \mathrm{NaCl}$-induced changes in GCaMP3 fluorescence of AVA neurons in WT and GluT KO animals with LOF mutations of vGluT eat-4. Average traces of changes in GCaMP3 fluorescence and averaged steady-state responses to $\mathrm{NaCl}$ stimulation are analyzed similarly to those in Fig 11A, B. *** $P<0.001$. ANOVA with Bonferroni correction. Light colored shading around curves and the error bars on bar graphs indicate SEM. $n=5$ for each strain.

C, D) ASEL-specific Glu release is sufficient for restoring putative spillover onto AVA in response to $1 \mathrm{mM}$ NaCl stimulation.

$1 \mathrm{mM} \mathrm{NaCl}$-induced changes in Glu concentrations in nerve ring processes of AVA neurons in WT and GluT KO animals with functional EAT-4 expressed only in ASEL neurons. Average traces of changes in GCaMP3

fluorescence and averaged steady-state responses to $\mathrm{NaCl}$ stimulation are analyzed similarly to those in Fig 11A, B. $* * * P<0.001$. ANOVA with Bonferroni correction. Light colored shading around curves and the error bars on bar graphs indicate SEM. $\mathrm{n}=5-7$ for each strain. 

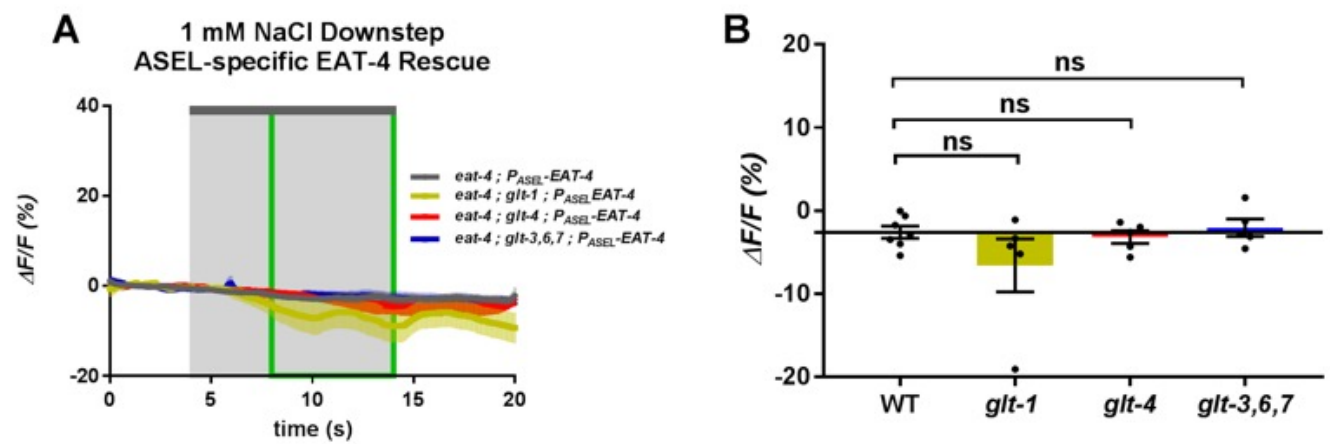

C
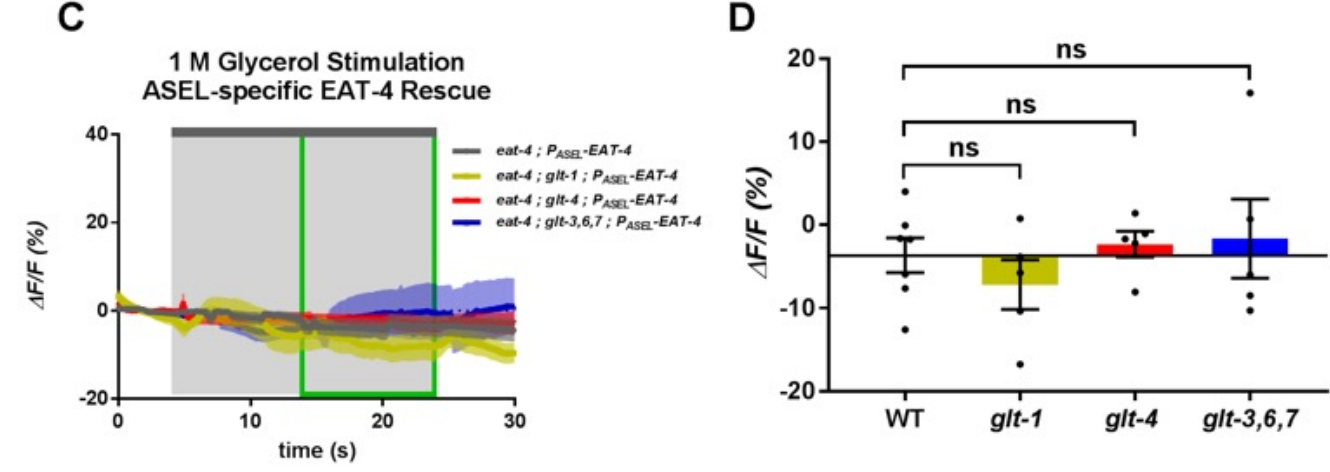

Figure 15. ASEL-specific eat-4 rescues respond to neither $1 \mathrm{mM}$ decrease in $\mathrm{NaCl}$ concentration nor $1 \mathrm{M}$ glycerol stimulation. $1 \mathrm{mM} \mathrm{NaCl}$ decrease (A) and $1 \mathrm{M}$ glycerol stimulation (C) fail to induce changes in Glu concentrations in nerve ring processes of AVA neurons in WT and GluT KO animals with functional EAT-4 expressed only in ASEL neurons. Average traces of changes in GCaMP3 fluorescence and averaged steady-state responses to $\mathrm{NaCl}$ stimulation (B and D) are analyzed similarly to those in Fig $11 \mathrm{~A}, \mathrm{~B}$; and Fig5 A, B respectively. ${ }^{* * *} P<0.001$. ANOVA with Bonferroni correction. Light colored shading around curves and the error bars on bar graphs indicate SEM. $n=5-7$ for each strain.
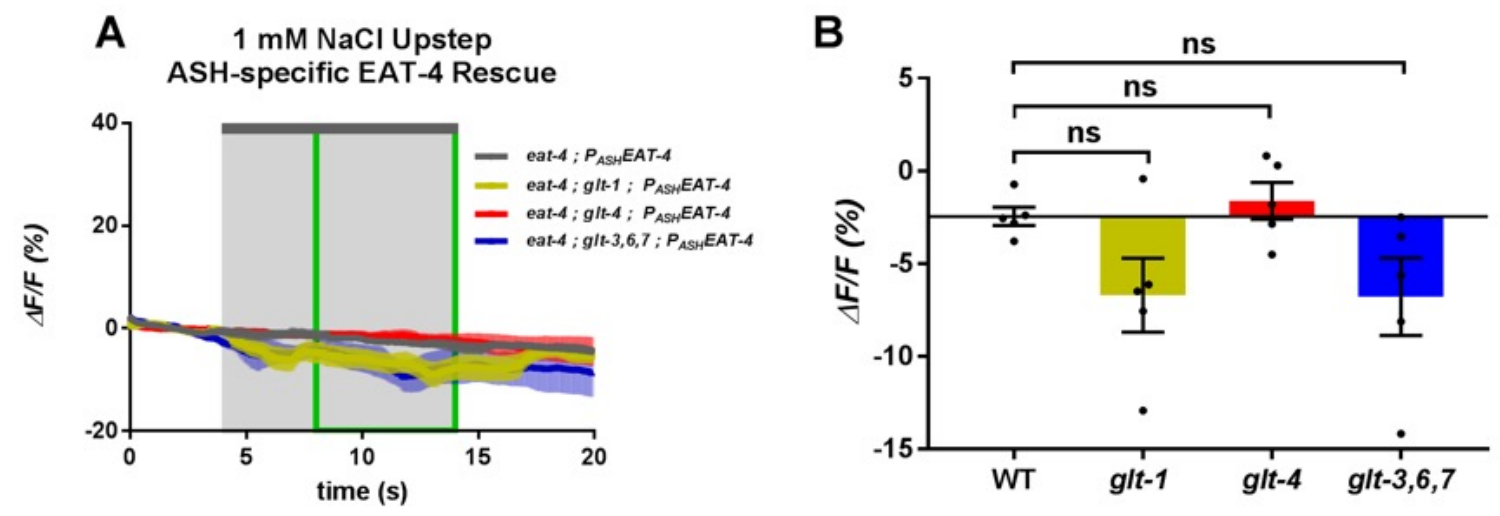

Figure 16. ASH-specific rescue of Glu release in proximal GluT KO animals in a mutant eat-4 background fails to restore putative spillover onto AVA in response to $1 \mathrm{mM} \mathrm{NaCl}$ stimulation. $1 \mathrm{mM} \mathrm{NaCl}$-induced changes in Glu concentrations in nerve ring processes of AVA neurons in WT and GluT KO animals with LOF mutations of vesicular GluT eat-4. Extrachromosomal constructs allow for ASH-specific expression of wild-type eat-4. Average traces of changes in GCaMP3 fluorescence and averaged steady-state responses to $\mathrm{NaCl}$ stimulation are analyzed similarly to those in Fig 11A, B. ${ }^{* * *} P<0.001$, ANOVA with Bonferroni correction. Light colored shading around curves and the error bars on bar graphs indicate SEM. $n=5$ for each strain. 
Altogether, these observations show that in the absence of proximal GluTs, Glu released from ASEL (in response to animal stimulation with a small $\mathrm{NaCl}$ upstep) escapes the ASEL -> AIA/AIB/AIY circuit and spills over to the ASH -> AVA/AVD/AVE circuit. According to this view, Glu spilled over from ASEL-AIA/AIB/AIY synapses now generates an abnormal avoidance response to a normally chemoattractive stimulant. These observations also suggest that in the WT, proximal GluTs are positioned to have a privileged effect on Glu released by ASEL and prevent its spillover beyond its designated postsynaptic targets, while distal GluT preferentially clears Glu released from ASH. Therefore, different GluTs exhibit specialized or privileged functional roles in different synapses.

\subsubsection{Proximal and distal GluTs are at least partially non-interchangeable, suggesting they are endowed with distinct functional properties.}

Given the difference in effects of proximal and distal GluT KOs, we wonder if they possess different clearance functions as determined by their innate physiological properties. One way to begin to address this question is to swap the expression of these GluTs, i.e., to ectopically express one transporter instead of the other. We prepared transgenic constructs, with the promoter of the proximal GluT glt- 1 is driving the expression of the distal GluT glt-3 $\left(P_{\text {glt- } 1:: g l t-}\right.$

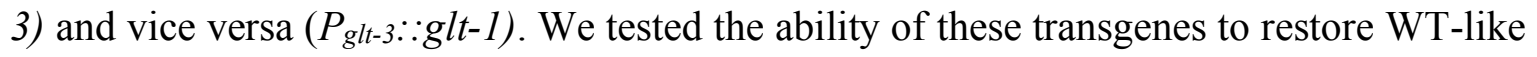
responses when the original GluT is missing. We asked: Can $P_{g l t-1} \because g l t-3$ transgene restore WTlike responses to glt- 1 mutant animals? And can a $P_{g l t-3}:: g l t-1$ transgene restore WT-like responses to glt-3 mutant animals? A caveat of rescue by extrachromosomal transgene is the non-native expression level: standard extrachromosomal transgene expression typically result in overexpression. Therefore, it is possible that suboptimal function of a given transporter for a 
specific role might be masked by its transgenic overexpression. This issue could potentially be resolved through single-copy transgene integration technology (currently beyond the scope of this study). However, if a given GluT cannot replace another one even with the current overexpression, this will indicate that the two transporters are not functionally interchangeable. As a control (especially since these transgenes are based on cDNA, not fully native genomic constructs), we tested the abilities of $P_{g l t-1:}: g l t-1$ and $P_{g l t-3:: g l t-3}$ transgenes to restore WT-like responses to mutants animals where these respective genes are missing. We tested responses to chemoattractive salt stimulation in $g l t-1$ animals rescued with $P_{\text {glt-1 }}: \because g l t-1$, or responses to high osmolarity nociceptive stimulation in $g l t-3$ animals rescued with $P_{g l t-3}:: g l t-3$. We observe that restoration of $g l t-3$ expression under its native promoter (by expressing the $P_{g l t-3:}: g l t-3$ transgene in $g l t-3 \mathrm{KO}$ animals) restored WT-like AVA responses to nociceptive stimulation, eliminating the exaggerated AVA responses typically seen in these animals (Figure 17A, B). However, expressing glt- 1 ectopically where $g l t-3$ is normally expressed (by expressing the $P_{g l t-3:: g l t-1}$ transgene in $g l t-3 \mathrm{KO}$ animals) failed to restore WT-like responses of AVA to nociceptive stimulation (also seen in Figure 17 A, B). Therefore, even overexpression of glt-1 in places where $g l t-3$ is normally expressed cannot compensate for loss of glt-3 (despite glt-1 cDNA encoding a fully functional GLT-1, as shown by the next experiment). 

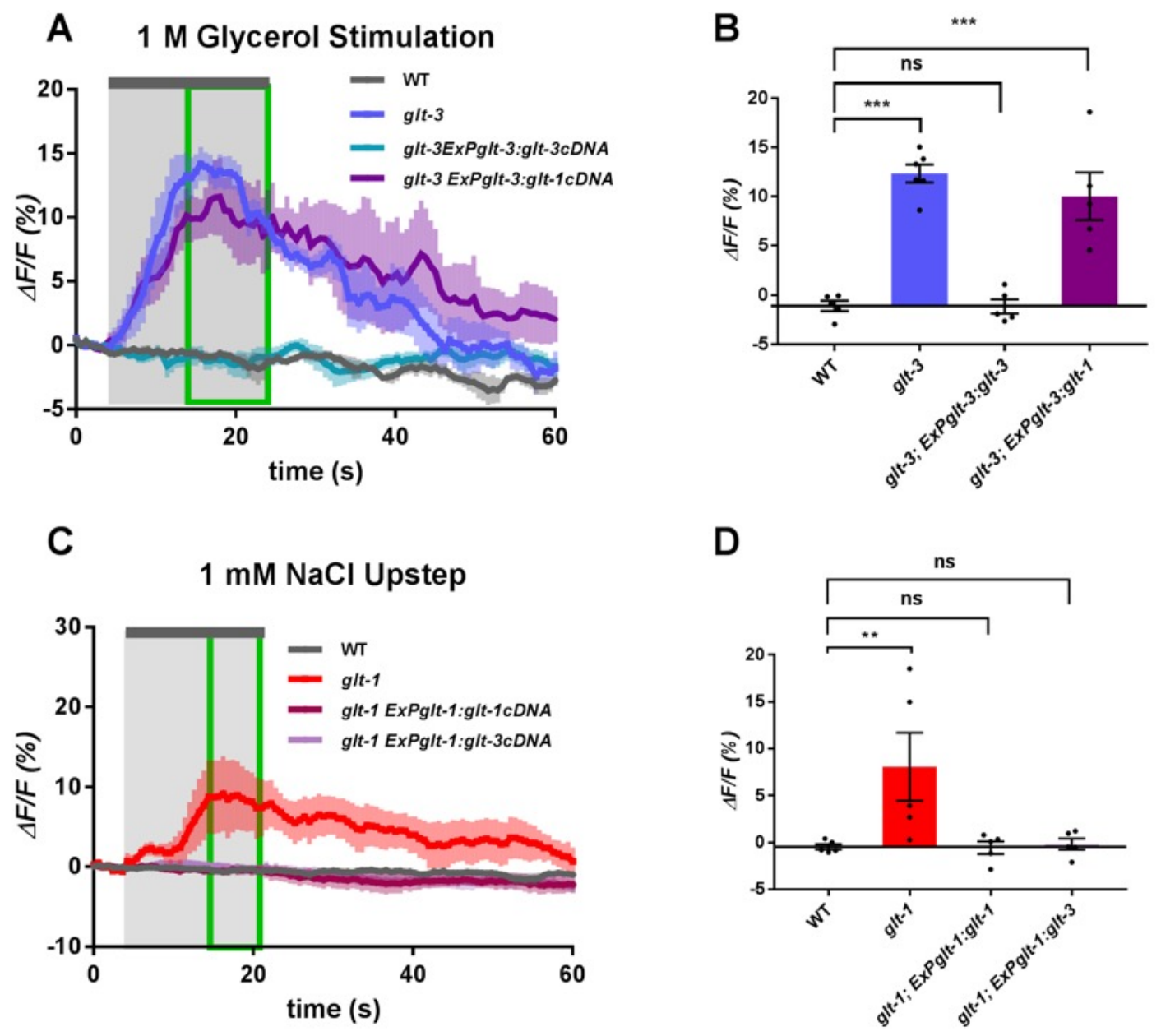

Figure 17. Distal and proximal GluTs are partially distinct in their molecular function.

A, B) The exaggerated response of AVA to ASH stimulation in distal GluT KO animals can be eliminated by expressing a distal GluT at its native location. The exaggerated response of AVA to ASH stimulation in distal GluT KO animals can be rescued (i.e., reverted back to WT-like response) by rescuing expression of a distal GluT in its native location, but not by expressing a proximal GluT in this distal location. glt-3 mutants expressing $P_{g l t-3}: g l t-1$ constructs still retain strong AVA response upon ASH stimulation, indicating that GLT-1 overexpression cannot rescue glt-3 KO, and GLT-1 \& GLT-3 are not interchangeable. The first $4 \mathrm{~s}$ of the recording was averaged to serve as the baseline fluorescence, $\mathrm{F}_{0}$. Green box indicates a window of $10 \mathrm{~s}$ after neural response to stimulation reached a relative steady state, for which the fluorescence change $(\Delta \mathrm{F})$ was averaged and presented in the bar graphs. $* * * P<0.001$, ANOVA with Bonferroni correction. Light colored shading around each curve and the error bars on bar graphs indicate SEM. $\mathrm{n}=5-6$ for each strain.

C,D) The spillover of Glu to AVA neurites upon ASEL stimulation in proximal GluT KO animals can be eliminated by expressing either proximal or distal GluT at proximal locations. The spillover of Glu to AVA neurites upon ASEL stimulation in proximal GluT KO animals can be rescued (i.e., reverted back to WT-like response) by reinstating the expression of this proximal GluT, but also by overexpressing a distal GluT in this proximal location. The first $4 \mathrm{~s}$ of the recording was averaged to serve as the baseline fluorescence, $\mathrm{F}_{0}$. Green box indicates a window of $6 \mathrm{~s}$ after neural response to stimulation reached a relative steady state, for which the fluorescence change $(\Delta \mathrm{F})$ was averaged and presented in the bar graphs. $* * * P<0.001$, ANOVA with Bonferroni correction. Light colored shading around each curve and the error bars on bar graphs indicate SEM. $n$ $=5$ for each strain. 
We also examined the ability of ectopically expressed $g l t-3$ to compensate for the loss of glt-1. We verified that restoration of $g l t-1$ expression under its native promoter (by expressing the $P_{g l t}$ 1::glt-1 transgene in $g l t-1 \mathrm{KO}$ animals) restored WT-like non-response of AVA to ASEL stimulation, eliminating the aberrant AVA responses typically seen in $g l t-1 \underline{\mathrm{KO}}$ animals (Figure 17 C, D). However, unlike the exaggerated responses in the ASH->AVA circuit, ectopic overexpression of $g l t-3$ in the locations where $g l t-1$ is normally expressed (by expressing the $P_{g l t-1}: \because g l t-3$ transgene in $g l t-1 \mathrm{KO}$ animals) managed to eliminate aberrant AVA responses to ASEL stimulation. As stated above, at this stage it is not clear if this rescue and functional overlap is due to the ability of GLT-3 overexpression to mask some deficiencies in GLT-1 -like functions, or due to actual overlap in properties. We also verified that expression of these transgenes does not modify other responses (for example, overexpressing proximal or distal GluTs does not modify lack of exaggerated AVA responses to ASH stimulation in proximal GluT KO, or the lack of aberrant AVA responses to ASEL stimulation of distal GluT KO, Figure 18). Taken together, the inability of ectopically-expressed GLT-1 (even when overexpressed) to compensate for the loss of GLT-3 indicates that these transporters are at least partially non-interchangeable, and therefore functionally distinguishable. These transgenic constructs contained SL2::mCherry fused 3' to the GluT ORF, which we used to reaffirm our previous finding on the location of expression of these transporters ${ }^{35}$. Our observations prompted us to look more closely at the significance of the location of expression of these GluTs. 

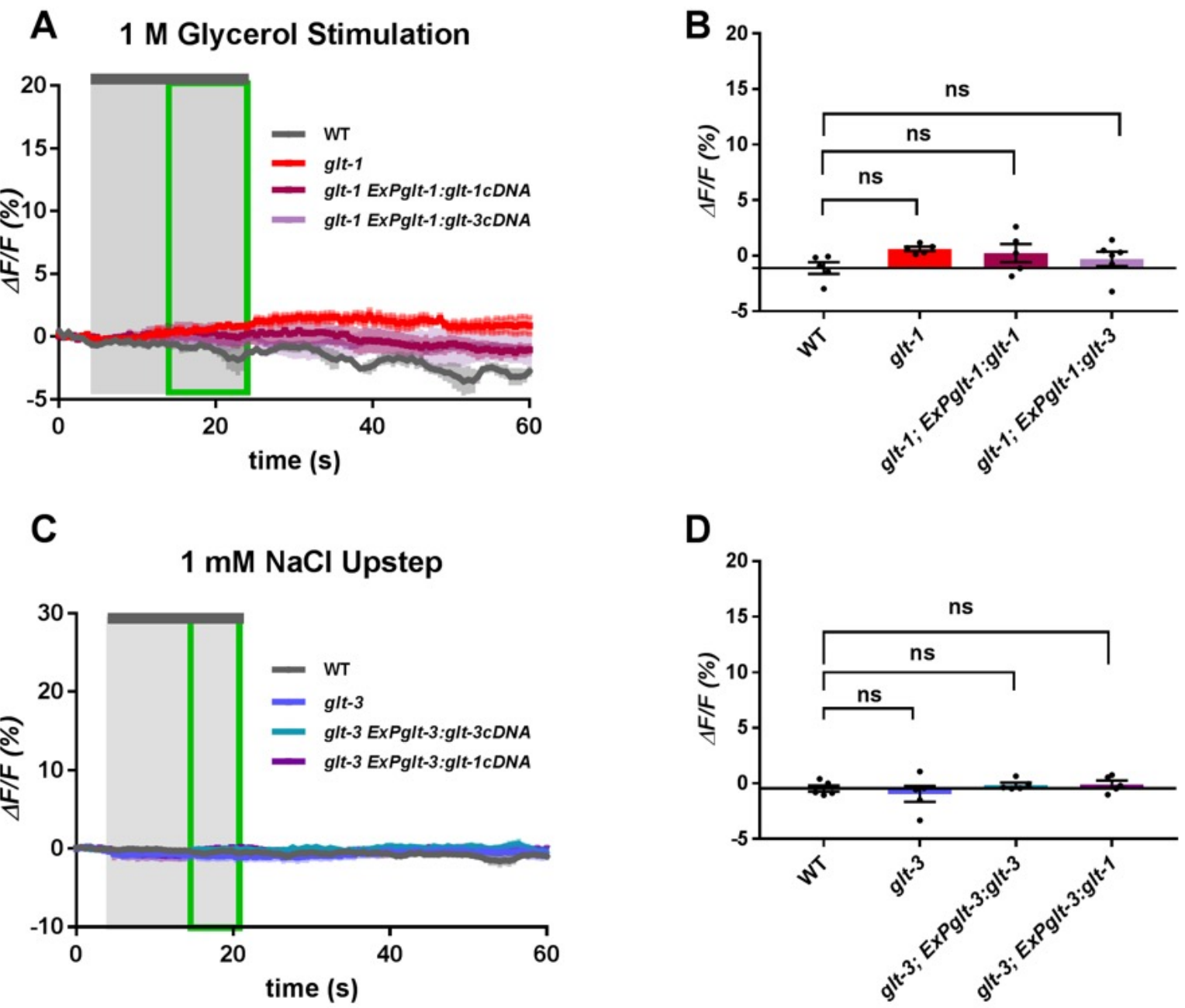

Figure 18. glt-1 mutant animals overexpressing GluTs do not display exaggerated AVA responses to ASH stimulation, nor do glt-3 mutant animals display aberrant AVA responses to ASEL stimulation.

A, B) Animals expressing endogenous GLT-3 do not display exaggerated response of AVA to ASH stimulation in distal GluT KO animals. glt-1 mutants expressing Pglt-1:glt-1 and Pglt-1:glt-3 constructs retain a WT-like AVA response upon ASH stimulation. Exaggerated AVA responses to ASH stimulation are dependent on the absence of functional GLT-3, rather than the absence of functional GLT-1 or transgenic GluT rescue in proximal locations. The first $4 \mathrm{~s}$ of the recording was averaged to serve as the baseline fluorescence, $F_{0}$. Green box indicates a window of $10 \mathrm{~s}$ after neural response to stimulation reached a relative steady state, for which the fluorescence change $(\Delta \mathrm{F})$ was averaged and presented in the bar graphs. $* * * P<0.001$, ANOVA with Bonferroni correction. Light colored shading around each curve and the error bars on bar graphs indicate SEM. $\mathrm{n}=5-6$ for each strain. 


\subsubsection{Differential localization of ASH and ASE synapses in the nerve ring might contribute to the differential effect of proximal and distal Glu clearance.}

Based on the data obtained from our recordings of AVA neuron responses, it appears that while distal GluTs glt-3, 6, \& 7 possess a privileged role in clearance of inner nerve rim synapses of the nociceptive ASH -> AVA/AVD/AVE circuit, proximal GluTs glt-1 \& 4 may have priority over clearance of outer nerve rim synapses (such as the circuit adjacent to ASH, the ASE -> AIA/AIB/AIY chemotaxis circuit). We looked into existing anatomical data on the synaptic locations of these two circuits: the nematode connectome was established by utilizing EM images of coronal sections and reconstruction data (initially published as "The Mind of the Worm") ${ }^{44}$. This data was later developed by D. Hall's and S. Emmons's labs in the form of the comprehensive web resources available at WormAtlas.org, WormWiring.org, and CytoShow.org ${ }^{7,45}$. The data is available as a collection of fully annotated consecutive slices and as a complete record of the location and identity of the synapses, as well as 3D reconstruction of all neurons ${ }^{32,46}$. We used these web resources to locate the synapses relevant to our study. We find that the neuronal processes of the two circuits we study are indeed adjacent, as they track rather closely in the nerve ring. Furthermore, the ASE -> AIA/AIB/AIY and ASH -> AVA/AVD/AVE synapses, as identified in the Worm Connectome project, are frequently found in close proximity to each other. Figure 19A shows slice \#87 of the EM series, which depicts this proximity of ASE -> AIB/AIY synapses (cell processes outlined in red) and ASH -> AVD synapses (cell processes outlined in blue), with the synapse of the latter gradually expanding to add AVA to the left of AVD (fully visible in slice \#91). Overall, the synapses of the two circuits appear favorably positioned for Glu spillover to occur. 


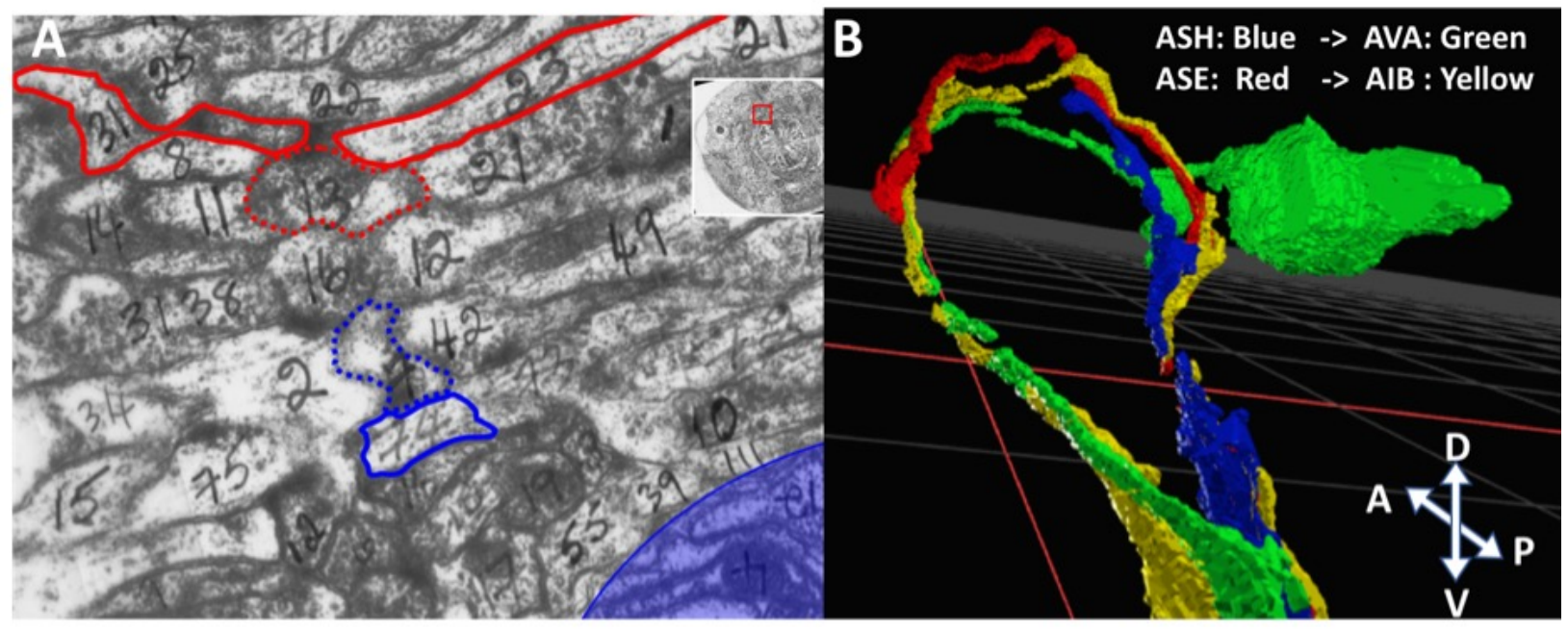

Figure 19. Analysis of EM data from the $C$. elegans nerve ring suggests that synapses of different circuits are found in great proximity to each other.

A) This figure is based on a section of the original EM image from White et al's "The Mind of the Worm" (through WormAtlas.org and WormWiring.org). The image corresponds to slice \# 87, where penciled numbers mark cell assignments. Our assignment of synapses is based on analysis by the Hall and Emmons labs, as appearing in WormWiring.org . Cell outlines are tentatively marked by us with limited accuracy (based on our best estimate from this image, and the images of adjacent slices). Cells of the avoidance circuit (ASH -> AVA/AVD/AVE) are marked with a blue outline; Cells of the salt chemoattraction circuit (ASE -> AIA/AIB/AIY) are marked with a red outline. In both cases, presynaptic terminals are marked in a dashed line. The image shows a chemical synapse between presynaptic ASHL (cell \#7) and postsynaptic AVDL (cell \#74) and AVBL (cell \#2) (although AVAL is not seen here, in the immediately following slices AVAL joins this synapse, as it squeezes between AVDL and AVBL). Another chemical synapse is formed between presynaptic ASEL (cell \#13) and postsynaptic AIYL (cell \#31) and AIBL (cell \#23). Blue shade at the bottom right indicates the pseudocoelomic area between the nerve ring and the pharynx containing the end of the muscle arms and GLR cells. The insert in the upper right shows a zoom-out view of this area, with a red box corresponding approximately to the enlarged area. Key to cell numbers is based on cytoshow.org . Note: these numbers are only part of the full cell designation, so some numbers appear more than once. Key: 1 AIAL ; 2 - AVBL ; 3 (should have been 18) - URADL ; 4 (written upside down on lower right) DLV4 DBW muscle arm ; 6 - URBL ; 7 - ASHL ; 8 - AIZL ; 9 - ; 10 - ; 11 - ADFL ; 12 (between red and blue marked synapses) - AWAL ; 12 (lower center part of image) - ADAL ; 12 (written upside down, lower right) - DLV12 DBW muscle arm ; 13 ASEL ; 14 - RIR ; 15 (lower left) - RID ; 15 - OLQDL ; 16 (center, between red and blue marked synapses) - AWBL ; 16 (lower center) - URYDL ; 17 - IL1DL ; 19 - IL2DL ; 21 - ASKL ; 22 - AWCL ; 23 - AIBL ; 25 - AFDL ; 31 (top left, outlined in red) - AIYL ; 31 (center left, no outline) - PVPL ; 34 - PVCR ; 38 - AVHR ; 39 - PVNL ; 42 - HSNL ; 49 - HSNR ; 55 - PVR ; 71 - AINL ; 72 - AVHL ; 73 AVJL ; 74 - AVDL ; 75 - AVJR ; 111 - PVNR

B) 3D reconstruction of the relevant neurons from WormWiring.org. Color key for the different neurons is at the upper right. In this projection image, Anterior is left and diagonal up, Posterior is right and diagonal down, Dorsal is vertical up, Ventral is vertical down. ASE->AIB contacts are radially lateral to the ASH->AVA contacts.

We expanded our examination to additional slices where these synapses are found (\# 80-130),

using WormWiring.org and CytoShow.org (Figure 20 presents annotation of slices \# 79, 84, 94, and 99 as examples). Together with WormWiring 3D reconstruction (Figure 19B), we find that the ASE -> AIA/AIB/AIY synapses are consistently found closer to (and seem to have synaptic 
vesicles released toward-) the outer rim of the nerve ring (closer to the muscle and surrounding hypodermis). In contrast, the ASH -> AVA/AVD/AVE synapses are found closer to (and seem to release synaptic vesicles toward-) the inner rim of the nerve ring (closer to the pharynx). Both our previous GLT-1::GFP fusion studies ${ }^{35}$ and our new constructs (expressing $P_{\text {glt-1 }}: \because g l t-$ $1:: S L 2:: m$ Cherry) indicate that $g l t-1$ is heavily expressed in head muscles and in hypodermis, while Katz \& Shaham added that it is also expressed in cephalic sheath glia ${ }^{33}$ that wraps around the outer circumference of the nerve ring. The juxtaposition of the ASE -> AIA/AIB/AIY synapses and the glt-1 (proximal GluT) -expressing head muscles, hypodermis, and glia could therefore provide a basis for the privileged role of $g l t-1$ in clearing Glu released from ASE, and explain the susceptibility of $g l t-1 \mathrm{KO}$ animals to the putative spillover of Glu out of ASE -> AIA/AIB/AIY synapses (as evidenced by AVA stimulation by low $\mathrm{NaCl}$ concentrations in these animals, Figures $11 \&$ 14). 


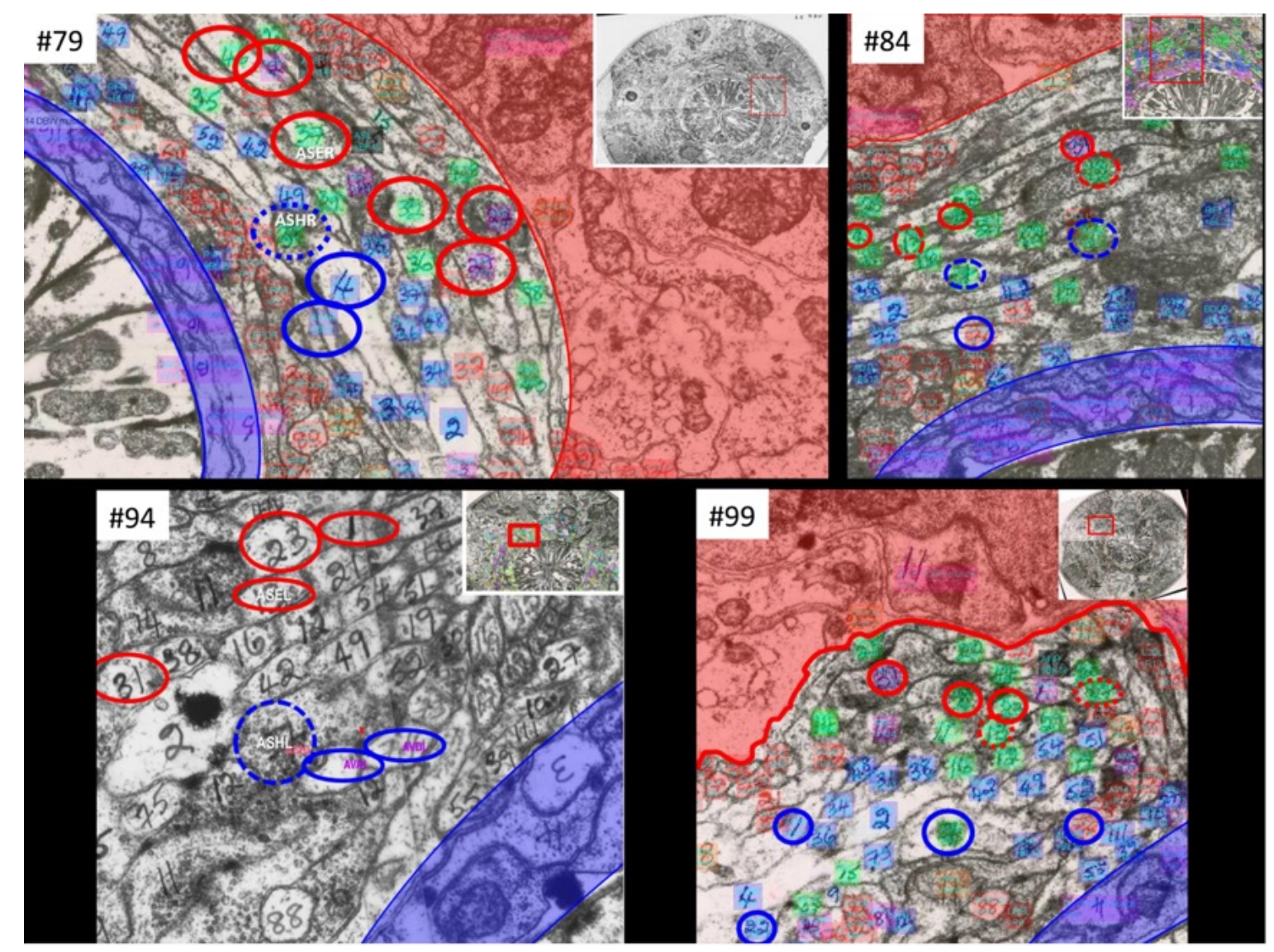

Figure 20. Analysis of EM data from the $C$. elegans nerve ring suggests that synapses of different circuits are found in great proximity to each other.

The figure is based on a section of the original EM image from White et al's "The Mind of the Worm" [White, 1986 \#1816] (through WormAtlas.org and WormWiring.org). The image corresponds to slice \# 87, where penciled numbers mark cell assignments. Our assignment of synapses is based on analysis by the Hall and Emmons labs, as appearing in WormWiring.org . Cell outlines are tentatively marked by us with limited accuracy (based on our best estimate from this image, and the images of adjacent slices). Cells of the avoidance circuit (ASH -> AVA/AVD/AVE) are marked with a blue outline; Cells of the salt chemoattraction circuit (ASE -> AIA/AIB/AIY) are marked with a red outline. In both cases, presynaptic terminals are marked in a dashed line. The image shows a chemical synapse between presynaptic ASHL (cell \#7) and postsynaptic AVDL (cell \#74) and AVBL (cell \#2) (although AVAL is not seen here, in the immediately following slices AVAL joins this synapse, as it squeezes between AVDL and AVBL). Another chemical synapse is formed between presynaptic ASEL (cell \#13) and postsynaptic AIYL (cell \#31) and AIBL (cell \#23). Blue shade at the bottom right indicates the pseudocoelomic area between the nerve ring and the pharynx containing the end of the muscle arms and GLR cells. The insert in the upper right shows a zoom-out view of this area, with a red box corresponding approximately to the enlarged area.

Key to cell numbers is based on cytoshow.org . Note: these numbers are only part of the full cell designation, so some numbers appear more than once. Key: 1 - AIAL ; 2 - AVBL ; 3 (should have been 18) - URADL ; 4 (written upside down on lower right) DLV4 DBW muscle arm ; 6 - URBL ; 7 - ASHL ; 8 - AIZL ; 9 - ; 10 - ; 11 - ADFL ; 12 (between red and blue marked synapses) - AWAL ; 12 (lower center part of image) - ADAL ; 12 (written upside down, lower right) - DLV12 DBW muscle arm ; 13 - ASEL ; 14 - RIR ; 15 (lower left) RID ; 15 - OLQDL ; 16 (center, between red and blue marked synapses) - AWBL ; 16 (lower center) URYDL ; 17 - IL1DL ; 19 - IL2DL ; 21 - ASKL ; 22 - AWCL ; 23 - AIBL ; 25 - AFDL ; 31 (top left, outlined in red) - AIYL ; 31 (center left, no outline) - PVPL ; 34 - PVCR ; 38 - AVHR ; 39 - PVNL ; 42 HSNL ; 49 - HSNR ; 55 - PVR ; 71 - AINL ; 72 - AVHL ; 73 - AVJL ; 74 - AVDL ; 75 - AVJR ; 111 PVNR 


\subsubsection{Motility of the head and pharynx is critical to preserving the fidelity of AVA synaptic}

activity. Although the privileged role of the proximal glt-1 on ASE -> AIA/AIB/AIY synapses

can be explained by the proximity of these synapses to the head muscles, hypodermis, and glia, it remains unclear how the distal GluTs (glt-3, glt-6, \& glt-7), expressed on the canal cell, might preferentially affect the ASH -> AVA/AVD/AVE synapses, which are closer to the pharynx. Since the canal cell is directly exposed to pseudocoelomic body fluids, distal GluTs are likely to regulate ambient Glu levels in body fluids. Ambient extracellular Glu concentrations in the vicinity of mammalian synapses are known to affect synaptic Glu clearance by providing a "clean slate" for spontaneous Glu diffusion out of the synapse ${ }^{47-49}$. Interestingly, in the worm, nerve ring interstitial fluids are continuous with the pseudocoelomic fluid found in the space between the nerve ring and the isthmus of the pharynx (marked as blue shaded arch in Figures $19 \& 20)$. As described previously, solutes in the fluid of this region seem to have unfettered access to the inner rim of the nerve ring; and the basal laminae that separate different tissues are fully permeable to neurotransmitters and other small molecules ${ }^{50}$. We therefore considered a possible link between the putative ability of distal GluTs to control ambient Glu concentration in pseudocoelomic body fluids and the location of AVA synapses closer to the inner rim of the nerve ring. Considering that our experiments thus far were performed on physically-restrained (but not pharmacologically-paralyzed) animals, we contemplated the possibility that the continuous pulsations of the pharynx and the increased mobility that nematodes show in the head region (causing the nerve ring to slide slightly back and forth on the isthmus, even in restrained animals), may facilitate perfusion of body fluids to the inner part of the nerve ring. Such perfusion can replace extracellular fluids rich in synaptically released Glu with fresh body fluids containing lower, ambient levels of Glu. If normal Glu clearance from the inner rim is aided by 
mechanical agitation and perfusion, then paralysis might increase ambient Glu levels, potentially hampering Glu diffusion from these synapses, especially under conditions of increased synaptic activity and reduced Glu clearance. We hypothesize that mechanical agitation of body fluids and perfusion of the inner rim of the nerve ring might underlie the privileged effect of distal GluTs on the ASH -> AVA synapses (as seen in Figures 6-8) and affect the clearance of Glu that reach AVA by spillover (Figures 10-11, \& 14). In line with this hypothesis, we recently saw that under conditions where Glu accumulation leads to nematode excitotoxicity ${ }^{51}$, the neurons most severely affected by neurodegeneration are not those that express the most GluRs, but those that form Glu synapses in the innermost face of the nerve ring ${ }^{52}$.

Additionally, we suspected that inhibition of pharyngeal pumping and animal motility may prevent access of fresh body fluids to the synapses (in both WT and GluT KO animals), and interfere with the activity of synapses that depend on it. To test this hypothesis we first used tetramisole ${ }^{53}$, which acts as a constitutive, desensitizing agonist of nicotinic AcetylCholine Receptors (nAChRs) and is routinely used to paralyze worms for GCaMP-based imaging of specific neurons ${ }^{54-56}$ or the whole nervous system ${ }^{57}$. However, we had some reservations about using tetramisole in our specific studies because of nAChR expression in $\mathrm{AVA}^{58,59}$, though spontaneous activity and indirect odor responses of AVA remain intact in the presence of tetramisole ${ }^{57,60}$. To demonstrate the effect of paralysis without disrupting neuronal activity, we also performed experiments using $\mathrm{BDM}^{61}$, which induces paralysis directly in the muscle by inhibiting myosin. Though BDM also has off-target effects, we speculate that similarity in effects seen when using either tetramisole or BDM are likely to arise from the common effect of paralysis. 
Prior to our paralysis experiments, we wanted to verify that the paralyzing agents (particularly tetramisole) does not modify the activity of the presynaptic neuron. Indeed, by using GCaMP expressed in ASH we verified that tetramisole did not diminish ASH activity (compare response during paralysis in Figure 21B to initial stimulation in 21A). We also verified that a paired stimulation separated by 10 minute recovery shows no diminution of AVA response to the second stimulus (Figure 22). We performed this analysis for both AVA response to ASH stimulation with glycerol and for ASE stimulation with low concentration $\mathrm{NaCl}$, confirming that the previously described AVA responses in the different GluT KOs (Figures 7 \& 11) are preserved in paired stimuli of non-paralyzed animals (compare first stimulation in Figure 22A \& 22C, and second stimulation in 22B and 22D).
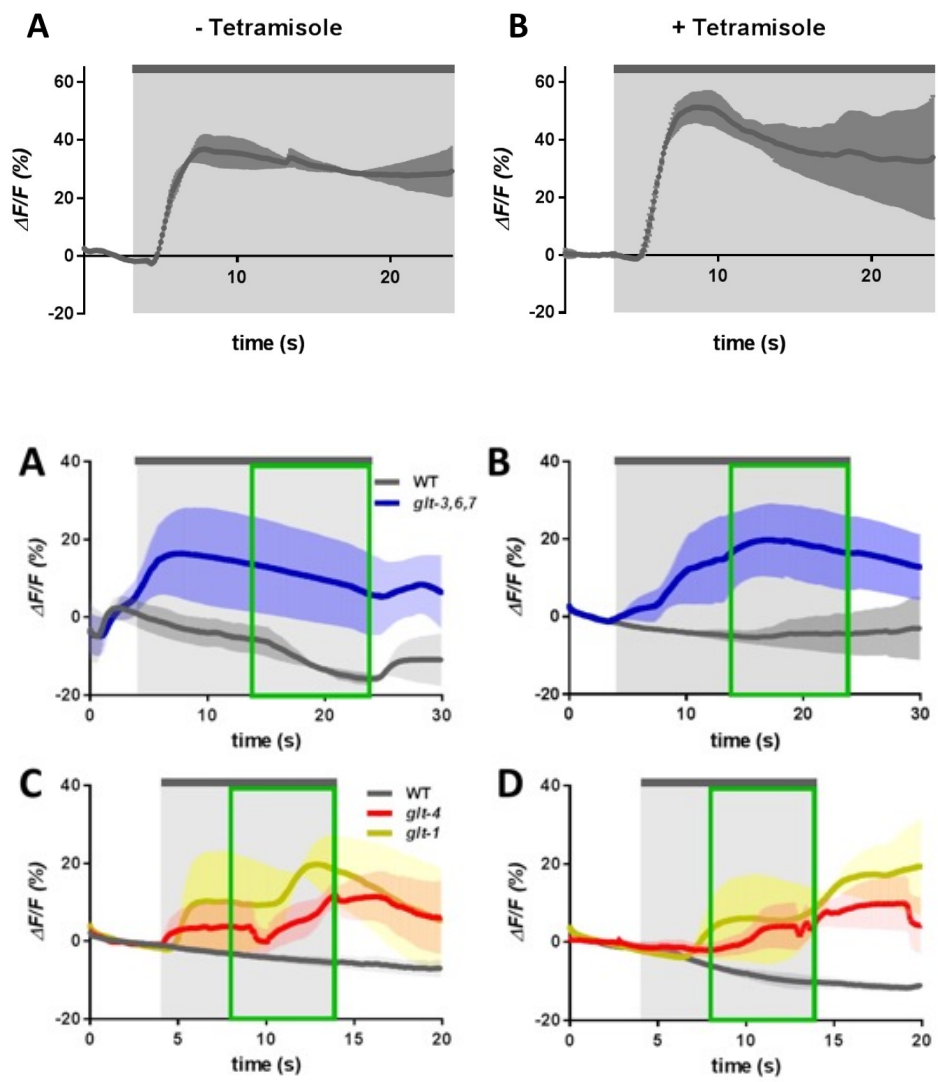

Figure 21. ASH responds to $1 \mathrm{M}$ glycerol before and during paralysis with $2 \mathrm{mM}$ tetramisole.

WT animals expressing GCaMP in AVA neurons were imaged before (A) and during paralysis (B) induced by $2 \mathrm{mM}$ tetramisole. Light gray shading in $\mathbf{A}$ and $\mathbf{B}$ indicates the $20 \mathrm{~s}$ period of stimulation with $1 \mathrm{M}$ glycerol, beginning at $t=4 \mathrm{~s}$. Light colored shading around each curve indicate SEM. $\mathrm{n}=2$.

Figure 22. Sequential stimulation of worms with $1 \mathrm{M}$ glycerol or $1 \mathrm{mM} \mathrm{NaCl}$ does diminish not exaggerated responses in AVA or putative spillover from ASE.

WT and GluT KO animals expressing GCaMP3 in AVA neurons were imaged. Light gray shading indicates the period of exposure to a $1 \mathrm{M}$ glycerol (A, B) or $1 \mathrm{mM} \mathrm{NaCl}$ stimulus (C,D). Stimulation begin at $t=4 \mathrm{~s}$. Light colored shading around each curve and the error bars on bar graphs indicate SEM. $n=2-4$ for each strain. 
To test the effect of paralysis on AVA responses to either ASH or ASE stimulation, we first applied stimulus under normal conditions, establishing the normal response in AVA in the specific animal. We then paralyzed the worm anterior section with either tetramisole or BDM in absence of chemical stimulation for 10 minutes. Finally, we exposed the paralyzed animal to the same stimulus a second time. We found that paralysis with tetramisole eliminated the exaggerated response of AVA to ASH stimulation in $g l t-3$, glt-6, glt-7 KO mutants (Figure 23AC). Similarly, tetramisole-mediated paralysis abolished the response of AVA to ASEL stimulation in $g l t-1$ and $g l t-4$ mutants (Figure 23D-F). We observed similar effects when worms were paralyzed with BDM (Figure 24), suggesting the effects we see do not arise from the various side effects of the two drugs, but from the shared effect of paralysis. We reasoned that if paralysis halts perfusion-mediated Glu clearance around AVA, then normal spontaneous activity (without stimulation) may result in Glu accumulation in AVA synapses during prolonged incubation with the paralyzing agent. In support of this hypothesis, we find GCaMP fluorescence in AVA to increase shortly after the onset of exposure to either tetramisole (Figure 25A, B) or BDM (Figure 25C, D). However, we could not resolve the details of this effect with direct Glu measurements at this time, because the weak iGluSnFR signals in AVA neurites are not conducive to such protracted imaging. 

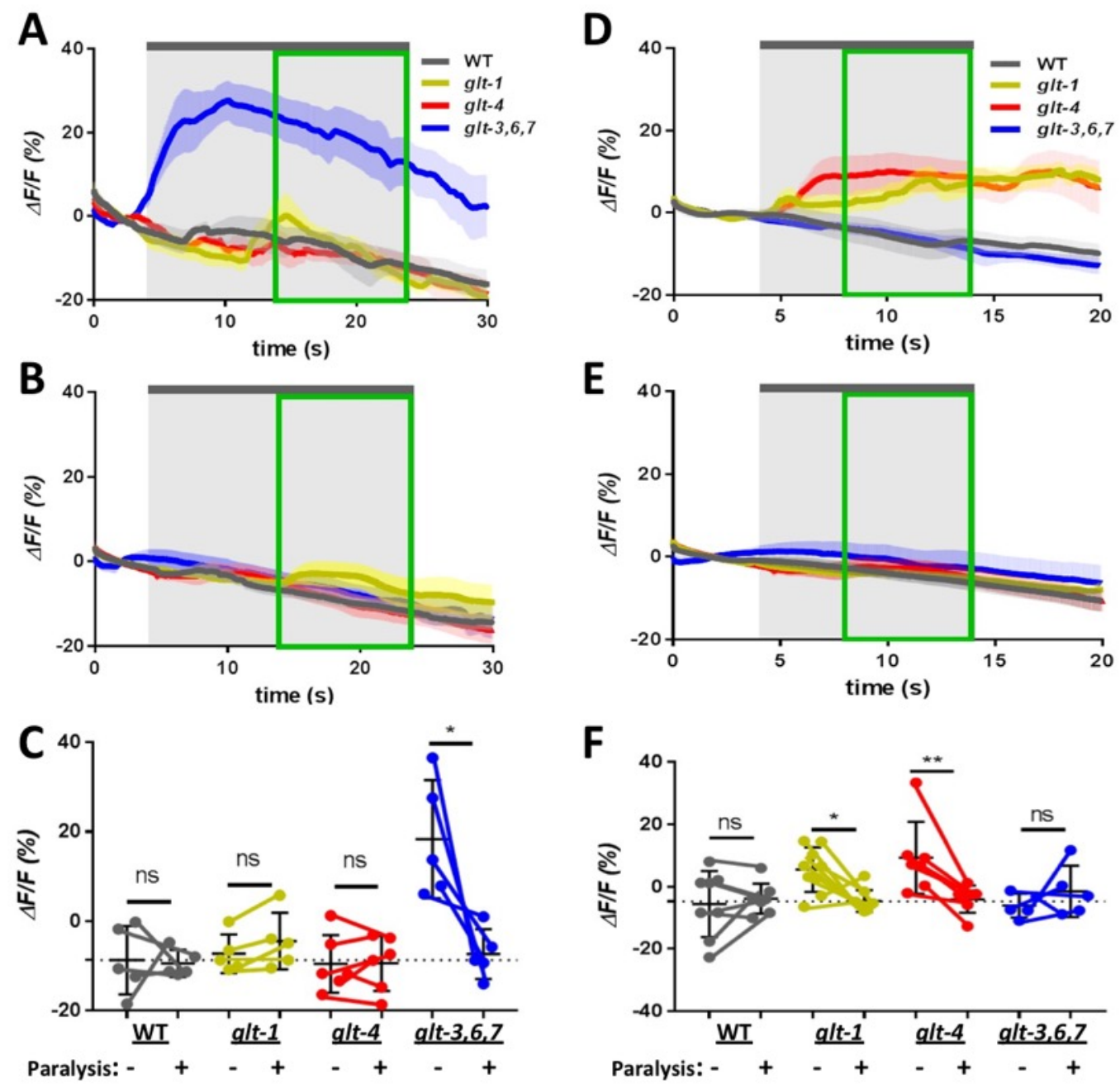

Figure 23. Paralysis eliminates or masks the exaggerated and ectopic postsynaptic responses of AVA.

A-C) Tetramisole-induced paralysis eliminates exaggerated responses in the ASH -> AVA synapse to 1 M glycerol. Head muscle and pharyngeal paralysis induced by exposure to tetramisole caused loss in AVA response of glt-3,6,7 mutants to stimulation by $1 \mathrm{M}$ glycerol. Changes in AVA GCaMP intensity in response to 1 $\mathrm{M}$ glycerol stimulation are shown before and during paralysis after $10 \mathrm{~min}$ treatment with tetramisole. Light grey indicates the exposure period of the worm to glycerol solution. Lightly colored shaded areas in (A) and (B) denote standard error. Average changes in fluorescence intensity during the $10 \mathrm{~s}$ window of stable state of stimulation before and after exposure to the paralytic agent are denoted in (C). Statistical significance is denoted with asterisks. $* P=0.0283$, ANOVA with Bonferroni correction. $\mathrm{n}=5-6$ per strain. Initial stimulation is shown in (A), while stimulation during paralysis is shown in (B).

D-F) Putative spillover from ASEL onto AVA is eliminated by tetramisole-induced head paralysis. Head muscle and pharyngeal paralysis induced by exposure to tetramisole caused loss in AVA response of glt-1 and glt-4 mutants to stimulation by $1 \mathrm{mM} \mathrm{NaCl}$. Changes in AVA GCaMP intensity in response to $1 \mathrm{mM} \mathrm{NaCl}$ stimulation are shown before and during paralysis after $10 \mathrm{~min}$ treatment with tetramisole. Light grey indicates the exposure period of the worm to $\mathrm{NaCl}$ solution. Lightly colored shaded areas in (D) and (E) denote standard error. Average changes in fluorescence intensity during the $6 \mathrm{~s}$ window of stable state of stimulation before and after exposure to the paralytic agent are denoted in (F). Statistical significance is denoted with asterisks. * $P=$ $0.0132 ; * * P=0.0077$, ANOVA with Bonferroni correction. $\mathrm{n}=5-9$ per strain. Initial stimulation is shown in (D), while stimulation during paralysis is shown in (E). 

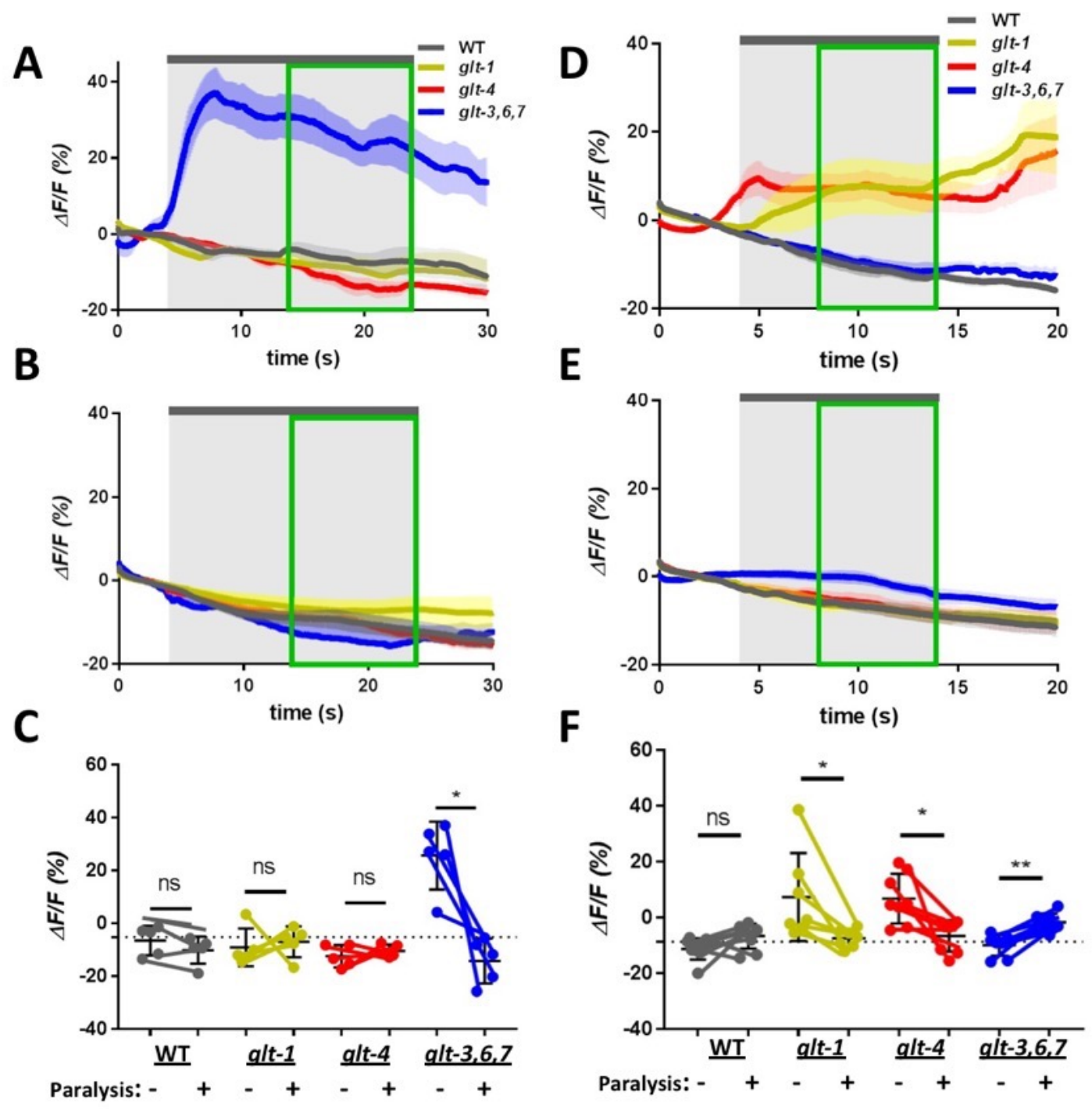

Figure 24. BDM-induced paralysis eliminates exaggerated responses in the ASH -> AVA synapse to 1 M glycerol. (A-C) Head muscle and pharyngeal paralysis induced by exposure to BDM caused loss in AVA response of glt-3,6,7 mutants to stimulation by $1 \mathrm{M}$ glycerol. Changes in AVA GCaMP intensity in response to 1 $\mathrm{M}$ glycerol stimulation are shown before and during paralysis after 10 min treatment with BDM. Light grey indicates the exposure period of the worm to glycerol solution. Lightly colored shaded areas in (A) and (B) denote standard error. Average changes in fluorescence intensity during the $10 \mathrm{~s}$ window of stable state of stimulation before and after exposure to the paralytic agent are denoted in (C). Statistical significance is denoted with asterisks. $* P=0.0106$, ANOVA with Bonferroni correction. $\mathrm{n}=5$ per strain. Initial stimulation is shown in (A), while stimulation during paralysis is shown in (B).

Putative spillover from ASEL onto AVA is eliminated by BDM-induced head paralysis. (D-F) Head muscle and pharyngeal paralysis induced by exposure to BDM caused loss in AVA response of glt- 1 and glt-4 mutants to stimulation by $1 \mathrm{mM} \mathrm{NaCl}$. Changes in AVA GCaMP intensity in response to $1 \mathrm{mM} \mathrm{NaCl}$ stimulation are shown before and during paralysis after 10 min treatment with BDM. Light grey indicates the exposure period of the worm to $\mathrm{NaCl}$ solution. Lightly colored shaded areas in (D) and (E) denote standard error. Average changes in fluorescence intensity during the $6 \mathrm{~s}$ window of stable state of stimulation before and after exposure to the paralytic agent are denoted in (F). Statistical significance is denoted with asterisks. ${ }^{*} P=0.0330$ for $g l t-1$ and 0.0145 for $g l t-4 ; * * P=0.0016$, ANOVA with Bonferroni correction. $\mathrm{n}=7-9$ per strain. Initial stimulation is shown in (D), while stimulation during paralysis is shown in (E). 

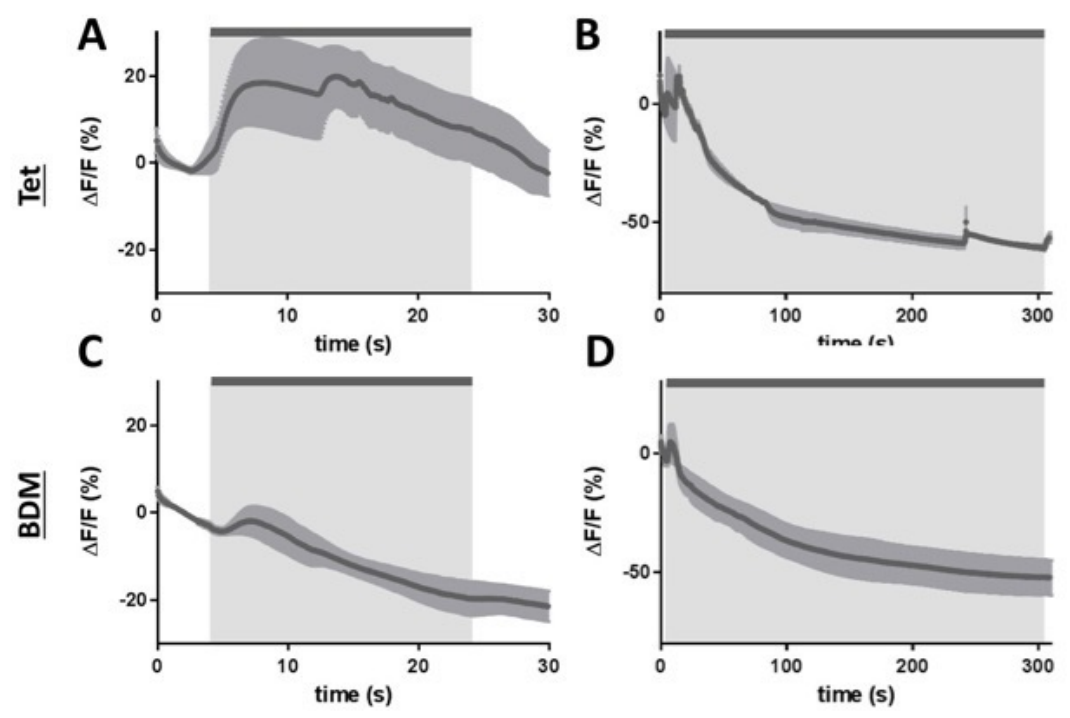

Figure 25. AVA displays response upon initial exposure to $2 \mathrm{mM}$ tetramisole or 0.3 M BDM.

WT animals expressing GCaMP in AVA neurons were imaged. $\mathbf{A}$ and $\mathbf{B}$ : exposure to Tetramisole; $\mathbf{C}$ and D: exposure to BDM. Light gray shading in $\mathbf{A}$ and $\mathbf{C}$ indicates initial $20 \mathrm{~s}$ period of exposure to the paralyzing agent, beginning at $t=3 \mathrm{~s}$, while $\mathbf{B}$ and $\mathbf{D}$ display a $5 \mathrm{~min}$ prolonged exposure. Light colored shading around each curve indicate SEM. $\mathrm{n}=2-5$.

\subsection{Discussion and Conclusion}

The spatial precision of neurotransmitter signaling in the nervous system is of paramount importance for synaptic physiology, dictating the precise alignment of transmitter release sites and postsynaptic receptors ${ }^{62}$. Our study focuses on synaptic clearance of Glu, a prevalent neurotransmitter, in a compact nervous system lacking spines and glia-mediated anatomical separation between synapses. We find that in the nematode nervous system, synaptic fidelity and circuit resolution is maintained by a robust system of proximal and distal Glu clearance. We observe that specific GluTs have a privileged role in preserving accuracy and preventing spillover in specific circuits. We affirm that the privileged role of distal GluTs ( $g l t-3, g l t-6, \&$ glt-7) on the ASH -> AVA/AVD/AVE avoidance circuit, seen previously at the behavioral level ${ }^{35}$, can be observed at the neurophysiological level as hyperactivation of somatic $\mathrm{Ca}^{2+}$ responses and dendritic Glu increase in postsynaptic AVA neurons (Figure 7) following Glu release from ASH (Figure 8). Distal GluTs expressed on the canal cell, through direct contact with pseudocoelomic body fluids, putatively maintain low ambient Glu concentrations to enable effective diffusion-mediated clearance. The concept of diffusion-mediated clearance in 
nematodes is in line with the anatomical observation that nematode synapses are aspiny and formed en passant ${ }^{32,44,46}$, promoting perisynaptic diffusion. In contrast to the functional association of ASH synapses with distal GluTs, the accuracy of ASE -> AIA/AIB/AIY synapses in the $\mathrm{NaCl}$ upstep sensing chemoattraction circuit is more influenced by the activity of the proximal GluTs, encoded by glt- 1 and $g l t-4$ (Figures 10, 11): in the absence of these proximal GluTs, ASE circuit resolution is lost, and Glu released from ASEL (Figure 14) spills over onto nearby circuits. The localization of ASE -> AIA/AIB/AIY synapses to the outer rim of the nerve ring (Figures $19 \& 20)$ offers a reasonably straightforward explanation to the privileged role of proximal GluTs on these synapses, as these synapses are closer to the glt-1 -expressing hypodermis, head muscles, and glia. While stimulation of ASEL with low salt upstep results in AVA excitation in proximal glt mutants, we were unable to induce the same effect with salt downstep stimulation of ASER. The reason for this discrepancy is currently unknown. Possible explanations include differences in the mode of Glu release, since ASEL is an ON-cell, which releases Glu only upon stimulation, whereas ASER is an OFF-cell, whose default is to release Glu constitutively ${ }^{40,43}$. Another potential factor could be that to create spillover from ASER to AVA one needs to eliminate both proximal and distal GluTs, which is a condition we could not test. This and other potential explanations might be related to a difference in the type or location of synapses used in upstep vs downstep signaling ${ }^{42}$, or on the effect of previous culturing conditions $^{63}$.

It is interesting to note that the proximal and distal GluTs are distinguished by significant differences in the sequence of the transporter's core domain, making nematode distal GluTs unique among all other SLC1A members. The ability of ectopic over-expression of noncanonical SLC1A GLT-3 to compensate for the absence of the canonical SLC1A GLT-1 
indicates that this non-canonical SLC1A transporter is still a bona fide GluT. And yet, the inability of the canonical SLC1A GLT-1 to compensate for the absence of the non-canonical SLC1A GLT-3 (even when overexpressed) indicates that the non-canonical SLC1A GLT-3 has unique functional features. These observations support that even a considerably divergent core domain of GluT can function in Glu transport, while also highlighting the significance of unexplored structural features in non-canonical GluTs. Recent studies of canonical members of the SLC1A family provide insights into the role of different core residues in the binding of substrates and the conformational changes that these transporters go through ${ }^{64-67}$. It will be interesting to evaluate how the modified core of the non-canonical SLC1A members, such as GLT-3, fit into these functional models.

The location of ASH -> AVA/AVD/AVE synapses closer to the inner rim of the nerve ring and their functional association with distal GluTs (maintaining ambient Glu concentrations) is particularly intriguing. Careful examination of anatomical data in WormAtlas brought an important notion to our attention, namely that the space between the inner rim of the nerve ring and the isthmus of the pharynx is filled with pseudocoelomic body fluids, a compartment linked to fluids in the rest if the body. We propose that together with the intense mechanical agitation in this area, these body fluids might provide perfusion of the inner rim of the nerve ring, which is contiguous with the interstitial fluids in the neuropil extracellular space. Indeed, we observe that inhibiting fluid agitation (using two different paralyzing agents) obstructs chemical stimulation of AVA (either directly from ASH or by spillover from ASEL, Figure 23), probably due to the saturating effect of spontaneous Glu accumulation prior to stimulation (Figure 25). This notion is further supported by our recent observation that under conditions that cause glt-3 $\mathrm{KO}$-induced excitotoxicity, the most severely affected neurons are those that face the pseudocoelomic fluid ${ }^{52}$. 
In our model for Glu clearance, Glu secreted closer to the outer rim of the nerve ring is preferentially cleared by GluTs expressed on the large structures that surround the nerve ring (hypodermis, head muscles, and glia), while Glu released closer to the inner rim of the nerve ring is initially cleared by circulating body fluids, with subsequent distal uptake into the canal cell by distal GluTs (Figure 25). This clearance mechanism may also be applicable to a number of other neurotransmitters in C. elegans, which have been suggested to spill out extra-synaptically ${ }^{68-70}$.

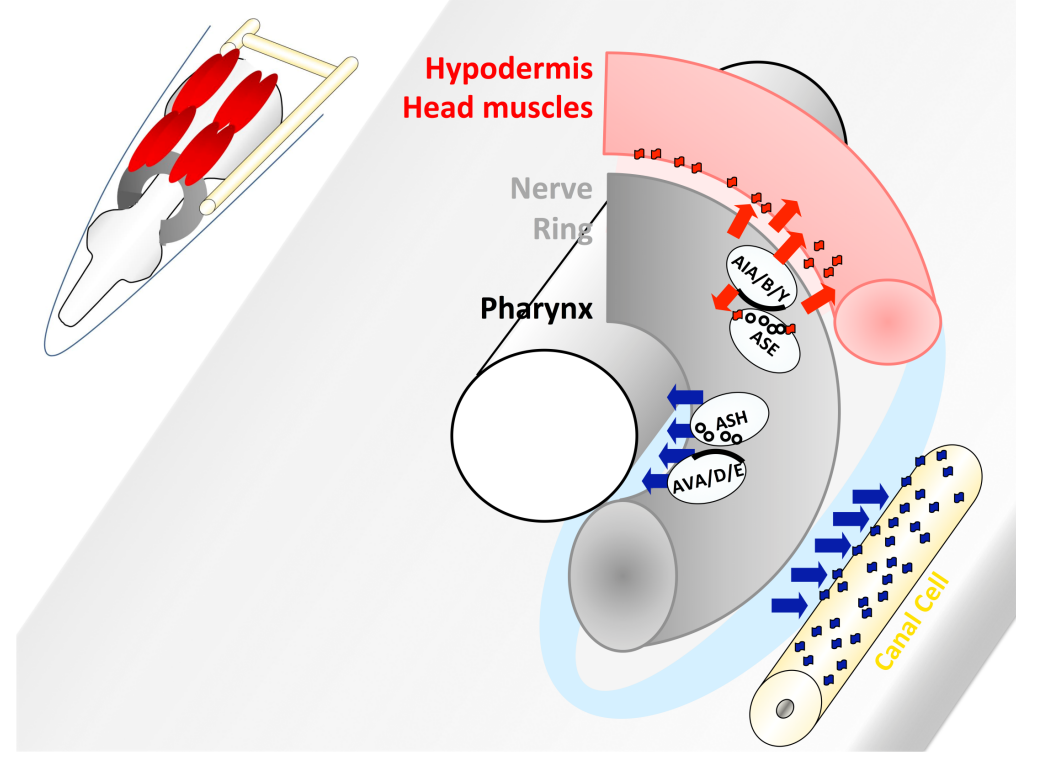

Figure 26. The two-tier model of Glu clearance in C. elegans.

Glu released in synapses that are closer to the outer rim of the nerve ring (such as in the ASE$>$ AIA/AIB/AIY circuit) is preferentially cleared by proximal GluTs expressed in head muscles (red) and hypodermis (not shown). Glu released in synapses that are closer to the inner rim of the nerve ring (such as in the ASH -> AVA/AVD/AVE circuit) is preferentially cleared by diffusion to the pseudocoelomic area between the inner rim of the nerve ring and the pharynx. Glu concentration in the pseudocoelomic fluid is kept low by distal GluTs on the canal cell. Agitation by pharyngeal activity and head movement might help circulate the pseudocoelomic fluid and perfuse the inner face of the nerve ring with fresh pseudocoelomic fluid that is low Glu in concentrations.

The incomplete isolation of synapses throughout animal phyla suggests that in other animals, circuit resolution may be maintained not only by anatomical separation, but also by a balance between physical isolation and functional means of Glu clearance from both synaptic and perisynaptic / interstitial spaces. Neurotransmitter escape into the immediate vicinity of the synaptic cleft does not immediately translate into spillover and ectopic responses in nearby synapses. In this view, rapid short-range escape of neurotransmitter out of the synapse is maintained as a viable clearance strategy due to the persistent (albeit slow) washout of the extrasynaptic space of the neuropil with interstitial fluid containing low ambient Glu 
concentrations (maintained distal GluTs). Vigorous clearance of Glu by perfusion can therefore compensate for anatomical shortcoming caused by the lack of glia isolation of synapses, and preserve circuit resolution. Our data supports the hypothesis that agitation of interstitial fluids and mechanical perfusion of the neuropil, together with subsequent clearance by distal GluTs, might be a considerable factor in Glu clearance in some key synapses in the nematode.

The structural characteristics of flat synapses ${ }^{71}$ seem to align with a clearance strategy that relies more heavily on clearance by diffusion and perfusion. It may be interesting to study the in vivo effect of perfusion in the nervous systems of higher animals, which possess a complete spectrum of synaptic morphologies, glial involvement ${ }^{71-73}$, and a dynamic range of levels of exposure to the glymphatic system ${ }^{2,74-76}$.

It is also worth noting that pulsations and vasomotion (which is slower and of higheramplitude) of mammalian brain arteries and paravascular CSF is readily observed ${ }^{77,78}$. In the murine brain, pulsatility or vasomotion of cerebral arteries has been recently shown to enhance interstitial fluid perfusion, and is suggested to augment clearance of extracellular solutes ${ }^{79-83}$. Pulsatility has been also used to preserve brain function after damage ${ }^{84,85}$. Furthermore, reduction of Glu clearance by agitation of interstitial fluid might have a pronounced role in brain damage during pulsation disturbances, and in conditions where extracellular space in the neuropil is reduced, such as brain edema ${ }^{86}$ and other pathological conditions ${ }^{87}$. Such disruptions may selectively affect glia-deprived flat synapses, where clearance by diffusion and bulk flow may be more critical. Continued study of the functional organization of neurotransmitter clearance in the nematode nervous system has the potential to elucidate unexpected and broadly applicable basic principles in the physiology of synaptic clearance. These insights might broaden the discussion 
of mechanisms that maintain specificity of synaptic signaling and the resolution of neuronal circuits. 


\subsection{Materials and Methods}

Strains and maintenance

All Caenorhabditis elegans strains were cultured at $20^{\circ} \mathrm{C}$ on MYOB plates ${ }^{88,89}$ with Escherichia coli strain OP50 as food source. Our wild type strain is Bristol N2. Other strains used for this study are: For behavioral analysis: VM1268: nmr-1(ak4) II; glr-2(ak10), glr-1(ky176) III ; ZB1113: glt-1(ok206) X ; ZB1096: glt-3(bz34) IV ; ZB1098: glt-4(bz69) X ; IMN16: glt-3(bz34), glt-6(tm1316), glt-7(tm1641) IV; For GCaMP imaging in AVA: QW625 (lin-15; zfIs42[P ${ }_{\text {rig- }}$ 3::GCaMP3::SL2::mCherry; lin-15(+)]); IMN18: glt-1(ok206) X; zfIs42 ; IMN19: glt-3(bz34), glt-6(tm1316), glt-7(tm1641) IV; zfIs42 ; IMN20: glt-4(bz69) X, zfIs42 ; For GCaMP imaging in

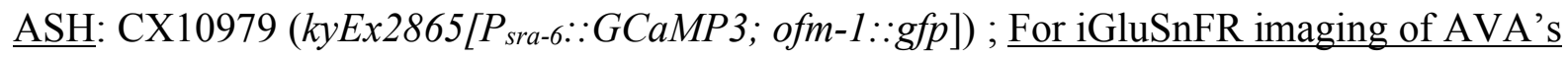
neurites: CX14652: kyEx4787 [P rig-3::iGluSnFR, unc-122::dsRed] ; IMN50: glt-1(ok206) X; kyEx4787; IMN51: glt-3(bz34), glt-6 (tm1316), glt-7 (tm1641) IV; kyEx4787. Details of the glt mutant strains were previously described ${ }^{35}$. The strains carrying glt mutations and AVA neurons expressing GCaMP or iGluSnFR (IMN18, IMN19, IMN20, IMN50 and IMN51) were generated by crosses between the corresponding glt mutants and the QW625 or CX14652 strain. QW625 $5^{30}$ was a gift from the Alkema lab (U. Mass. Med. Sch.) and was obtained via the Biron lab (U. Chicago $)^{90}$. The CX10979 55 and CX14652 ${ }^{5}$ strains were gifts from the Bargmann lab (Rockefeller U.). While we were able to generate animals that were triple KO mutants for all three distal GluTs ( $g l t-3$, glt-6, and glt-7) via mating, all attempts to generate double KO mutant strains for proximal GluTs glt-1 and glt-4 have been unsuccessful. For confirming that the signals seen in our AVA GCaMP imaging of glt strains arise in response to glutamatergic signals, we crossed our GCaMP-expressing glt mutants with eat-4 loss-of-function mutant MT6308 (eat-4 (ky5) III) (strains IMN52, IMN53, IMN54, and IMN55) For ASH-specific eat-4 
rescue in an eat-4 mutant background, glt mutant strains containing eat-4 loss-of-function mutation $(k y 55)$ were crossed with the TQ2905 (xuEx929: [P sra-6::eat-4(cDNA)::SL2::yfp1; $P_{n p r}$ -

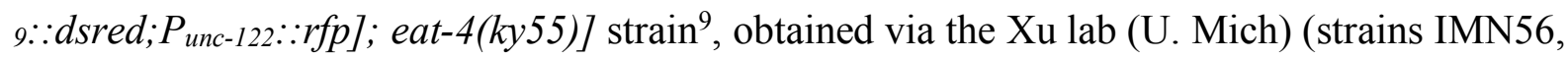
IMN57, IMN58, and IMN59). For ASEL-specific eat-4 rescue in an eat-4 mutant background, glt strains containing eat-4 loss-of-function mutation were injected with expression plasmids

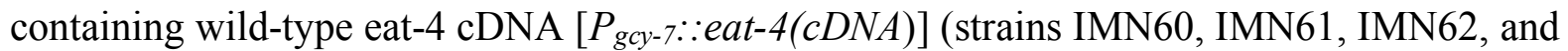
IMN63). For the promoter swap experiments, animals containing deletion mutations for either glt-1 or glt-3 were injected with one of the following constructs: $P_{g l t-3}:: g l t-$

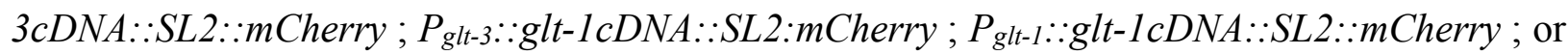
$P_{g l t-1}:: g l t-1 c D N A:: S L 2:: m$ Cherry (to generate strains IMN64, IMN65, IMN66, and IMN67).

\section{Molecular biology}

For all plasmid constructs with cDNA, total RNA was extracted from C. elegans Bristol strain N2 in TRIzol (Invitrogen) using RNeasy Mini Kit (Qiagen). RNA was used as template to create an cDNA prep using Invitrogen SuperScript ${ }^{\circledR}$ III and Oligo(dT)20 following manufacture’s protocol. All PCR fragments were amplified using NEB Q5 polymerase and were either purified via spin column purification or gel purified using NEB Monarch® DNA Gel Extraction Kit. Plasmids containing the SL2 trans-splicing signal results in a single transcript that is processed into two mRNAs and therefore two separate proteins. All plasmids were assembled via Gibson/HIFI assembly ${ }^{91}$ using NEB HiFi Assembly following manufacturer's protocol. Constructs were transformed in either Invitrogen DH5a Competent Cells (Cat \# 18265017) or Invitrogen One Shot ${ }^{\mathrm{TM}}$ competent cells (Cat\# C404010) 
Expression of EAT-4 in ASEL neuron:

The $1.3 \mathrm{~kb}$ of the 5' upstream regulatory region of the $g c y-7$ gene was PCR amplified from Bristol N2 genomic DNA ${ }^{92,93}$, using primers 5'-

AATGAAATTCGCCCACGGTTTTTTTTACCT and 5'-

CATGACGACATGATTATTTTCTTATGCTAAACTGGCAGAC. Total RNA was extracted

from C. elegans Bristol strain N2 and converted to cDNA. The cDNA prep was used as template to PCR amplify eat-4 cDNA using primers 5'-

AGAAAATAATCATGTCGTCATGGAACGAGGC and 5'-

CGTTAGTTAGCTACCACTGCTGATAATGCGGATTT. Plasmid vector pPD95.75 was PCR linearized and GFP sequence was removed using primers 5-

CAGTGGTAGCTAACTAACGAGTAATATTTAAATTTTCAGCATCTCGC and 5'GTGGGCGAATTTCATTTCCAAGTTGTTAGCGTATCCA. pPD95.75 was a gift from Andrew Fire (Addgene plasmid \# 1494). A Gibson assembly was performed and transformed into DH5 $\alpha$ cells.

Construction of $P_{\text {glt-3 } 3:: g l t-1 c D N A:: S L 2:: m \text { Cherry }}$

The glt-3 promoter was PCR amplified from 263 bp upstream the glt-3 ATG start site (Mano et al., 2007) from genomic DNA using primers 5'-

cttgcacttataGCTTTCGAATCGTTGTTTAATTTTACTGG containing 12 bp overlapping the ttTi5605 right recombination arm of pCFJ150 vector plasmid at the 5' end and 5'gttctatgttatgttagtatcattcgaaacatacCTTGTCTGTGTTGT with $34 \mathrm{bp}$ at the 5' end to include a synthetic intron before the cDNA sequence downstream. Vector pCFJ150 - pDESTttTi5605[R4R3] was a gift from Erik Jorgensen (Addgene plasmid \# 19329) ${ }^{94}$. The glt-1b cDNA was amplified from cDNA prep using primers 5'- 
gaatgatactaacataacatagaacattttcagATGGTATCCTGG with 33 bp at the 5' end to include a synthetic intro upstream and 5'- tgagacagcTCAATGAGTGTGCTTCTCGTCATCC with $9 \mathrm{bp}$ overlapping the SL2 sequence. The SL2::mCherry::UNC-54 3'UTR was amplified from XW09 plasmid using primers 5' - ctcattgaGCTGTCTCATCCTACTTTCACCTAGT with 8 bp overlapping glt-1 cDNA and 5'ttagagaatgtcGAAACAGTTATGTTTGGTATATTGGGAATGTATTCTG with 11 bp overlapping UNC-119 promoter in the pCFJ150 plasmid vector. XW09 was a gift from Kang Shen (Addgene plasmid \# 65833) ${ }^{95}$. The pCFJ150 vector was amplified using 5'cataactgtttcGACATTCTCTAATGAAAAAATCTTTCAGTTGAAATTGAAAATGAG with 13 bp overlapping the UNC-54 3'UTR and 5'tcgaaagcTATAAGTGCAAGTAAGATCAGTGTTTGTTTCG with 8 bp overlapping the $g l t-3$ promoter. Following Gibson assembly, plasmid was transformed into Invitrogen One Shot competent cells.

Construction of $P_{g l t-3}: \because g l t-3 c D N A:: S L 2:: m$ Cherry

The $g l t-3$ cDNA was amplified from cDNA prep using primers 5'ATGGGCATGAAGAAGGATCT and 5'- TTAGACAATATGGGTGTCAGACTTG and inserted in TOPO vector by Invitrogen Zero Blunt ${ }^{\mathrm{TM}} \mathrm{TOPO}^{\mathrm{TM}}$ vector (Cat\# 450245). The glt-3 cDNA was re-amplified from the $g l t-3$ cDNA TOPO plasmid to add HIFI overlapping sequences using primers 5' - cgaatgatactaacataacatagaacatttcagATGGGCATGAAGAAGGATCTTCTT with $34 \mathrm{bp}$ at the 5' end to include a synthetic intron and 5'gatgagacagcTTAGACAATATGGGTGTCAGACTTGTTCAA with 11 bp overlapping with SL2

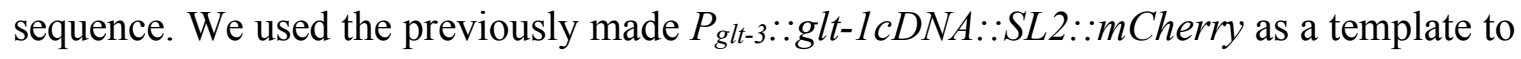

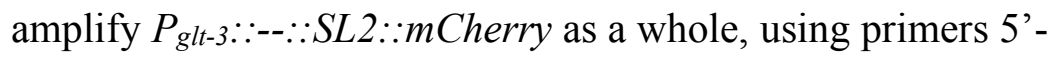


atattgtctaaGCTGTCTCATCCTACTTTCACCTAGT and 5'-

GTTCTATGTTATGTTAGTATCATTCGAAACATACCTTGTCTGTGTTGT. Following

Gibson assembly, plasmid was transformed into Invitrogen One Shot competent cells.

Construction of $P_{g l t-1: \because g l t-3 c D N A:: S L 2:: m \text { Cherry }}$

The $g l t-1$ promoter was PCR amplified from 4kb upstream of the ATG start codon ${ }^{33,35}$ from genomic DNA using primers 5'-GAAAATGCTCGAGCAGTTTTC and 5'-

CGTTTTGTGACTGTAGGTTTTGAAGG. Gel purified fragments were inserted into Invitrogen Zero Blunt $^{\mathrm{TM}}$ TOPO $^{\mathrm{TM}}$ vector (Cat\# 450245). The glt-1 promoter was re-amplified the from the glt-1 TOPO plasmid to include overlapping sequences using 5'-

cttgcacttaGAAAATGCTCGAGCAGTTTTCCAATTTTG with 10 bp overlapping the ttTi5605 right recombination sequence and 5'gttctatgttatgttagtatcattcgaaacataCGTTTTGTGACTGTAGGTTTTGAAGG with 34 bp to add a

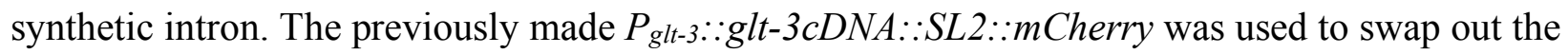
$P_{g l t-3}$ for $P_{g l t-1}$ using primers 5'- gagcattttcTAAGTGCAAGTAAGATCAGTGTTTGTTTCG and 5

CGAATGATACTAACATAACATAGAACATTTTCAGATGGGCATGAAGAAGGATCTTCT T. Following Gibson assembly, plasmid was transformed into Invitrogen One Shot competent cells.

Construction of $P_{g l t-1}: \because g l t-1 c D N A:: S L 2:: m$ Cherry

The previously made $P_{g l t-1}:: g l t-3 c D N A:: S L 2:: m$ Cherry plasmid was used to swap out the $g l t-3$ cDNA for glt-1 cDNA using Gibson assembly. The glt-1 cDNA was PCR amplified using the

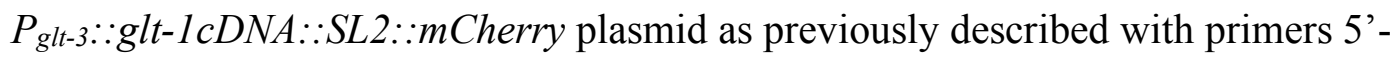
gaatgatactaacataacatagaacattttcagATGGTATCCTGG and 5'- 


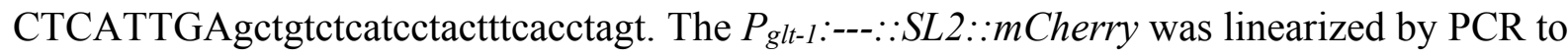
remove the $g l t-3$ cDNA sequence with primers 5'cttgcacttaGAAAATGCTCGAGCAGTTTTCCAATTTTG and 5'gttctatgttatgttagtatcattcgaaacataCGTTTTGTGACTGTAGGTTTTGAAGG, The fragments were circularize via Gibson assembly and transformed into Invitrogen One Shot competent cells.

Behavioral assays

Spontaneous mobility assay ${ }^{24,96,97}$ and nose touch assay ${ }^{98,99}$ were conducted as previously described. For the spontaneous mobility assay, the forward locomotion duration was determined until the individual worm either halts movement or reverses. For nose touch response we determined the number of worms responding (halting or reversing) or not responding (continuing forward mobility) to collision with an eyelash. For each session, about 30 animals of each genotype were tested.

Avoidance drop assay was conducted for testing worms' avoidance response to a range of $\mathrm{NaCl}$ concentrations as previously described ${ }^{8}$. We tested $\mathrm{NaCl}$ concentrations, ranging from $0.1 \mathrm{mM}$ to $1000 \mathrm{mM}$, dissolved in $1 \mathrm{mM} \mathrm{MgSO}_{4}, 1 \mathrm{mM} \mathrm{CaCl}_{2}$, and $5 \mathrm{mM} \mathrm{KPO}_{4}$. For each session, worms were transferred to an unseeded MYOB plate and left there for $15 \mathrm{~min}$ to remove remaining food on their body. Worms were then transferred to the assay plate and were given an additional 15 min to adjust to the buffer solution. A capillary tube was used to make a drop near the tail of a forward-moving worm. The drop immediately surrounds the whole worm body. A mere mechanosensory stimulation to the tail will stimulate the worm to rush forward, but chemorepellent properties of solutes sensed in the nose will cause the worm to halt or reverse. The worms' response within $4 \mathrm{~s}$ after the drop was denoted as 0 if the worms continued their 
forward movement, and as 1 if worms either stopped or reversed. To present the trend of avoidance over a range of $\mathrm{NaCl}$ concentrations, we used avoidance index equal to the percentage of worms avoiding specific $\mathrm{NaCl}$ concentration.

Microfluidics and imaging

Prior to imaging, adult worms were first transferred onto an unseeded MYOB holding plate. Worms were allowed to traverse the holding plate for 10 minutes, removing excess bacteria adhering to their bodies. For stimulation experiments with glycerol, S-basal buffer was added onto the holding plates for no more than 10 minutes before positioning of each individual worm into the worm channel of the microfluidics chip. For the salt stimulation experiments, a solution consisting of $1 \mathrm{mM} \mathrm{MgSO}_{4}, 1 \mathrm{mM} \mathrm{CaCl}_{2}$, and $5 \mathrm{mM} \mathrm{KPO}_{4}$ (salt stimulation buffer) was used instead $^{100}$.

The Chronis \& Bargmann worm behavioral chip (Purchased from MicroKosmos) was used for GCaMP and iGluSnFR imaging experiments ${ }^{6}$. This system allows for temporal stimulation of amphid sensory neurons located on the worm nose. Worms were physically restrained in a specially fitted channel (the worm trap) designed to hold adults without the use of paralytic agents that may interfere with normal physiology. A system of liquid streams controlled under laminar flow were manipulated to present either control buffer or stimulus to the worm nose ${ }^{6}$ via a three-way electric valve (Lee Company). GCaMP and iGluSnFR transients from live nematodes trapped in the chip were recorded as previously described by Chronis \& Bargmann. To generate the stimulant solutions, glycerol was dissolved in S Basal buffer to a final concentration of $1 \mathrm{M}^{6}$, while $1 \mathrm{mM} \mathrm{NaCl}$ was dissolved in salt stimulation buffer. For paralysis experiments, either $2 \mathrm{mM}$ tetramisole (Sigma) or 0.3 M BDM (2,3-Butanedione monoxime) (VWR) dissolved in either S-basal or salt stimulation buffer was introduced via the buffer 
channel of the microfluidics system to paralyze the anterior portion of the worm. Paralysis was induced by exposing the head of the immobilized animals contained in the worm trap of the microfluidics chamber to the paralytic agents for no more than 10 minutes.

Our imaging system consists of a Zeiss Axiovert 200 M motorized inverted microscope, Lumencor SOLA solid state white light source, Ludl filter wheel controller, Q Imaging EXiTM Blue camera, and ValveBank4 controller (AutoMate). Metamorph software (Molecular Devices) was used for image processing and acquisition. GCaMP transients were captured with a $63 \mathrm{x}$ objective lens (10 frames/s), while iGluSnFR transients were captured at 40x magnification (3 frames/s) for each experiment duration.

We used $\Delta \mathrm{F} / \mathrm{F}$ to indicate change in fluorescence intensity. $\mathrm{F}$ was defined as the baseline fluorescence intensity of AVA during a period of either 3 seconds (for recording effects of salt stimulation in AVA GCaMP and the iGluSnFR experiments) or 4 seconds (for recording AVA GCaMP signals during response to glycerol). The time windows for calculating intensity values for comparison of averages of relative stable states for each experiment was determined based on prior observations of the timing of neuronal responses, ensuring that the rise and relative stable period in signal intensity could be captured across all imaged strains. Before recording, worms were held for one minute in the microfluidics chamber in the presence of blue light to eliminate avoidance mediated through the photoreceptor protein LITE-1 ${ }^{101}$. Intensity measurements were restricted to AVA cell body for GCaMP imaging, and its proximal neuronal process for iGluSnFR imaging. This was achieved by first setting an inclusive intensity threshold to define the range of fluorescence to capture (i.e., neuronal soma or process, which has higher intensity compared to other cells and structures), then defining a region of interest (ROI) to capture from. To restrict iGluSnFR measurements to AVA neuronal processes in the absence of animal 
paralysis, the focus was adjusted so that the protruding ASH-AVA synapse ${ }^{7}$ is the only definable object in view, and can be reliably tracked within an ROI. Reporter transients were analyzed by comparing subsequent intensity readouts to the baseline intensity, expressed as a change in percentage. For both GCaMP and iGluSnFR imaging, intensity values of light objects were defined and quantified by Metamorph's Inclusive Threshold function within the ROI for each timepoint.

\section{Statistical analysis}

All statistical analyses was performed utilizing GraphPad Prism software. For the nose touch and the drop test assays, we used the Student's t-test with Welch's correction to compare significance of differences between mutant and N2 control strains. For GCaMP and iGluSnFR data, ANOVA with post hoc Bonferroni test was used for multiple group comparison of their means. Error bars denote SEM, and statistical significance is noted with * based on $\mathrm{P}$ values.

\subsection{Acknowledgements}

I would like to thank KyungWha Lee and Jenny Wong and for their instrumental roles in starting the GCaMP imaging and behavioral experiments for this project. I thank Irving Estevez for plasmid construction and microinjection of strains. I would also like to thank Sruti Patoori, Adanna Alexander, Zelda Mendelowitz, Ayesha Chowdhury, Ji-Sup Yang, and other members from both the Li and Emerson labs for the many insightful discussions and help with the molecular biology. I thank Behanyely Johnson for help with PCRs and gels. I would also like to express tremendous thanks to M. Katz, S. Shaham, C. Bargmann, and S. Chalasani for their 
extensive guidance in setting up and running the microfluidics system. I thank C. Bargmann, M. Alkema, and D. Biron for gift of C. elegans GCaMP strains, and D. Hall \& S. Emmons for guidance on worm connectivity. 


\subsection{References}

1. Vandenberg, R. J. \& Ryan, R. M. Mechanisms of Glutamate Transport. Physiol. Rev. 93, $1621-1657$ (2013).

2. Nicholson, C. \& Hrabětová, S. Brain Extracellular Space: The Final Frontier of Neuroscience. Biophys. J. 113, 2133-2142 (2017).

3. Mano, I., Straud, S. \& Driscoll, M. Caenorhabditis elegans glutamate transporters influence synaptic function and behavior at sites distant from the synapse. J. Biol. Chem. 282, 3441234419 (2007).

4. Katz, M., Corson, F., Iwanir, S., Biron, D. \& Shaham, S. Glia Modulate a Neuronal Circuit for Locomotion Suppression during Sleep in C. elegans. Cell Rep. 22, 2601-2614 (2018).

5. Marvin, J. S. et al. An optimized fluorescent probe for visualizing glutamate neurotransmission. Nat. Methods 10, 162-170 (2013).

6. Chronis, N., Zimmer, M. \& Bargmann, C. I. Microfluidics for in vivo imaging of neuronal and behavioral activity in Caenorhabditis elegans. Nat. Methods 4, 727-731 (2007).

7. Altun, Z. F. et al. Wormatlas. http://www.wormatlas.org/ (2002).

8. Chatzigeorgiou, M., Bang, S., Hwang, S. W. \& Schafer, W. R. tmc-1 encodes a sodiumsensitive channel required for salt chemosensation in C. elegans. Nature 494, 95-99 (2013).

9. Piggott, B. J., Liu, J., Feng, Z., Wescott, S. A. \& Xu, X. Z. S. The Neural Circuits and Synaptic Mechanisms Underlying Motor Initiation in C. elegans. Cell 147, 922-933 (2011).

10. de Bono, M. \& Villu Maricq, A. Neuronal Substrates of Complex Behaviors in C. elegans. Annu. Rev. Neurosci. 28, 451-501 (2005). 
11. Bastug, T. et al. Position of the Third $\mathrm{Na}+$ Site in the Aspartate Transporter Glt Ph and the Human Glutamate Transporter ,. PLoS ONE 7, 13-17 (2012).

12. Tzingounis, A. V. \& Wadiche, J. I. Glutamate transporters: Confining runaway excitation by shaping synaptic transmission. Nat. Rev. Neurosci. 8, 935-947 (2007).

13. Levy, S., Kafri, M., Carmi, M. \& Barkai, N. The Competitive Advantage of a DualTransporter System. Science 334, 1408-1412 (2011).

14. Bailey, C. J. Renal glucose reabsorption inhibitors to treat diabetes. Trends Pharmacol. Sci. 32, 63-71 (2011).

15. Closs, E. I., Albritton, L. M., Kim, J. W. \& Cunningham, J. W. Identification of a Low Affinity, High Capacity Transporter of Cationic Amino Acids in Mouse Liver. J. Biol. Chem. 286, 7538-7544 (1993).

16. Ryan, R. M., Mitrovic, A. D. \& Vandenberg, R. J. The Chloride Permeation Pathway of a Glutamate Transporter and Its Proximity to the Glutamate Translocation Pathway. J. Biol. Chem. 279, 20742-20751 (2004).

17. Ryan, R. M. \& Mindell, J. A. The uncoupled chloride conductance of a bacterial glutamate transporter homolog. Nat. Struct. Amp Mol. Biol. 14, 365 (2007).

18. Cheng, M. H., Torres-Salazar, D., Gonzalez-Suarez, A. D., Amara, S. G. \& Bahar, I. Substrate transport and anion permeation proceed through distinct pathways in glutamate transporters. eLife 6, (2017).

19. Zhang, Y. I-TASSER server for protein 3D structure prediction. BioMed Cent. Bioinforma. 9, 1-8 (2008).

20. Ambrish, R., Kucukural, A. \& Zhang, Y. I-TASSER: a unified platform for automated protein structure and function prediction. Nat. Protoc. 5, 725-738 (2010). 
21. Zhang, Y. The I-TASSER Suite: protein structure and function prediction. Nat. Methods 12, $1-8(2015)$.

22. Schrödinger, L. L. C. The PyMOL Molecular Graphics System. (2018).

23. Brockie, P. Ionotropic glutamate receptors: genetics, behavior and electrophysiology. WormBook (2006) doi:10.1895/wormbook.1.61.1.

24. Mellem, J. E., Brockie, P. J., Zheng, Y., Madsen, D. M. \& Maricq, A. V. Decoding of Polymodal Sensory Stimuli by Postsynaptic Glutamate Receptors in C. elegans. Neuron 36, 933-944 (2002).

25. Tian, L. et al. Imaging neural activity in worms, flies and mice with improved GCaMP calcium indicators. Nat. Methods 6, 875-881 (2009).

26. Zhao, Y. et al. An expanded palette of genetically encoded Ca2+ indicators. Science 557, 1888-1891 (2011).

27. Nakai, J., Ohkura, M. \& Imoto, K. A high signal-to-noise Ca2+ probe composed of a single green fluorescent protein. Nat. Biotechnol. 19, 137-141 (2001).

28. Chalasani, S. H. et al. Dissecting a circuit for olfactory behaviour in Caenorhabditis elegans. Nature 450, 63 (2007).

29. Chronis, N. Worm chips: Microtools for C. elegans biology. Lab Chip 10, $432-437$ (2010).

30. Shipley, F. B., Clark, C. M., Alkema, M. J. \& Leifer, A. M. Simultaneous optogenetic manipulation and calcium imaging in freely moving C. elegans. Front. Neural Circuits $\mathbf{8}$, (2014).

31. Cho, J. Y. \& Sternberg, P. W. Multilevel Modulation of a Sensory Motor Circuit during C. elegans Sleep and Arousal. Cell 156, 249-260 (2014). 
32. Cook, S. J. et al. Whole-animal connectomes of both Caenorhabditis elegans sexes. Nature 571, 63-71 (2019).

33. Katz, M. et al. Glutamate spillover in C. elegans triggers repetitive behavior through presynaptic activation of MGL-2/mGluR5. (2019) doi:10.1101/415828.

34. Lee, R. Y. N., Sawin, E. R., Chalfie, M., Horvitz, H. R. \& Avery, L. EAT-4, a Homolog of a Mammalian Sodium-Dependent Inorganic Phosphate Cotransporter, Is Necessary for Glutamatergic Neurotransmission in Caenorhabditis elegans. J. Neurosci. 19, 159-167 (1999).

35. Mano, I., Straud, S. \& Driscoll, M. Caenorhabditis elegans Glutamate Transporters Influence Synaptic Function and Behavior at Sites Distant from the Synapse. J. Biol. Chem. 282, 34412-34419 (2007).

36. Gibson, C. L. et al. Glial loss of the metallo $\beta$-lactamase domain containing protein, SWIP10, induces age- and glutamate-signaling dependent, dopamine neuron degeneration. PLOS Genet. 14, e1007269 (2018).

37. Serrano-Saiz, E. et al. SLC17A6/7/8 Vesicular Glutamate Transporter Homologs in Nematodes. Genetics 214, 163-178 (2020).

38. Miyaji, T., Omote, H. \& Moriyama, Y. Functional characterization of vesicular excitatory amino acid transport by human sialin: Functional characterization of human sialin mutants. J. Neurochem. 119, 1-5 (2011).

39. Eriksen, J., Li, F. \& Edwards, R. H. The mechanism and regulation of vesicular glutamate transport: Coordination with the synaptic vesicle cycle. Biochim. Biophys. Acta BBA Biomembr. 1862, 183259 (2020). 
40. Suzuki, H. et al. Functional asymmetry in Caenorhabditis elegans taste neurons and its computational role in chemotaxis. Nature 454, 114-117 (2008).

41. Wang, L. et al. A Gustatory Neural Circuit of Caenorhabditis elegans Generates MemoryDependent Behaviors in $\mathrm{Na}^{+}$Chemotaxis. J. Neurosci. 37, 2097-2111 (2017).

42. Kuramochi, M. \& Doi, M. An Excitatory/Inhibitory Switch From Asymmetric Sensory Neurons Defines Postsynaptic Tuning for a Rapid Response to $\mathrm{NaCl}$ in Caenorhabditis elegans. Front. Mol. Neurosci. 11, (2019).

43. Leinwand, S. G. \& Chalasani, S. H. Neuropeptide signaling remodels chemosensory circuit composition in Caenorhabditis elegans. Nat. Neurosci. 16, 1461-1467 (2013).

44. White, J. G., Southgate, E., Thomson, J. N. \& Brenner, S. The Structure of the Nervous System of the Nematode Caenorhabditis elegans. Philos. Trans. R. Soc. Lond. 314, 1-340 (1986).

45. Jarrell, T. A. et al. The Connectome of a Decision-Making Neural Network. Science 337, $437-444$ (2012).

46. Brittin, C. A., Cook, S. J., Hall, D. H., Emmons, S. W. \& Cohen, N. A multi-scale brain map derived from whole-brain volumetric reconstructions. Nature 591, 105-110 (2021).

47. Kullmann, D. M. \& Asztely, F. Extrasynaptic glutamate spillover in the hippocampus: evidence and implications. Trends Neurosci. 21, 8-14 (1998).

48. Bergles, D. E., Diamond, J. S. \& Jahr, C. E. Clearance of glutamate inside the synapse and beyond. Curr. Opin. Neurobiol. 9, 293-298 (1999).

49. Diamond, J. S. A broad view of glutamate spillover. Nat. Neurosci. 5, 291-292 (2002).

50. Kandel, E. R., Schwartz, J. H., Jessell, T. M., Siegelbaum, S. A. \& Hudspeth, A. J. Principles of Neural Science 5th Edition. (McGraw Hill, 2013). 
51. Mano, I. \& Driscoll, M. Caenorhabditis elegans glutamate transporter deletion induces AMPA-receptor/adenylyl cyclase 9-dependent excitotoxicity. J. Neurochem. 108, 13731384 (2009).

52. Feldmann, K. G. et al. Non-Canonical Activation of CREB Mediates Neuroprotection in a C. elegans Model of Excitotoxic Necrosis. (2019) doi:10.1101/261420.

53. Lewis, J. A., Fleming, J. T., McLafferty, S., Murphy, H. \& Wu, C. The Levamisole Receptor, a Cholinergic Receptor of the Nematode Caenorhabditis elegans. Mol. Pharmacol. 31, 185-193 (1986).

54. Hendricks, M., Ha, H., Maffey, N. \& Zhang, Y. Compartmentalized calcium dynamics in a C. elegans interneuron encode head movement. Nature 487, 99-103 (2012).

55. Larsch, J., Ventimiglia, D., Bargmann, C. I. \& Albrecht, D. R. High-throughput imaging of neuronal activity in Caenorhabditis elegans. Proc. Natl. Acad. Sci. 110, E4266-E4273 (2013).

56. Kato, S., Xu, Y., Cho, C. E., Abbott, L. F. \& Bargmann, C. I. Temporal Responses of $C$. elegans Chemosensory Neurons Are Preserved in Behavioral Dynamics. Neuron 81, 616$628(2014)$

57. Schrödel, T., Prevedel, R., Aumayr, K., Zimmer, M. \& Vaziri, A. Brain-wide 3D imaging of neuronal activity in Caenorhabditis elegans with sculpted light. Nat. Methods 10, 10131020 (2013).

58. Feng, Z. et al. A C. elegans Model of Nicotine-Dependent Behavior: Regulation by TRPFamily Channels. Cell 127, 621-633 (2006). 
59. Sherlekar, A. L. et al. The C. elegans Male Exercises Directional Control during Mating through Cholinergic Regulation of Sex-Shared Command Interneurons. PLoS ONE 8, e60597 (2013)

60. Gordus, A., Pokala, N., Levy, S., Flavell, S. W. \& Bargmann, C. I. Feedback from Network States Generates Variability in a Probabilistic Olfactory Circuit. Cell 161, 215-227 (2015).

61. Goodman, M. B. \& Chalfie, M. Paralysis of worms by 2,3-butanedione monoxime (BDM). Worm Breed. Gaz. 15, 9 (1998).

62. Biederer, T., Kaeser, P. S. \& Blanpied, T. A. Transcellular Nanoalignment of Synaptic Function. Neuron 96, 680-696 (2017).

63. Kunitomo, H. et al. Concentration memory-dependent synaptic plasticity of a taste circuit regulates salt concentration chemotaxis in Caenorhabditis elegans. Nat. Commun. 4, (2013).

64. Wang, J., Zhang, K., Goyal, P. \& Grewer, C. Mechanism and potential sites of potassium interaction with glutamate transporters. J. Gen. Physiol. 152, e202012577 (2020).

65. Alleva, C., Machtens, J.-P., Kortzak, D., Weyand, I. \& Fahlke, C. Molecular Basis of Coupled Transport and Anion Conduction in Excitatory Amino Acid Transporters. Neurochem. Res. (2021) doi:10.1007/s11064-021-03252-x.

66. Chen, I. et al. Glutamate transporters have a chloride channel with two hydrophobic gates. Nature 591, 327-331 (2021).

67. Qiu, B., Matthies, D., Fortea, E., Yu, Z. \& Boudker, O. Cryo-EM structures of excitatory amino acid transporter 3 visualize coupled substrate, sodium, and proton binding and transport. Sci. Adv. 7, eabf5814 (2021). 
68. Chase, D. L., Pepper, J. S. \& Koelle, M. R. Mechanism of extrasynaptic dopamine signaling in Caenorhabditis elegans. Nat. Neurosci. 7, 1096-1103 (2004).

69. Jafari, G., Xie, Y., Kullyev, A., Liang, B. \& Sze, J. Y. Regulation of Extrasynaptic 5-HT by Serotonin Reuptake Transporter Function in 5-HT-Absorbing Neurons Underscores Adaptation Behavior in Caenorhabditis elegans. J. Neurosci. 31, 8948-8957 (2011).

70. Jobson, M. A. et al. Spillover Transmission Is Mediated by the Excitatory GABA Receptor LGC-35 in C. elegans. J. Neurosci. 35, 2803-2816 (2015).

71. Segal, M. Dendritic spines, synaptic plasticity and neuronal survival: activity shapes dendritic spines to enhance neuronal viability: Dendritic spine plasticity. Eur. J. Neurosci. 31, 2178-2184 (2010).

72. Harris, K. M. Structure, development, and plasticity of dendritic spines. Curr. Opin. Neurobiol. 9, 343-348 (1999).

73. Thomas, C. G., Tian, H. \& Diamond, J. S. The Relative Roles of Diffusion and Uptake in Clearing Synaptically Released Glutamate Change during Early Postnatal Development. J. Neurosci. 31, 4743-4754 (2011).

74. Da Mesquita, S., Fu, Z. \& Kipnis, J. The Meningeal Lymphatic System: A New Player in Neurophysiology. Neuron 100, 375-388 (2018).

75. Rasmussen, M. K., Mestre, H. \& Nedergaard, M. The glymphatic pathway in neurological disorders. Lancet Neurol. 17, 1016-1024 (2018).

76. Henneberger, C. et al. LTP Induction Boosts Glutamate Spillover by Driving Withdrawal of Perisynaptic Astroglia. Neuron 108, 919-936.e11 (2020). 
77. Hadaczek, P. et al. The "Perivascular Pump" Driven by Arterial Pulsation Is a Powerful Mechanism for the Distribution of Therapeutic Molecules within the Brain. Mol. Ther. 14, 69-78 (2006).

78. Wagshul, M. E., Eide, P. K. \& Madsen, J. R. The pulsating brain: A review of experimental and clinical studies of intracranial pulsatility. Fluids Barriers CNS 8, 5 (2011).

79. Iliff, J. J. et al. Cerebral Arterial Pulsation Drives Paravascular CSF-Interstitial Fluid Exchange in the Murine Brain. J. Neurosci. 33, 18190-18199 (2013).

80. Mestre, H. et al. Flow of cerebrospinal fluid is driven by arterial pulsations and is reduced in hypertension. Nat. Commun. 9, 4878 (2018).

81. Nedergaard, M. \& Goldman, S. A. Glymphatic failure as a final common pathway to dementia. Science 370, 50-56 (2020).

82. van Veluw, S. J. et al. Vasomotion as a Driving Force for Paravascular Clearance in the Awake Mouse Brain. Neuron 105, 549-561.e5 (2020).

83. Wardlaw, J. M. et al. Perivascular spaces in the brain: anatomy, physiology and pathology. Nat. Rev. Neurol. 16, 137-153 (2020).

84. Cohn, W. E., Timms, D. L. \& Frazier, O. H. Total artificial hearts: past, present, and future. Nat. Rev. Cardiol. 12, 609-617 (2015).

85. Vrselja, Z. et al. Restoration of brain circulation and cellular functions hours post-mortem. Nature 568, 336-343 (2019).

86. Sherpa, A. D., van de Nes, P., Xiao, F., Weedon, J. \& Hrabetova, S. Gliotoxin-induced swelling of astrocytes hinders diffusion in brain extracellular space via formation of deadspace microdomains: Astrocytic Swelling Hinders Extracellular Diffusion. Glia 62, 10531065 (2014). 
87. Arbel-Ornath, M. et al. Interstitial fluid drainage is impaired in ischemic stroke and Alzheimer's disease mouse models. Acta Neuropathol. (Berl.) 126, 353-364 (2013).

88. Brenner, S. The Genetics of Caenorhabditis elegans. Genetics 77, 71-94 (1974).

89. Church, D. L., Guan, K.-L. \& Lambie, E. J. Three genes of the MAP kinase cascade, mek-2, mpk-1/sur-1 and let-60 ras, are required for meiotic cell cycle progression in Caenorhabditis elegans. 11 (1995).

90. Iwanir, S. et al. The Microarchitecture of C. elegans Behavior during Lethargus:

Homeostatic Bout Dynamics, a Typical Body Posture, and Regulation by a Central Neuron. Sleep 36, 385-395 (2013).

91. Gibson, D. G. et al. Enzymatic assembly of DNA molecules up to several hundred kilobases. Nat. Methods 6, 343-345 (2009).

92. Yu, S., Avery, L., Baude, E. \& Garbers, D. L. Guanylyl cyclase expression in specific sensory neurons: A new family of chemosensory receptors. Proc. Natl. Acad. Sci. 94, 3384-3387 (1997).

93. Chang, S. A transcriptional regulatory cascade that controls left/right asymmetry in chemosensory neurons of C. elegans. Genes Dev. 17, 2123-2137 (2003).

94. Frøkjær-Jensen, C. et al. Single-copy insertion of transgenes in Caenorhabditis elegans. Nat. Genet. 40, 1375-1383 (2008).

95. Wei, X., Potter, C. J., Luo, L. \& Shen, K. Controlling gene expression with the Q repressible binary expression system in Caenorhabditis elegans. Nat. Methods 9, 391-395 (2012).

96. Zheng, Y., Brockie, P. J., Mellem, J. E., Madsen, D. M. \& Maricq, A. V. Neuronal Control of Locomotion in C. elegans Is Modified by a Dominant Mutation in the GLR-1 Ionotropic Glutamate Receptor. Neuron 24, 347-361 (1999). 
97. Brockie, P. J., Madsen, D. M., Zheng, Y., Mellem, J. \& Maricq, A. V. Differential Expression of Glutamate Receptor Subunits in the Nervous System of Caenorhabditis elegans and Their Regulation by the Homeodomain Protein UNC-42. J. Neurosci. 21, $1510-1522(2001)$

98. Kaplan, J. M. \& Horvitz, H. R. A dual mechanosensory and chemosensory neuron in Caenorhabditis elegans. Proc. Natl. Acad. Sci. 90, 2227-2231 (1993).

99. Hart, A. C., Sims, S. \& Kaplan, J. M. Synaptic code for sensory modalities revealed by $C$. elegans GLR-1 glutamate receptor. Nature 378, 82-85 (1995).

100. Oda, S., Tomioka, M. \& Iino, Y. Neuronal plasticity regulated by the insulin-like signaling pathway underlies salt chemotaxis learning in Caenorhabditis elegans. J. Neurophysiol. 106, 301-308 (2011).

101. Yemini, E. et al. NeuroPAL: A Multicolor Atlas for Whole-Brain Neuronal Identification in C. elegans. Cell 184, 272-288.e11 (2021). 


\section{Chapter 3}

Synaptic maintenance and differentiation of signals within a neuron where Glu is both an excitatory and inhibitory signal 


\subsection{Abstract}

In the mammalian CNS, Glutamate (Glu) plays major roles as an excitatory neurotransmitter, and Glu receptor subunits are well-characterized and abundant in the mammalian brain ${ }^{1}$. However, Glu is not solely excitatory in nature; it serves inhibitory roles in coupled conductance with chloride ions through the human retinal excitatory amino acid transporter, EAAT5, to negatively regulate constitutive release of excitatory Glu signals at retinal pre-synapses ${ }^{2,3}$. Building on our previous C. elegans model studying the roles of perfusion and bulk flow in facilitating Glu clearance to differentially localized GluTs, we explore how Glu signal fidelity is maintained within compartmentalized neuronal processes of a neuron that displays distinct and localized expression of excitatory and inhibitory Glu receptors. As opposed to exploring effective clearance and Glu spillover between neurons belonging to adjacent behavioral circuits, we explore the roles of perfusion and bulk flow in intraneuronal clearance, and how the same neurotransmitter can be deciphered to elicit either excitatory or inhibitory responses. By stimulating the symmetrical yet functionally distinct chemosensory ASE neurons, we quantify excitation and inhibition of postsynaptic somatic and neurite GCaMP response in interneuron AIB. 


\subsection{Introduction}

\subsubsection{Glu signaling in the ASE->AIB system salt chemotaxis circuit is asymmetrical and}

highly complex.

In C. elegans, the ASE neurons ASEL and ASER are a pair of left-right homologs that play a dominant role in salt chemotaxis ${ }^{4}$. Despite their homology, they display marked differences in gene expression, neuropeptide production, and ion preference ${ }^{5-8}$ in additional to functional asymmetry: while ASEL is an "ON-cell", stimulated by increases (upsteps) in $\mathrm{NaCl}$ concentration, ASER is an "OFF-cell", and is stimulated by decreases (downsteps) in $\mathrm{NaCl}$ concentration $^{4}$. This asymmetry also extends to behavioral output: ASEL promotes forward locomotion (runs), whereas ASER promotes direction changes (turns) ${ }^{4}$. The AIB neurons are downstream of and directly synapses with the ASE neurons, and is of particular interest for examination because it has been an intensively researched neuron for not only chemotaxis and chemosensation behaviors, but also locomotion in C. elegans ${ }^{9-11}$. While other neurons constitute the chemosensory / salt chemotaxis circuit (including AIA and AIY) downstream of ASE ${ }^{9,12}$, ablation of AIB interneurons results in drastically lengthened forward duration movements (averaging greater than 3 minutes, as opposed to short-duration forward movements of less than $20 \mathrm{~s}$ characteristic of the average wild-type animal) ${ }^{9}$. Along with AVA, AIB is also considered a "rich club neuron", one of six neurons that are rich in synapses and are involved in sensory information processing ${ }^{13,14}$. AIB interneurons are also precisely positioned to receive inputs from amphid sensory neurons and transduce outputs to motor neurons ${ }^{14}$. This architecture is consistent with AIB's role in processing amphid sensory stimuli to mediate locomotory strategies and serves as a key line of communication between two different strata in the nematode nerve ring ${ }^{14}$. 
Intriguingly, functional asymmetry has also recently been found to exist between the pair of AIB neurons; Rabinowitch et al. (2021) reported that AIBL GCaMP responses to excitatory stimulation by downstep in $\mathrm{NaCl}$ levels are more intense compared to responses in $\mathrm{AIBR}^{15}$. Previous literature and research on AIB had assumed AIBL/R functional symmetry, and as such did not disaggregate their data when collecting AIB responses to changes in $\mathrm{NaCl}$ levels. While the causes of this asymmetry have not been verified, recent connectome data from Witvliet et al. (2020), have uncovered differences in the number of chemical synapses formed between ASEL and ASER with AIBL and AIBR (Figure 27A). While the number of synapses with a presynaptic partner does not correlate with the strength of synaptic inputs received by the postsynaptic neurons ${ }^{16}$, this asymmetry in connectivity may be a contributing factor to differences in activity and post-synaptic responses observed between AIBL and AIBR ${ }^{15}$. Figure 27A demonstrates this asymmetry, illustrating that ASEL forms 7 chemical synapses with AIBL and 4 synapses with AIBR, while ASER forms 2 chemical synapses with AIBL and 10 synapses with AIBR. 


\section{A}

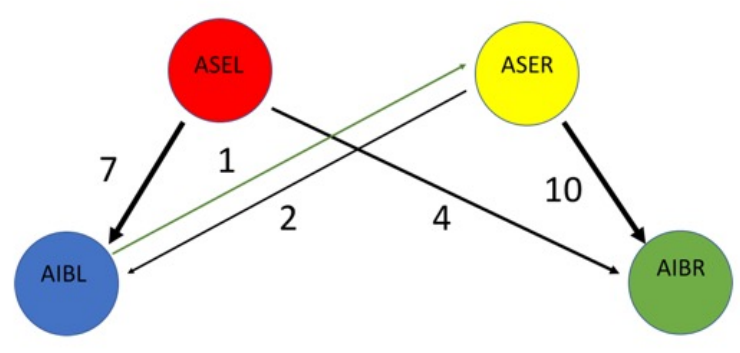

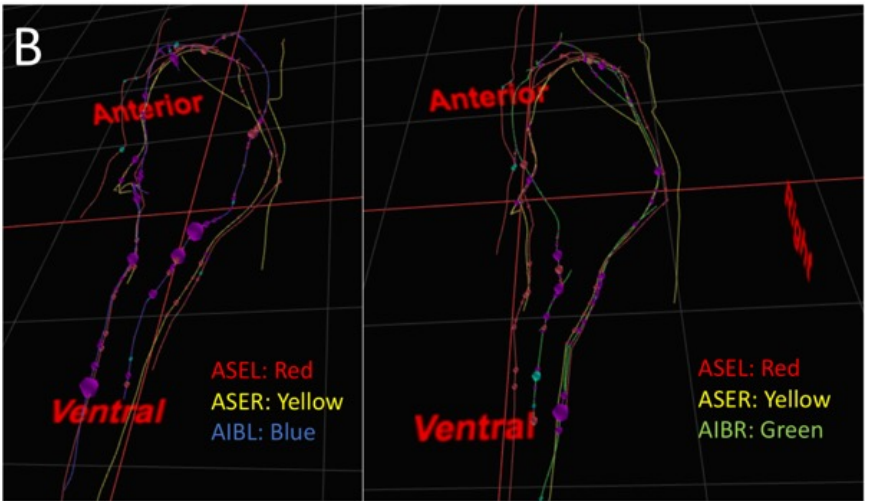

Figure 27. Visualization of ASE and AIB synaptic connections.

A. The number of ASEL and ASER synaptic connections can differ between the pair of AIB neurons. Diagram adapted from the EM data compiled by Witvliet et al. (2020) available on the Nemanode online resource (https://nemanode.org/). Black arrows indicate chemical synapses, while green arrow indicates unclassified synaptic connections. Numbers next to each arrow indicate the number of connections between the neurons connected by the arrow.

B. 3D reconstruction of neurons suggest that post-synaptic ASEL and distal AIB neurites do not appear to co-localize as strongly as depicted in previous studies. $3 \mathrm{D}$ volumetric reconstruction of ASEL (red), ASER (yellow), AIBL (blue), and AIBR (green) neurons, including neurites. Created using the Wormwiring online resource (https://wormwiring.org/apps/neuronMaps/).

\subsubsection{Location of ASER->AIB and ASEL->AIB and differential localization of GluRs may}

be a determinant of how Glu signals are interpreted by AIB.

While both ASE neurons form Glu synapses with interneuron AIB, their stimulation can have extremely disparate effects on the physiological neuronal activity of AIB. This difference has been shown to be dependent on which ASE neuron is the source of the Glu signal; AIB interneurons are activated by the downstep in $\mathrm{NaCl}$ concentration (by Glu released upon synchronous activation of the ASER neuron) ${ }^{11}$, while an upstep in $\mathrm{NaCl}$ concentration (resulting in activation of ASEL) has an inhibitory effect on $\mathrm{AIB}^{10}$. Kuramochi and Doi (2019) performed calcium imaging of AIB physiological activity in nematodes in response to changes in $\mathrm{NaCl}$ levels and explored how Glu can act as an excitatory or inhibitory signals on AIB, depending on whether the origin of the transmission is ASER or ASEL, respectively ${ }^{17}$. Their study had attributed the ability to distinguish between these Glu signals to two main factors at the AIB 
neurite: 1. Location of ASEL->AIB and ASER->AIB synapses and 2. Identity and localization patterns of GluRs with different properties. ASER synapses onto the proximal neurite region of AIB, which contains a higher concentration of ionotropic GluR GLR-1, and upon ASER stimulation contributes to the excitatory AIB response to $\mathrm{NaCl}$ downstep ${ }^{17,18}$. In contrast, ASEL synapses onto the distal neurite region of AIB, which has chloride-conducting GluCl GLC-3 clustered in puncta, and upon ASEL stimulation contributes to the inhibitory response to $\mathrm{NaCl}$ upstep $^{17,19}$. Taken together, spatial separation and colocalization of ASER and ASEL synapses with specific GluRs appear to be the primary decoders of Glu signaling within the AIB neurons. While Kuramochi and Doi's (2019) protein-reporter fusion experiments imply that the ASER and ASEL synapses neatly co-localize to separate regions of the AIB neurite ${ }^{17}, 3 \mathrm{D}$ volumetric reconstruction of these neurons tell a different story. We used the Wormwiring Neuron Maps app (https://wormwiring.org/apps/neuronMaps/) to specifically visualize the neurites and synapses of the pairs of ASE and AIB neurons ${ }^{20}$. A view of these spatial relations is depicted in Figure 27B: while the neurites of both ASEL and ASER make contact with the proximal neurite region of AIBL and AIBR (which has stronger localization of GLR-1 that contributes to the excitation response), there is a significant distance between the AIB distal neurites (which contains puncta of GLC-3 that contributes to the inhibition response). Given this distance, it is unclear whether efficiency and fidelity of inhibitory Glu neurotransmission is possible in Kuramochi and Doi's (2019) proposed model of AIB inhibition and excitation ${ }^{17}$. This raises the question of whether perfusion of Glu from ASE neurons, facilitated by nematode head movements and pharyngeal pumping, is able to gap the distance from the ASEL post-synapse to distal AIB neurites, just as our previous studies have found mechanical agitation of interstitial 
fluids to play a critical role in clearing Glu from AVA synapses on the inner nerve ring to distal GluTs expressed on canal cells ${ }^{21}$.

\subsection{Results}

\subsubsection{GluT function is needed to mediate effective clearance of ASE->AIB synapses.}

My previous experiments have indicated that perfusion is needed for effective clearance of AVA synapses located on the inner nerve ring of the nematode. While I had recorded synaptic spillover from the ASE circuit onto AVA, I had not directly measured Glu signaling or physiological activity of AIB. I wanted to explore if this phenomenon also presides over synaptic clearance of AIB. Based on the proximity of GLT-1 and GLT-4 -expressing structures to the ASE circuit on the outer rim of the nerve ring, we had predicted AIB to rely more heavily on proximal GluTs with a lesser dependence on perfusion in mediating effective clearance.

I imaged GCaMP6 strains expressing GCaMP6 specifically in AIB, capturing $\mathrm{Ca}^{2+}$ responses to changes (upstep and downstep) in low $\mathrm{NaCl}$ concentrations. I first began stimulating with a $50 \mathrm{mM} \mathrm{NaCl}$ downstep, a concentration used previously to stimulate ASER and induce a robust excitatory AIB response ${ }^{17}$. Unexpectedly, I found that the excitatory response to $50 \mathrm{mM}$ $\mathrm{NaCl}$ downstep of the GluT KO mutants, regardless of class as proximal or distal transporters, all displayed more intense increase in GCaMP signals than WT (Figure 28). Disaggregation of AIB data also confirms that AIBR has less intense $\mathrm{Ca}^{2+}$ response than AIBL (Figure 28B, D), in line with previous observations by Rabinowitch et al. $(2021)^{15}$. The intensity of responses were relatively the same between GluT KO mutants; this may indicate that function of multiple GluTs contribute to clearance of ASEL->AIB synapses, even with the asymmetry in AIBL and AIBR responses. 
To determine whether GluT KO impacts clearance of inhibitory Glu signals and whether there any GluT displays preferential clearance of the distal neurite, I then performed experiments with an inhibitory $50 \mathrm{mM}$ upstep. While there was no significant difference in the drop in GCaMP intensity in the initial response to $\mathrm{NaCl}$ upstep (especially with the responses of WT and GluT KO mutants in AIBR, Figure 29D), the activity of AIBL neurons in all GluT KO animals were prolonged compared to WT (Figure 29A-C). This may indicate that all GluTs contribute to the clearance of ASEL->AIB synapses, and that loss of function for any GluT impairs clearance along the entire length of the AIB neurite. 

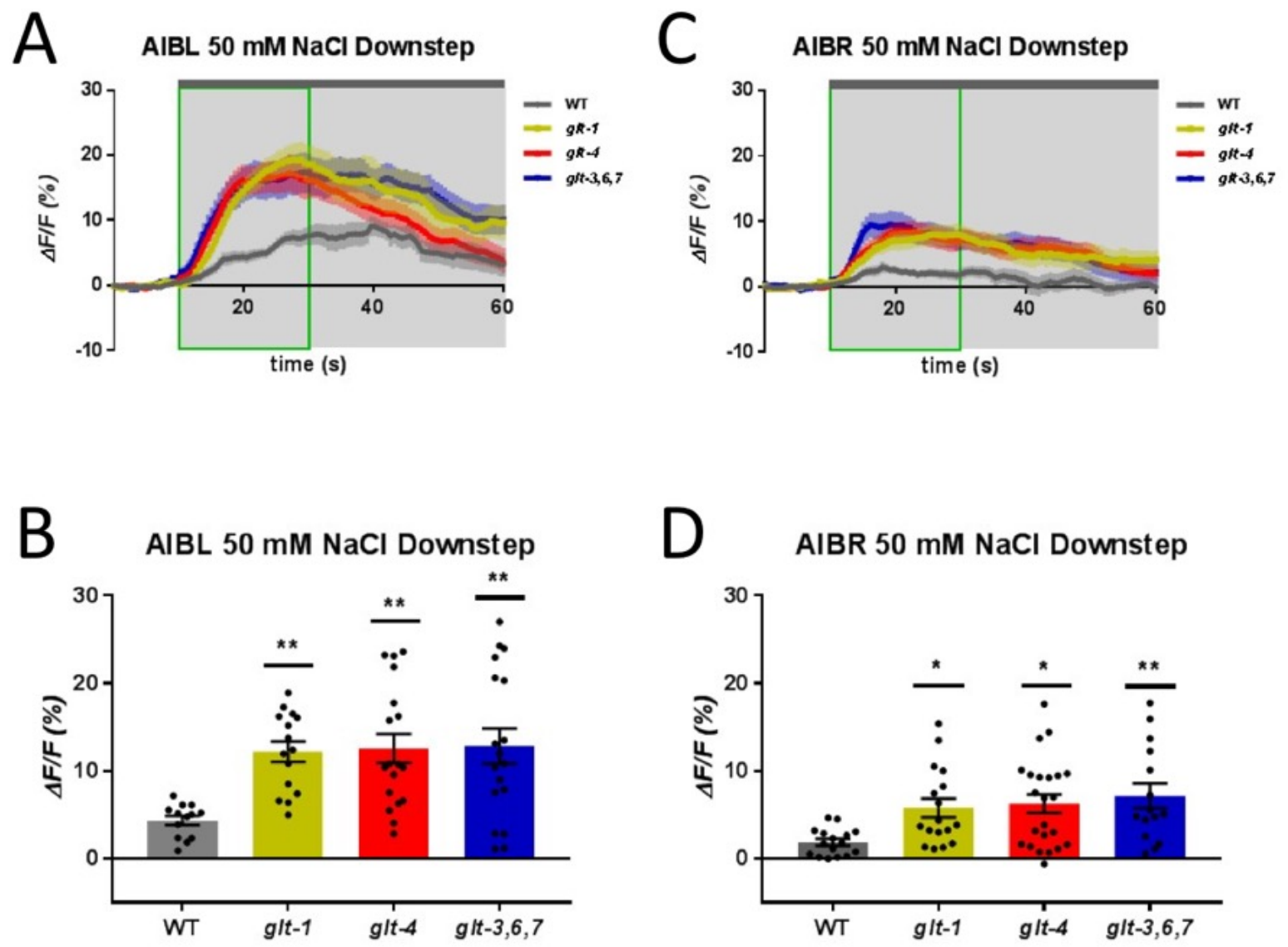

Figure 28. GluT KO mutants display heightened Glu-mediated responses of AIB to stimulation of sensory neuron ASER by a 50 mM NaCl downstep. WT and GluT KO animals expressing GCaMP6 in AIB neurons were imaged in the cell soma. Light gray shading indicates 50 s period of exposure to a $0 \mathrm{mM}$ $\mathrm{NaCl}$ solution after worms were acclimated to salt buffer with a $\mathrm{NaCl}$ concentration of $50 \mathrm{mM} \mathrm{NaCl}$. Exposure to salt buffer with $0 \mathrm{mM} \mathrm{NaCl}$ begins at $t=10 \mathrm{~s}$. The first $10 \mathrm{~s}$ of the recording was averaged to serve as the baseline fluorescence, $\mathrm{F}_{0}$. Green box indicates a window of $20 \mathrm{~s}$ where AIB responds to the initial change in $\mathrm{NaCl}$, for which the fluorescence change $(\Delta \mathrm{F})$ was averaged and presented in the bar graphs. Each data point on the bars for each strain represents the average fluorescent change during the $20 \mathrm{~s}$ window of the initial AIB response, $* P<0.05,{ }^{* *} P<0.01$, ANOVA with Bonferroni correction. Light colored shading around each curve and the error bars on bar graphs indicate SEM. $\mathrm{n}=11-17$ for each strain. 
A

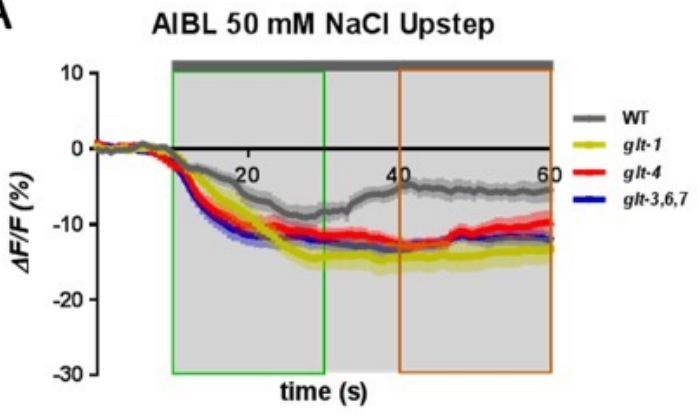

AIBL $50 \mathrm{mM} \mathrm{NaCl}$ Upstep

B

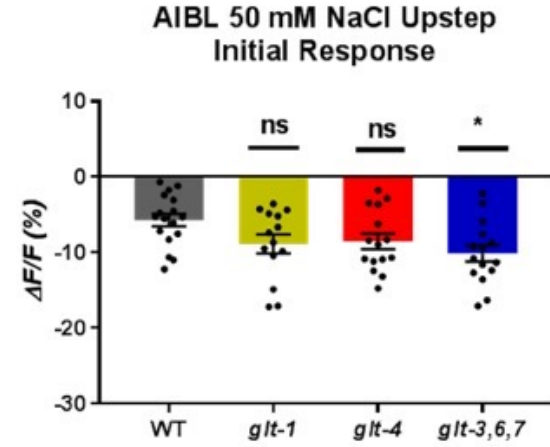

AIBL $50 \mathrm{mM}$ NaCl Upstep

C Latter Response

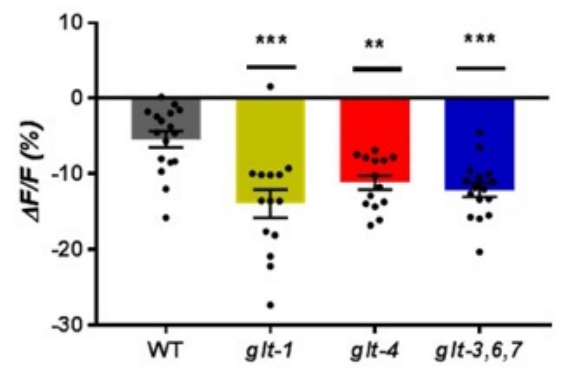

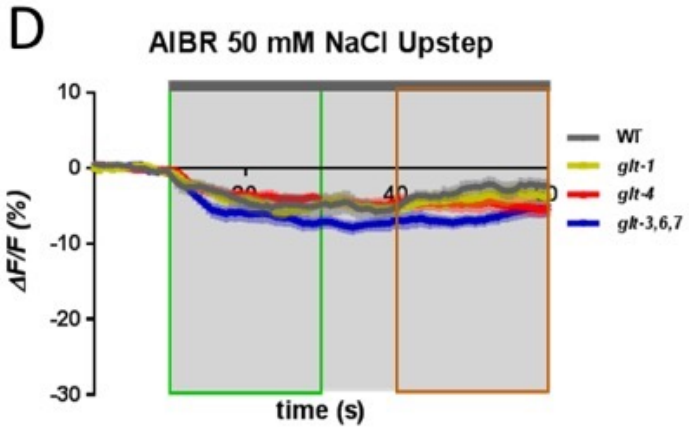

E

AIBR $50 \mathrm{mM} \mathrm{NaCl}$ Upstep Initial Response

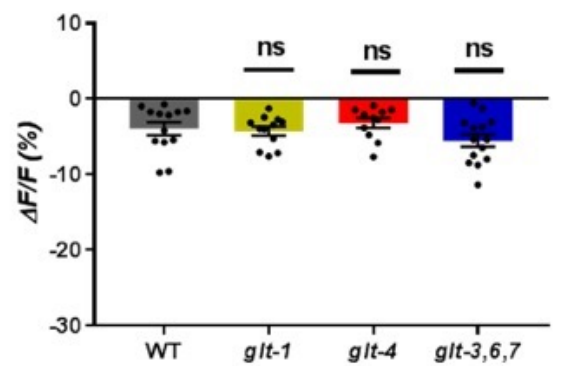

AIBR $50 \mathrm{mM} \mathrm{NaCl}$ Upstep

F

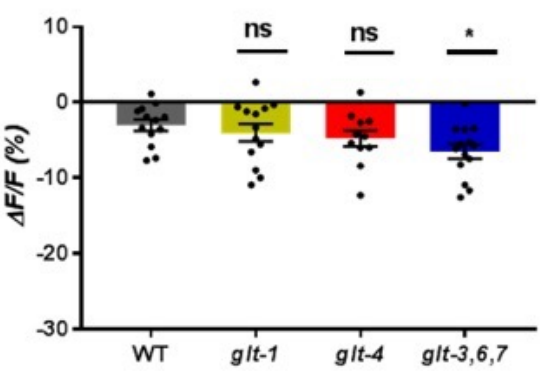

Figure 29. GluT KO mutants display prolonged and more intense Glu-mediated inhibition of AIB to stimulation of sensory neuron ASEL by a $50 \mathrm{mM} \mathrm{NaCl}$ upstep. WT and GluT KO animals expressing GCaMP6 in AIB neurons were imaged in the cell soma. Light gray shading indicates $50 \mathrm{~s}$ period of exposure to a $50 \mathrm{mM} \mathrm{NaCl}$ solution after worms were acclimated to salt buffer with a $\mathrm{NaCl}$ concentration of $0 \mathrm{mM} \mathrm{NaCl}$. Average traces of changes in GCaMP6 fluorescence and averaged steadystate responses to $50 \mathrm{mM} \mathrm{NaCl}$ downstep are analyzed similarly to those in Fig 28 for (B) and (E), with the additional analyses of the period within the brown box indicating a window of $20 \mathrm{~s}$ after the initial AIB response to change in $\mathrm{NaCl}$ concentration. The fluorescence change $(\Delta \mathrm{F})$ within the later time window was averaged and presented in the bar graphs $(\mathbf{C}, \mathbf{F})$. $* P<0.05, * P<0.01, * * * P<0.001, \mathrm{~ns}=$ not significant. ANOVA with Bonferroni correction. Light colored shading around each curve and the error bars on bar graphs indicate SEM. $\mathrm{n}=13-22$ for each strain. 


\subsubsection{Motility of the head and pharynx is critical to preserving the fidelity of AIB synaptic activity}

My previous experiments on the AVA circuit indicated that proximal GluTs GLT-1 and GLT-4 may have a privileged role in preserving signaling fidelity and preventing Glu signals from ASEL spilling over and ectopically exciting AVA ${ }^{21}$. Due to the proximity of ASE circuit synapses on the outer rim of the nerve ring to structures expressing GLT-1 and GLT-4, we had assumed simple, short-range diffusion from ASE synapses to be sufficient in delivering Glu for clearance by proximal GluTs, and that this diffusion is independent of the perfusion generated by mechanical pharyngeal pumping and head movements needed for vigorous clearance of Glu from inner nerve ring synapses ${ }^{21}$. To gauge the contributions of mechanical perfusion to clearance of AIB upon stimulation of ASEL or ASER, I imaged animals with GCaMP6 expressed in AIB that also contained extrachromosomal arrays for ectopic expression of Histamine-gated Chloride ( $\mathrm{HisCl})$ channels ${ }^{22}$. While I had previously used chemical paralytic agents to inhibit movement of pharyngeal and head muscles of the nematode, both tetramisole ${ }^{23,24}$ and $\mathrm{BDM}^{25,26}$ were found to have off-target effects that led to differing results in my imaging experiments of AIB (for example, notably, glt-4 animals still responded to $50 \mathrm{mM}$ $\mathrm{NaCl}$ upstep when paralyzed with tetramisole, whereas paralysis with BDM eliminated this response). An alternative mode of paralysis that does not have off-target effects is the expression $\mathrm{HisCl}$ channels on muscle cells; C. elegans do not have endogenous receptors for histamine, and histamine-induced paralysis eliminates the possibility of interfering with neuronal activity, and method has been demonstrated to be reliable, reversible, and tissue-specific ${ }^{22}$. I generated and injected constructs for expression of $\mathrm{HisCl}$ under specific muscle promoters: $P_{m y o-2}$ for expression in pharyngeal muscle, and $P_{m y o-3}$ for body wall muscle ${ }^{27,28}$. I found that in absence of 
pharyngeal pumping and local head movements, excitatory postsynaptic responses of AIB neurons to $50 \mathrm{mM} \mathrm{NaCl}$ upstep are lost (Figure 30B, E). This loss in response may be attributed to GluR desensitization arising from impaired synaptic Glu clearance. Alternatively, if following the model where perfusion and Glu released from other neurons is needed for buildup of Glu to reach high enough levels to induce a response in AIB, a loss in response may also indicate impaired diffusion of Glu the AIB-expressed Glu receptors. This trend is seen in AIBL and AIBR across all strains, hinting at a reliance on mechanical agitation and perfusion in facilitating synaptic clearance of ASER->AIBL Glu, even in WT animals. 
$50 \mathrm{mM} \mathrm{NaCl}$ Downstep Before Histamine Paralysis AIBL

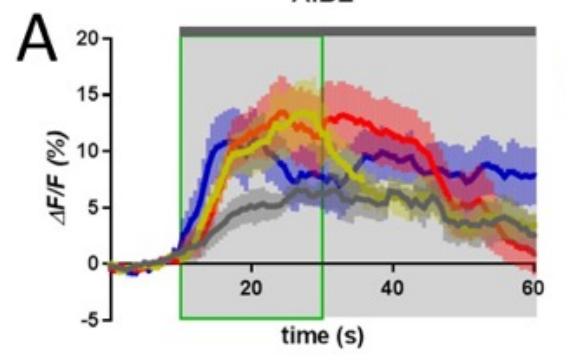

$50 \mathrm{mM}$ NaCl Downstep During Histamine Paralysis AIBL

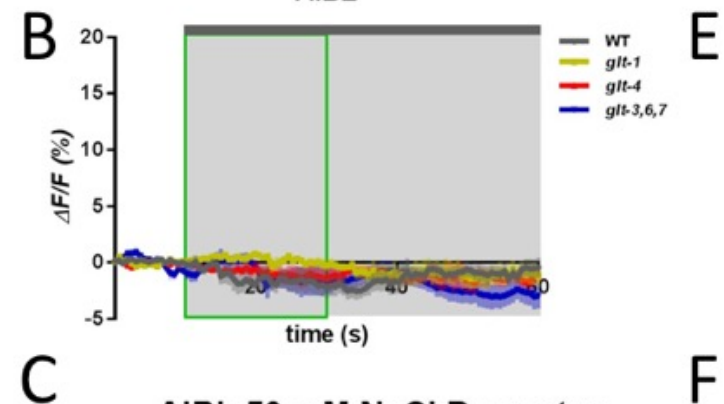

AIBL 50 mM NaCl Downstep

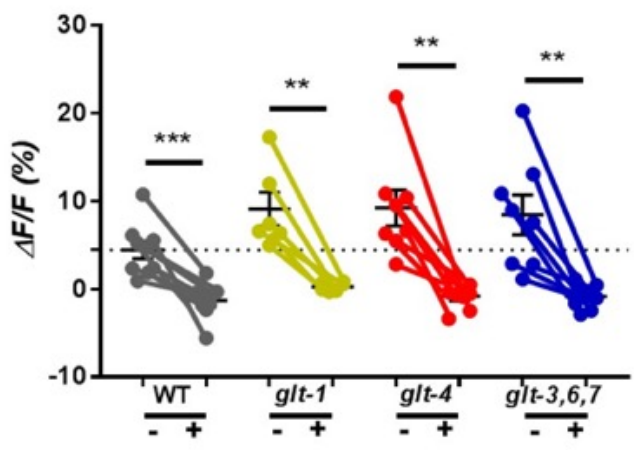

$50 \mathrm{mM} \mathrm{NaCl}$ Downstep Before Histamine Paralysis AIBR

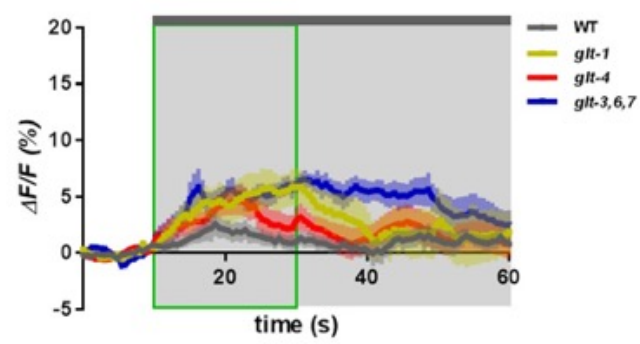

$50 \mathrm{mM} \mathrm{NaCl}$ Downstep During Histamine Paralysis AIBR

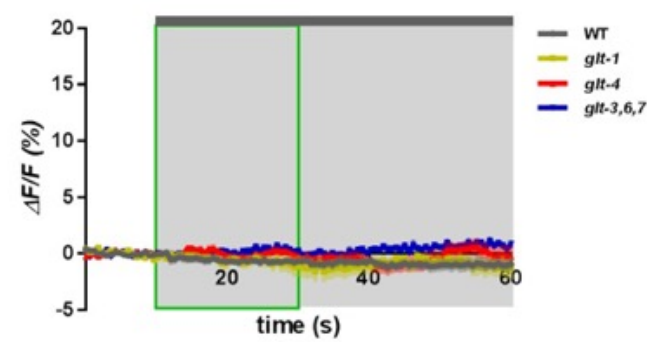

F

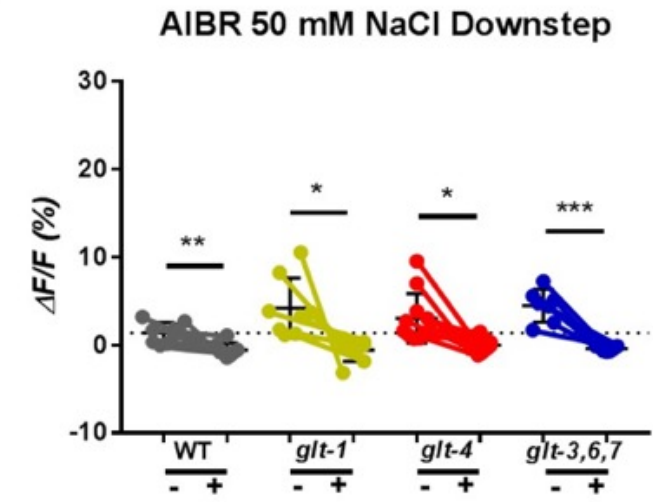

Figure 30. Paralysis eliminates excitatory AIB responses to $50 \mathrm{mM} \mathrm{NaCl}$ downstep. WT and GluT KO animals expressing GCaMP6 in AIB neurons were imaged in the cell soma. Light gray shading indicates $50 \mathrm{~s}$ period of exposure to a $0 \mathrm{mM} \mathrm{NaCl}$ solution after worms were acclimated to salt buffer with a $\mathrm{NaCl}$ concentration of $50 \mathrm{mM} \mathrm{NaCl}$. Average traces of changes in GCaMP6 fluorescence and averaged steady-state responses to $50 \mathrm{mM} \mathrm{NaCl}$ downstep are analyzed similarly to those in Fig 28. Average changes in fluorescence intensity during the $20 \mathrm{~s}$ window of stable state of stimulation before and after exposure to the paralytic agent are denoted in $(\mathbf{C})$ and $(\mathbf{F}){ }^{*} P<0.05, * * P<0.01, * * * P<$ 0.001, ANOVA with Bonferroni correction. Light colored shading around each curve and the error bars on bar graphs indicate SEM. $\mathrm{n}=6-11$ for each strain. Initial stimulation is shown in $(\mathbf{A}, \mathbf{D})$, while stimulation during paralysis is shown in $(\mathbf{B}, \mathbf{E})$.

Postsynaptic responses of AIB neurons to a $50 \mathrm{mM} \mathrm{NaCl}$ upstep are similarly impacted under paralysis, with the inhibition response seemingly lost upon restriction of pharyngeal pumping 
and local pharynx movements. While both AIB neurons display inhibitory Glu response to stimulation prior to paralysis, these $\mathrm{Ca}^{2+}$ responses are lost when the animal is paralyzed, which may result from desensitization of inhibitory GluRs arising from impaired synaptic Glu clearance (Figure 31). This trend is seen in AIBL and AIBR across animals regardless of GluT function, indicative of the strong roles of mechanical agitation and perfusion in facilitating synaptic clearance of ASEL->AIBL Glu. 
$50 \mathrm{mM} \mathrm{NaCl}$ Upstep Before Histamine Paralysis AIBL

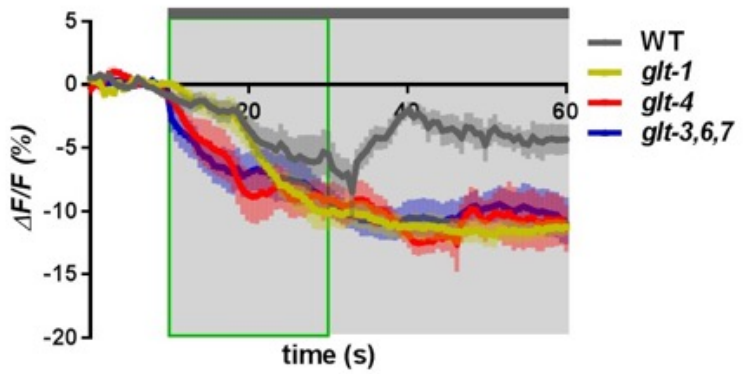

$50 \mathrm{mM} \mathrm{NaCl}$ Upstep During Histamine Paralysis AIBL

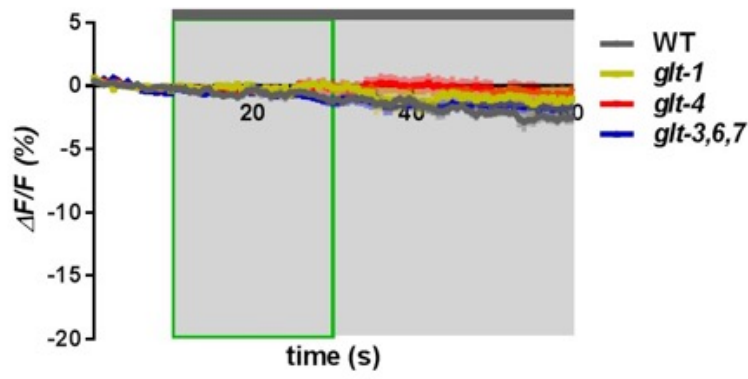

AIBL $50 \mathrm{mM} \mathrm{NaCl}$ Upstep

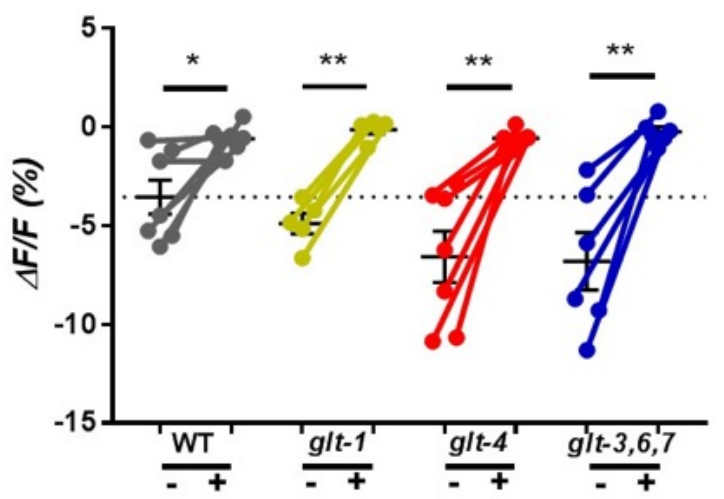

$50 \mathrm{mM} \mathrm{NaCl}$ Upstep Before Histamine Paralysis AIBR

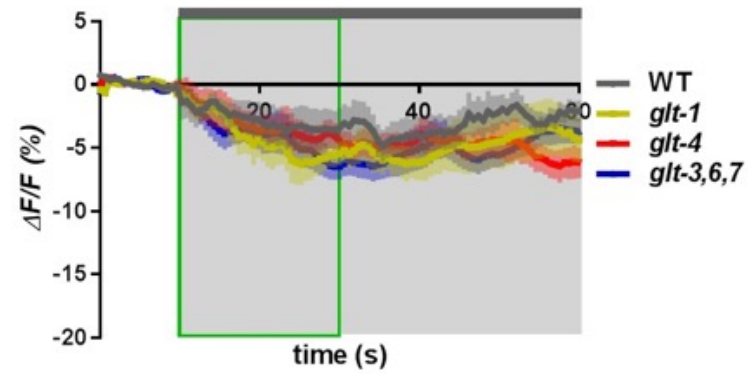

$50 \mathrm{mM} \mathrm{NaCl}$ Upstep During Histamine Paralysis AIBR

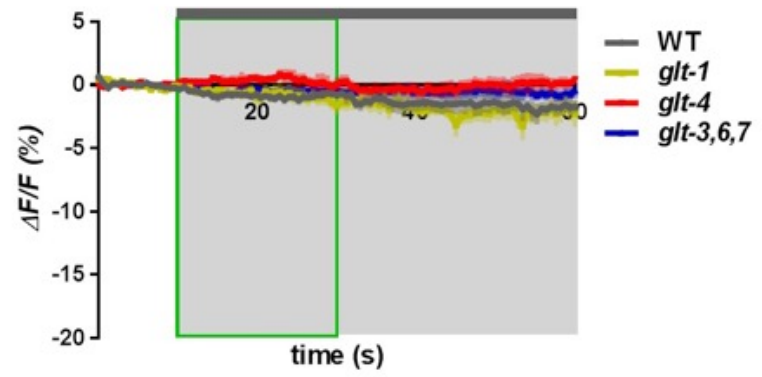

AIBR 50 mM NaCI Upstep

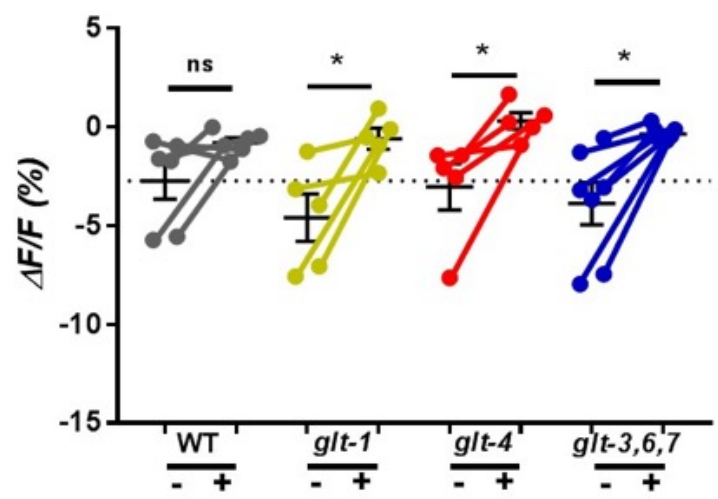

Figure 31. Paralysis eliminates AIB inhibition response to $50 \mathrm{mM}$ NaCl upstep. WT and GluT KO animals expressing GCaMP6 in AIB neurons were imaged in the cell soma. Light gray shading indicates $50 \mathrm{~s}$ period of exposure to a $50 \mathrm{mM} \mathrm{NaCl}$ solution after worms were acclimated to salt buffer with a $\mathrm{NaCl}$ concentration of $0 \mathrm{mM} \mathrm{NaCl}$. Average traces of changes in GCaMP6 fluorescence and averaged steadystate responses to $50 \mathrm{mM} \mathrm{NaCl}$ upstep are analyzed similarly to those in Fig 28. Average changes in fluorescence intensity during the $20 \mathrm{~s}$ window of stable state of stimulation before and after exposure to the paralytic agent are denoted in $(\mathbf{C})$ and $(\mathbf{F}) .{ }^{*} P<0.05, * * P<0.01$, ANOVA with Bonferroni correction. Light colored shading around each curve and the error bars on bar graphs indicate SEM. $\mathrm{n}=$ 5-7 for each strain. Initial stimulation is shown in (A, D), while stimulation during paralysis is shown in (B, E). 
Subsequently, I performed upstep and downstep stimulation experiments with $1 \mathrm{mM} \mathrm{NaCl}$. My previous experiments had revealed ectopic AVA responses during ASEL response to $1 \mathrm{mM}$ $\mathrm{NaCl}$ upstep in proximal GluT mutants, likely a result if spillover (Figure 11). Drop assays also seemed to indicate that $1 \mathrm{mM} \mathrm{NaCl}$ is unusually an extremely repulsive concentration, with a higher fraction of $g l t-1$ and $g l t-4 \mathrm{KO}$ mutants displaying avoidance (reversal) responses, even when compared to higher yet chemoattractive $\mathrm{NaCl}$ concentrations (as seen previously in Figure 10). We wondered if there is something unique about this concentration and whether this higher rate of avoidance is somehow optimal for inducing AIB excitation, perhaps via Glu spillover from ASEL onto GLR-1-expressing proximal neurites. However, excitatory AIB responses are less intense in response to a downstep of $1 \mathrm{mM}$ as opposed to $50 \mathrm{mM} \mathrm{NaCl}$, and are still largely masked or eliminated when head movement and pharyngeal pumping is eliminated under histamine-induced paralysis (Figure 32). I also observed that AIB inhibitory responses are less intense (or barely detectable) to an upstep of $1 \mathrm{mM}$, and are nearly indistinguishable from $\mathrm{Ca}^{2+}$ responses under paralysis conditions (Figure 33). Overall, I found that the intensity of excitatory and inhibitory $\mathrm{AIB}$ responses appear to scale with $\mathrm{NaCl}$ concentration, since the magnitude to responses to $1 \mathrm{mM} \mathrm{NaCl}$ changes are smaller compared to responses seen previously with 50 $\mathrm{mM} \mathrm{NaCl}$ stimulation (Figures 28-31). 


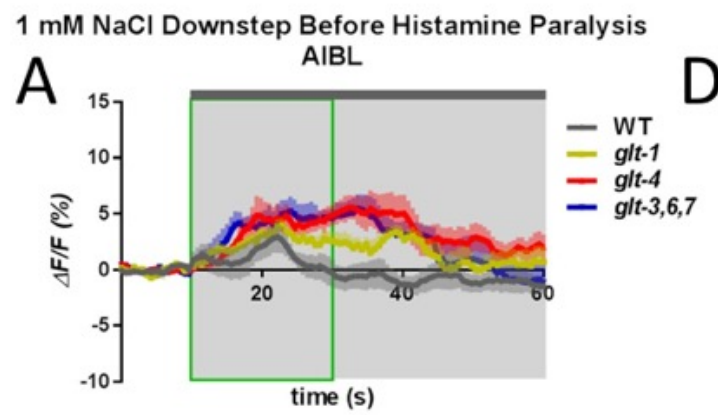

$1 \mathrm{mM} \mathrm{NaCl}$ Downstep During Histamine Paralysis B

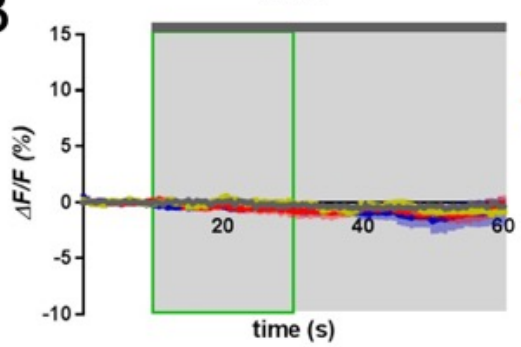

C

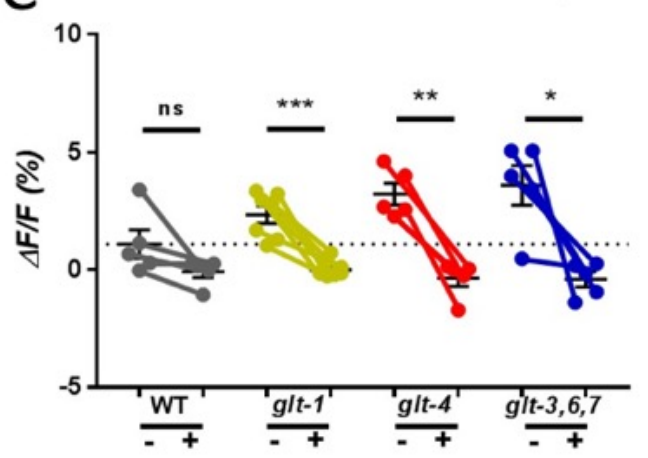

F
$1 \mathrm{mM} \mathrm{NaCl}$ Downstep Before Histamine Paralysis AIBR

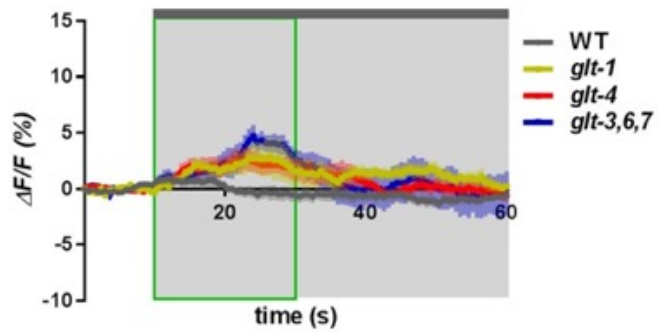

$1 \mathrm{mM} \mathrm{NaCl}$ Downstep During Histamine Paralysis

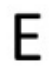

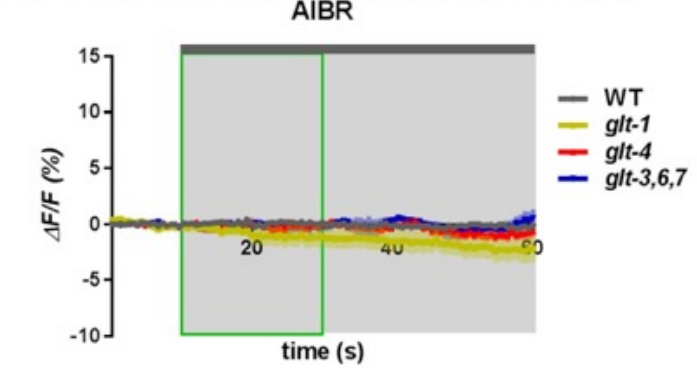

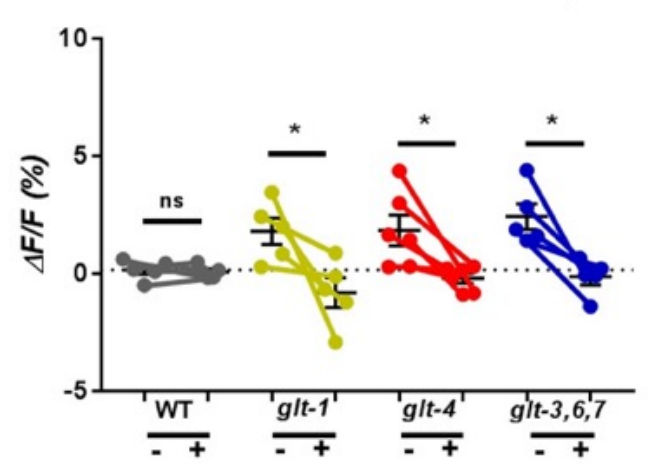

Figure 32. Paralysis eliminates excitatory AIB responses to 1 mM NaCl downstep. WT and GluT KO animals expressing GCaMP6 in AIB neurons were imaged in the cell soma. Light gray shading indicates $50 \mathrm{~s}$ period of exposure to a $0 \mathrm{mM} \mathrm{NaCl}$ solution after worms were acclimated to salt buffer with a $\mathrm{NaCl}$ concentration of $1 \mathrm{mM} \mathrm{NaCl}$. Average traces of changes in GCaMP6 fluorescence and averaged steadystate responses to $1 \mathrm{mM} \mathrm{NaCl}$ downstep are analyzed similarly to those in Fig 28. Average changes in fluorescence intensity during the $20 \mathrm{~s}$ window of stable state of stimulation before and after exposure to the paralytic agent are denoted in $(\mathbf{C})$ and $(\mathbf{F}) . * P<0.05, * * P<0.01$, $* * * P<0.001$, ANOVA with Bonferroni correction. Light colored shading around each curve and the error bars on bar graphs indicate SEM. $n=5-7$ for each strain. Initial stimulation is shown in $(\mathbf{A}, \mathbf{D})$, while stimulation during paralysis is shown in $(\mathbf{B}, \mathbf{E})$. 

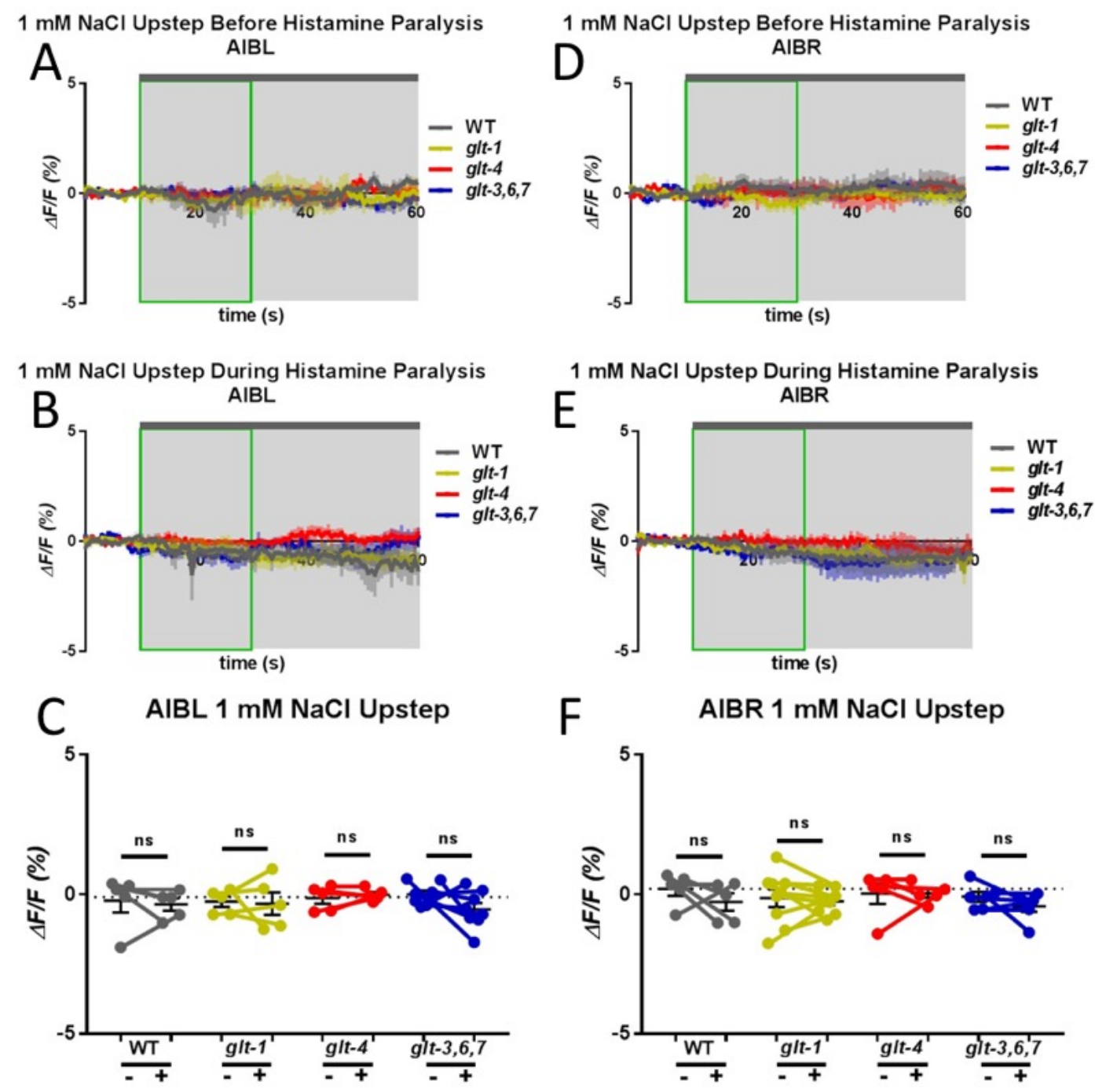

Figure 33. Paralysis eliminates AIB inhibition responses to $1 \mathbf{m M ~ N a C l}$ upstep. WT and GluT KO animals expressing GCaMP6 in AIB neurons were imaged in the cell soma. Light gray shading indicates $50 \mathrm{~s}$ period of exposure to a $1 \mathrm{mM} \mathrm{NaCl}$ solution after worms were acclimated to salt buffer with a $\mathrm{NaCl}$ concentration of $0 \mathrm{mM} \mathrm{NaCl}$. Average traces of changes in GCaMP6 fluorescence and averaged steadystate responses to $1 \mathrm{mM} \mathrm{NaCl}$ upstep are analyzed similarly to those in Fig 28. Average changes in fluorescence intensity during the $20 \mathrm{~s}$ window of stable state of stimulation before and after exposure to the paralytic agent are denoted in (C) and (F). ANOVA with Bonferroni correction. Light colored shading around each curve and the error bars on bar graphs indicate SEM. $n=5-9$ for each strain. Initial stimulation is shown in $(\mathbf{A}, \mathbf{D})$, while stimulation during paralysis is shown in $(\mathbf{B}, \mathbf{E})$.

\subsubsection{GCaMP recordings display little to no impact of GluT KO on local activity of AIB}

neurites. The somatic $\mathrm{Ca}^{2+}$ recordings reveal that GluTs, regardless of localization from the nematode nerve ring, all play a role in synaptic Glu clearance of the AIB neurons. To determine whether distinct localization of Glu signals occurs in response to changes in $\mathrm{NaCl}$ levels, (as 
postulated by Kuramochi and Doi, 2019), I recorded GCaMP signals of proximal and distal AIB neurites in response to excitatory downstep and inhibitory upstep of $50 \mathrm{mM} \mathrm{NaCl} . P_{n p r-9}:: g l r-1$ $m$ Cherry and $P_{n p r-9:: e a t-4 c D N A-m T a g B F P 2}$ constructs expressed in these animals allowed for distinction between the proximal/postsynaptic and distal/presynaptic regions, respectively (Figure 34). In AIB neurons, localized excitation is more discernable in the proximal, GLR-1 expressing neurites compared to distal neurites (Figures $35 \&$ 36), which may be indicative of localized Glu release from ASER onto AIB post-synaptic regions. These proximal excitatory responses are also more prominent in AIBL, consistent with previous observations of AIBL neurons exhibiting stronger somatic signals compared to AIBR.

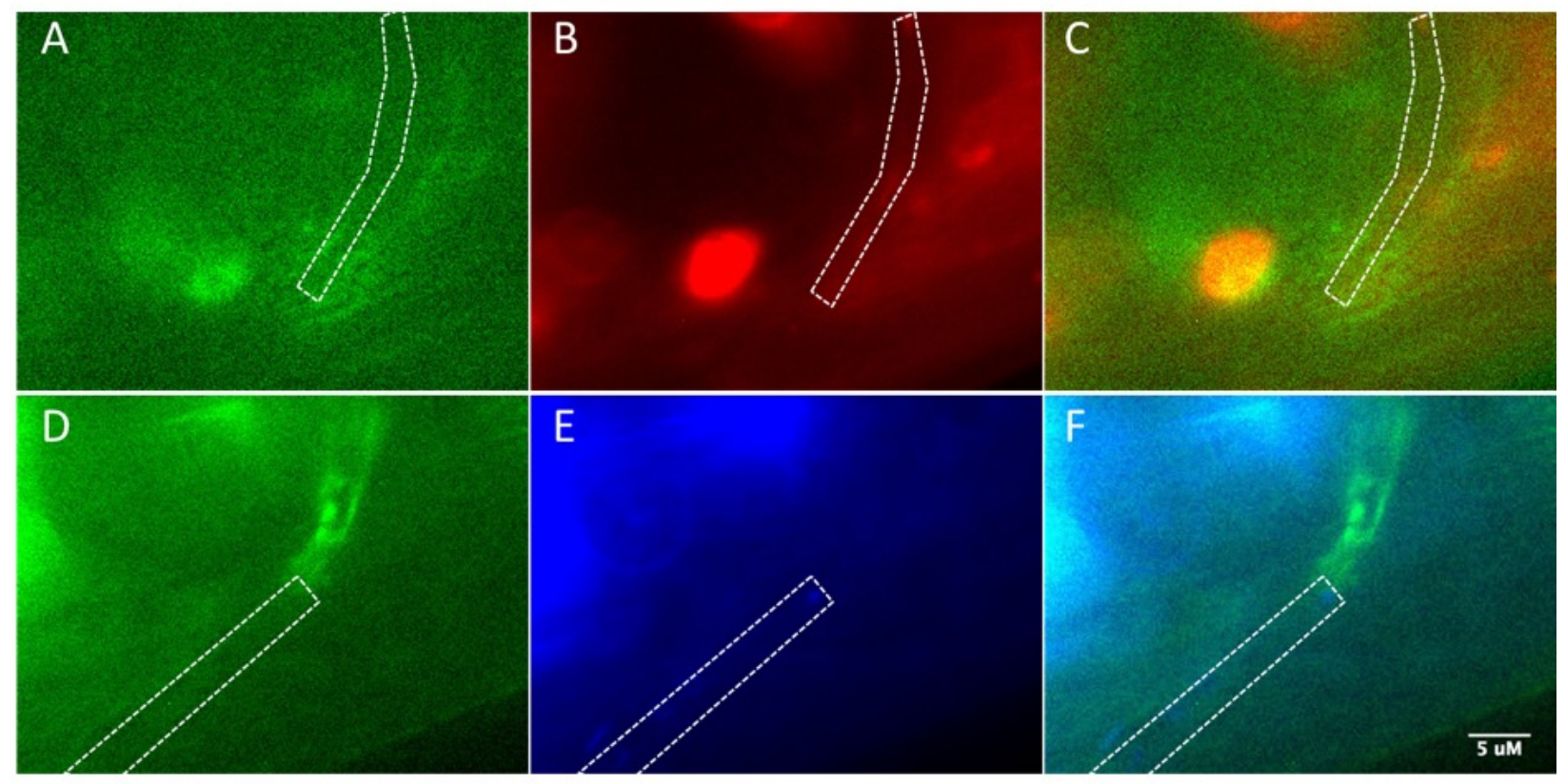

Figure 34. Specific markers allow for distinction between proximal and distal AIB neurites. GCaMP recordings from are captured from locations where GLR-1::mCherry (B) and EAT-4::mTagBFP2 (E) co-localize at the proximal/post-synaptic (C) and distal/pre-synaptic (F) locations of AIB neuronal processes, respectively. Dotted white outlines highlight the proximal (A) or distal (D) AIB neurites, indicated by GCaMP6. While GLR$1::$ mCherry is expressed more diffusely on proximal AIB neuronal processes and soma, EAT-2::mTagBFP2 is highly punctate and localized to distal neuronal processes. All fluorescent reporters are expressed under the AIBspecific promoter Pnpr-9. 
Compared to excitatory stimulation responses, GCaMP responses to inhibitory stimulation with a $50 \mathrm{mM} \mathrm{NaCl}$ upstep are harder to discern in the neurites. Minute inhibition can be seen in both proximal and distal neurite regions in AIBL (Figure 37) and AIBR (Figure 38). Inhibition responses overall appear slightly stronger in AIBL compared to AIBR, particularly in the distal neurites. However, these results are preliminary and inconclusive due to the low resolution of GCaMP6 signals at the neurites. Repeating these experiments with stronger neurite iGluSnFR or newer versions of GCaMP may yield more conclusive results or validate the GCaMP data. 
A

$50 \mathrm{mM} \mathrm{NaCl}$ Downstep

AIBL proximal neurites
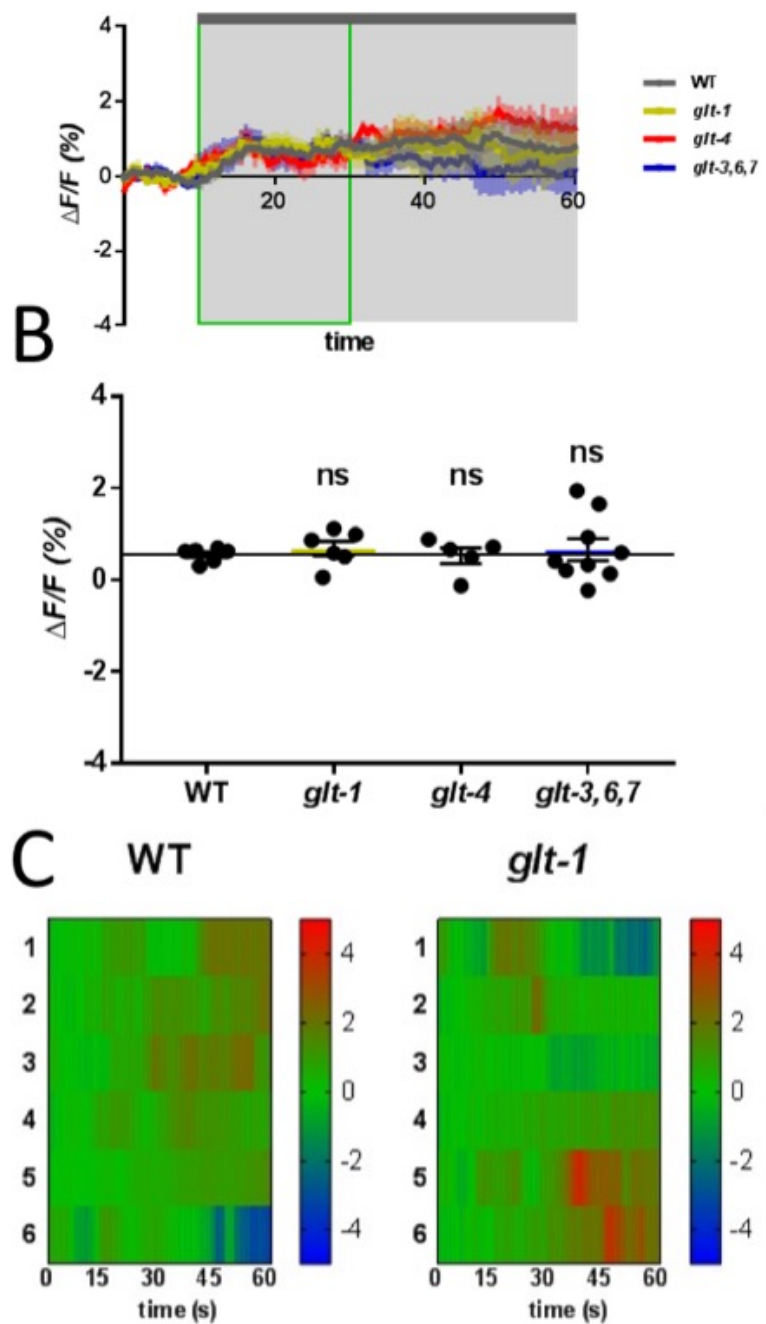

glt-4

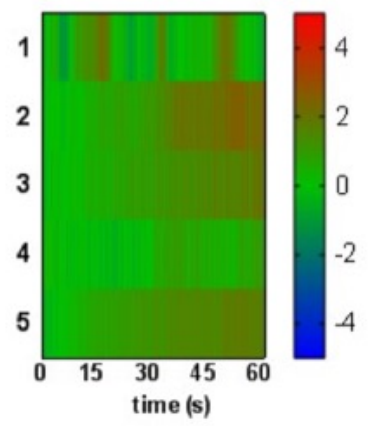

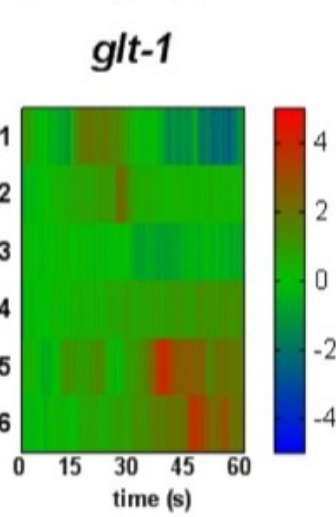

glt-3,6,7

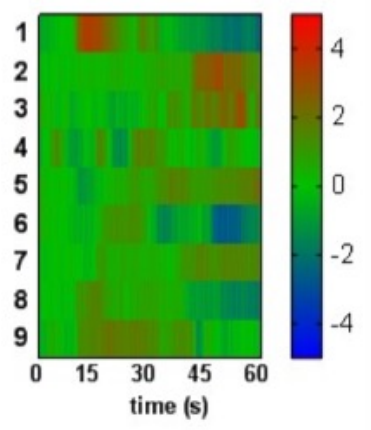

D

$50 \mathrm{mM} \mathrm{NaCl}$ Downstep

AIBL proximal neurites
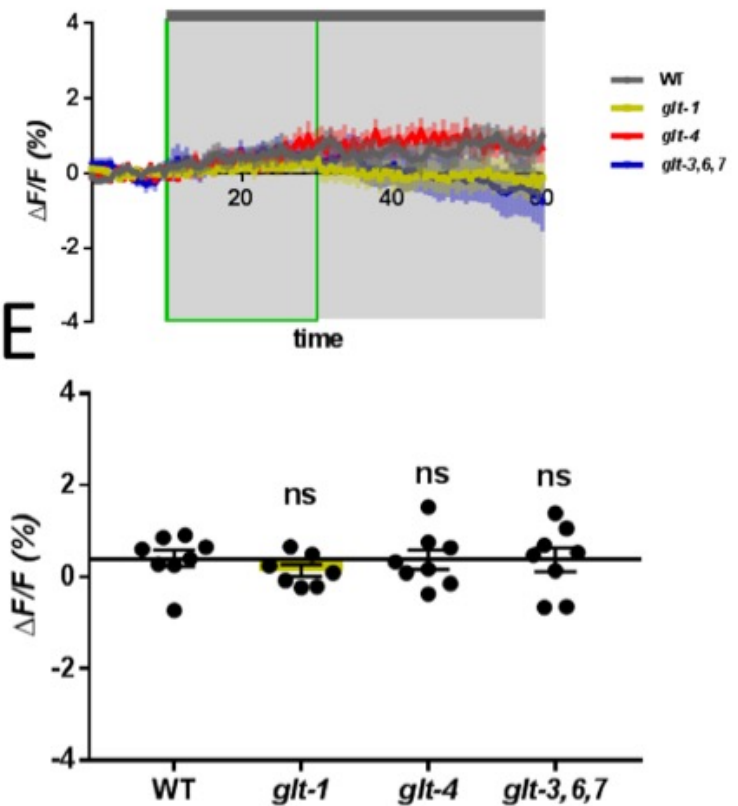

F WT

glt-1
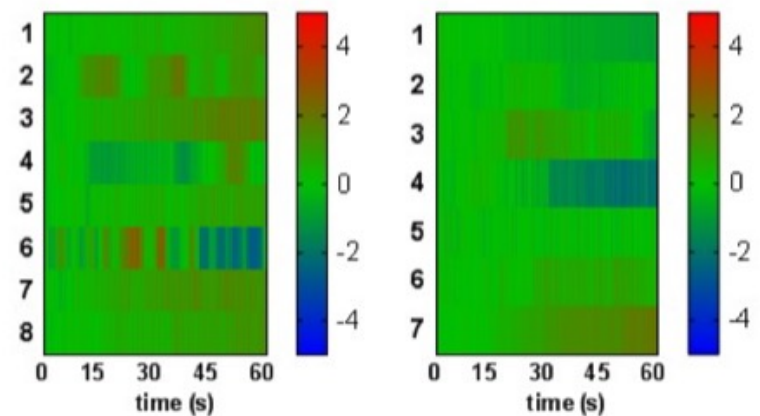

glt-4

glt-3,6,7
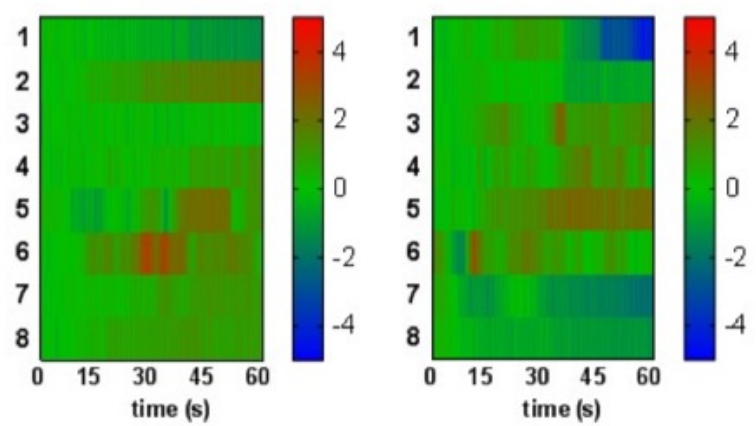
Figure 35. GluT KO mutants do not display significantly different $\mathrm{Ca}^{2+}$ responses in proximal AIB neurites to an excitatory $50 \mathrm{mM} \mathbf{~ N a C l}$ downstep. WT and GluT KO animals expressing GCaMP6 in AIB neurons were imaged at proximal neurites. Light gray shading indicates $50 \mathrm{~s}$ period of exposure to a $0 \mathrm{mM} \mathrm{NaCl}$ solution after worms were acclimated to salt buffer with a $\mathrm{NaCl}$ concentration of $50 \mathrm{mM}$ $\mathrm{NaCl}$. Average traces of changes in GCaMP6 fluorescence and averaged steady-state responses to $50 \mathrm{mM}$ $\mathrm{NaCl}$ downstep are analyzed similarly to those in Fig 28 . Average changes in fluorescence intensity during the $20 \mathrm{~s}$ window of stable state of stimulation are denoted in (B) and (E). Individual recordings from each worm are depicted as heat maps in $(\mathbf{C})$ and $(\mathbf{F})$. ANOVA with Bonferroni correction. Light colored shading around each curve and the error bars on bar graphs indicate SEM. $n=5-9$ for each strain. 

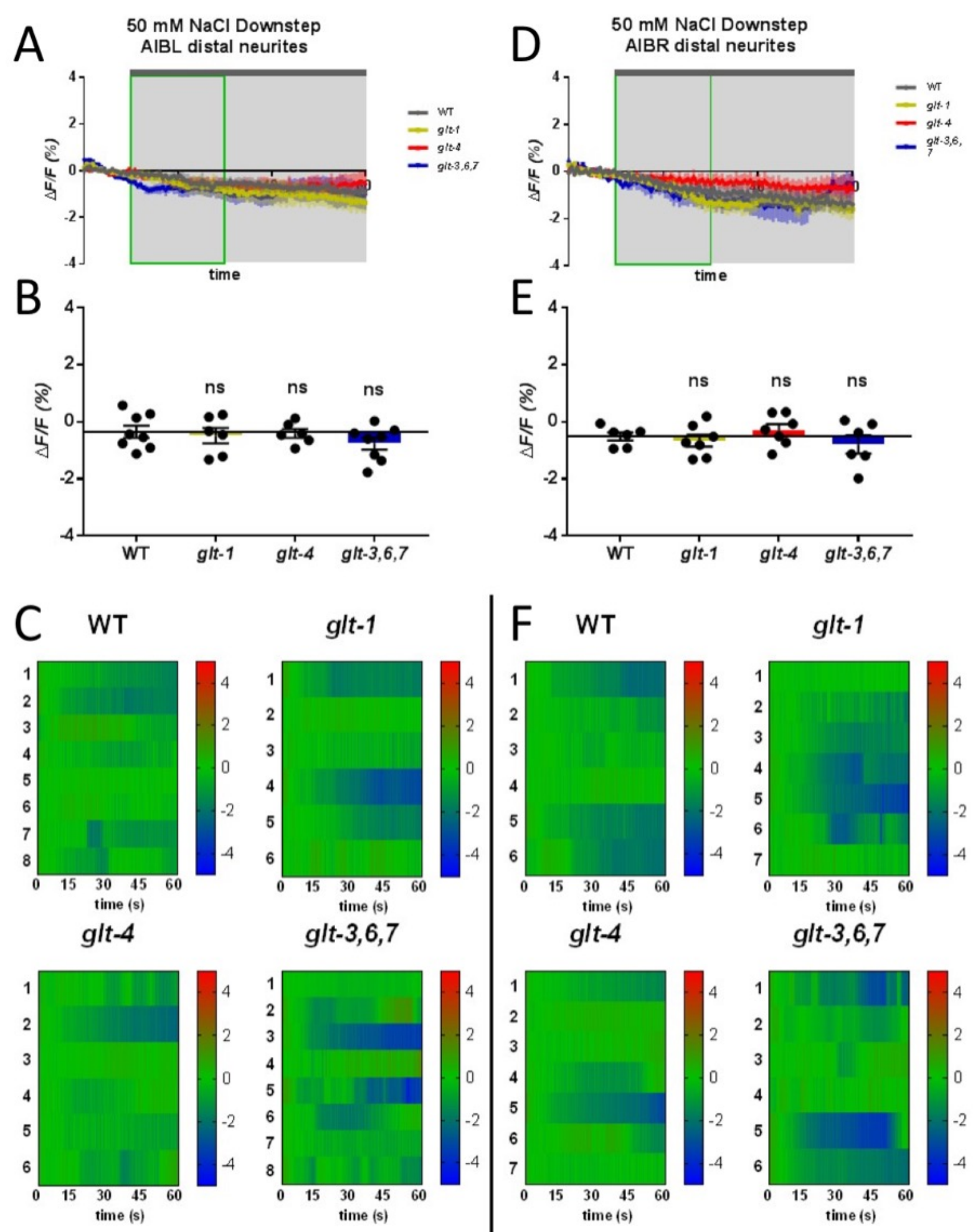

glt-3,6,7

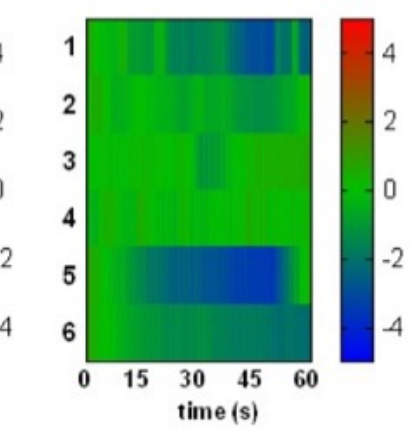


Figure 36. GluT KO mutants do not display significantly different $\mathrm{Ca}^{2+}$ responses in distal AIB neurites to an excitatory $50 \mathrm{mM}$ NaCl downstep. WT and GluT KO animals expressing GCaMP6 in AIB neurons were imaged at distal neurites. Light gray shading indicates $50 \mathrm{~s}$ period of exposure to a 0 $\mathrm{mM} \mathrm{NaCl}$ solution after worms were acclimated to salt buffer with a $\mathrm{NaCl}$ concentration of $50 \mathrm{mM} \mathrm{NaCl}$. Average traces of changes in GCaMP6 fluorescence and averaged steady-state responses to $50 \mathrm{mM} \mathrm{NaCl}$ downstep are analyzed similarly to those in Fig 28. Average changes in fluorescence intensity during the $20 \mathrm{~s}$ window of stable state of stimulation are denoted in (B) and (E). Individual recordings from each worm are depicted as heat maps in $(\mathbf{C})$ and $(\mathbf{F})$. ANOVA with Bonferroni correction. Light colored shading around each curve and the error bars on bar graphs indicate SEM. $n=6-8$ for each strain. 
A $\quad \begin{aligned} & 50 \mathrm{mM} \mathrm{NaCl} \text { Upstep } \\ & \text { AIBL proximal neurites }\end{aligned}$
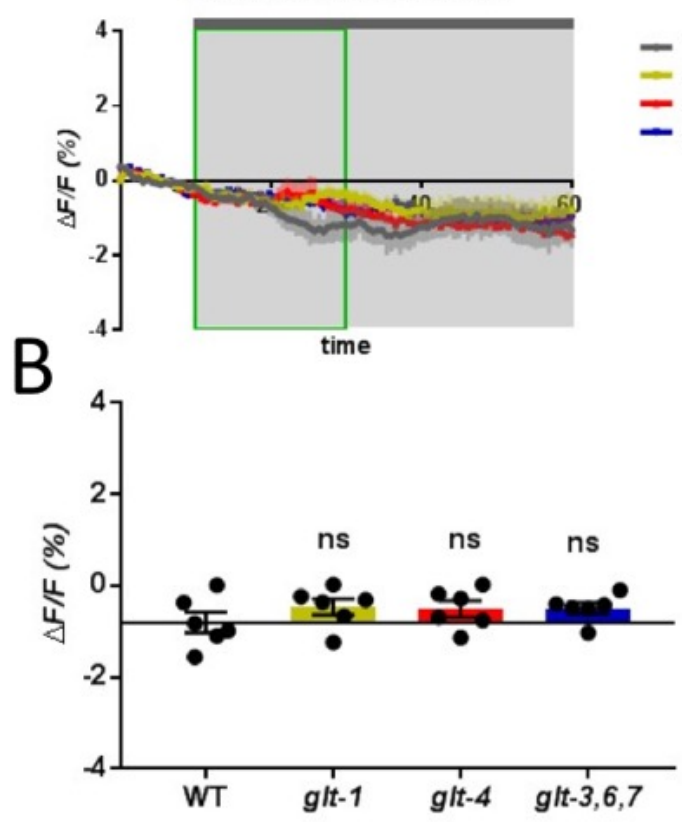

D $\quad \begin{aligned} & 50 \mathrm{mM} \mathrm{NaCl} \text { Upstep } \\ & \text { AlBR proximal neurites }\end{aligned}$
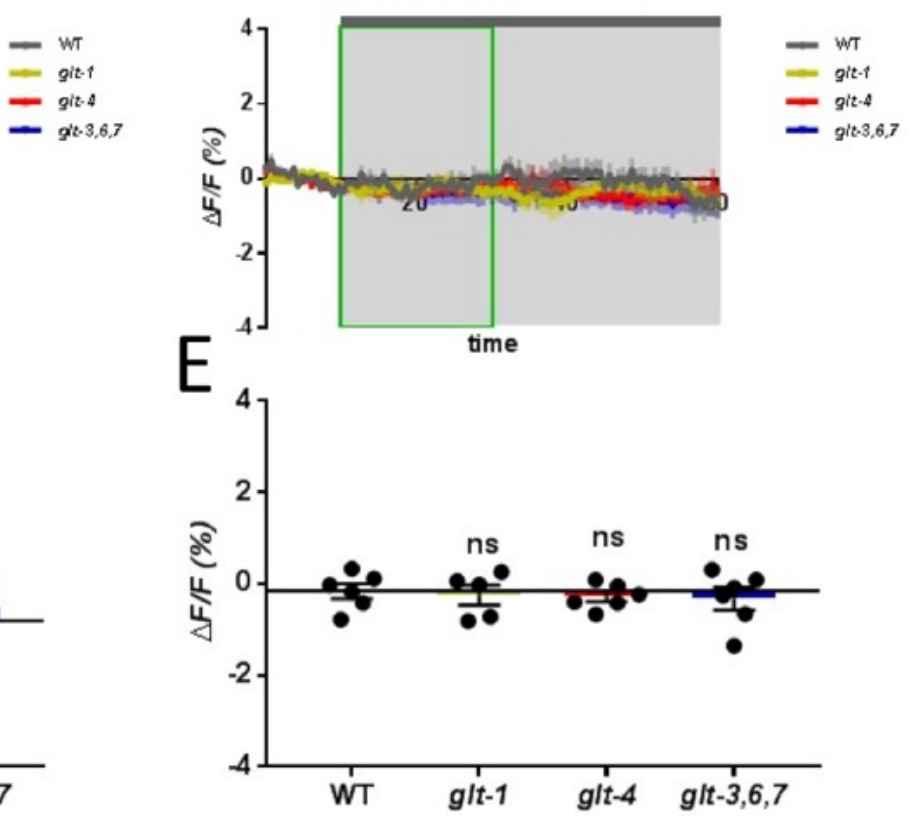

C WT
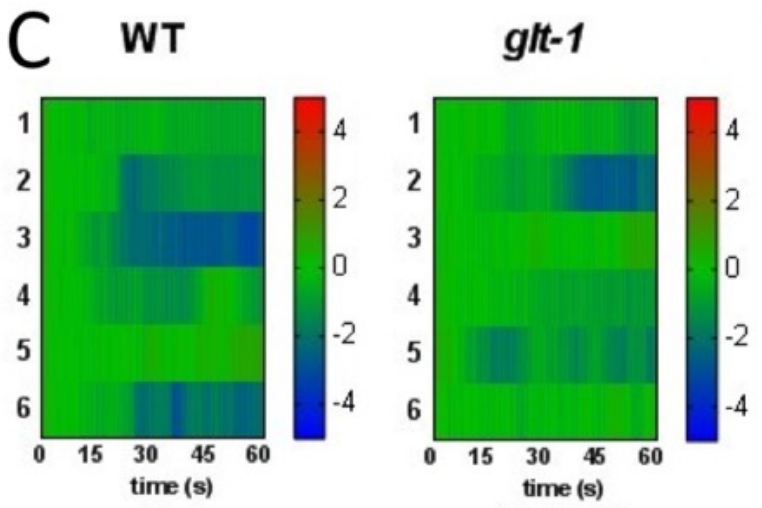

git-4

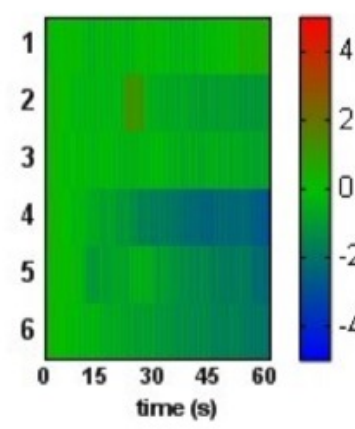
glt-3,6,7

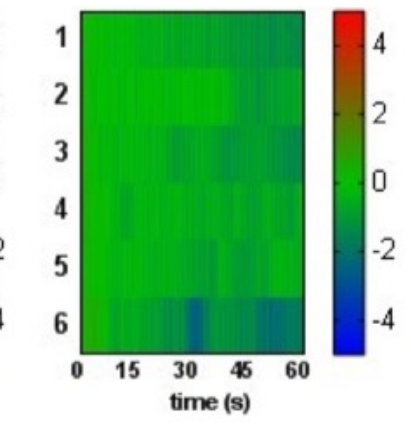

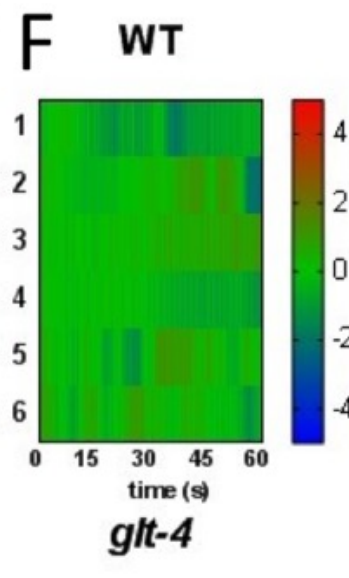
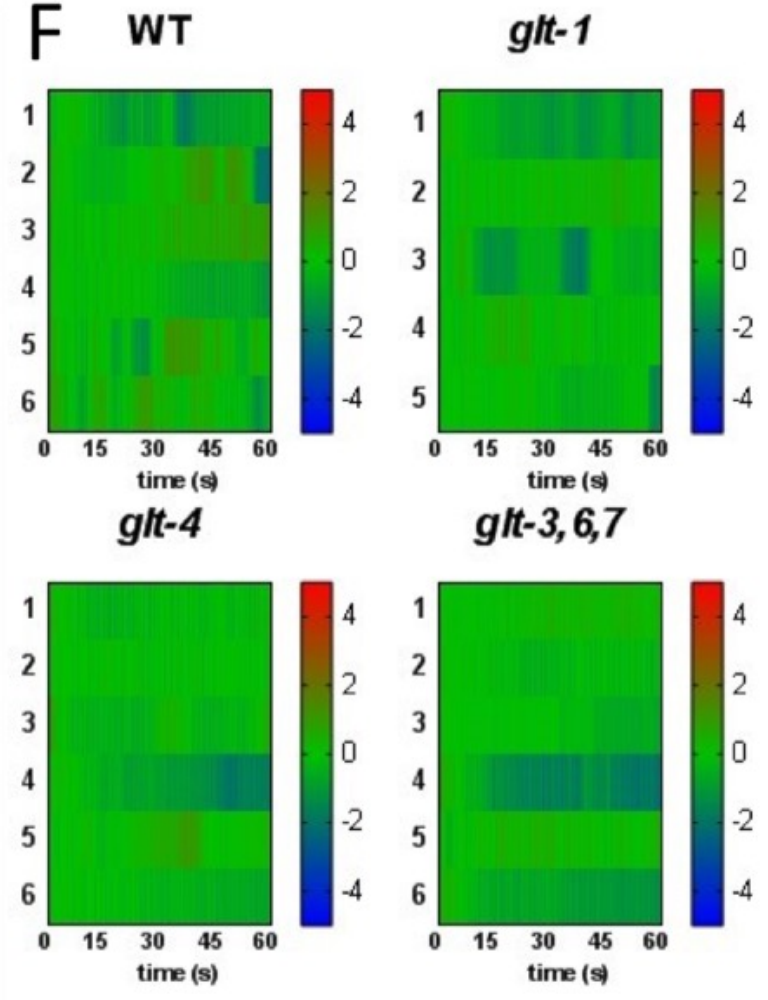
Figure 37. GluT KO mutants do not display significantly different $\mathrm{Ca}^{2+}$ responses in proximal AIB neurites to an inhibitory $50 \mathrm{mM} \mathrm{NaCl}$ upstep. WT and GluT KO animals expressing GCaMP6 in AIB neurons were imaged at proximal neurites. Light gray shading indicates $50 \mathrm{~s}$ period of exposure to a 50 $\mathrm{mM} \mathrm{NaCl}$ solution after worms were acclimated to salt buffer with a $\mathrm{NaCl}$ concentration of $0 \mathrm{mM} \mathrm{NaCl}$. Average traces of changes in GCaMP6 fluorescence and averaged steady-state responses to $50 \mathrm{mM} \mathrm{NaCl}$ upstep are analyzed similarly to those in Fig 28 . Average changes in fluorescence intensity during the 20 $\mathrm{s}$ window of stable state of stimulation are denoted in $\mathbf{( B )}$ and $(\mathbf{E})$. Individual recordings from each worm are depicted as heat maps in $(\mathbf{C})$ and $(\mathbf{F})$. ANOVA with Bonferroni correction. Light colored shading around each curve and the error bars on bar graphs indicate SEM. $n=5-6$ for each strain. 

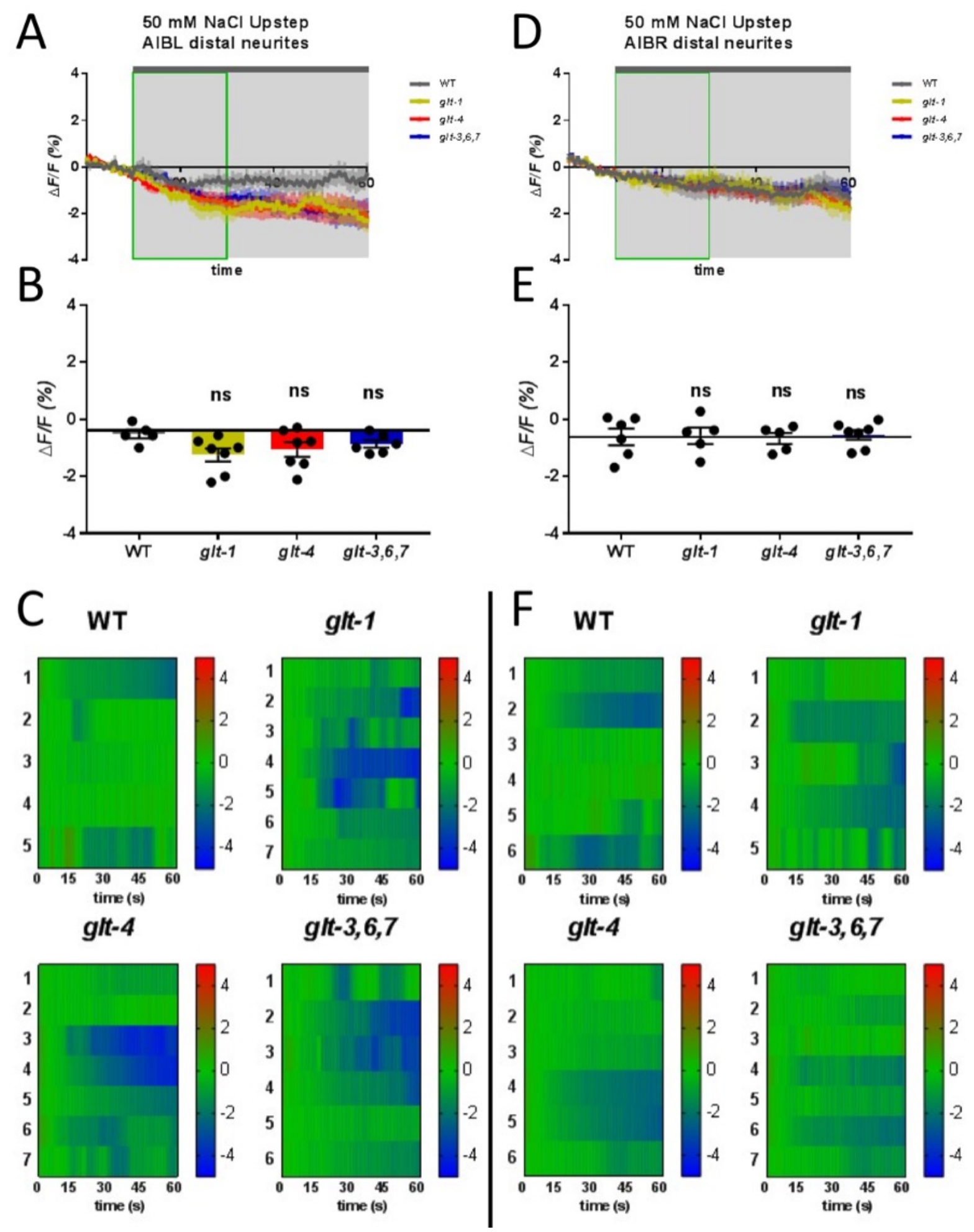
Figure 38. GluT KO mutants do not display significantly different $\mathrm{Ca}^{2+}$ responses in distal AIB neurites to an inhibitory $50 \mathrm{mM} \mathrm{NaCl}$ upstep. WT and GluT KO animals expressing GCaMP6 in AIB neurons were imaged at distal neurites. Light gray shading indicates $50 \mathrm{~s}$ period of exposure to a $50 \mathrm{mM}$ $\mathrm{NaCl}$ solution after worms were acclimated to salt buffer with a $\mathrm{NaCl}$ concentration of $0 \mathrm{mM} \mathrm{NaCl}$. Average traces of changes in GCaMP6 fluorescence and averaged steady-state responses to $50 \mathrm{mM} \mathrm{NaCl}$ upstep are analyzed similarly to those in Fig 28 . Average changes in fluorescence intensity during the 20 $\mathrm{s}$ window of stable state of stimulation are denoted in $\mathbf{( B )}$ and $\mathbf{( E )}$. Individual recordings from each worm are depicted as heat maps in $(\mathbf{C})$ and $(\mathbf{F})$. ANOVA with Bonferroni correction. Light colored shading around each curve and the error bars on bar graphs indicate SEM. $n=5-7$ for each strain.

\subsection{Discussion and Conclusion}

While glia have long been the canonical answer for how signal fidelity is maintained between mammalian Glu circuits, the AIB neuron in C. elegans (with its synapses with the ASE neurons) presents the unique question of how Glu neurotransmission is targeted to specific regions within the same neuron to mediate opposite responses to minute changes in salt concentration. We further explore the relationship between the pairs of ASE and AIB neurons, all which display asymmetry in activity, in mediating rapid, fine-tuned responses to changes in environmental $\mathrm{NaCl}$ levels.

Overall, the critical roles of pharyngeal pumping and head movements also extend to the clearance of Glu from ASE->AIB synapses. This is surprising given that the synapses of the ASE circuit neurons are localized to the outer rim of the nematode nerve ring, located closer to proximal (GLT-1 and GLT-4) GluT-expressing structures like the head muscle and hypodermis; and unlike neurons with synapses localized to the inner rim (like the members of the ASH circuit), neurons of the ASE circuit are not subject to washout by interstitial fluids agitated by pharyngeal motions (Figure 26). At first glance, this configuration appears more conducive to simple diffusion to GLT-1 and GLT-4 expressing structures for Glu clearance, rather than the replacement of Glu-rich synaptic fluid mediated by perfusion and bulk flow that allows for Glu 
transport to distal (GLT-3, 6, and 7) GluT-expressing structures. However, the fact that KO of either proximal or distal GluTs resulted in exacerbated Glu-mediated AIB responses compared to WT animals, as well as the highly compartmentalized nature of AIB synapses in regards to GluR expression pattern and synapse localization, indicates that AIB is a neuron deserving of continued examination to uncover further tiers and mechanisms for preserving signal fidelity in the glia-poor nematode neuropil.

AIB is also of great interest due to its identity as a rich club neuron, and a transducer of information from the sensory to the motor neuron strata of the nerve ring $^{14}$. As a receiver of many inputs, including other glutamatergic neurons, it is also conceivable that synaptic Glu from other circuits at varying location of the nerve ring (not restricted to just the inner and outer rim, but also potentially the cross-sections of the nerve ring structure) can spill over when the fine balance of clearance is offset when there is loss of perfusion or loss of function of any of the GluTs. A better understanding of Glu clearance at AIB offers a more complete view of the dynamic nature of Glu signaling, most notably at the intraneuronal level, and uncovers principles that can be translated to the mammalian system.

As stated previously, our GCaMP recordings of AIB neurites are preliminary and it would be worth repeated these experiments with iGluSnFR. However, attempting to image AIB iGluSnFR neurites may provide added challenges compared to our previous imaging with AVA iGluSnFR; while a map of where other neurons (which have been identified) synapse onto AVAL and AVAR has long been available ${ }^{8}$, no such guide exists for AIB. ASH synapses onto a notable protrusion on AVA neurons, providing a higher surface area to record from compared to the thin AIB neurite processes. AIB neurite GCaMP signals were already captured at the highest magnification available on the Mano lab imaging scope (100x), and measurements may have 
been limited by low signal resolution. AIB is a far more complex subject to study because of the expression of both anion- and cation-conducting Glu receptors. AVA neurons express only cation-conducting GluRs, so the nature of Glu signaling directly onto AVA is excitatory. The somatic GCaMP recordings of AIB only provides a glimpse into the net response of AIB to stimulation, but does not indicate the relative amount of Glu binding to proximal/excitatory and distal/inhibitory Glu receptors. In comparison, AIB iGluSnFR experiments could provide a more accurate reflection of Glu binding at specific neurite locations and validate whether preferential binding occurs (according to Kuramochi and Doi's hypothesis) in wild-type worms, and how GluT KO can offset relative concentration and binding location of Glu signals. Due to the challenges presented in imaging AIB, another interneuron of interest to study would be RIA, which also receives inputs from multiple sensory neurons and synapses with head motor neurons ${ }^{29}$. RIA has also been previously found to spatially encodes head movement on a subcellular scale through axonal compartmentalization ${ }^{29}$ (similar to the configuration for AIB proposed by Kuramochi and Doi) and is known to express multiple Glu receptors ${ }^{30}$, factors which make this neuron an ideal candidate for continuing our studies on how GluT KO can impact intracellular Glu clearance. 


\subsection{Materials and Methods}

Strains and maintenance

All Caenorhabditis elegans strains were cultured at $20^{\circ} \mathrm{C}$ on $\mathrm{MYOB}$ plates ${ }^{31,32}$ with Escherichia coli strain OP50 as food source. The strains used for this study are: For GCaMP6 imaging in

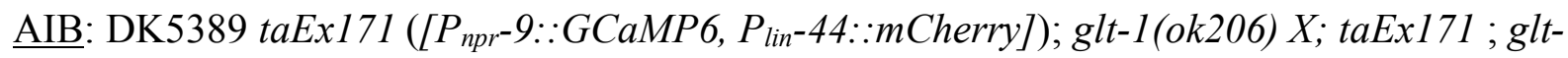
4(bz69) X, taEx171; glt-3(bz34), glt-6(tm1316), glt-7(tm1641) IV; taEx171. Details of the glt mutant strains were previously described ${ }^{33}$. The strains carrying glt mutations and AIB neurons expressing GCaMP were generated by crosses between the corresponding glt mutants and the DK5389 strain. DK5389 34 was a gift obtained from the Doi lab (National Institute of Advance Industrial Science and Technology (AIST) $)^{17}$. For specific expression of HisCl channels, glt mutant strains expressing GCaMP in AIB neurons were injected with both $P_{\text {myo- }}$ ${ }_{2}::$ HisCl1::SL2::mCherry and $P_{m y o-3}: \because$ HiSC $11:: S L 2:: m$ Cherry constructs for pharyngeal and body wall muscle expression, respectively. These constructs were generated through Gibson assembly ${ }^{35}$, as described in greater detail below.

Molecular biology

For all plasmid constructs with cDNA, total RNA was extracted from C. elegans Bristol strain N2 in TRIzol (Invitrogen) using RNeasy Mini Kit (Qiagen). RNA was used as template to create an cDNA prep using Invitrogen SuperScript ${ }^{\circledR}$ III and Oligo(dT)20 following manufacture’s protocol. All PCR fragments were amplified using NEB Q5 polymerase and were either purified via spin column purification or gel purified using NEB Monarch ${ }^{\circledR}$ DNA Gel Extraction Kit. Plasmids containing the SL2 trans-splicing signal results in a single transcript that is processed into two mRNAs and therefore two separate proteins. All plasmids were assembled via 
Gibson/HIFI assembly ${ }^{35}$ using NEB HiFi Assembly following manufacturer's protocol. Constructs were transformed in Invitrogen DH5a Competent Cells (Cat \# 18265017). Construction of $P_{m y o-2}:$ HisCl1 $\because:$ SL $2: \because$ mCherry

The 976 bp myo-2 promoter was amplified from the construct pCFJ421 [P $\left.P_{m y o-2}: \because G F P: \because H 2 B\right]$ from Erik Jorgensen (Addgene plasmid \#34876) ${ }^{36}$, using primers 5'GCCGGCCCATTTTATATCTGAGTAGTATCCTTTGCTTTAAATGTCCA and 5'GGCGCGCCTTCTGTGTCTGACGATCGAGGG. Plasmid vector construct pNP471 containing the HisCl1 sequence was PCR linearized using primers 5'-CACAGAAGGCGCGCCTCTAGAG and 5'- ATAAAATGGGCCGGCCCAGTCAG from the construct pNP471 [ $P_{\text {rig- }}$

$3: \because$ HisCl1::SL2::mCherry $]^{22}$ (removing the rig-3 promoter sequence in the process), obtained via the Bargmann lab (Rockefeller U.). The Gibson-assembled product was used to subsequently transform DH5 $\alpha$ cells.

Construction of $P_{m y o-3}: \because$ HisCl1::SL2::mCherry

The $2 \mathrm{~kb}$ myo-3 promoter was amplified from the construct pCFJ104 [P $P_{m y o-3}:: m$ Cherry::unc-54] from Erik Jorgensen (Addgene plasmid \#19328) ${ }^{37}$, using primers 5'-

CACTGACTGGGCCGGTGTGTGTGATTGCTTTTTCACAATCAGTGTTTTCAGG and 5'GGGGATCCTCTAGAGGCATTTCTAGATGGATCTAGTGGTCGT. Plasmid vector construct pNP471 22 containing the HisCl1 sequence was linearized using restriction enzymes FseI and AscI. The Gibson-assembled product was used to subsequently transform DH5 $\alpha$ cells. Construction of $P_{n p r-9::}: g l r-1:: m$ Cherry

The $2 \mathrm{~kb}$ npr-9 promoter was amplified from genomic C. elegans DNA, using primers 5'TGCACTTATACAGGCCAGAACTATTCGGGC and 5'ATGACGACATTTCCCAGGAAGTAGCTCTAAAATTACAATAAAGAC. The $4.5 \mathrm{~kb} g l r-1$ sequence 
was amplified from genomic C. elegans DNA, using primers 5'-

TTAGAGCTACTTCCTGGGAAATGTTTTCTTCGTTTTCTTTTTTG and 5’-

GAACCGCCTCCAGATCCTCCTCAGACAGCTGTGTTGTAGA. A pre-existing construct

containing the cep-1 cDNA and mCherry sequences in the KP899 vector was PCR linearized using primers 5' - TCTACAACACAGCTGTCTGAGGAGGATCTGGAGGCGGTTC and 5' -

GCCCGAATAGTTCTGGCCTGAGCTGTTTCCTGTGTGAAATTG. The Gibson-assembled product

was used to subsequently transform DH5 $\alpha$ cells.

Construction of $P_{n p r-9:: e a t-4:: m T a g B F P 2}$

The $2 \mathrm{~kb}$ npr-9 promoter was amplified from genomic C. elegans DNA, using primers 5'TGCACTTATACAGGCCAGAACTATTCGGGC and 5'-

ATGACGACATTTCCCAGGAAGTAGCTCTAAAATTACAATAAAGAC. eat-4 cDNA was amplified from genomic C. elegans DNA using primers 5'- TTCCTGGGAAATGTCGTCATGGAACGAGGC and 5'- GCTCTGACATCCACTGCTGATAATGCGGATTTTCC. A pre-existing construct containing both $g l t-3$ promoter and cDNA sequences inserted into the pCFJ150 vector ${ }^{38}\left(P_{g l t-}\right.$ 3::glt3cDNA::SL2mCherry::unc-54 3'UTR MosSCI) was PCR linearized using primers 5'GCTTAATTAAGAGCTCCGCATCGGC and 5'TTCTGGCCTGTATAAGTGCAAGTAAGATCAGTGTTTGTTTCGATATCAG (removing the $g l t-3$ promoter and cDNA sequences in the process). The Gibson-assembled product was used to subsequently transform DH5 $\alpha$ cells.

Microfluidics and imaging

Prior to imaging, adult worms were first transferred onto an unseeded MYOB holding plate. Worms were allowed to traverse the holding plate for 10 minutes, removing excess bacteria 
adhering to their bodies. For the salt stimulation experiments, solution consisting of $1 \mathrm{mM}$ $\mathrm{MgSO}_{4}, 1 \mathrm{mM} \mathrm{CaCl}_{2}$, and $5 \mathrm{mM} \mathrm{KPO}_{4}$ (salt stimulation buffer) was used ${ }^{39}$.

The Chronis \& Bargmann worm behavioral chip (Purchased from MicroKosmos) was used for GCaMP imaging experiments ${ }^{40}$. This system allows for temporal stimulation of amphid sensory neurons located on the worm nose. Worms were physically restrained in a specially fitted channel (the worm trap) designed to hold adults without the use of paralytic agents that may interfere with normal physiology. A system of liquid streams controlled under laminar flow were manipulated to present either control buffer or stimulus to the worm nose ${ }^{40}$ via a three-way electric valve (Lee Company). GCaMP transients from live nematodes trapped in the chip were recorded as previously described by Chronis \& Bargmann. To generate the stimulant solutions, $50 \mathrm{mM}$ or $1 \mathrm{mM} \mathrm{NaCl}$ was dissolved in salt stimulation buffer. For histamine-induced paralysis experiments, $1 \mathrm{M}$ histamine (VWR) was dissolved in water. The histamine solution was introduced via the buffer channel of the microfluidics system to paralyze the anterior portion of the worm. Paralysis was induced by exposing the head of the immobilized animals contained in the worm trap of the microfluidics chamber to the histamine solution for no more than 5 minutes. Our imaging system consists of a Zeiss Axiovert $200 \mathrm{M}$ motorized inverted microscope, Lumencor SOLA solid state white light source, Ludl filter wheel controller, Q Imaging EXiTM Blue camera, and ValveBank4 controller (AutoMate). Metamorph software (Molecular Devices) was used for image processing and acquisition. GCaMP transients were captured with a 63x (for GCaMP) or 100x (for iGluSnFR) objective lens at 1.67 frames/s for each experiment duration. We used $\Delta \mathrm{F} / \mathrm{F}$ to indicate change in fluorescence intensity. $\mathrm{F}$ was defined as the baseline fluorescence intensity of AIB during a period of 10 seconds. Prior to recording, worms were held 
for one minute in the microfluidics chamber in the presence of blue light to eliminate avoidance mediated through the photoreceptor protein LITE- ${ }^{41}$. Intensity measurements were restricted to AIB cell body for GCaMP imaging. This was achieved by first setting an inclusive intensity threshold to define the range of fluorescence to capture (i.e., the neuronal soma or neurite processes, which has higher intensity compared to other cells and structures), then defining a region of interest (ROI) to capture from. For GCaMP6 imaging of neuronal cell body, intensity values of light objects were defined and quantified by Metamorph's Inclusive Threshold function within the ROI for each timepoint. For neurite recordings, specific neurite regions were first located by seeking out the corresponding red and blue labels (for proximal and distal neurites, respectively. ROIs were then drawn at these locations and reporter transients were collected in response to stimulation. Reporter transients were analyzed by comparing subsequent intensity readouts to the baseline intensity, expressed as a change in percentage. The time windows (delineated by boxes outlined in green) for calculating intensity values for comparison of averages of relative stable states for each experiment was determined based on prior observations of the timing of neuronal responses, ensuring that the rise and relative stable period in signal intensity could be captured across all imaged strains. Latter time windows in Figure 29A \& D (delineated by boxes outlined in brown) were set based on when we could visualize differences in intensity values between wild type and GluT KO strains at the tail end of stimulation.

\section{Statistical analysis}

All statistical analyses was performed utilizing GraphPad Prism software. For GCaMP data, ANOVA with post hoc Bonferroni test was used for multiple group comparison of their means. Error bars denote SEM, and statistical significance is noted with * based on P values. 


\subsection{Acknowledgements}

I would like to thank Irving Estevez and Adem Idrizi for plasmid construction. I would also like to thank Sruti Patoori, Adanna Alexander, Zelda Mendelowitz, Ayesha Chowdhury, and other members from both the Li and Emerson labs for the many insightful discussions and help with the molecular biology. I would also like to express tremendous thanks to M. Katz, S. Shaham, C. Bargmann, and S. Chalasani for their extensive guidance in setting up and running the microfluidics system. I thank C. Bargmann and E. Jorgensen for the gift of plasmid constructs, and M. Doi for the gift of the AIB GCaMP6 strain. 


\subsection{References}

1.Meldrum, B. S. Glutamate as a Neurotransmitter in the Brain: Review of Physiology and Pathology. The Journal of Nutrition 130, 1007S-1015S (2000).

2. Arriza, J. L., Eliasof, S., Kavanaugh, M. P. \& Amara, S. G. Excitatory amino acid transporter 5, a retinal glutamate transporter coupled to a chloride conductance. Proceedings of the National Academy of Sciences 94, 4155-4160 (1997).

3. Tzingounis, A. V. \& Wadiche, J. I. Glutamate transporters: confining runaway excitation by shaping synaptic transmission. Nature Reviews Neuroscience 8, 935-947 (2007).

4. Suzuki, H. et al. Functional asymmetry in Caenorhabditis elegans taste neurons and its computational role in chemotaxis. Nature 454, 114-117 (2008).

5. Yu, S., Avery, L., Baude, E. \& Garbers, D. L. Guanylyl cyclase expression in specific sensory neurons: A new family of chemosensory receptors. Proceedings of the National Academy of Sciences 94, 3384-3387 (1997).

6. Pierce-Shimomura, J. T., Faumont, S., Gaston, M. R., Pearson, B. J. \& Lockery, S. R. The homeobox gene lim-6 is required for distinct chemosensory representations in C. elegans. Nature 410, 694-698 (2001).

7. Johnston, R. J., Chang, S., Etchberger, J. F., Ortiz, C. O. \& Hobert, O. MicroRNAs acting in a double-negative feedback loop to control a neuronal cell fate decision. Proceedings of the National Academy of Sciences 102, 12449-12454 (2005).

8. Altun, Z. F. et al. Wormatlas. http://www.wormatlas.org/ (2002).

9. Wakabayashi, T., Kitagawa, I. \& Shingai, R. Neurons regulating the duration of forward locomotion in Caenorhabditis elegans. Neuroscience Research 50, 103-111 (2004). 
10. Wang, L. et al. A Gustatory Neural Circuit of Caenorhabditis elegans Generates

Memory-Dependent Behaviors in $\mathrm{Na}^{+}$Chemotaxis. The Journal of Neuroscience 37, 2097-2111 (2017).

11. Kunitomo, H. et al. Concentration memory-dependent synaptic plasticity of a taste circuit regulates salt concentration chemotaxis in Caenorhabditis elegans. Nature Communications $\mathbf{4}$, (2013).

12. Jin, X., Pokala, N. \& Bargmann, C. I. Distinct Circuits for the Formation and Retrieval of an Imprinted Olfactory Memory. Cell 164, 632-643 (2016).

13. Towlson, E. K., Vertes, P. E., Ahnert, S. E., Schafer, W. R. \& Bullmore, E. T. The Rich Club of the C. elegans Neuronal Connectome. Journal of Neuroscience 33, 6380-6387 (2013).

14. Moyle, M. W. et al. Structural and developmental principles of neuropil assembly in $C$. elegans. Nature 591, 99-104 (2021).

15. Rabinowitch, I. et al. Circumventing neural damage in a C. elegans chemosensory circuit using genetically engineered synapses. Cell Systems 12, 263-271.e4 (2021).

16. Witvliet, D. et al. Connectomes across development reveal principles of brain maturation in C. elegans. http://biorxiv.org/lookup/doi/10.1101/2020.04.30.066209 (2020) doi:10.1101/2020.04.30.066209.

17. Kuramochi, M. \& Doi, M. An Excitatory/Inhibitory Switch From Asymmetric Sensory Neurons Defines Postsynaptic Tuning for a Rapid Response to $\mathrm{NaCl}$ in Caenorhabditis elegans. Frontiers in Molecular Neuroscience 11, (2019).

18. Hart, A. C., Sims, S. \& Kaplan, J. M. Synaptic code for sensory modalities revealed by $C$. elegans GLR-1 glutamate receptor. Nature 378, 82-85 (1995). 
19. Cully, D. F. et al. Cloning of an avermectin-sensitive glutamate-gated chloride channel from Caenorhabditis elegans. Nature 371, 707-711 (1994).

20. Brittin, C. A., Cook, S. J., Hall, D. H., Emmons, S. W. \& Cohen, N. Volumetric reconstruction of main Caenorhabditis elegans neuropil at two different time points. http://biorxiv.org/lookup/doi/10.1101/485771 (2018) doi:10.1101/485771.

21. Chan, J., Lee, K. K., Wong, J. C. Y., Morocho, P. \& Mano, I. Novel Strategies for Glutamate Clearance in the Glia-Deprived Synaptic Hub of C. elegans. bioRxiv (2019) doi:10.1101/645812.

22. Pokala, N., Liu, Q., Gordus, A. \& Bargmann, C. I. Inducible and titratable silencing of Caenorhabditis elegans neurons in vivo with histamine-gated chloride channels. Proceedings of the National Academy of Sciences 111, 2770-2775 (2014).

23. Aceves, J., Erlij, D. \& Martínez-Marañón, R. The mechanism of the paralysing action of tetramisole on Ascaris somatic muscle. British Journal of Pharmacology 38, 602-607 (1970).

24. Lewis, J. A., Fleming, J. T., McLafferty, S., Murphy, H. \& Wu, C. The Levamisole Receptor, a Cholinergic Receptor of the Nematode Caenorhabditis elegans. Molecular Pharmacology 31, 185-193 (1986).

25. Goodman, M. B. \& Chalfie, M. Paralysis of worms by 2,3-butanedione monoxime (BDM). Worm Breeder's Gazette 15, 9 (1998).

26. Sellin, L. C. \& McArdle, J. J. Multiple Effects of 2,3-Butanedione Monoxime. Pharmacology \& Toxicology 74, 305-313 (1994).

27. Fan, J. et al. A muscle-epidermis-glia signaling axis sustains synaptic specificity during allometric growth in C. elegans. eLife 9, (2020). 
28. Jantsch-Plunger, V. \& Fire, A. Combinatorial structure of a body muscle-specific transcriptional enhancer in Caenorhabditis elegans. Journal of Biological Chemistry 269, 27021-27028 (1994).

29. Hendricks, M., Ha, H., Maffey, N. \& Zhang, Y. Compartmentalized calcium dynamics in a C. elegans interneuron encode head movement. Nature 487, 99-103 (2012).

30. Emmons, S. W. The C. elegans Wiring Project. A database for C. elegans neuronal connectivity data. http://wormwiring.org/ (2020).

31. Brenner, S. The Genetics of Caenorhabditis elegans. Genetics 77, 71-94 (1974).

32. Church, D. L., Guan, K.-L. \& Lambie, E. J. Three genes of the MAP kinase cascade, mek-2, mpk-1/sur-1 and let-60 ras, are required for meiotic cell cycle progression in Caenorhabditis elegans. 11 (1995).

33. Mano, I., Straud, S. \& Driscoll, M. Caenorhabditis elegans Glutamate Transporters Influence Synaptic Function and Behavior at Sites Distant from the Synapse. Journal of Biological Chemistry 282, 34412-34419 (2007).

34. Shipley, F. B., Clark, C. M., Alkema, M. J. \& Leifer, A. M. Simultaneous optogenetic manipulation and calcium imaging in freely moving C. elegans. Frontiers in Neural Circuits $\mathbf{8}$, (2014).

35. Gibson, D. G. et al. Enzymatic assembly of DNA molecules up to several hundred kilobases. Nat Methods 6, 343-345 (2009).

36. Frøkjær-Jensen, C., Davis, M. W., Ailion, M. \& Jorgensen, E. M. Improved Mos1mediated transgenesis in C. elegans. Nat Methods 9, 117-118 (2012).

37. Frøkjær-Jensen, C. et al. Single-copy insertion of transgenes in Caenorhabditis elegans. Nat Genet 40, 1375-1383 (2008). 
38. Frøkjær-Jensen, C. et al. Random and targeted transgene insertion in Caenorhabditis elegans using a modified Mos1 transposon. Nat Methods 11, 529-534 (2014).

39. Oda, S., Tomioka, M. \& Iino, Y. Neuronal plasticity regulated by the insulin-like signaling pathway underlies salt chemotaxis learning in Caenorhabditis elegans. Journal of Neurophysiology 106, 301-308 (2011).

40. Chronis, N., Zimmer, M. \& Bargmann, C. I. Microfluidics for in vivo imaging of neuronal and behavioral activity in Caenorhabditis elegans. Nature Methods 4, 727-731 (2007). 41. Yemini, E. et al. NeuroPAL: A Multicolor Atlas for Whole-Brain Neuronal Identification in C. elegans. Cell 184, 272-288.e11 (2021). 


\section{Chapter 4}

\section{Conclusions, Discussions, and Further Experiments}

\section{$\underline{4.1 \text { Conclusions and Discussion }}$}

Regulation of Glu neurotransmission poses unique challenges; the lack of enzymatic degradation mechanism for curtailing excess Glu signals at the synapse, and the ubiquity of Glu across all cell types are characteristic of the major excitatory neurotransmitter in the mammalian brain. Glu synapses in the brain that utilize synaptic (wired) communication are enveloped by glial processes, which provide a physical barrier preventing spillover between adjacent circuits. However, advancements in imaging and sample preparation of the mammalian brain have revealed that extracellular space in the brain represents a much greater volume of the brain than previously expected. Coupled with the fact that some vital regions of the brain display poor glia coverage and synaptic insulation, utilizing volumetric transmission for Glu signaling, this supports the sizable role of non-glial mechanisms in Glu signal regulation. In our C. elegans model for studying Glu signaling in a glia-poor system, we have found that pharyngeal pumping and movement of the head muscle provide preside over signaling in ASH circuit synapses, in addition to preventing spillover of Glu signals from the neighboring ASE circuit. Removal of pharyngeal and head muscles movement through chemical paralysis appears to result in loss of response to stimulation, perhaps desensitizing interneuron AVA to subsequent excitatory signals (Figure 23 \& 24). We observe similar results in our somatic AIB recordings during histamineinduced paralysis (Figure 30 \& 31). While we propose that paralysis removes perfusion and 
subsequently removes a robust clearance mechanism that prevents desensitization from Glu overaccumulation, a variety of other explanations are plausible. One alternative possibility is that rather than maintaining low physiological levels of Glu at the post-synapse, pharyngeal pulsations instead facilitate accumulation of Glu. In this model, paralysis may actually eliminate AVA/AIB responses to stimulation by preventing synaptic Glu to accumulate high enough levels to illicit a sizable neuronal response. While we currently have not ascertained the effect of paralysis on synaptic Glu levels in the nematode system, we strongly believe that loss of function of distal GluT glt-3 results in elevated Glu concentrations at nerve ring synapses. Previous findings from Feldmann et al., 2019 indicate that $C$. elegans containing both glt-3 $\mathrm{KO}$ and a constitutively active form of the alpha subunit of the G protein Gs $\left(\mathrm{G} \alpha \mathrm{s}^{*}\right)$ display higher levels of neurodegeneration of $g l r-1$-expressing neurons via excitotoxic necrosis ${ }^{1-3}$. Intriguingly, animals with loss of function of other GluTs did not have as high a level of neurodegeneration when paired with $\mathrm{G} \alpha \mathrm{s}^{*}$, or animals without any GluT mutations that expressed $\mathrm{G} \alpha \mathrm{s}^{* 3}$. This may indicate that $g l t-3 \mathrm{KO}$ elevates Glu to high enough levels to strongly enhance neurodegeneration, and thus may play a larger role in synaptic Glu clearance than the other GluTs. Whether Glu concentration is elevated specifically in nerve ring circuits or throughout the interstitial fluid of the nematode is unknown, and is a question of interest to pursue.

We also sought to answer whether in addition to differences in expression pattern, if different classes of GluTs may also have functional differences in clearance activity due to respective differences in key Glu-interacting motifs. Our promoter swap experiments provide a preliminary glimpse into how different GluTs affect clearance when expressed proximally or distally from the synapses of specific behavioral circuits (Figures $17 \&$ 18). However, our focus was primarily on the physiological activity of a single interneuron of a behavioral circuit, and not 
on the network of connections and complex interactions with other circuits in the synaptic hub. Further experiments are needed to determine whether functional differences exist between the two classes of GluTs. There is also the question of whether GluTs are essential for survival; behavioral assays have shown that while Glu-mediated behaviors are impacted in GluT KO mutants (Figures 6 \& 10), our GluT KO strains do not appear to display any developmental delays or other apparent differences compared to wild type animals. GluT KO mutants are able to survive until adulthood (though lifespan assays will need to be performed to measure whether GluT KOs have any effect on longevity). While it would be of interest to see the overall effect of KO for all C. elegans transporters, all previous efforts to generate a proximal GluT double KO strain for $g l t-1$ and $g l t-4$ have failed. This could indicate that absence of activity of both proximal GluTs is lethal to the nematode. RNA interference (RNAi) for $g l t-1$ and $g l t-4$ can be used instead to suppress expression. RNAi for both proximal GluTs can also be introduced to $g l t-3,6,7$ mutants to generate loss of function mutants for all GluTs. The resulting effects on signaling and Glu accumulation can then be measured using GCaMP and iGluSnFR reporters at the physiological level in neurons and other structures, in addition to gauging any impacts on life span and behavior through respective assays. Generating loss of function mutants for all nematode GluTs could answer the question of whether GluTs are at all vital in clearance of Glu synapses, or whether they share a more minor role in clearance while perfusion and mechanical agitation of fluids from the nerve ring is sufficient to maintain functional Glu circuits. It should also be noted that other modes of signaling may compensate for loss of function of GluTs in Glumediated behaviors, one of the most prominent being electrical signaling via gap junctions. Physically linking adjacent neurons through channels, electrical signaling between neurons occurs independently of perfusion. A major gap junction exists between rich club interneurons 
AVA and AIB, and both are known to mediate responses to quinine (detected by ASH) ${ }^{4}$ and odors such as isoamyl alcohol (detected by AWC) 5 . Intriguingly, our experiments had shown that ectopic excitatory responses in AVA to a $1 \mathrm{mM} \mathrm{NaCl}$ upstep occurred in proximal GluT mutants (Figure 11A). Considering the gap junction between AVA and AIB, this was unexpected because ASEL stimulation with $\mathrm{NaCl}$ concentration upstep has an inhibitory effect on AIB neurons, would have been predicted to inhibit AVA. Additionally, a $1 \mathrm{mM} \mathrm{NaCl}$ downstep stimulating ASER activates AIB and should also trigger an excitatory responses in AVA, but in Figure 13A we see that this is not the case. It is entirely possible that other neuronal signals and differences in wiring contribute to these differences between AVA and AIB responses. While our data so far with AVA responses to salt stimulation has not been a thorough investigation on AVA-AIB synapses, gap junctions are still a prominent mode of signaling predicted to continue activating neurons and partially compensating for aberrant signaling under GluT KO conditions.

A GluT which has not been investigated in our promoter swap experiment is GLT-4; expressed pre-synaptically in some neurons (though the identities of only a few of them have been verified), we believe it may have significant chloride conducting activity (much like the role of hEAAT5 in the pre-synaptic bipolar cells of the mammalian retina $)^{6,7}$. Retinal bipolar cells constitutively release Glu in the absence of light ${ }^{8,9}$, and uncoupled $\mathrm{Cl}^{-}$conductance through hEAAT5 when the transporter channel opens during Glu transport creates a negative feedback loop that reduces excitatory Glu transmission ${ }^{8,10,11}$. Neuronally-expressed GLT-4 may function in the same way to curtail Glu transmission: a constitutively active sensory neuron, $\mathrm{AWC}^{\mathrm{ON}}$, releases Glu in absence of odorants (such as butanone) ${ }^{12}$, and expression of $g l t-4$ on the presynapse or in adjacent neurons may also negatively regulate $\mathrm{AWC}^{\mathrm{ON}}$ activity. Additionally, while loss of GluT function may result in hyperexcitation and Glu spillover, the negative feedback 
mechanism of $\mathrm{Cl}^{-}$conductance at pre-synapses is removed as well. This could result in deregulation of other modes of excitatory neuronal signaling. For example, dopamine-dependent swimming behavior is known to be modulated by Glu signaling and glial-expressed glt-1, glt-4, and $g l t-3^{13}$, and gap junctions found widely throughout the nerve ring also allow serve as a separate mode of neuronal activation (though the strength of some gap junctions are actually modulated by stimulation of Glu synapses $)^{14,15}$, though the degree it would impact other neuronal signaling pathways is not yet known. It should also be noted that the sequences of the second transmembrane domain (TM2) of the $C$. elegans GluTs had very low sequence homology to hEAAT5 and hEAAT1 (Figure 39). In addition to influencing Glu transport, TM2 contains residues that have impacted $\mathrm{Cl}^{-}$conductance when substituted (most significantly, residue $\mathrm{S} 103$ in hEAAT1, boxed in red and marked with asterisk in Figure 39) ${ }^{16}$. Since S103 is preserved in hEAAT5 and the $C$. elegans GluTs, the possibility that all $C$. elegans GluTs may have significant $\mathrm{Cl}^{-}$conducting capabilities cannot be discounted. Further exploration of TM2 and other domains known to be involved in $\mathrm{Cl}^{-}$conductance (including residues from HP1, TM5, and TM7) ${ }^{17}$ in $C$. elegans GluTs will be needed to determine these roles.

\begin{tabular}{|c|c|}
\hline hEAAT1 & VKYFSFPGELLMRMLQMLVLPLIISSLVTGMAALDSKASGKMGMRAVVYYMTTTIIAVV \\
\hline hEAAT5 & IMYISFPGELLMHMLKMMILPLIMSSLISGLAQLDARQSGKLGSLAVTYYMFTTAVAVV \\
\hline CeGLT3 & ISYFQFPGELLMRMLKMMILPLVVSSLMSGLASLDAKTSSRLGVLTVAYYLWTTFMAVI \\
\hline CeGLT4 & LQLINFPGEIFMQVLKMMILPLIFSSLISALAQMDAKESGQMGASTVLYYLSTAVLATI \\
\hline CeGLT1 & LSYLRFPGDLFVQMLKMLILPMIMSSI ITSLASLDSGTAGRLGMVSMIYYTLTTFFAV \\
\hline CeGLT6 & FPGEIFMQVLKLMVLPLIFSSLISSLGQMDASRSGKMSFIAIAYYLTTVIIA \\
\hline CeGLT7 & FPGEIFMQIVEMMILPLIMSSVISALAQVRARDARRIGIVTIIYYMITTFLA \\
\hline
\end{tabular}

Figure 39. Alignment of $\boldsymbol{C}$. elegans GluT domain TM2 with hEAAT1. C. elegans GluTs and hEAAT5 are ranked in descending order of sequence identity to hEAAT1. Residue S103 in hEAAT1 and corresponding residues are boxed in red and marked with asterisk.

We believe that a combination of perfusion and differentially localized, functionally distinct GluTs in the nematode system are mechanisms of Glu regulation that may also be conserved in the mammalian CNS. Further investigating perfusion and clearance mechanisms 
can lead to increased efficiency of targeted delivery for therapeutic interventions. For example, with a better understanding patterns of vasomotion and brain oscillation, we can better chart areas that circumvent the blood brain barrier and localize treatments to specific brain compartments to treat tumors. Devising ways to manipulate brain pulsations of interstitial fluid and heart rate to promote clearance of solutes and aggregates (such as amyloid $\beta$ ) in patients also has the potential to mitigate the onset of Alzheimer's disease and other neuropathies.

\subsection{Future Experiments}

\subsubsection{Analyzing GluT properties through heterologous expression in Xenopus oocytes.}

While our promoter swapping experiments in C. elegans revealed that some GluTs may have functional differences, microinjection of the GluT constructs to generate transgenic animals present several caveats: this technique results in over-expression and introduces variability in the number of plasmid copies inherited by transformant animals and their progeny. One solution would be to generate single-copy integration animals for the GluT rescue constructs through MosSCI ${ }^{18-20}$. However, a more direct and established method of studying electrogenic GluT transport and validating differences in GluT properties is to generate transgenic Xenopus oocytes and utilize a two-electrode voltage clamp electrophysiology system. We can gauge selectivity for Glu by generating dose-response curves, and transporter capacity can be compared by measuring the currents coupled and uncoupled with Glu transport. Specific motifs and residues (for example, the highly-conserved 'NMDGT' motif can be altered through Gibson assembly or sitetargeted mutagenesis. We can also measure $\mathrm{Na}^{+}$and $\mathrm{Cl}^{-}$conductance of the altered transporters 
by changing the concentration of the respective ions in the recording solution and compare the generated I/V curves.

Our predictive modeling in PyMOL ${ }^{21}$ of C. elegans GluTs built around the structure of human EAAT1 can provide insights into how sequence differences may affect ligand affinity and resulting kinetics of transport. As expected from the high degree of conservation between GluTs, the structures of our nematode GluTs models and Gouaux lab's model of Glt ${ }_{\mathrm{ph}}$ (GluT in bacteria Pyrococcus horikoshii) were largely similar (Figure 40) ${ }^{22}$. We identified sites of interest by superimposing models of nematode GluTs and selecting binding residues with unique identities or orientation, and supplemented my search with residues identified from previous literature ${ }^{22,23}$. A major target for our investigation is the highly conserved 'NMDGT' motif. In both Glt $\mathrm{ph}_{\mathrm{ph}}$ and human EAAT1, this motif has been shown to be involved in $\mathrm{Na}^{+} / \mathrm{K}^{+}$binding ${ }^{24}$. Our models have revealed that compared to the other GluTs, distal GluT GLT-3 is predicted to have a prominent difference in binding location of the second $\mathrm{Na}^{+}$ion (Figure 40B), which can be attributed to the existence of a Threonine (T342) residue in place of the Asparagine $(\mathrm{N})$ in the 'NMDGT' motif (while GLT-6 has an N (N354) in place of a T, though this does not appear to perturb localization of $\mathrm{Na}^{+}$ions in our model, Figure 41D). This motif is conserved in proximal GluTs GLT-1 (A) and GLT-4 (C). Since transport of Glu is $\mathrm{Na}^{+}$-dependent, decrease in affinity of $\mathrm{Na}^{+}$ binding can affect Glu transport and may impact the overall kinetics of transport. We predict that this residue difference observed in GLT-3 may translate into decreased affinity for the second $\mathrm{Na}^{+}$ion due to the notable distance of this ion from other residues in the binding pocket when compared with its positioning in other GLTs. Our modeling has also revealed differences in the shape of the binding pocket that affects orientation of Aspartate (Asp) ligand. Both GLT $_{\mathrm{ph}}$ and human EAAT1 have been shown to bind Asp, due to its structural similarity to Glu. Although 
$\mathrm{GLT}_{\mathrm{ph}}$ solely binds Asp and not Glu, EAAT1 has a stronger Glu preference ${ }^{23,24}$. Importantly, Asp/Glu affinity is dependent on $\mathrm{Na}^{+}$concentration; $\mathrm{Na}^{+}$binding induces a conformational change in the binding domain to become more conducive to binding Asp/Glu ${ }^{24}$. The differences in the shape of the binding pocket appear to arise from the Methionine residue (Figure 42), which are angled away from the Asp ligand in distal GluTs (Figure 42B, D) compared to proximal GluTS (Figure 42A, C). These differences in tilt may be one of many determinants of transporter affinities for Asp (and ultimately Glu). Lastly, it would also be of interest to investigate whether nematode GluT subunits contain binding sites for a third $\mathrm{Na}^{+}$ion. Human EAAT1 monomers are known to have three binding sites for $\mathrm{Na}^{+}$, and based on calculations from zero flux equations of Glu transport (with the co-transport of $3 \mathrm{Na}^{+}, 1 \mathrm{H}^{+}$, and antiport of $1 \mathrm{~K}^{+}$ ion), display a more powerful driving force for transport of Glu compared to $\mathrm{Glt}_{\mathrm{ph}}$, which cotransports $2 \mathrm{Na}^{+}$ions for each molecule of $\mathrm{Glu}^{9,24}$. Due to the high sequence and structural similarity across all GluTs, it is unknown whether C. elegans GluTs co-transport two or three $\mathrm{Na}^{+}$ions, though our modeling so far have predicted only two sites for each GluT subunit. These are some of the many substrate binding residues for targeted mutagenesis and Xenopus oocyte electrophysiology experiments in our ongoing investigation of ligand affinity and transport capacity of C. elegans GluTs. 


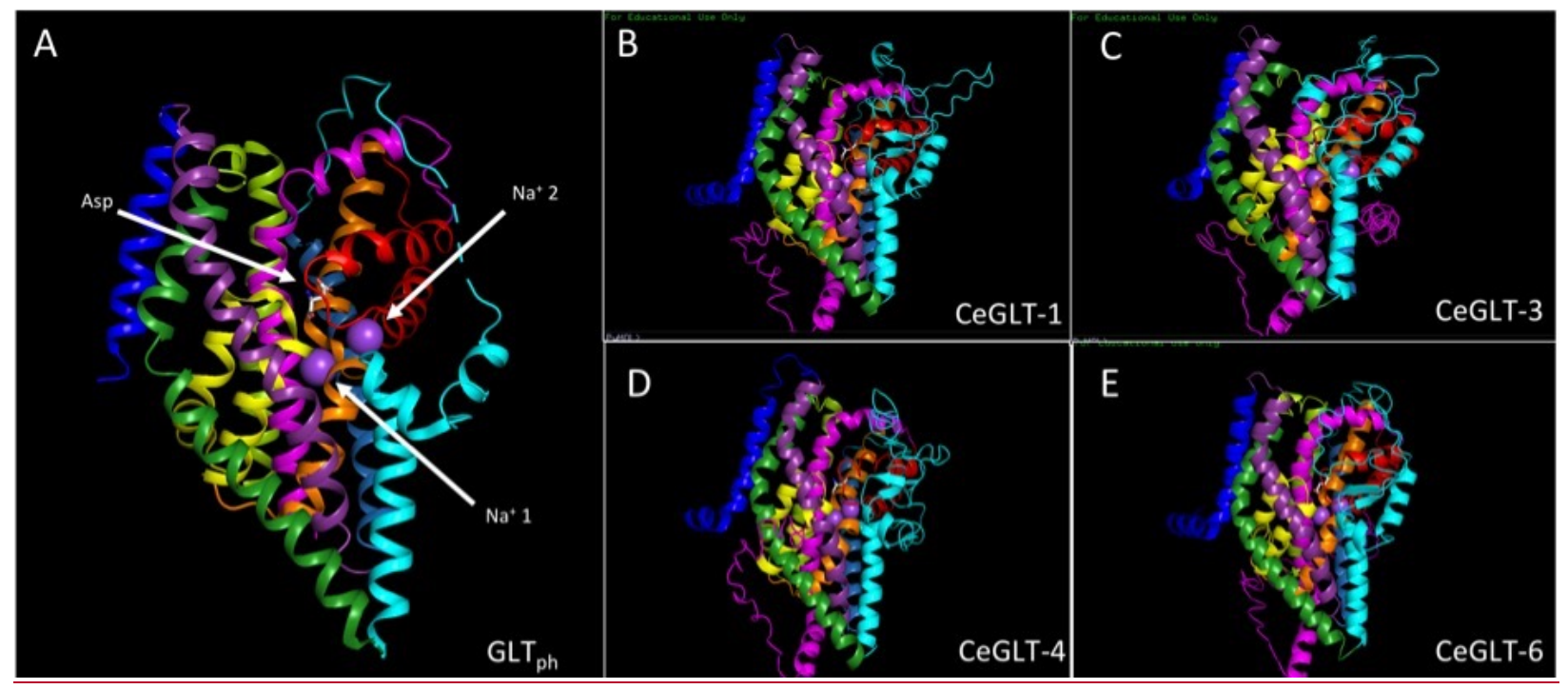

Figure 40. Predictive modeling of $C$. elegans GluTs reveals high structural similarities with bacterial Glt ph. White arrows indicate the position of substrates bound to $\mathrm{GLT}_{\mathrm{ph}}$. Core binding domains are colored as follows: HP1 (yellow), TM7 (orange), HP2 (red), TM8 (magenta). For each panel, the following structures are depicted: Glt ph (A), C. elegans GLT-1 (B), C. elegans GLT-3 (C), C. elegans GLT-4 (D), and C. elegans GLT-6 (E).

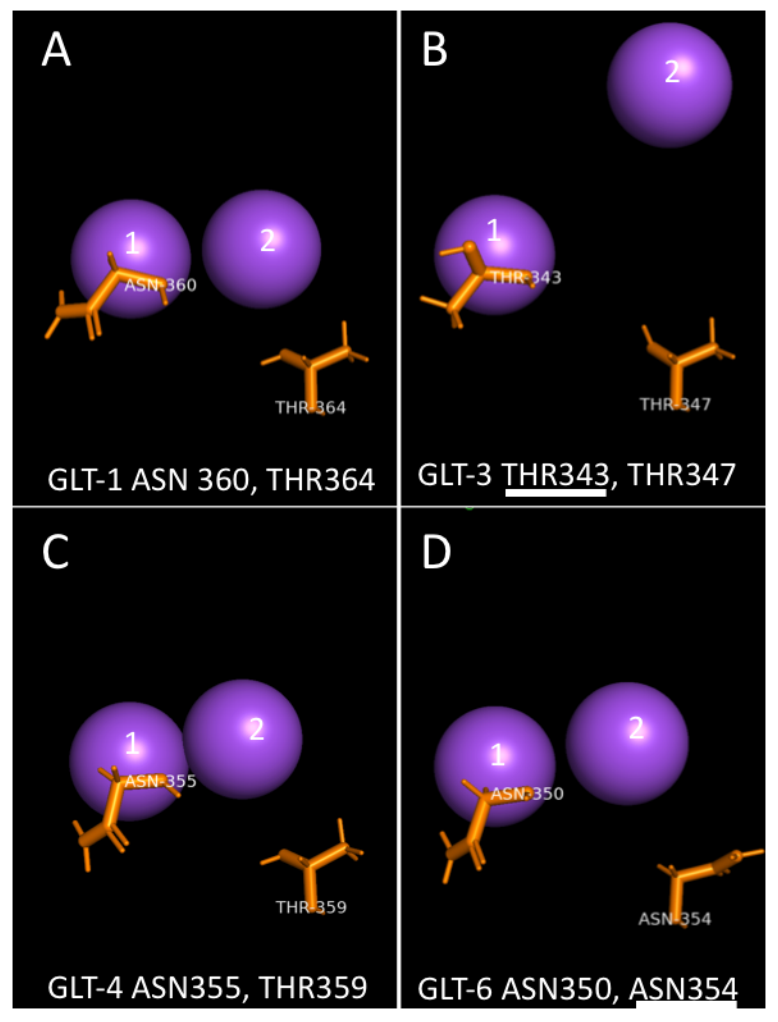

Figure 41. Differences in orientation of conserved residues can potentially affect $\mathrm{Na}^{+}$ion affinity. Side chains are shown for residues interacting with $\mathrm{Na}^{+}$ions (in purple). Conserved Asparagine and Threonine residues of the 'NMDGT' motif (depicted in orange), with the exceptions of distal GluTs GLT-3 (B), where a Threonine exists in place of the Asparagine; and GLT-6 (D), where and Asparagine exists in place of the Threonine. Panels indicate the following: (A) GLT-1; (B) GLT-3; (C) GLT-4; and (D) GLT-6. 


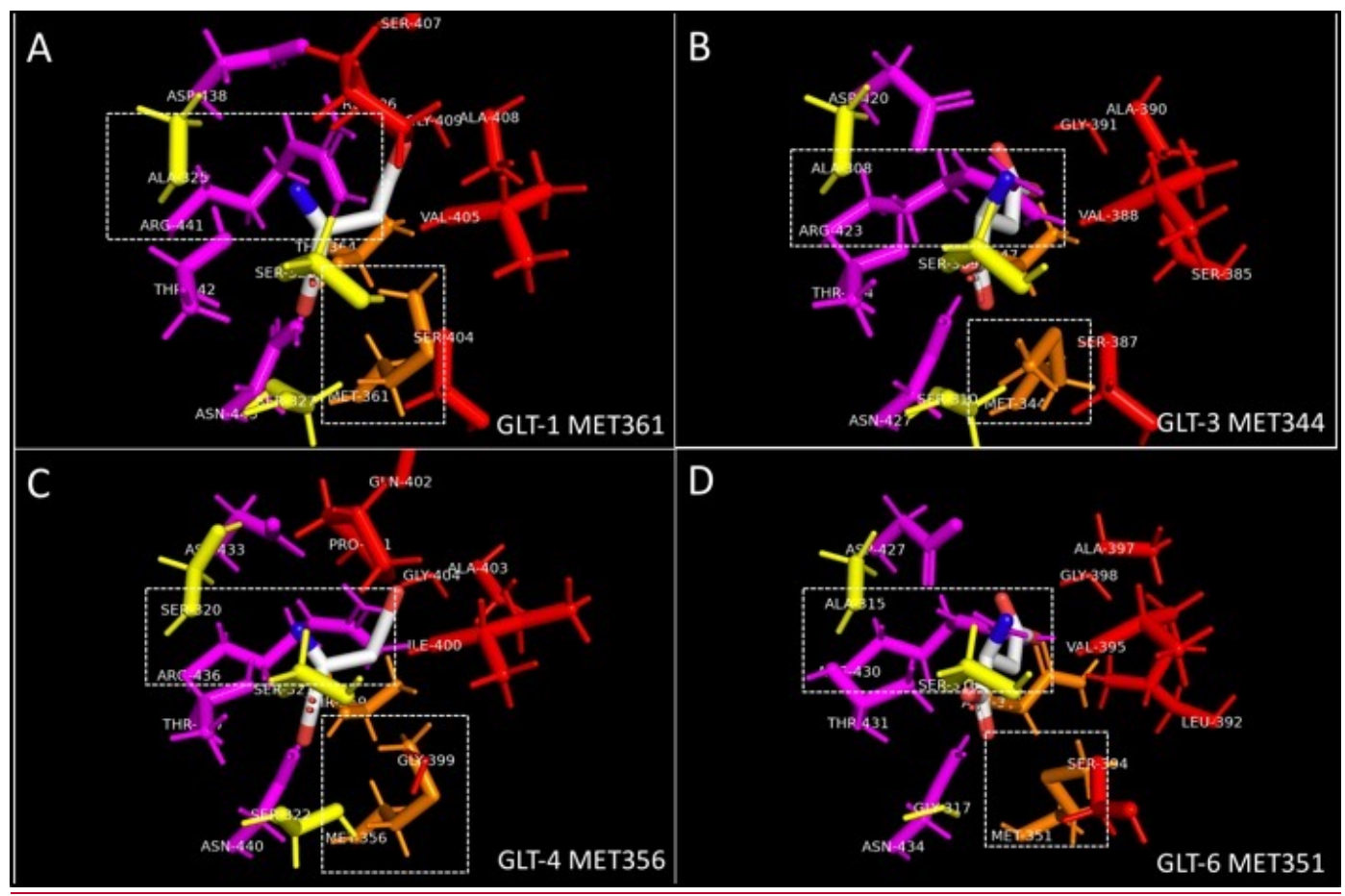

Figure 42. Differences in orientation of conserved residues can potentially affect ligand affinity. Side chains are shown for residues interacting with the Aspartate ligand (in white). The side chains of conserved the Methionine and Arginine of interest are indicated in white, dashed boxes for C. elegans A) GLT-1; B) GLT-3; C) GLT-4; and D) GLT-6. Core binding domains are colored as follows: HP1 (yellow), TM7 (orange), HP2 (red), TM8 (magenta).

\subsubsection{Determining how AIB neurons distinguishes between excitatory and inhibitory}

\section{signals when Glu serves as the neurotransmitter for both responses.}

Kuramochi and Doi (2019) found that synapse formation of the ASE neurons at regions with differentially localized GluRs determine whether Glu acts as an excitatory or inhibitory neurotransmitter on interneuron $\mathrm{AIB}^{25}$. Excitatory Glu responses arise from Glu binding to excitatory GluRs (like $g l r-1, g l r-5, m g l-1 / 2)^{4,25}$ on proximal AIB neurites, whereas the inhibitory response arises from Glu binding to inhibitory GluCls (like $g l c-3$ and $a v r-14)^{25,26}$ localized on distal AIB neurites. While informative, our $\mathrm{Ca}^{2+}$ recordings of the AIB soma only show the net result of Glu binding to excitatory and inhibitory GluRs, and resolution of recordings at neuronal processes appeared to be too poor to discern significant differences in synaptic Glu accumulation in GluT KO mutants. To dissect the roles of different GluTs on synaptic Glu clearance of AIB, 
synaptic Glu reporter iGluSnFR ${ }^{27}$ can be used to better measure and compare synaptic Glu accumulation at specific neurite regions in response to changes in $\mathrm{NaCl}$ concentration. As with the neurite recordings from AIB GCaMP6 strains, $P_{n p r-9::}: g l r-1$ - $m$ Cherry and $P_{n p r-9::}: e a t-4 c D N A$ $m T a g B F P 2$ expressed in these animals can be used to distinguish the proximal/postsynaptic and distal/presynaptic regions, respectively (Figure 43). An AIB-specific iGluSnFR construct, $\left[P_{n p r}\right.$ 9::iGluSnFR], has already been constructed and is ready for injection into GluT KO animals for the creation of new strains. Based on the AIB imaging data I have collected so far, I expect to see stronger iGluSnFR signals at AIB neurites in GluT KO animals compared to WT, due to excess Glu on the post-synaptic GluRs of GluT KO animals.

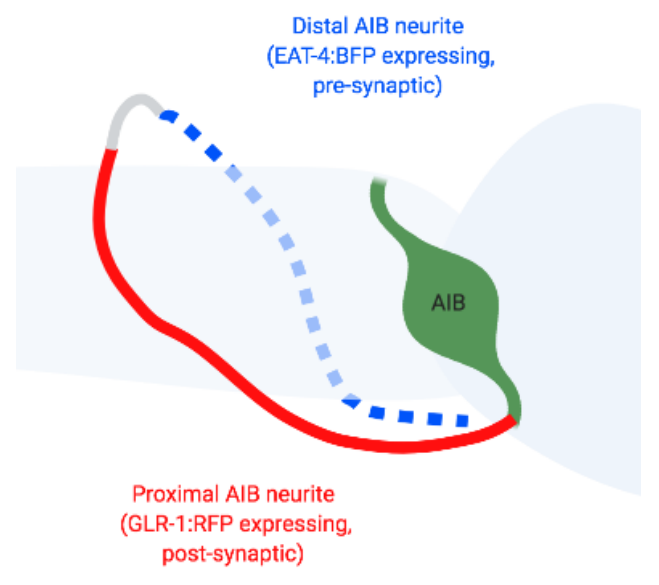

Figure 43. Schematic for distinguishing between proximal and distal neurite regions for AIBspecific iGluSnFR recording. In strains expressing AIB iGluSnFR, constructs will also be introduced to label the proximal/post-synaptic region with GLR$1:$ mCherry (red fluorescence) and the distal/presynaptic region with EAT-4:mTagBFP (blue fluorescence)

\subsubsection{Tracking diffusion and localization of Glu released from different points of origin.}

The ASE neurons are the source of Glu signals that bind directly to distinct regions of AIB neurites, and are preferentially localized based on the nature of the change in salt concentration. Since Kuramochi and Doi (2019) have data showing that sensory ASEL and ASER send Glu signals to the AIB interneurons to regulate chemotaxis, an experiment that will provide added dimension to our spatiotemporal analysis of: a) where Glu is pre-synaptically released, using ASE-specific expression of a reporter of synaptic Glu release, pHluorin $^{12}$ (driven under Pgcy-5 
and $P g c y-7$ for ASER and ASEL, respectively ${ }^{28}$ ), and; b) the timing and localization of Glu signals on the AIB neurite (indicated as intensity changes in post-synaptic iGluSnFr signals). Based on the previous salt stimulation experiments, I expect validation for part of Kuramochi and Doi's (2019) imaging findings ${ }^{25}$; our 3D neuron reconstruction (Figure 28) implies that the ASE neurons makes contact with the AIB neurons on the proximal/post-synaptic, but not distal/pre-synaptic, neurite regions. While we are confident that iGluSnFR signals will increase on the proximal/post-synaptic neurite of AIB after excitatory ASER stimulation (followed by an increase in ASER pHluorin signals as Glu is released), this experiment can answer whether ASEL excitation (and subsequent Glu release and pHluorin signal increase) during inhibitory signaling will result in a corresponding increase in iGluSnFR signals on AIB distal/pre-synaptic neurites.

We can also perform multiple experiments with iGluSnFR to visualize the diffusion and localization of Glu. To determine whether paralysis of head and pharyngeal muscles promotes accumulation of synaptic Glu to high enough concentrations to result in hyperexcitation of AVA and other interneurons, we can express iGluSnFR under the promoter of different structures. Structures of interest include the pharynx, body wall muscle, and canal cells (using promoters for myo-2, myo-3, and glt-3, respectively, which we have already cloned into constructs) to determine whether stimulation of Glu-releasing neurons and their downstream targets are able to increase Glu levels proximal and distal structures, in addition to revealing the relative timepoints of when Glu reaches these locations. iGluSnFR measurements at canal cells would be especially informative in determining whether synaptic Glu released from stimulation of ASH, ASE, or other sources actually reach the canal cells for clearance by distal GluTs. Additionally, comparing iGluSnFR signals of WT and GluT KO strains can also verify whether loss of GluT 
activity elevates local Glu levels at specific synapses. This is of particular interest for distal GluT mutants, which have shown aberrant responses at the neuronal and behavioral level. iGluSnFR signals can also be compared during paralyzed and un-paralyzed conditions to answer whether paralysis inhibits Glu clearance or promotes accumulation.

\subsubsection{Investigating where GLT-4 acts pre-synaptically as a chloride-conducting GluT to negatively regulate Glu release.}

While previous promoter-fusion experiments with GFP were successful and characterizing expression pattern of the different GluTs in C. elegans, signals were too weak to properly determine the identity of the neurons where GLT-4 is localized and expressed ${ }^{29}$. Using an extremely bright superfolder GFP reporter construct ${ }^{30}, P g l t-4:: s f G F P$, we can determine the identities of GLT-4 expressing neurons by microinjecting the construct into NeuroPAL $C$. elegans, worms that express a multicolor transgene for nervous-system-wide neuronal identification in C. elegans ${ }^{31}$. We expect to find pre-synaptic neurons expressing GLT-4, which we predict to act as a chloride conductor (much like the role of hEAAT5 in the mammalian retina) which negatively regulates excitatory release via uncoupled chloride ion conductance into the pre-synaptic neuron during Glu transport. After identifying GLT-4 - expressing neurons, we can generate strains expressing pHluorin reporter under cell-specific promoters to determine whether GLT-4 can curtail synaptic release by acting as a chloride channel. RIA has already been identified as a GLT-4 expressing neuron ${ }^{32}$, and is an ideal target since it is known to synapse directly with $\mathrm{AIB}^{33}$. Other targets identified through previous RNA-Seq studies include interneuron RIG and $\mathrm{AWC}{ }^{\mathrm{ON}}$ sensory neurons ${ }^{12,32,34,35}$. We expect RIA and other neurons identified from the reporter fusion to have lower pHluorin signals upon synaptic Glu release 
from ASEL and/or ASER (based on location of the GLT-4 neuron, there may be differential clearance activity), indicative of the chloride conductance that accompanies Glu transport.

\section{$\underline{\text { 4.3 Materials and Methods }}$}

Sequences for Gltph, human EAAT1, and C. elegans GluT sequences were obtained from NCBI database and aligned using NCBI BLAST ${ }^{36,37}$. For protein modeling, DNA sequences for the aforementioned proteins were submitted to I-TASSER, and predicted structures of each of the $C$. elegans GluTs were constructed separately based on known human EAAT1 or Gltph structures, though the resulting models and predicted substrate binding sites were highly similar between the two conditions ${ }^{38-40}$. The resulting structures were then visualized and labeled using PyMOL software $^{21}$. 


\subsection{Appendix}

4.4.1. Members of the mammalian Excitatory Amino Acid Transporter (EAAT) family.

\begin{tabular}{|c|c|c|c|c|c|}
\hline Standard name & Protein name & $\begin{array}{l}\text { Predominant } \\
\text { substrates }\end{array}$ & Tissue distribution & $\begin{array}{l}\text { Steady-state } \boldsymbol{K m} \text {, } \\
\boldsymbol{\mu} \mathrm{M}\end{array}$ & $\begin{array}{l}\text { Pre-Steady-State } \\
\text { Affinity, } \mu \mathrm{M}\end{array}$ \\
\hline SLC1A3 & EAAT1, GLAST & D/L-Asp and L-Glu & $\begin{array}{l}\text { Astrocytes } \\
\text { (Cerebellum) }\end{array}$ & $7-20$ & 20 \\
\hline SLC1A2 & EAAT2, GLT-1 & D/L-Asp and L-Glu & $\begin{array}{l}\text { Astrocytes } \\
\text { (Cerebrum) }\end{array}$ & $12-18$ & 140 \\
\hline SLC1A1 & EAAT3, EAAC1 & D/L-Asp and L-Glu & $\begin{array}{l}\text { Neurons } \\
\text { (Postsynaptic, } \\
\text { Cerebrum) }\end{array}$ & $8-28$ & 20 \\
\hline SLC1A6 & EAAT4 & D/L-Asp and L-Glu & $\begin{array}{l}\text { Neurons } \\
\text { (Postsynaptic, } \\
\text { Cerebellum) }\end{array}$ & $0.6-2.5$ & 5 \\
\hline SLC1A7 & EAAT5 & D/L-Asp and L-Glu & $\begin{array}{l}\text { Neurons } \\
\text { (Presynaptic, Retina) }\end{array}$ & $61-64$ & \\
\hline
\end{tabular}




\subsubsection{Strains used in behavioral assays and imaging experiments}

Unless indicated otherwise, strains were generated by crossing. Strains generated by microinjection have key features of each construct indicated in the description.

\begin{tabular}{|c|c|c|}
\hline Strain & Description & Source \\
\hline Bristol N2 & Wild type strain & $\begin{array}{l}\text { Caenorhabditis elegans Genome } \\
\text { Center (CGC) }{ }^{31}\end{array}$ \\
\hline $\begin{array}{l}\text { VM1268: } n m r-1(a k 4) I I ; g l r-2(a k 10), g l r- \\
\text { l(ky176) III }\end{array}$ & $\begin{array}{l}\text { Loss of function in } \\
\text { ionotropic Glutamate } \\
\text { receptors }\end{array}$ & Andres V. Maricq \\
\hline $\begin{array}{l}\text { QW625: lin-15; zfIs } 42\left[P_{\text {rig- }}\right. \\
3 \because: G C a M P 3:: S L 2:: m \text { Cherry; lin-15(+)] }\end{array}$ & $\begin{array}{l}\text { GCaMP } 3 \text { expressed in AVA } \\
\text { interneurons }\end{array}$ & Mark Alkema ${ }^{32}$ \\
\hline ZB1113: glt-1(ok206) X & Loss of function in GLT-1 & Itzhak Mano $^{24}$ \\
\hline ZB1098: glt-4(bz69) X & Loss of function in GLT-4 & Itzhak Mano $^{24}$ \\
\hline ZB1096: glt-3(bz34) IV & Loss of function in GLT-3 & Itzhak Mano $^{24}$ \\
\hline $\begin{array}{l}\text { IMN16: glt-3(bz34), glt- 6(tm1316), } \\
\text { glt-7(tm1641) IV }\end{array}$ & $\begin{array}{l}\text { Loss of function in GLT-3, } \\
\text { GLT-6, and GLT-7 }\end{array}$ & Itzhak Mano \\
\hline IMN18: glt-1(ok206) X; zfIs42 & $\begin{array}{l}\text { Loss of function in GLT-1, } \\
\text { expressing GCaMP3 in } \\
\text { AVA interneurons }\end{array}$ & Itzhak Mano \\
\hline $\begin{array}{l}\text { IMN19: glt-3(bz34), glt-6(tm1316), glt- } \\
\text { 7(tm1641) IV; zfIs42 }\end{array}$ & $\begin{array}{l}\text { Loss of function in GLT-3, } \\
\text { GLT-6, and GLT-7, } \\
\text { expressing GCaMP3 in } \\
\text { AVA interneurons }\end{array}$ & Itzhak Mano \\
\hline IMN20: $g l t-4(b z 69) X ; z f I s 42$ & $\begin{array}{l}\text { Loss of function in GLT-4, } \\
\text { expressing GCaMP3 in } \\
\text { AVA interneurons }\end{array}$ & Itzhak Mano \\
\hline $\begin{array}{l}\text { CX10979: kyEx2865[P }{ }_{\text {sra- }}: \because G C a M P 3 \text {; } \\
\text { ofm- 1::gfp] }\end{array}$ & $\begin{array}{l}\text { GCaMP3 expressed in ASH } \\
\text { sensory neuron }\end{array}$ & Dirk R. Albrecht \\
\hline $\begin{array}{l}\text { CX14652: kyEx4787 [P rig-3::iGluSnFR, } \\
\text { unc-122::dsRed] }\end{array}$ & $\begin{array}{l}\text { iGluSnFR expressed in } \\
\text { AVA interneuron }\end{array}$ & Cornelia I. Bargmann ${ }^{34}$ \\
\hline IMN50: ok206(glt-1) X; kyEx4787 & $\begin{array}{l}\text { Loss of function in GLT-1, } \\
\text { expressing iGluSnFR in } \\
\text { AVA interneurons }\end{array}$ & Itzhak Mano \\
\hline $\begin{array}{l}\text { IMN51: glt-3(bz34), glt-6 (tm1316), glt-7 } \\
\text { (tm1641) IV; kyEx4787 }\end{array}$ & $\begin{array}{l}\text { Loss of function in GLT-1, } \\
\text { GLT-3, and GLT-7, } \\
\text { expressing iGluSnFR in } \\
\text { AVA interneurons }\end{array}$ & Itzhak Mano \\
\hline IMN52: eat-4 (ky5) III ; zfIS42 & $\begin{array}{l}\text { Loss of function in EAT-4, } \\
\text { expressing GCaMP3 in } \\
\text { AVA interneurons }\end{array}$ & Itzhak Mano \\
\hline $\begin{array}{l}\text { IMN53: glt-1 (ok206) X ; eat-4 (ky5) III ; } \\
\text { zfIS42 }\end{array}$ & $\begin{array}{l}\text { Loss of function in GLT-1 } \\
\text { and EAT-4, expressing } \\
\text { GCaMP3 in AVA } \\
\text { interneurons }\end{array}$ & Itzhak Mano \\
\hline $\begin{array}{l}\text { IMN54: glt-3(bz34), glt-6(tm 1316), glt- } \\
\text { 7(tm1641) IV ; eat-4 (ky5) III ; zfIS42 }\end{array}$ & $\begin{array}{l}\text { Loss of function in GLT-3, } \\
\text { GLT-6, GLT-7 and EAT-4, }\end{array}$ & Itzhak Mano \\
\hline
\end{tabular}




\begin{tabular}{|c|c|c|}
\hline & $\begin{array}{l}\text { expressing GCaMP3 in } \\
\text { AVA interneurons }\end{array}$ & \\
\hline $\begin{array}{l}\text { TQ2905: xuEx929 [P } P_{\text {sra-6 } 6:: e a t-} \\
\text { 4(cDNA)::SL2::yfp1+P }+P_{\text {npr-9: }:: d s r e d+P u n c-} \\
\text { 122::rfp]; eat-4(ky5) III }\end{array}$ & $\begin{array}{l}\text { Loss of function in EAT-4, } \\
\text { expressing functional EAT- } \\
4 \text { cDNA specifically in ASH } \\
\text { neurons }\end{array}$ & Shawn $\mathrm{Xu}^{35}$ \\
\hline $\begin{array}{l}\text { IMN56: xuEx929; glt-1 (ok206) X ; eat-4 } \\
\text { (ky5) III ; zfIS42 }\end{array}$ & $\begin{array}{l}\text { Loss of function in GLT-1 } \\
\text { and EAT-4, expressing } \\
\text { functional EAT-4 cDNA } \\
\text { specifically in ASH neurons }\end{array}$ & Itzhak Mano \\
\hline $\begin{array}{l}\text { IMN57: xuEx929; eat-4 (ky5) III ; glt- } \\
\text { 3(bz34), glt-6(tm1316), glt-7(tm1641) IV } \\
\text { zfIS42 }\end{array}$ & $\begin{array}{l}\text { Loss of function in GLT-3, } \\
\text { GLT-6, GLT-7, and EAT-4, } \\
\text { expressing functional EAT- } \\
4 \text { cDNA specifically in ASH } \\
\text { neurons }\end{array}$ & Itzhak Mano \\
\hline $\begin{array}{l}\text { IMN59: xuEx929; glt-4 (bz69) X ; eat-4 } \\
\text { (ky5) III ; zfIS42 }\end{array}$ & $\begin{array}{l}\text { Loss of function in GLT-4 } \\
\text { and EAT-4, expressing } \\
\text { functional EAT-4 cDNA } \\
\text { specifically in ASH neurons }\end{array}$ & Itzhak Mano \\
\hline $\begin{array}{l}\text { IMN60: } P_{g c y-6:: e a t-4(c D N A) ; ~ e a t-4(k y 5)} \\
\text { III ; zfIS42 }\end{array}$ & $\begin{array}{l}\text { Loss of function in EAT-4, } \\
\text { expressing functional EAT- } \\
4 \text { cDNA specifically in } \\
\text { ASEL neurons }\end{array}$ & Itzhak Mano \\
\hline $\begin{array}{l}\text { IMN61: } P_{g c y-6:: e a t-4(c D N A)} ; \text { glt-1 (ok206) } \\
\text { X ; eat-4 (ky5) III ; zfIS42 }\end{array}$ & $\begin{array}{l}\text { Loss of function in GLT-1 } \\
\text { and EAT-4, expressing } \\
\text { functional EAT-4 cDNA } \\
\text { specifically in ASEL } \\
\text { neurons }\end{array}$ & Itzhak Mano \\
\hline $\begin{array}{l}\text { IMN62: } P_{\text {gcy-6}}:: e a t-4(c D N A) ; \text { eat-4 (ky5) } \\
\text { III ; glt-3(bz34), glt-6(tm1316), glt- } \\
\text { 7(tm1641) IV; zfIS42 }\end{array}$ & $\begin{array}{l}\text { Loss of function in GLT-3, } \\
\text { GLT-6, GLT-7, and EAT-4, } \\
\text { expressing functional EAT- } \\
4 \text { cDNA specifically in } \\
\text { ASEL neurons }\end{array}$ & Itzhak Mano \\
\hline $\begin{array}{l}\text { IMN63: } P_{\text {gcy-6-6:eat-4(cDNA) ; glt-4 (bz69) }} \\
\text { X ; eat-4 (ky5) III ; zfIS42 }\end{array}$ & $\begin{array}{l}\text { Loss of function in GLT-4 } \\
\text { and EAT-4, expressing } \\
\text { functional EAT-4 cDNA } \\
\text { specifically in ASEL } \\
\text { neurons }\end{array}$ & Itzhak Mano \\
\hline glt-3(bz34) IV; zfIs42 & $\begin{array}{l}\text { Loss of function in GLT-3, } \\
\text { expressing GCaMP3 in } \\
\text { AVA interneurons }\end{array}$ & Itzhak Mano \\
\hline $\begin{array}{l}\text { IMN64: } P_{g l t-3}: \text { glt-3cDNA:SL2:mCherry ; } \\
P_{\text {mec-7::H2B:GFP; glt-3(bz34) IV; zfIS42 }}\end{array}$ & $\begin{array}{l}\text { Loss of function in GLT-3, } \\
\text { expressing functional GLT- } \\
3 \text { cDNA under the } g l t-3 \\
\text { promoter }(15 \mathrm{ng} / \mathrm{uL}) \text {, and } \\
150 \mathrm{ng} / \mathrm{uL} \text { of } P_{\text {mec- }} \\
\text { 7:H2B:GFP coinjection } \\
\text { marker. }\end{array}$ & Itzhak Mano \\
\hline $\begin{array}{l}\text { IMN65: } P_{g l t-3}: g l t-1 c D N A: S L 2: m C h e r r y ; \\
P_{\text {mec-7:H2B:GFP; } \text {; }} \text {; }-3(b z 34) I V ; z f I S 42\end{array}$ & $\begin{array}{l}\text { Loss of function in GLT-3, } \\
\text { expressing functional GLT- } \\
1 \mathrm{cDNA} \text { under the } g l t-3 \\
\text { promoter }(15 \mathrm{ng} / \mathrm{uL}) \text {, and } \\
150 \mathrm{ng} / \mathrm{uL} \text { of } P \text { mec- } \\
\text { 7:H2B:GFP coinjection } \\
\text { marker. }\end{array}$ & Itzhak Mano \\
\hline
\end{tabular}




\begin{tabular}{|c|c|c|}
\hline 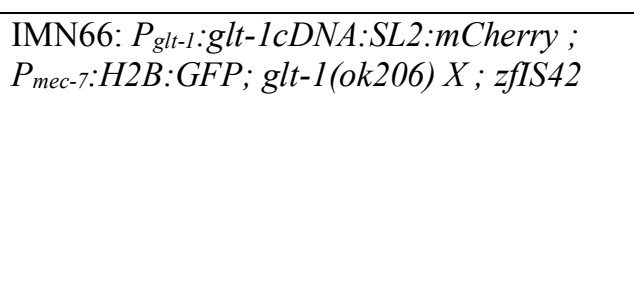 & $\begin{array}{l}\text { Loss of function in GLT-1, } \\
\text { expressing functional GLT- } \\
1 \text { cDNA under the glt- } 1 \\
\text { promoter }(50 \mathrm{ng} / \mathrm{uL}), \text { and } \\
150 \mathrm{ng} / \mathrm{uL} \text { of } P \text { mec- } \\
\text { 7:H2B:GFP coinjection } \\
\text { marker. }\end{array}$ & Itzhak Mano \\
\hline $\begin{array}{l}\text { IMN67: } P_{\text {glt-1 }}: \text { glt-3cDNA:SL2:mCherry; } \\
P_{\text {mec-7:H2B:GFP; glt-1(ok206) X; zfIS42 }}\end{array}$ & $\begin{array}{l}\text { Loss of function in GLT-1, } \\
\text { expressing functional GLT- } \\
3 \text { cDNA under the glt- } 1 \\
\text { promoter }(50 \mathrm{ng} / \mathrm{uL}), \text { and } \\
150 \mathrm{ng} / \mathrm{uL} \text { of } P_{\text {mec- }} \\
\text { 7:H2B:GFP coinjection } \\
\text { marker. }\end{array}$ & Itzhak Mano \\
\hline $\begin{array}{l}\text { DK5389: taEx171 [P } P_{n p r-9:}: G C a M P 6, P_{\text {lin }} \\
\text { 44::mCherry] }\end{array}$ & $\begin{array}{l}\text { GCaMP6 expressed in AIB } \\
\text { interneuron }\end{array}$ & Motomichi Doi ${ }^{19}$ \\
\hline taEx171;glt-1(ok206) X & $\begin{array}{l}\text { Loss of function in GLT-1, } \\
\text { GCaMP6 expressed in AIB } \\
\text { interneuron }\end{array}$ & Itzhak Mano \\
\hline taEx171;glt-4(bz34) X & $\begin{array}{l}\text { Loss of function in GLT-4, } \\
\text { GCaMP6 expressed in AIB } \\
\text { interneuron }\end{array}$ & Itzhak Mano \\
\hline $\begin{array}{l}\text { taEx171; glt-3(bz34), glt-6(tm1316), glt- } \\
\text { 7(tm1641) IV }\end{array}$ & $\begin{array}{l}\text { Loss of function in GLT-3, } \\
\text { GLT-6, and GLT-7, } \\
\text { GCaMP6 expressed in AIB } \\
\text { interneuron }\end{array}$ & Itzhak Mano \\
\hline $\begin{array}{l}\text { taEx171; } P_{\text {myo- } 2:: H i s C l 1:: S L 2:: m \text { Cherry; }} \\
P_{\text {myo-3: }: \text { HisCl1::SL2::mCherry }}\end{array}$ & $\begin{array}{l}\text { GCaMP6 expressed in AIB } \\
\text { interneuron, HisCl1 } \\
\text { expressed in pharyngeal and } \\
\text { body wall muscles (injected } \\
50 \mathrm{ng} / \mathrm{uL} \text { of each } \mathrm{HisCl} \\
\text { construct) }\end{array}$ & Itzhak Mano \\
\hline 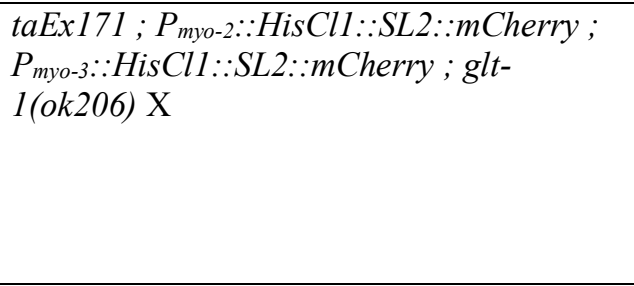 & $\begin{array}{l}\text { Loss of function in GLT-1, } \\
\text { GCaMP6 expressed in AIB } \\
\text { interneuron, HisCl1 } \\
\text { expressed in pharyngeal and } \\
\text { body wall muscles (injected } \\
50 \mathrm{ng} / \mathrm{uL} \text { of each HisCl } \\
\text { construct) }\end{array}$ & Itzhak Mano \\
\hline 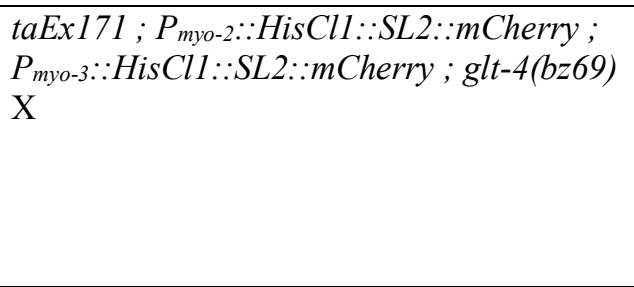 & $\begin{array}{l}\text { Loss of function in GLT-4, } \\
\text { GCaMP6 expressed in AIB } \\
\text { interneuron, HisCl1 } \\
\text { expressed in pharyngeal and } \\
\text { body wall muscles (injected } \\
50 \mathrm{ng} / \mathrm{uL} \text { of each HisCl } \\
\text { construct) }\end{array}$ & Itzhak Mano \\
\hline 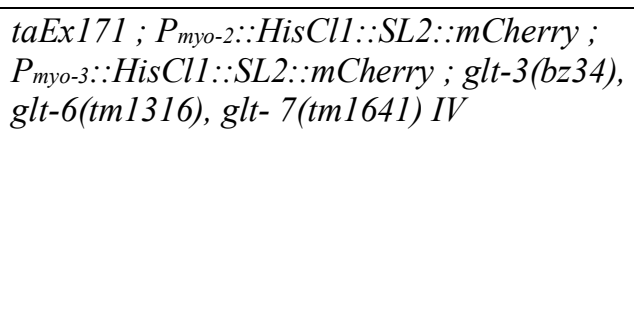 & $\begin{array}{l}\text { Loss of function in GLT-3, } \\
\text { GLT-6, and GLT-7, } \\
\text { GCaMP6 expressed in AIB } \\
\text { interneuron, HisCl1 } \\
\text { expressed in pharyngeal and } \\
\text { body wall muscles (injected } \\
50 \mathrm{ng} / \mathrm{uL} \text { of each HisCl } \\
\text { construct) }\end{array}$ & Itzhak Mano \\
\hline
\end{tabular}




\begin{tabular}{|c|c|c|}
\hline 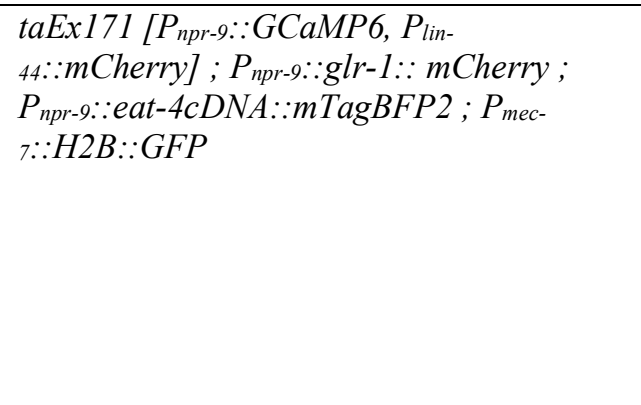 & $\begin{array}{l}\text { GCaMP6 expressed in AIB } \\
\text { interneuron, with proximal } \\
\text { AIB neurites labelled with } \\
\text { GLR-1::mCherry }(15 \mathrm{ng} / \mathrm{uL}) \\
\text { and distal AIB neurites } \\
\text { labelled with EAT- } \\
4:: \mathrm{mTagBFP} 2(15 \mathrm{ng} / \mathrm{uL}) . \\
150 \mathrm{ng} / \mathrm{uL} \text { of co-injection } \\
\text { marker } P_{\text {mec- }-:: H 2 B:: G F P} \\
\text { was used. }\end{array}$ & Itzhak Mano \\
\hline 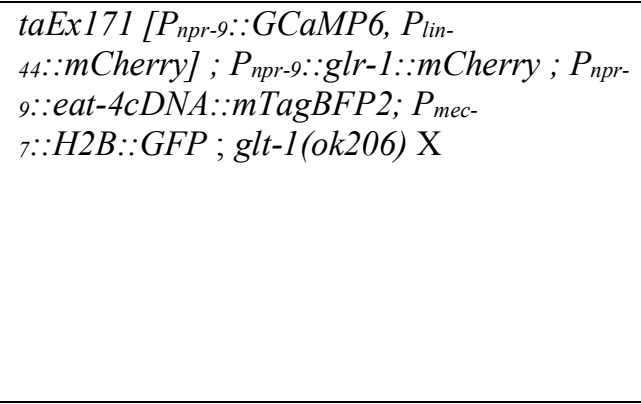 & $\begin{array}{l}\text { Loss of function in GLT-1, } \\
\text { GCaMP6 expressed in AIB } \\
\text { interneuron, proximal AIB } \\
\text { neurites labelled with GLR- } \\
1:: \text { mCherry }(15 \mathrm{ng} / \mathrm{uL}) \text { and } \\
\text { distal AIB neurites (15 } \\
\text { ng/uL) labelled with EAT- } \\
4:: \mathrm{mTagBFP} 2.150 \mathrm{ng} / \mathrm{uL} \text { of } \\
\text { co-injection marker } P \text { mec- } \\
7:: H 2 B:: G F P \text { was used. }\end{array}$ & Itzhak Mano \\
\hline 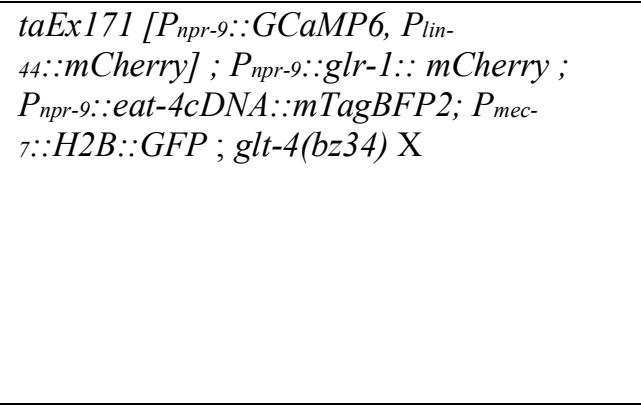 & $\begin{array}{l}\text { Loss of function in GLT-4, } \\
\text { GCaMP6 expressed in AIB } \\
\text { interneuron, proximal AIB } \\
\text { neurites labelled with GLR- } \\
1:: \text { mCherry }(15 \mathrm{ng} / \mathrm{uL}) \text { and } \\
\text { distal AIB neurites labelled } \\
\text { with EAT- } 4:: \mathrm{mTagBFP} 2 \\
(15 \mathrm{ng} / \mathrm{uL}) .150 \mathrm{ng} / \mathrm{uL} \text { of } \\
\text { co-injection marker } P_{\text {mec- }} \\
7:: H 2 B:: G F P \text { was used. } \\
\end{array}$ & Itzhak Mano \\
\hline 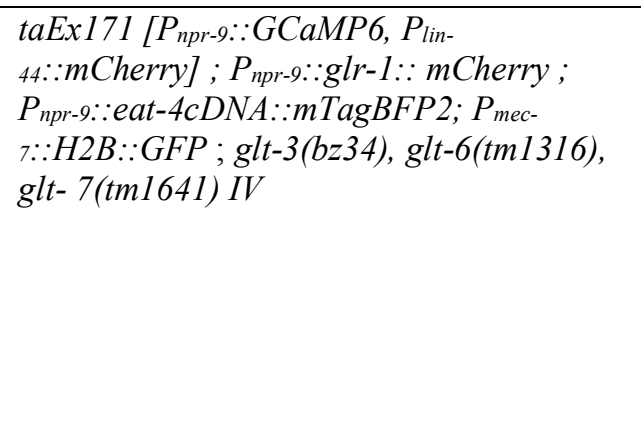 & $\begin{array}{l}\text { Loss of function in GLT-3, } \\
\text { GLT-6, and GLT-7, } \\
\text { GCaMP6 expressed in AIB } \\
\text { interneuron, proximal AIB } \\
\text { neurites labelled with GLR- } \\
1:: \text { mCherry }(15 \mathrm{ng} / \mathrm{uL}) \text { and } \\
\text { distal AIB neurites labelled } \\
\text { with EAT-4::mTagBFP2 } \\
(15 \mathrm{ng} / \mathrm{uL}) .150 \mathrm{ng} / \mathrm{uL} \text { of co- } \\
\text { injection marker } P_{\text {mec- }} \\
7: \because H 2 B:: G F P \text { was used. }\end{array}$ & Itzhak Mano \\
\hline $\begin{array}{l}\text { otIs669 V; } P_{\text {glt-4: }:: S f G F P ;} P_{m y o-} \\
\text { з::HisCl1 ::SL2::mCherry }\end{array}$ & $\begin{array}{l}\text { NeuroPAL strain with } \\
\text { reporters integrated into } \\
\text { chromosome V. injected } \\
\text { with } 20 \mathrm{ng} / \mathrm{uL} \text { of Pglt- } \\
4:: \mathrm{sfGFP}, \text { and with } 45 \mathrm{ng} / \mathrm{uL} \\
\text { of } P_{\text {myo-3 }: \text { HisCl::mCherry }} \\
\text { as co-injection marker. }\end{array}$ & Itzhak Mano \\
\hline
\end{tabular}




\subsection{References}

1. Berger, A. J., Hart, A. C. \& Kaplan, J. M. G $\alpha$ s-Induced Neurodegeneration in

Caenorhabditis elegans. The Journal of Neuroscience 18, 2871-2880 (1998).

2. Mano, I. \& Driscoll, M. Caenorhabditis elegans glutamate transporter deletion induces

AMPA-receptor/adenylyl cyclase 9-dependent excitotoxicity. Journal of Neurochemistry 108, 1373-1384 (2009).

3. Feldmann, K. G. et al. Non-canonical activation of CREB mediates neuroprotection in a Caenorhabditis elegans model of excitotoxic necrosis. J. Neurochem. 148, 531-549 (2019).

4. Zou, W. et al. Decoding the intensity of sensory input by two glutamate receptors in one C. elegans interneuron. Nature Communications 9, (2018).

5. Gordus, A., Pokala, N., Levy, S., Flavell, S. W. \& Bargmann, C. I. Feedback from Network States Generates Variability in a Probabilistic Olfactory Circuit. Cell 161, 215-227 (2015).

6. Arriza, J. L., Eliasof, S., Kavanaugh, M. P. \& Amara, S. G. Excitatory amino acid transporter 5, a retinal glutamate transporter coupled to a chloride conductance. Proceedings of the National Academy of Sciences 94, 4155-4160 (1997).

7. Danbolt, N. C. Glutamate uptake. Progress in Neurobiology 65, 1-105 (2001).

8. Wersinger, E. et al. The glutamate transporter EAAT5 works as a presynaptic receptor in mouse rod bipolar cells: Presynaptic inhibition by glutamate transporters. The Journal of Physiology 577, 221-234 (2006).

9. Tzingounis, A. V. \& Wadiche, J. I. Glutamate transporters: confining runaway excitation by shaping synaptic transmission. Nature Reviews Neuroscience 8, 935-947 (2007). 
10. Veruki, M. L., Mørkve, S. H. \& Hartveit, E. Activation of a presynaptic glutamate transporter regulates synaptic transmission through electrical signaling. Nat Neurosci 9, 13881396 (2006).

11. Chen, I. et al. Glutamate transporters have a chloride channel with two hydrophobic gates. Nature 591, 327-331 (2021).

12. Ventimiglia, D. \& Bargmann, C. I. Diverse modes of synaptic signaling, regulation, and plasticity distinguish two classes of C. elegans glutamatergic neurons. eLife 6, e31234 (2017). 13. Hardaway, J. A. et al. Glial Expression of the Caenorhabditis elegans Gene swip-10 Supports Glutamate Dependent Control of Extrasynaptic Dopamine Signaling. Journal of Neuroscience 35, 9409-9423 (2015).

14. Cook, S. J. et al. Whole-animal connectomes of both Caenorhabditis elegans sexes. Nature 571, 63-71 (2019).

15. Choi, M.-K., Liu, H., Wu, T., Yang, W. \& Zhang, Y. NMDAR-mediated modulation of gap junction circuit regulates olfactory learning in C. elegans. Nat Commun 11, 3467 (2020). 16. Ryan, R. M., Mitrovic, A. D. \& Vandenberg, R. J. The Chloride Permeation Pathway of a Glutamate Transporter and Its Proximity to the Glutamate Translocation Pathway. Journal of Biological Chemistry 279, 20742-20751 (2004).

17. Verdon, G. \& Boudker, O. Crystal structure of an asymmetric trimer of a bacterial glutamate transporter homolog. Nat Struct Mol Biol 19, 355-357 (2012).

18. Frøkjær-Jensen, C. et al. Single-copy insertion of transgenes in Caenorhabditis elegans. Nat Genet 40, 1375-1383 (2008).

19. Frøkjær-Jensen, C., Davis, M. W., Ailion, M. \& Jorgensen, E. M. Improved Mos1mediated transgenesis in C. elegans. Nat Methods 9, 117-118 (2012). 
20. Frøkjær-Jensen, C. et al. Random and targeted transgene insertion in Caenorhabditis elegans using a modified Mos1 transposon. Nat Methods 11, 529-534 (2014).

21. Schrödinger, L. L. C. The PyMOL Molecular Graphics System. (2018).

22. Yernool, D., Boudker, O., Jin, Y.\& Gouaux, E. Structure of a glutamate transporter homologue from Pyrococcus horikoshii. Nature 431, 811-818 (2004).

23. Boudker, O., Ryan, R. M., Yernool, D., Shimamoto, K. \& Gouaux, E. Coupling substrate and ion binding to extracellular gate of a sodium-dependent aspartate transporter. Nature $\mathbf{4 4 5}$, 387-393 (2007).

24. Bastug, T. et al. Position of the Third Na+ Site in the Aspartate Transporter GltPh and the Human Glutamate Transporter, EAAT1. PLoS ONE 7, e33058 (2012).

25. Kuramochi, M. \& Doi, M. An Excitatory/Inhibitory Switch From Asymmetric Sensory Neurons Defines Postsynaptic Tuning for a Rapid Response to $\mathrm{NaCl}$ in Caenorhabditis elegans. Frontiers in Molecular Neuroscience 11, (2019).

26. Li, Z. et al. A C. elegans neuron both promotes and suppresses motor behavior to fine tune motor output. 36 .

27. Marvin, J. S. et al. An optimized fluorescent probe for visualizing glutamate neurotransmission. Nature methods 10, 162-170 (2013).

28. Li, N. \& van der Kooy, D. Mutations in the guanylate cyclase gcy-28 neuronally dissociate naïve attraction and memory retrieval. European Journal of Neuroscience 48, 33673378 (2018).

29. Mano, I., Straud, S. \& Driscoll, M. Caenorhabditis elegans glutamate transporters influence synaptic function and behavior at sites distant from the synapse. Journal of Biological Chemistry 282, 34412-34419 (2007). 
30. Pédelacq, J.-D., Cabantous, S., Tran, T., Terwilliger, T. C. \& Waldo, G. S. Engineering and characterization of a superfolder green fluorescent protein. Nature Biotechnology 24, 79-88 (2006).

31. Yemini, E. et al. NeuroPAL: A Multicolor Atlas for Whole-Brain Neuronal Identification in C. elegans. Cell 184, 272-288.e11 (2021).

32. Cao, J. et al. Comprehensive single-cell transcriptional profiling of a multicellular organism. Science 357, 661-667 (2017).

33. Jin, X., Pokala, N. \& Bargmann, C. I. Distinct Circuits for the Formation and Retrieval of an Imprinted Olfactory Memory. Cell 164, 632-643 (2016).

34. Hammarlund, M., Hobert, O., Miller, D. M. \& Sestan, N. The CeNGEN Project: The Complete Gene Expression Map of an Entire Nervous System. Neuron 99, 430-433 (2018).

35. Kato, S., Xu, Y., Cho, C. E., Abbott, L. F. \& Bargmann, C. I. Temporal Responses of $C$. elegans Chemosensory Neurons Are Preserved in Behavioral Dynamics. Neuron 81, 616-628 (2014).

36. Gene. National Center for Biotechnology Information https://www.ncbi.nlm.nih.gov/ (1988).

37. Altschu, S. F., Gish, W., Miller, W., Myers, E. W. \& Lipman, D. J. Basic Local Alignment Search Tool. J Mol Bio 215, 403-410 (1990).

38. Zhang, Y. I-TASSER server for protein 3D structure prediction. BioMed Central Bioinformatics 9, 1-8 (2008).

39. Ambrish, R., Kucukural, A. \& Zhang, Y. I-TASSER: a unified platform for automated protein structure and function prediction. Nature Protocols 5, 725-738 (2010). 
40. Zhang, Y. The I-TASSER Suite: protein structure and function prediction. Nature Methods 12, 1-8 (2015). 